\title{
The Role of Rivet Installation on the Fatigue Performance of Riveted Lap Joints
}

by

\author{
Calvin D. Rans \\ B.Eng, M.A.Sc.
}

A Dissertation submitted to the Faculty of Graduate Studies and Research

in partial fulfilment of

the requirements for the degree of

Doctor of Philosophy

\author{
Ottawa-Carleton Institute for \\ Mechanical and Aerospace Engineering \\ Department of Mechanical and Aerospace Engineering \\ Carleton University \\ Ottawa, Ontario, Canada
}

August 2007

Copyright (C)

August 2007 


$\begin{array}{ll}\begin{array}{l}\text { Library and } \\ \text { Archives Canada }\end{array} & \begin{array}{l}\text { Bibliothèque et } \\ \text { Archives Canada }\end{array} \\ \begin{array}{l}\text { Published Heritage } \\ \text { Branch }\end{array} & \begin{array}{l}\text { Direction du } \\ \text { Patrimoine de l'édition }\end{array} \\ \begin{array}{l}\text { 395 Wellington Street } \\ \text { Ottawa ON K1A ON4 }\end{array} & \begin{array}{l}\text { 395, rue Wellington } \\ \text { Ottawa ON K1A ON4 } \\ \text { Canada }\end{array}\end{array}$

Your file Votre référence ISBN: 978-0-494-33509-3 Our file Notre référence ISBN: 978-0-494-33509-3

NOTICE:

The author has granted a nonexclusive license allowing Library and Archives Canada to reproduce, publish, archive, preserve, conserve, communicate to the public by telecommunication or on the Internet, loan, distribute and sell theses worldwide, for commercial or noncommercial purposes, in microform, paper, electronic and/or any other formats.

The author retains copyright ownership and moral rights in this thesis. Neither the thesis nor substantial extracts from it may be printed or otherwise reproduced without the author's permission.
AVIS:

L'auteur a accordé une licence non exclusive permettant à la Bibliothèque et Archives Canada de reproduire, publier, archiver, sauvegarder, conserver, transmettre au public par télécommunication ou par l'Internet, prêter, distribuer et vendre des thèses partout dans le monde, à des fins commerciales ou autres, sur support microforme, papier, électronique et/ou autres formats.

L'auteur conserve la propriété du droit d'auteur et des droits moraux qui protège cette thèse. $\mathrm{Ni}$ la thèse ni des extraits substantiels de celle-ci ne doivent être imprimés ou autrement reproduits sans son autorisation.
In compliance with the Canadian

Privacy Act some supporting forms may have been removed from this thesis.

While these forms may be included in the document page count, their removal does not represent any loss of content from the thesis.
Conformément à la loi canadienne sur la protection de la vie privée, quelques formulaires secondaires ont été enlevés de cette thèse.

Bien que ces formulaires aient inclus dans la pagination, il n'y aura aucun contenu manquant.

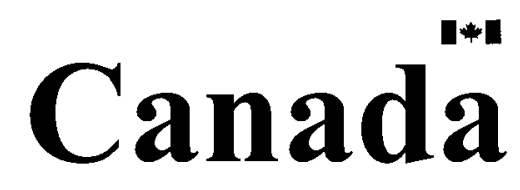




\section{Abstract}

Solid rivets are widely used as mechanical fasteners in airframe applications due to their relative low cost and good fatigue performance. Although rivet installation is known to influence this fatigue performance, variabilities in hand riveting practices make exploiting rivet installation as a design variable difficult. Developments in riveting technology have led to force-controlled rivet squeezers and fully automated riveting gantries, improving the consistency of rivet installation and providing the opportunity to exploit its influence on fatigue.

This dissertation describes a research program undertaken to examine the influence of rivet installation on the fatigue performance of riveted lap joints and identify what aspects can be exploited during design. A combination of finite element analyses and experimental techniques were used to investigate the role of rivet installation on the formation of residual stresses and on secondary bending stresses in a loaded joint, two aspects established as critical to the fatigue performance of riveted lap joints. Crack growth reconstructions of fracture surfaces marked using a special marker fatigue spectrum were also completed in order to quantify the effects of these residual and secondary bending stresses on fatigue performance. Additionally, variations in the effects of rivet installation on traditional monolithic aluminum sheet materials and hybrid aluminum-fibre glass laminates known as GLARE were also investigated.

Results from these investigations provided new insights into the role of rivet installation on fatigue. The radial expansion mechanism to which residual stress formation during riveting is typically attributed was observed to be a secondary mechanism relative to the through-thickness compression of the joined sheets. The location and magnitude of peak 
secondary bending stresses were found to be directly influenced by rivet head geometry. In certain cases, shifts in the location of peak secondary bending stress were found to negate the benefits of residual stresses formed during riveting. Finally, differences in the influences of rivet installation on GLARE and monolithic aluminum sheet were also identified. The presence of the fibre layer in GLARE alters the residual and secondary bending stress states, indicating a potential difference in joint designs optimized for aluminum and GLARE. 
I dedicate this work to the loving memory of my Grandfather, George Henry MacNab. Thanks for the quarter Grandpa. It was more valuable a gift than you could ever imagine. 


\section{Acknowledgments}

Although my name appears on this dissertation as the sole author and contributor to its contents, in reality, it is an achievement made possible only by the efforts of many. I would like to express my sincerest gratitude to all those who supported me through the completion of my research.

First of all, I would like to acknowledge my supervisor Prof. Paul Straznicky. You have taught me so very much, including how to properly use a semicolon, that Czech beer really does taste better than Canadian beer, and that experimental testing always takes five times longer than expected. In all seriousness, I would not have been able to complete such a milestone in my academic career without your guidance and support. I would also like to acknowledge my co-supervisor René Alderliesten from the Technical University of Delft. Although my time in Delft was short, it was worthwhile and valuable, both academically and personally.

At the heart of any successful research program are the support staff. This research program was no exception. First of all, I would like to thank Gery Clements, Fred Barrett, and Steve Truttmann for their help in keeping the structures laboratory running and my experimental testing on schedule. Secondly, Alex Proctor and the MAE workshop for their help in manufacturing testing equipment and specimens. Thirdly, Lewis Ling and Peter Jones for their patience and guidance during my many hours in the SEM laboratory. Finally, Marlene Groves, Christie Egbert, and Nancy Powell for guiding me through all the administrative hiccups during my $\mathrm{PhD}$ and my entire 9 years at Carleton. I owe a great debt of gratitude to each and every one of you. 
In addition to the support staff, there were several groups of people who contributed greatly to my research. First, I would like to thank Gewana Abdelmesih and Caroline Li for their help during the fatigue testing phase of my research. Without their help I would not have been able to focus on other problems in my research. Second, I would like to thank George Shi and Gang Li from the National Research Council of Canada for their consultations regarding my finite element studies and for access to their invaluable neutron diffraction data. Also from the National Research Council of Canada, I would like to thank David Backman for his help in performing DIC experiments as part of my finite element verification efforts.

My 6 years of graduate studies at Carleton would not have been the same without the support and friendship of the many graduate students within the department. I will always remember the $3284 \mathrm{ME}$ crew and all the good time we had together.

Finally, I would like to thank my parents, Richard and Jean Rans, and the rest of my family for their love and support through my entire PhD. I love you guys and I couldn't have done this without you.

If we knew what it was we were doing, it would not be called research, would it?

- Albert Einstein 


\section{Table of Contents}

Abstract $\quad$ iii

Acknowledgments $\quad$ vi

Table of Contents viii

List of Tables $\quad$ xii

List of Figures $\quad$ xiii

$\begin{array}{ll}\text { Nomenclature } & \text { xix }\end{array}$

1 Introduction 1

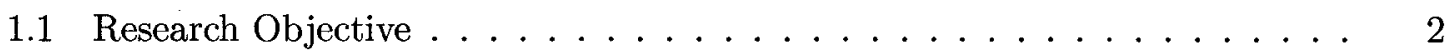

1.2 Dissertation Overview . . . . . . . . . . . . . . . 4

2 Background 5

2.1 Introduction . . . . . . . . . . . . . . . . 5

2.2 Solid Rivets . . . . . . . . . . . . . . . . . . . 6

2.2 .1 Rivet Installation . . . . . . . . . . . . . . 6

2.2 .2 Rivet Type and Geometry . . . . . . . . . . . . . 9

2.3 Lap Joint Nomenclature . . . . . . . . . . . . . . . . . . 11

2.4 Local Stress Field in a Loaded Lap Splice . . . . . . . . . . . . . . 12

2.4 .1 Load Transfer. . . . . . . . . . . . . . . . . 13

2.4 .2 Secondary Bending . . . . . . . . . . . . . . . 15

viii 
2.4 .3 Rivet Interference $\ldots \ldots \ldots \ldots \ldots$. . . . . . . . . 17

2.5 GLARE Laminates . . . . . . . . . . . . . . . . . . 20

2.5.1 GLARE Variants . . . . . . . . . . . . . . 20

2.5.2 Fatigue Behaviour ......................... 22

3 Cold Expansion Induced Residual Stresses $\quad 24$

3.1 Introduction . . . . . . . . . . . . . . . . . 24

3.2 Review of Cold Expansion . . . . . . . . . . . . . . . 25

3.3 Cold Expansion Finite Element Model . . . . . . . . . . . . . . . . 27

3.3 .1 Model Description . . . . . . . . . . . . . . . 27

3.3 .2 Material Modelling . . . . . . . . . . . . . 30

3.3.3 Delamination Modelling for GLARE . . . . . . . . . . . 30

3.3 .4 Model Verification . . . . . . . . . . . . . . . 32

3.4 Finite Element Results . . . . . . . . . . . . . . . . . . . . 33

3.4.1 2024-T3 Residual Stress Distribution . . . . . . . . . . . 33

3.4.2 GLARE3-2/1-0.3 Residual Stress Distribution . . . . . . . . . 35

3.4.3 Influence of Delamination Model . . . . . . . . . . . . 38

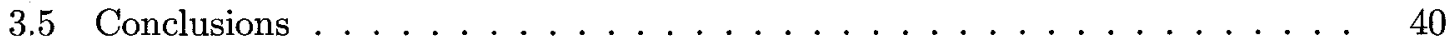

4 Riveting Induced Residual Stresses $\quad 42$

4.1 Introduction . . . . . . . . . . . . . . . . . 42

4.2 Review of Riveting Induced Residual Stresses . . . . . . . . . . . . 43

4.3 Rivet Installation Finite Element Model . . . . . . . . . . . . . . . . 45

4.3 .1 Model Description . . . . . . . . . . . . . . 45

4.3 .2 Rivet Geometries . . . . . . . . . . . . . . . 47

4.3 .3 Material Modelling . . . . . . . . . . . . . . . . . . 48

4.3.4 Delamination Modelling for GLARE . . . . . . . . . . . . 49

4.3.5 Model Verification . . . . . . . . . . . . . . . 51

4.4 Finite Element Results . . . . . . . . . . . . . . . . . . 53

4.4 .1 Hole Expansion . . . . . . . . . . . . . . . 53

$\mathrm{ix}$ 
4.4.2 Generalized Residual Stress Distribution . . . . . . . . . . . 55

4.4 .3 Effect of Countersink Depth . . . . . . . . . . . . . 57

4.4.4 Faying Surface Residual Stresses . . . . . . . . . . . . . 60

4.4 .5 Influence of Sheet Material $\ldots \ldots \ldots 62$

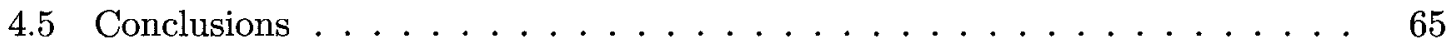

5 Secondary Bending and Splice Loading $\quad 67$

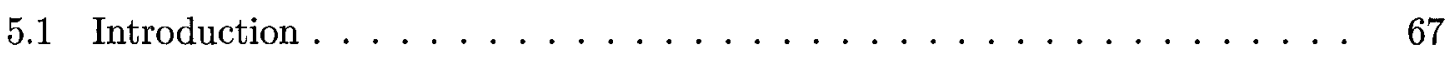

5.2 Review of Secondary Bending . . . . . . . . . . . . . . 67

5.3 Splice Loading Finite Element Model . . . . . . . . . . . . . . . . 69

5.3 .1 Model Description . . . . . . . . . . . . . . 69

5.3 .2 Model Verification . . . . . . . . . . . . . . . 71

5.3.3 Secondary Bending Calculation . . . . . . . . . . . 73

5.4 Finite Element Results . . . . . . . . . . . . . . . . 76

5.4.1 Neutral Line Model Comparison . . . . . . . . . . . . 77

5.4 .2 Peak Secondary Bending . . . . . . . . . . . . 80

5.4 .3 Influence of Sheet Material $\ldots \ldots \ldots 83$

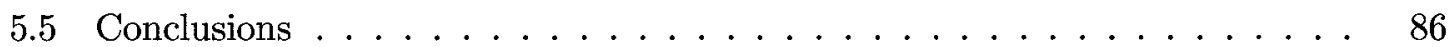

6 Crack Growth $\quad 88$

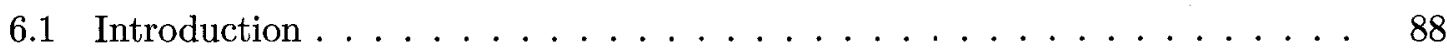

6.2 Review of Crack Growth in Riveted Splices . . . . . . . . . . . 88

6.3 Fatigue Crack Growth Tests . . . . . . . . . . . . . . . . . 91

6.3 .1 Test Specimen Geometry _. . . . . . . . . . . . . 92

6.3 .2 Fatigue Test Spectrum . . . . . . . . . . . . . . . . . 93

6.3 .3 Test Matrix . . . . . . . . . . . . . . 95

6.3.4 Crack Growth Reconstruction . . . . . . . . . . . . 95

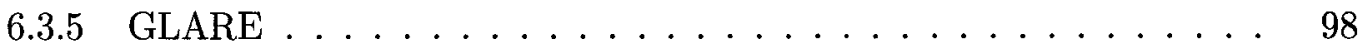

6.4 Overall Fatigue Life Results . . . . . . . . . . . . . . . . . . . 99

6.5 Crack Growth Reconstruction Results . . . . . . . . . . . . . . 103 
6.5.1 General SEM Observations . . . . . . . . . . . . . 103

6.5.2 Countersunk Rivet Joint Reconstructions _ . . . . . . . . . . . . 105

6.5.3 Universal Rivet Joint Reconstructions . . . . . . . . . . . . . . 109

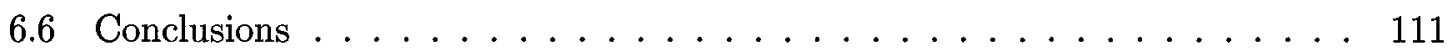

$\begin{array}{lll}7 & \text { Discussion } & 113\end{array}$

7.1 Formation of Residual Stresses . . . . . . . . . . . . . . . . . . . . . 114

7.2 Secondary Bending . . . . . . . . . . . . . . . 115

7.3 Fatigue Performance . . . . . . . . . . . . . . . 116

7.4 GLARE Laminates . . . . . . . . . . . . . . . . . . . . . 117

8 Conclusions and Statement of Contribution $\quad 120$

8.1 Conclusions . . . . . . . . . . . . . . . . . 121

8.2 Statement of Contribution . . . . . . . . . . . . . . . . 125

$\begin{array}{ll}\text { List of References } & 127\end{array}$

List of Publications $\quad 132$

Appendix A Finite Element Model Refinement 133

Appendix B Neutron Diffraction Verification Study 143

Appendix C Rivet Installation Finite Element Data $\quad 149$

$\begin{array}{ll}\text { Appendix D Splice Loading Finite Element Data } & 160\end{array}$

Appendix E Overall Fatigue Life Specimen Data $\quad 169$

$\begin{array}{lll}\text { Appendix F SEM Crack Growth Reconstruction Data } & 176\end{array}$ 


\section{List of Tables}

2.1 Rivet aluminum alloy designations . . . . . . . . . . . . . 11

2.2 Standard grades of GLARE . . . . . . . . . . . . . . . . 21

3.1 Cold expanded plate mesh details . . . . . . . . . . . . . . . . . . . 29

3.2 Summary of cold expansion FE material models . . . . . . . . . . . . 31

4.1 Summary of rivet installation FE material models $\ldots \ldots \ldots \ldots$

6.1 Test matrix for $10-4-6$ marker band fatigue tests $\ldots \ldots \ldots$. . . . . . 96

6.2 Nucleation period obtained from SEM reconstructions . . . . . . . . . 109

A.1 Convergence study mesh details . . . . . . . . . . . . . . . 135

A.2 Resultant FE mesh size and computation times . . . . . . . . . . . 142

E.1 Type 1 coupon fatigue life results $\ldots \ldots \ldots \ldots$

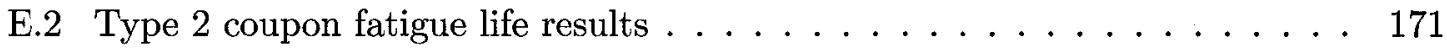

E.3 Universal rivet specimen confidence intervals . . . . . . . . . . . . 174

E.4 Countersunk rivet specimen confidence intervals . . . . . . . . . . . 175

F.1 Crack growth reconstruction data for CSK2.0-T1-SEM-01 rivet A2 $\ldots \ldots 178$

F.2 Crack growth reconstruction data for CSK2.0-T1-SEM-02 rivet B3 . . . 179

F.3 Crack growth reconstruction data for CSK2.0-T1-SEM-03 rivet B1 . . . 180

F.4 Crack growth reconstruction data for CSK3.0-T1-SEM-01 rivet B3 . . . . 181

F.5 Crack growth reconstruction data for CSK3.0-T1-SEM-02 rivet B2 . . . 182

F.6 Crack growth reconstruction data for CSK3.0-T1-SEM-03 rivet A1 . . . 183 


\section{List of Figures}

2.1 Types of tension loaded riveted joints $\ldots \ldots \ldots \ldots$

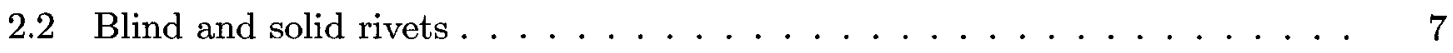

2.3 Rivet installation nomenclature . . . . . . . . . . . . 7

2.4 Stages of rivet squeezing $\ldots \ldots \ldots \ldots \ldots$

2.5 Types of solid rivets $\ldots \ldots \ldots \ldots \ldots$

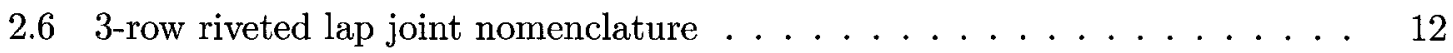

2.7 Illustration of local stress field components in a riveted lap joint . . . . . 13

2.8 Illustration of load carried by inner and outer sheets in a 3-row lap joint . . 14

2.9 Neutral line representation of a three-row riveted lap joint $\ldots \ldots \ldots$

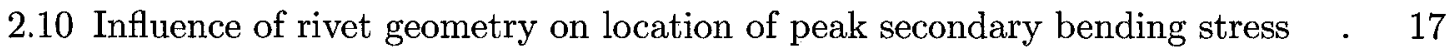

2.11 Residual stress in an infinite elastic-perfectly-plastic plate expanded by pres-

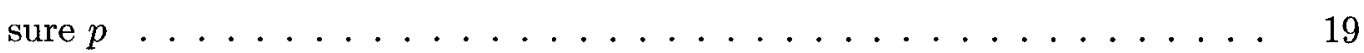

2.12 Typical fibre metal laminate layup . . . . . . . . . . . . 20

2.13 Fibre bridging mechanism in GLARE $\ldots \ldots \ldots \ldots \ldots . \ldots \ldots$

3.1 Schematic diagrams of split-mandrel and split-sleeve cold expansion processes 25

3.2 Illustration of key pattern for split-mandrel and split-sleeve cold expansion

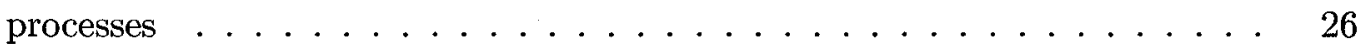

3.3 Schematic of cold expansion finite element model . . . . . . . . . . 28

3.4 Finite element plate mesh . . . . . . . . . . . . . . 28

3.5 Illustration of contact interface definition between GLARE layers . . . . . . 32

3.6 FE residual radial stress distribution for cold expanded 2024-T3 plate . . . 34

3.7 FE residual tangential stress distribution for cold expanded 2024-T3 plate . 34

xiii 
3.8 Comparison of 2024-T3 FE mid-plane residual stress results to analytical models . . . . . . . . . . . . . . . . . . .

3.9 FE mid-plane residual stress distributions for cold expanded GLARE3-2/1-

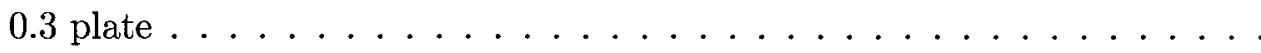

3.10 FE residual radial stress contours in GLARE for different delamination models 36

3.11 FE residual tangential stress contours in GLARE for different delamination models ............................... 36

3.12 Schematic of 2024-T3 and GLARE stress-strain curves . . . . . . . . . . 37

3.13 Residual tangential stress variation through plate thickness at hole edge . . 38

3.14 Effect of fibre layer anisotropy on mid-plane residual stress distributions in

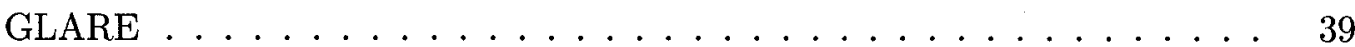

4.1 Schematic of rivet installation finite element model . . . . . . . . . . . . 45

4.2 Typical mesh density shown for deformed universal rivet in 2024-T3 plate • $\quad 46$

4.32 -D schematic of FE boundary conditions . . . . . . . . . . . . 47

4.4 Comparison of FE model and actual rivet geometries . . . . . . . . . . . 48

4.5 Comparison of 2117-T4 rivet material model true stress-strain curves . . . . 49

4.6 Comparison of FE residual strains to neutron diffraction data . . . . . . . 52

4.7 Comparison of FE and experimental bucking bar force-deflection curves . . 53

4.8 Radial expansion of rivet holes for universal and countersunk rivets at various

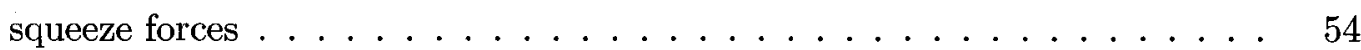

4.9 Schematic of typical residual tangential and radial stress distributions $\ldots \quad$. 56

4.10 Outer sheet mid-plane stresses under maximum applied squeeze force . . . . 56

4.11 Comparison of cold expansion analytical model and riveting FE model results of plastic zone radius vs. hole expansion . . . . . . . . . . . 57

4.12 Illustration of wedge expansion mechanism $\ldots \ldots \ldots \ldots$

4.13 Influence of countersink depth on outer sheet faying surface residual stress . $\quad 59$

4.14 Faying surface residual tangential stress distribution in 2024-T3 sheet for various rivet squeeze forces $\ldots \ldots \ldots \ldots$. . . . . . . . . . . 61

xiv 
4.15 Faying surface residual tangential stress distribution in GLARE3-2/1-0.3 sheet for various rivet squeeze forces $\ldots \ldots \ldots \ldots$

4.16 Comparison of residual rivet clamping force in 2024-T3 and GLARE3-2/1-0.3

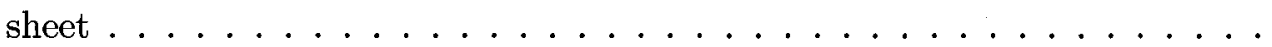

4.17 Angular variation of residual tangential stress distribution in GLARE3-2/10.3 with a universal rivet $\ldots \ldots \ldots \ldots \ldots \ldots$

5.1 Schematic of splice loading finite element model . . . . . . . . . . 70

5.2 Test setup for DIC verification test $\ldots \ldots \ldots \ldots \ldots \ldots$

5.3 Comparison of FE outer sheet surface strain results for a loaded lap joint to DIC experimental results $\ldots \ldots \ldots \ldots \ldots \ldots$

5.4 Application of superposition to resultant stress state in a splice under bending and membrane stresses $\ldots \ldots \ldots \ldots \ldots \ldots \ldots$

5.5 Resultant non-linear stress state in a splice when non-linear residual stress state is added using superposition . . . . . . . . . . . . .

5.6 Outer sheet through-thickness stress distribution in a 2024-T3 universal splice $2.5 \mathrm{~mm}$ above the critical rivet row $\ldots \ldots \ldots \ldots \ldots$

5.7 Outer sheet through-thickness stress distribution in a 2024-T3 countersunk splice along the net section of the critical rivet row $\ldots \ldots \ldots$ 75

5.8 Illustration of coordinate systems used for presentation of FE results $\ldots$. . $\quad 77$

$5.9 \mathrm{FE} K_{b}$ contour results within the outer sheet of a universal rivet joint $\ldots$. . 77

$5.10 \mathrm{FE} K_{b}$ contour results within the outer sheet of a countersunk rivet joint $\quad . \quad 78$

5.11 Comparison of $\mathrm{FE}$ outer sheet $K_{b}$ results to neutral line model predictions . $\quad 79$

5.12 Comparison of $\mathrm{FE}$ inner sheet $K_{b}$ results to neutral line model predictions .

5.13 Influence of $F_{S q}$ on the location and magnitude of maximum $K_{b}$ in a universal rivet joint

5.14 Influence of $F_{S q}$ on the location and magnitude of maximum $K_{b}$ in a countersunk rivet joint $\ldots \ldots \ldots \ldots \ldots \ldots \ldots \ldots \ldots \ldots \ldots$

5.15 Comparison of location and magnitude of maximum $K_{b}$ in universal and countersunk rivet joints $\ldots \ldots \ldots \ldots \ldots \ldots \ldots \ldots \ldots \ldots \ldots \ldots \ldots$ 
5.16 Comparison of FE $K_{b}$ results for GLARE3-2/1-0.3 sheet to neutral line model predictions $\ldots \ldots \ldots \ldots \ldots \ldots \ldots \ldots \ldots \ldots \ldots \ldots \ldots \ldots \ldots \ldots \ldots$

5.17 Comparison of location and magnitude of maximum $K_{b}$ in universal and countersunk GLARE3-2/1-0.3 rivet joints . . . . . . . . . 85

6.1 Schematic of $10-6-4$ marker-band spectrum $\ldots \ldots \ldots$. . . . . . . . . 91

6.2 Fatigue specimen geometry . . . . . . . . . . . . . . . 92

6.3 Schematic of modified 10-6-4 marker-band spectrum . . . . . . . . . . 93

6.4 Comparison of fatigue lives for coupons tested with and without the marker

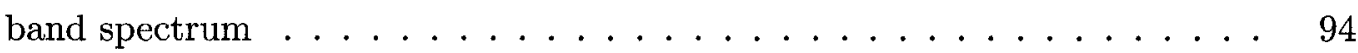

6.5 Examples of SEM reconstruction features $\ldots \ldots \ldots \ldots$. . . . . . . 97

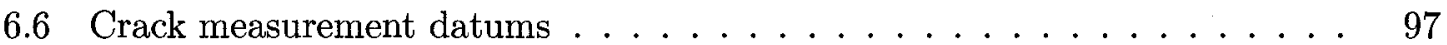

6.7 Overall fatigue lives for inner and outer sheet failure . . . . . . . . . . . 99

6.8 Comparison of overall fatigue lives for universal and countersunk joints (outer

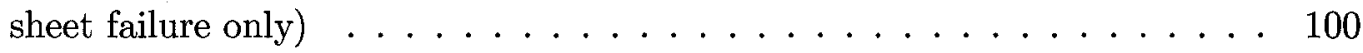

6.9 Location of fatigue damage in universal rivet joint fatigue specimens . . . 101

6.10 Location of fatigue damage in countersunk rivet joint fatigue specimens . . 102

6.11 Effects of Alclad layer on SEM reconstructions . . . . . . . . . . . . . 104

6.12 Fracture surface features which inhibited SEM reconstruction . . . . . . . 104

6.13 Typical crack front reconstruction for a countersunk rivet joint with $F_{S q}=$

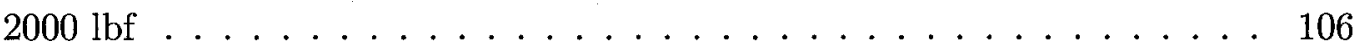

6.14 Typical crack front reconstruction for a countersunk rivet joint with $F_{S q}=$

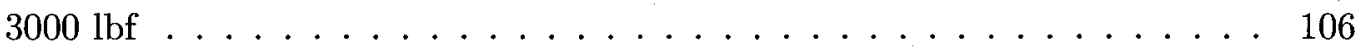

6.15 Crack growth behaviour for a countersunk rivet joint determined through SEM reconstructions . . . . . . . . . . . . . . . . . 106

6.16 Crack front reconstruction for U2.0-T1-SEM-01 . . . . . . . . . . . . . . 110

6.17 Crack front reconstruction for U3.0-T1-SEM-01 . . . . . . . . . . . . 110

A.1 Sample mesh convergence study results for cold expansion FE analysis . . . 136

A.2 Sample mesh convergence study results for rivet installation FE analysis . . 137

A.3 Sample mesh convergence study results for splice loading FE analysis . . . . 137

xvi 
A.4 Sample energy plots used to monitor kinetic and hourglass energy levels . . 140

A.5 Sample time scaling sensitivity results f . . . . . . . . . . . . . 141

B.1 Schematic of modified rivet installation finite element model . . . . . . . . . 144

B.2 Comparison of outer sheet residual radial strains f . . . . . . . . . . 146

B.3 Comparison of inner sheet residual radial strains f . . . . . . . . . . 146

B.4 Comparison of outer sheet residual tangential strains . . . . . . . . . . 147

B.5 Comparison of inner sheet residual tangential strains . . . . . . . . . 147

B.6 Comparison of outer sheet residual clamping strains $\ldots \ldots \ldots \ldots$

B.7 Comparison of inner sheet residual clamping strains . . . . . . . . . . 148

C.1 Schematic of rivet installation finite element model . . . . . . . . . . . 149

C.2 2024-T3 residual stress results for $F_{S q}=1000 \mathrm{lbf} \ldots \ldots \ldots \ldots$

C.3 2024-T3 residual stress results for $F_{S q}=1500 \mathrm{lbf} \ldots \ldots \ldots \ldots$

C.4 2024-T3 residual stress results for $F_{S q}=2000 \mathrm{lbf} \ldots \ldots \ldots \ldots \ldots$

C.5 2024-T3 residual stress results for $F_{S q}=2500 \mathrm{lbf} \ldots \ldots \ldots \ldots$

C.6 2024-T3 residual stress results for $F_{S q}=3000 \mathrm{lbf} \ldots \ldots \ldots \ldots \ldots$

C.7 GLARE residual stress results for $F_{S q}=1000 \mathrm{lbf} \ldots \ldots \ldots \ldots$

C.8 GLARE residual stress results for $F_{S q}=1500 \mathrm{lbf} \ldots \ldots \ldots \ldots$

C.9 GLARE residual stress results for $F_{S q}=2000 \mathrm{lbf} \ldots \ldots \ldots \ldots$

C.10 GLARE residual stress results for $F_{S q}=2500 \mathrm{lbf} \ldots \ldots \ldots \ldots$

C.11 GLARE residual stress results for $F_{S q}=3000 \mathrm{lbf} \ldots \ldots \ldots \ldots$

D.1 Schematic of splice loading finite element model . . . . . . . . . . . 160

D.2 2024-T3 secondary bending factor results for $F_{S q}=1500 \mathrm{lbf} \ldots \ldots \ldots 16$

D.3 2024-T3 secondary bending factor results for $F_{S q}=2000 \mathrm{lbf} \ldots \ldots \ldots$. . . 162

D.4 2024-T3 secondary bending factor results for $F_{S q}=2500 \mathrm{lbf} \ldots \ldots \ldots$

D.5 2024-T3 secondary bending factor results for $F_{S q}=3000 \mathrm{lbf} \ldots \ldots \ldots \ldots$

D.6 GLARE secondary bending factor results for $F_{S q}=1500 \mathrm{lbf} \ldots \ldots \ldots$

D.7 GLARE secondary bending factor results for $F_{S q}=2000 \mathrm{lbf} \ldots \ldots \ldots \ldots$

D.8 GLARE secondary bending factor results for $F_{S q}=2500 \mathrm{lbf} \ldots \ldots \ldots$

D.9 GLARE secondary bending factor results for $F_{S q}=3000 \mathrm{lbf} \ldots \ldots \ldots$

xvii 
E.1 Mean confidence intervals for outer sheet failure fatigue life . . . . . . 175

F.1 Crack length definition . . . . . . . . . . . . . . . 176

F.2 Location and size of cracks prior to SEM reconstruction . . . . . . . . 177

F.3 Crack front plot for CSK2.0-T1-SEM-01 rivet A2 left side . . . . . . . 178

F.4 Crack front plot for CSK2.0-T1-SEM-01 rivet A2 right side . . . . . . 178

F.5 Crack front plot for CSK2.0-T1-SEM-02 rivet B3 left side . . . . . . . 179

F.6 Crack front plot for CSK2.0-T1-SEM-02 rivet B3 right side . . . . . . . 179

F.7 Crack front plot for CSK2.0-T1-SEM-03 rivet B1 left side . . . . . . . . . 180

F.8 Crack front plot for CSK2.0-T1-SEM-03 rivet B1 right side . . . . . . 180

F.9 Crack front plot for CSK3.0-T1-SEM-01 rivet B3 left side . . . . . . . . 181

F.10 Crack front plot for CSK3.0-T1-SEM-01 rivet B3 right side . . . . . . . 181

F.11 Crack front plot for CSK3.0-T1-SEM-02 rivet B2 left side . . . . . . . . 182

F.12 Crack front plot for CSK3.0-T1-SEM-02 rivet B2 right side . . . . . . . 182

F.13 Crack front plot for CSK3.0-T1-SEM-03 rivet A1 left side . . . . . . . 183

F.14 Crack front plot for CSK3.0-T1-SEM-03 rivet A1 right side . . . . . . . . 183

xviii 


\title{
Nomenclature
}

\author{
Abbreviations
}

ANOVA ANalysis Of VAriance

FE Finite element

FML Fibre Metal Laminate

FTI Fatigue Technology Inc.

GLARE GLAss REinforced aluminum laminate

NACA National Advisory Committee for Aeronautics

prepreg Epoxy pre-impregnated fibre composite

\section{Greek Symbols}

$\begin{array}{ll}\epsilon_{\theta} & \text { Tangential strain } \\ \epsilon_{r} & \text { Radial strain } \\ \epsilon_{\text {true }} & \text { True strain } \\ \theta & \text { Sheet orientation angle with respect to sheet rolling direction } \\ \sigma_{\theta} & \text { Tangential stress } \\ \sigma_{\text {applied }} & \text { Applied stress } \\ \sigma_{\text {bending }} & \text { Secondary bending stress } \\ \sigma_{\text {fay }} & \text { Faying joint surface stress } \\ \sigma_{\text {free }} & \text { Free joint surface stress } \\ \sigma_{r} & \text { Radial stress }\end{array}$

xix 


$\begin{array}{ll}\sigma_{y} & \text { Yield stress } \\ \nu & \text { Global Poisson's ratio } \\ \nu_{21} & \text { Local } 21 \text { Poisson's ratio } \\ \nu_{32} & \text { Local } 32 \text { Poisson's ratio } \\ \nu_{13} & \text { Local 13 Poisson's ratio }\end{array}$

\section{Latin Symbols}

\begin{tabular}{ll}
$A l 1, A l 2$ & $1^{\text {st }}$ and $2^{\text {nd }}$ aluminum layers with a GLARE laminate \\
$D$ & Driven rivet head diameter \\
$D_{o}$ & Uninstalled rivet shank diameter \\
$E$ & Global elastic modulus \\
$E_{11}$ & Local 11 elastic modulus \\
$E_{22}$ & Local 22 elastic modulus \\
$E_{33}$ & Local 33 elastic modulus \\
$f_{\text {rivet }}$ & Rivet flexibility \\
$f_{s h e e t}$ & Sheet flexibility \\
$G_{12}$ & Local 12 shear modulus \\
$G_{23}$ & Local 23 shear modulus \\
$G_{31}$ & Local 31 shear modulus \\
$h$ & Driven rivet head height \\
$h_{o}$ & Length of rivet extended through the rivet grip length \\
$I$ & Second moment of Area \\
$k$ & Strength coefficient for a Power Law plasticity material model \\
$K_{b}$ & Secondary bending factor \\
$M_{x}$ & Secondary bending moment \\
$n$ & Hardening exponent for a Power Law plasticity material model \\
$F_{S q}$ & Rivet squeeze force \\
$p$ & Row pitch \\
\hline &
\end{tabular}

$\mathrm{xx}$ 


$\begin{array}{ll}P & \text { Applied joint running load (load per unit width) } \\ r & \text { Radial position relative to rivet hole centre } \\ r_{y} & \text { Radius of plastic zone around a cold expanded hole } \\ R & \text { Stress ratio } \\ R_{o}, R & \text { Rivet hole radius } \\ S_{\text {max }} & \text { Maximum applied cyclic load } \\ S_{\text {min }} & \text { Minimum applied cyclic load } \\ s & \text { Rivet pitch } \\ t & \text { Sheet thickness } \\ T_{n} & \text { Load transferred by the } \mathrm{n}^{\text {th }} \text { rivet row of a lap joint }\end{array}$

\section{Units}

Metric units where chosen as the primary system of units within this dissertation, in keeping with Canadian and Carleton University standards. The lone exception is the use of the imperial unit of pounds-force, $l b f$, in conjunction with the rivet squeeze force due to its prevalent use in the North American aerospace industry.

xxi 


\section{Chapter 1}

\section{Introduction}

Mechanically fastened joints are an essential part of airframe construction due to limitations in the available sizes of stock material, as well as manufacturing, assembly, and logistical requirements. One fastening method used extensively in aerospace structures is riveting. Riveting utilizes deformable fasteners that are installed by plastically deforming (driving) the fastener shank into the sheets being joined, thereby forming a permanent connection. As a rivet is installed, its shank expands to fill the rivet hole producing an interference which benefits fatigue performance. Other benefits include a low fastener and installation cost.

Despite the benefits associated with rivets and riveting, the use of dated rivet installation guidelines and techniques is limiting the true potential of riveting with respect to fatigue performance. The quality of installed rivets is typically assessed by the size and shape of the driven rivet head. Large bounds on acceptable driven rivet head geometries have been established primarily from experience and the limitations of early hand riveting techniques, leading to a large variability in rivet installation and the resulting interference. Advances in riveting technology have resulted in the development of pneumatic rivet squeezers and fully automated force-controlled rivet squeezers which offer greater control over the rivet installation process. However, the use of old 'rules of thumb' regarding acceptable driven rivet head geometries continues.

Taking advantage of the greater control offered by modern force-controlled riveting tools provides the opportunity to re-examine rivet installation and exploit its effects. Some of 
these effects have already been proven. Müller [1] demonstrated a link between the rivet installation force, or squeeze force, and the overall fatigue life of a riveted joint. The squeeze force, he argued, is more directly linked to the resulting rivet interference than the driven rivet head geometry, making it a superior measure of installation quality. However, practical limits on rivet head geometry must remain.

Rivet interference, however, is not the only consequence of rivet installation that affects fatigue. Decades of experience with the fatigue performance of riveted joints within the design and research communities have resulted in many experimental observations. Variations in crack nucleation times and locations, fatigue crack growth rates and paths, and overall fatigue lives have all been observed in seemingly identical specimens. These variations are typically attributed to fatigue scatter, however, some of the observations, such as the location of crack nucleation, have been loosely associated with observed differences in driven rivet head diameters and thus rivet installation. Indeed, it is clear that the performance of a fatigue sensitive detail such as a fastener location should be dependent on the state of that fastener. If this sensitivity is well understood, it is possible that it could be exploited.

Recent advances in airframe materials provide additional motivation for re-examining the rivet installation process. New hybrid laminates known as Fibre Metal Laminates (FMLs) are gaining acceptance in the aerospace industry and will achieve their first commercial primary structural application in the upper fuselage of the Airbus A380. It is plausible that differences between such new materials and monolithic aluminum alloys may result in different optimum rivet installations, and that riveting guidelines and parameters based on material behaviour could be developed.

\section{$1.1 \quad$ Research Objective}

Based on the above motivation, a research project aimed at investigating the role of rivet installation on the fatigue performance of riveted lap joints was initiated. To understand the fatigue process within riveted lap joints, detailed knowledge of the stress state is required. Factors contributing to this stress field include: 
- Bulk tensile stress exacerbated by stress concentrations at the rivet hole

- Bearing stresses due to load transfer through the rivet

- Frictional forces and fretting damage

- Secondary bending stresses resulting from joint eccentricities

- Residual stresses resulting from rivet interference

Examining each of these factors in detail was considered too broad a scope for the current investigation. Based on the work conducted by Müller, rivet interference and the formation of residual stresses were defined as a key focus for the current research. Due to the link between secondary bending and crack nucleation, the role of rivet installation on the secondary bending stress field was also defined as a key focus. To this end, the following research objective was formulated:

\section{To develop a better understanding of the role of rivet installation on the fatigue performance of riveted lap joints by studying, through numerical and experimental means, the effects of process variations on the formation of residual stresses, distribution of secondary bending stresses, and on crack growth.}

In addition to examining the role of rivet installation on fatigue performance, the role of sheet material is also examined. The potential for applications of fibre metal laminates in future airframe structures increases the likelihood for FML specific lap joint designs. One variant of FML, known as GLARE (GLAss REinforced aluminum), is currently gaining acceptance as a replacement for monolithic aluminum in airframe structures. For this reason, both monolithic 2024-T3 aluminum, the current standard for fatigue critical airframe structures, and GLARE, a potential future standard, are examined in this research. 


\subsection{Dissertation Overview}

This dissertation presents the results of a numerical and experimental investigation into the role of rivet installation on the fatigue performance of riveted lap joints.

Chapter 2 provides a brief overview of the current state-of-the-art in riveting practice and lap joint design. This chapter serves to provide additional motivation for the research carried out and the necessary background information to interpret and apply the results.

In Chapters 3 and 4, finite element studies into the formation of residual stresses are presented. First, the cold expansion process commonly used to introduce residual stresses in mechanically fastened joints is studied. This process is simpler and better understood in terms of residual stress than riveting, and provides the opportunity to study residual stress formation on a more fundamental level. Subsequently, the formation of residual stress due to rivet installation is studied and compared to the cold expansion process.

Chapter 5 switches focus from residual stress to secondary bending. Using finite element methods, the influence of rivet type and installation on the local secondary bending stress field are studied. Results are compared to existing analytical models currently used for secondary bending predictions.

In Chapter 6, experimental methods are employed to study the effects of the residual and secondary bending stress fields observed in the previous chapters on crack nucleation, growth, and the overall fatigue life of riveted lap joints. A novel fatigue spectrum is used to create markers on the fracture surface of lap joint specimens, permitting fractographic reconstructions of the fatigue life using a scanning electron microscope (SEM).

Finally, an overall discussion of the results and set of conclusions are provided in Chapters 7 and 8. 


\section{Chapter 2}

\section{Background}

\subsection{Introduction}

The riveted single shear lap joint, illustrated in Figure 2.1, is one of the most common joint configurations used in metallic aircraft construction due to its relative simplicity and low-weight. These positive attributes come at the cost of a reduced fatigue performance compared to single- and double-shear butt joint configurations; however, it is commonly accepted that through proper design, a lap joint can offer sufficient fatigue life for most applications. Efficient design and optimization of riveted lap joints is not a trivial task. A combination of complex load transfer mechanisms, bending due to joint eccentricities (secondary bending), and residual stresses resulting from rivet installation complicate the design process which still relies heavily on the experience of the designer and empirical testing.

The remainder of this chapter will review and summarize the design considerations related to the fatigue performance of riveted lap joints for airframe applications. As mentioned in Chapter 1, two material types are considered in the present investigation; aluminum 2024-T3 Clad and GLARE. To facilitate the comparison of these two material types, a review of GLARE as a member of the fibre metal laminate family of materials will also be presented. 


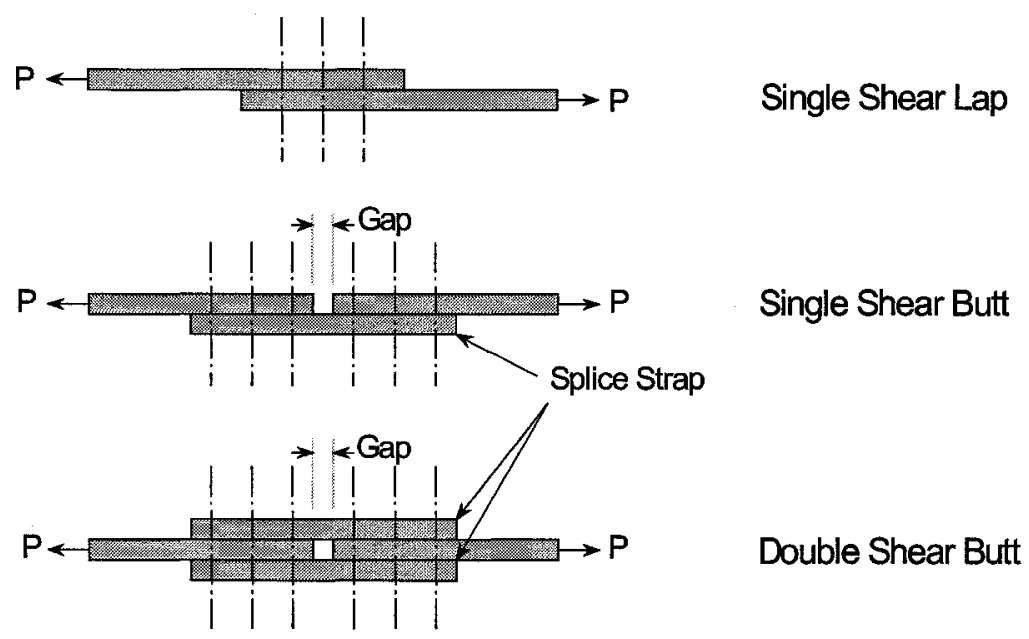

Figure 2.1: Types of tension loaded riveted joints

\subsection{Solid Rivets}

Rivets are a class of permanent mechanical fasteners that are installed by plastically deforming one or both ends of the fastener. Blind rivets are a sub-class of rivets that can be installed with access to only one side of the work piece and typically consist of some form of tubular fastener through which an oversized mandrel is pulled (Figure 2.2). Solid rivets, conversely, are another sub-class of rivets requiring access to both sides of the work piece during installation and consist of a solid fastener that is deformed under a compressive forming load. Due to their low cost and relatively good fatigue performance, solid rivets are extensively used in the aerospace industry. Only solid rivets and the fatigue performance of joints incorporating solid rivets will be considered here. Further use of the term rivet will refer to solid rivets unless otherwise stated.

\subsubsection{Rivet Installation}

Rivet installation consists of a forging process whereby the rivet shank is deformed under compressive load; thus constraining the fastened parts between the manufactured and driven rivet heads. Application of the compressive load can be accomplished through a hammering or squeezing process. Hammering is typical of hand riveting practices where pneumatic 


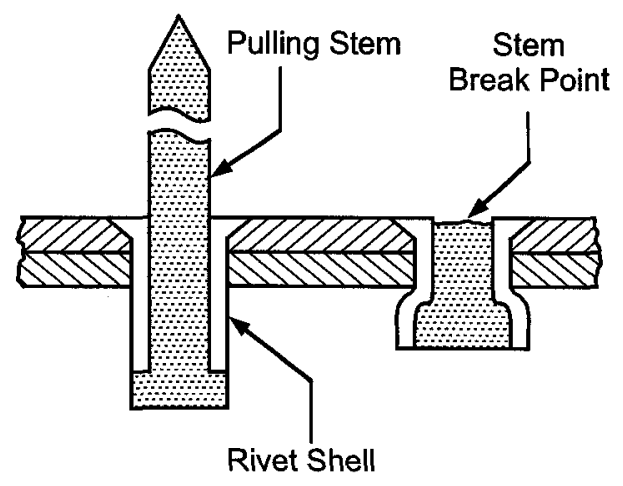

Blind Rivet

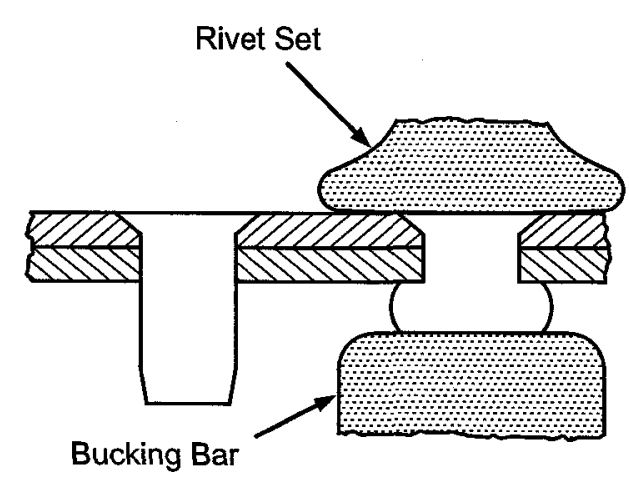

Solid Rivet

Figure 2.2: Blind and solid rivets

riveting guns are used to progressively deform the rivet shank to the desired dimensions. Many of the guidelines and general practices surrounding riveting evolved through the experiences with and limitations of hand riveting. As technology advanced, large automated riveting gantries which utilize a squeezing action were developed. Whether displacement controlled or force controlled, rivet squeezing provides a greater degree of control than hand riveting practices, resulting in a more consistent rivet installation. An installed rivet and nomenclature associated with rivet installation are illustrated in Figure 2.3.

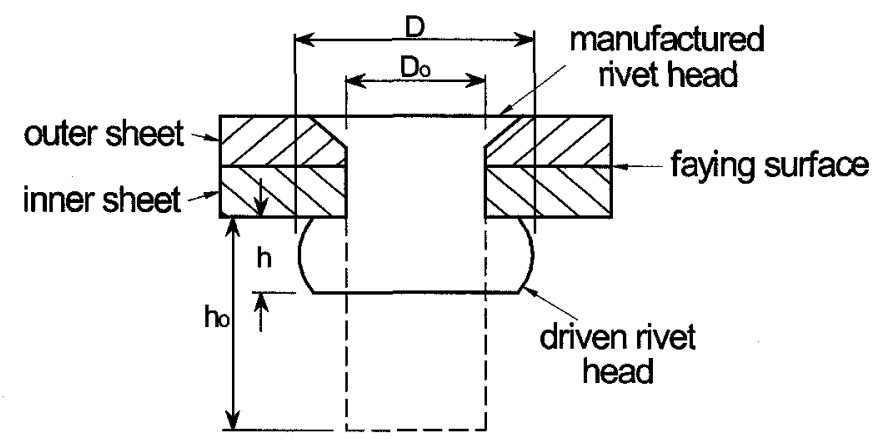

Figure 2.3: Rivet installation nomenclature

Rivet installation can be divided into four distinct stages (Figure 2.4):

1. Hole preparation and rivet insertion. A hole with a diameter approximately $0.1 \mathrm{~mm}$ larger than the rivet diameter is drilled in the components being joined. Since the 

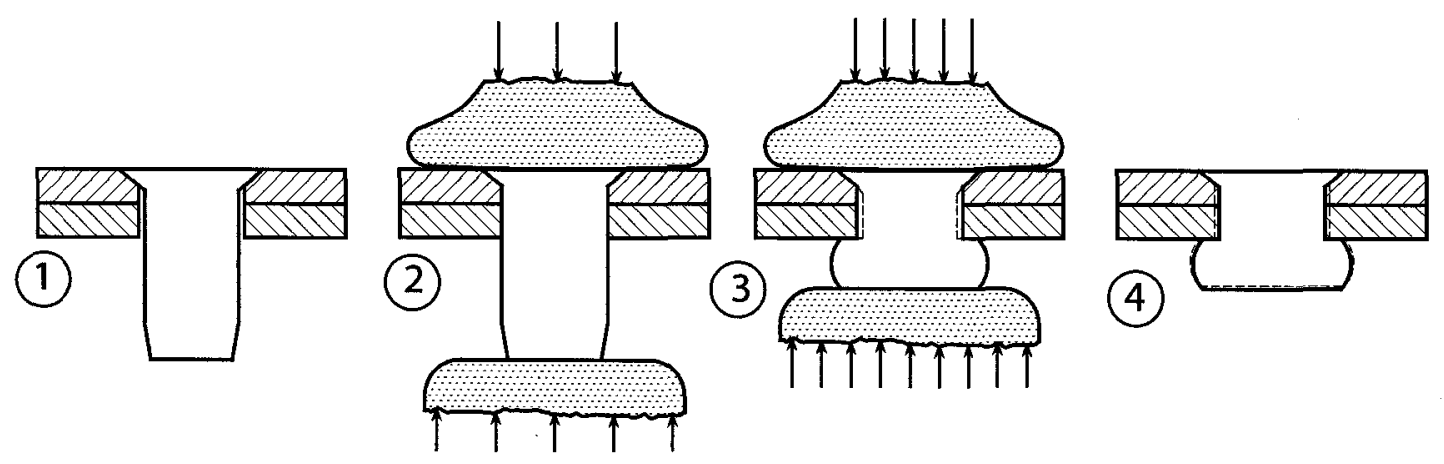

Figure 2.4: Stages of rivet squeezing [1]

rivet expands during installation to fill the rivet hole, this oversized hole diameter allows for lower tolerances on the hole dimensions, keeping installation costs down. The hole is then deburred and countersunk (if required), then the rivet is inserted for installation.

2. Rivet expansion. As the rivet is compressed, the rivet shank expands to fill the rivet hole and begins to exert a radial pressure on the sheet material. At this stage, the rivet material is already plastically deformed while deformation of the sheet material is elastic. Due to the resistance provided by the sheet material along the shank of the rivet, the free end of the rivet begins to expand at a greater rate forming the driven rivet head.

3. Sheet expansion. Further expansion of the rivet shank results in plastic deformation of the sheet material adjacent to the rivet hole. As the driven rivet head continues to form it comes into contact with the sheet material. As a result, a portion of the squeeze force begins to transfer directly through the mating sheets and contributes to further plastic deformation of the sheet material. This process proceeds until the desired squeeze force is reached.

4. Springback. The final stage consists of removal of the squeeze force. During this stage, elastic springback of the rivet and surrounding sheets occurs. The rivet relaxes reducing its diameter, increasing its length, and increasing the height of the driven 
rivet head. The sheets relax in a similar manner. Subsequent to springback, a residual stress state is left within the rivet-sheet system. The residual stress state plays a key role in the fatigue performance of riveted joints and will be further discussed in Section 2.4 .

Traditionally, rivet installation has been assessed by the ratio of the driven rivet head diameter to the original rivet shank diameter $\left(D / D_{o}\right)$. In addition, qualitative inspections of the driven rivet head shape are performed to identify improper alignment of riveting tools. Standard practices call for a nominal $D / D_{o}$ ratio of 1.5 with an acceptable tolerance range of 1.3-1.8. The ratio of the driven rivet height to rivet shank diameter $\left(h / D_{o}\right)$ is used as a check on proper rivet length selection, with a nominal value of 0.5 [1]. These constraints were developed based on limitations in repeatability of hand riveting practices; however, they have also carried over to rivet squeezing practices.

An alternative measure possible for rivet squeezing practices is the squeeze force $\left(F_{S q}\right)$. Müller [1] studied the effects of rivet installation on the fatigue performance of riveted lap joints and found that variations in $D / D_{o}$ (and consequently $F_{S q}$ ) had a significant effect on overall fatigue life. Müller attributed this effect to variations in residual stress fields formed around the rivet hole during installation and argued that the squeeze force was a superior measure of installation quality as it could be more directly linked to the residual stress field than the driven rivet geometry. This view is adopted in this study, meaning force controlled riveting practices will be used and the rivet squeeze force will be the primary measure of installation quality. Driven rivet geometry and the $D / D_{o}$ ratio will be used as a secondary quality measure.

\subsubsection{Rivet Type and Geometry}

Within the category of solid rivets, several types and styles of rivets exist (Figure 2.5). In applications where aerodynamic smoothness is not a concern, protruding head rivets are commonly used. Although several head styles exist for protruding rivets, the so-called universal head style is most prevalent. In applications where aerodynamic smoothness is a 


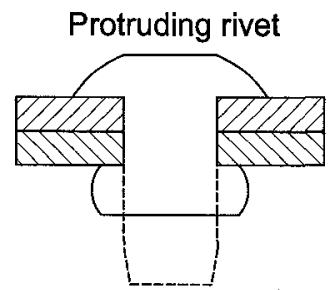

NACA rivet

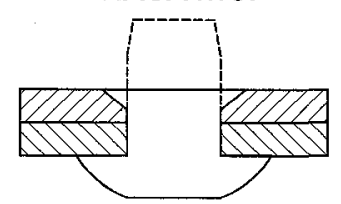

Countersunk rivet (machine countersunk)

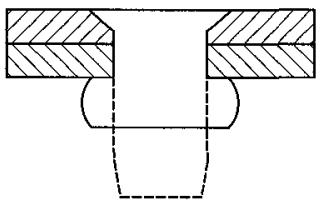

Briles rivet

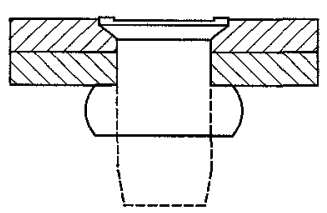

Countersunk rivet

(dimple countersunk)

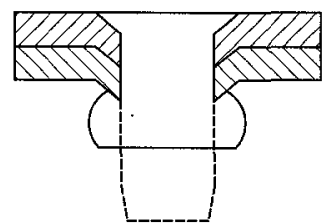

Slug rivet

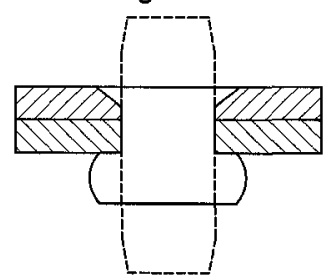

Figure 2.5: Types of solid rivets [1]

concern, countersunk rivets are used. These rivets require the added step of countersinking which can be accomplished in one of two ways. For sheets with a thickness less than $1 \mathrm{~mm}$, the sheets can be plastically deformed to form the countersink in a process known as dimpling. For sheets with a thickness greater than or equal to $1 \mathrm{~mm}$, countersinks can be formed by machining away excess material. The basic aerospace countersunk rivet has a $100^{\circ}$ conical head, regardless of the countersinking method, although various countersink depths are available.

Several specialized rivet types also exist. The NACA rivet is installed with the driven rivet head in the countersunk sheet. This type of installation insures that the countersunk rivet head properly fills the countersunk sheet, minimizing rivet tilting during loading and providing an increased interference in the fatigue critical countersunk sheet. Installation of NACA rivets does not ensure a sufficiently flush surface, making an additional milling operation on the driven head necessary (rivet shaving). The Briles rivet contains a recessed countersunk head which is expanded during installation using a specialized rivet set. This added expansion of the countersunk head provides the same benefits as the NACA rivet. Finally, the slug rivet consists of a cylindrical slug of material that is deformed at both ends during installation. This type of rivet is an attempt at reducing the cost of riveting 
by allowing the use of rivet wire instead of individual rivets. As with NACA rivets, rivet shaving may be required with slug rivets to achieve the desired aerodynamic smoothness.

Rivets are identified by their head style, alloy, diameter, and length using an identification code system. The code breakdown is as follows:

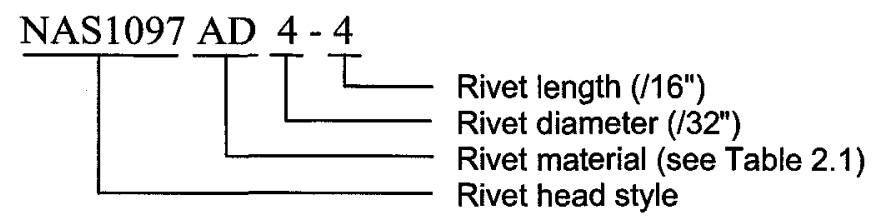

Various rivet alloys are available as shown in Table 2.1.

Selection of rivet length and diameter is determined by several rules of thumb $[1,2]$. The rivet length should nominally equal the combined thickness of the joined sheets plus $1.5 D_{o}$. In cases where the combined sheet thickness is greater than $7.0 \mathrm{~mm}$, the rivet length should be increased by an extra $1.0 \mathrm{~mm}$. Rivet diameter should also be selected such that $D_{o}$ is greater than three to four times the combined sheet thickness.

Table 2.1: Rivet aluminum alloy designations [1]

\begin{tabular}{cc}
\hline Material Designation & Rivet alloy \\
\hline A & 1100 \\
B & $5056-\mathrm{H} 32$ \\
AD & $2117-\mathrm{T} 4$ \\
D & $2017-\mathrm{T} 4$ \\
DD & $2024-\mathrm{T} 4$ \\
E & $7050-\mathrm{T} 73$ \\
\hline
\end{tabular}

\subsection{Lap Joint Nomenclature}

The basic riveted lap joint configuration consists of two sheets joined by one or more rows of rivets as shown in Figure 2.6. The sheets are differentiated by their location and accessibility for inspection along the joint overlap; in an aircraft fuselage application, the outer sheet would be accessible from the outside of the fuselage while the inner sheet would be accessible 
from inside the fuselage. Spacing of the rivets is defined by the rivet pitch, $s$, within each rivet row, and the row pitch, $p$, between each rivet row for multi-row joints. Additional variables and nomenclature are defined in Figure 2.6. The fuselage curvature is omitted for clarity.
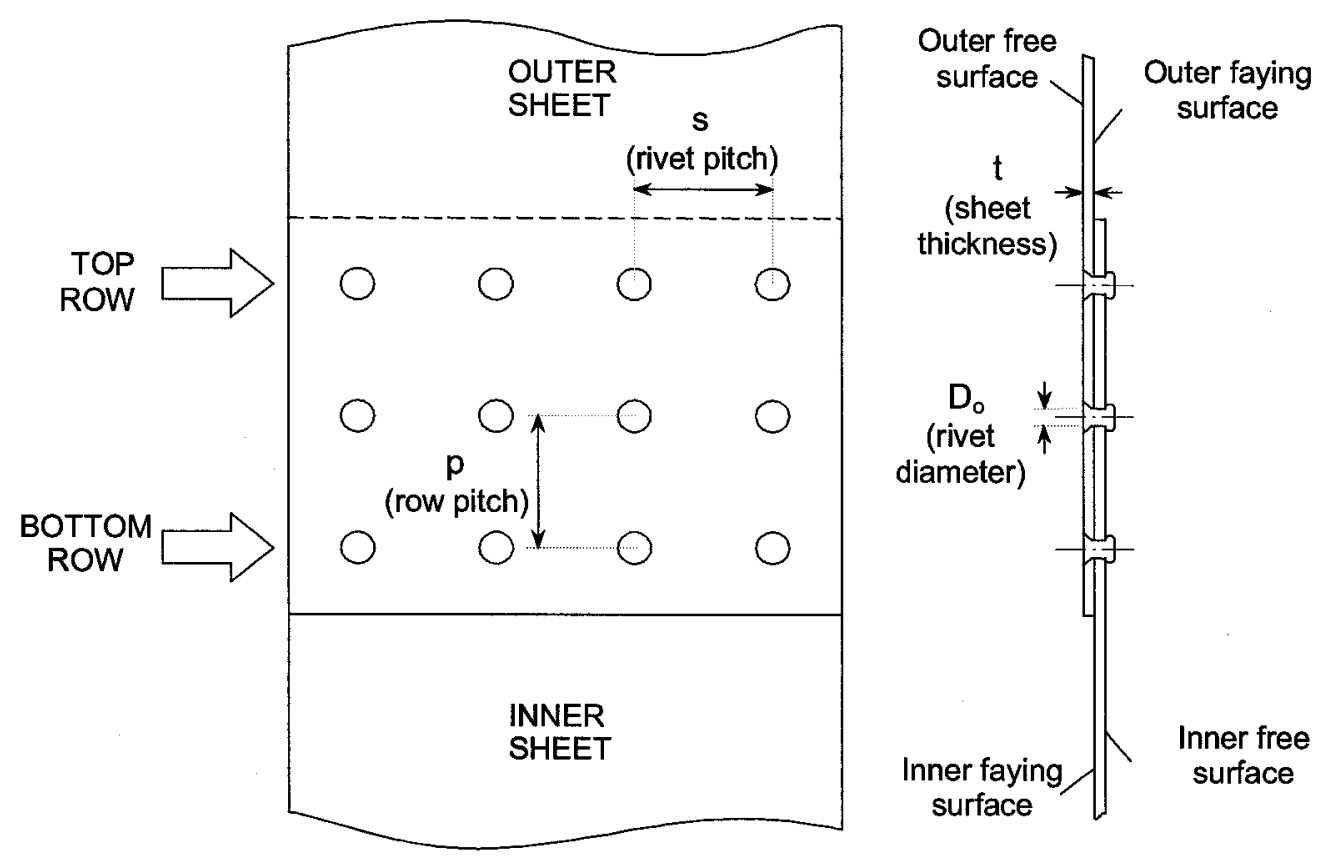

Figure 2.6: 3-row riveted lap joint nomenclature

\subsection{Local Stress Field in a Loaded Lap Splice}

Accurate prediction of fatigue life and performance of riveted lap joints requires an understanding of the local stress field surrounding each rivet. This stress field can be broken down into components related to load transfer (bearing, bypass, and frictional loads) and secondary loads (secondary bending and rivet interference) as illustrated in Figure 2.7. A brief review of each of these components is necessary in order to study the influence of force-controlled rivet installation on the fatigue performance of riveted lap joints. 


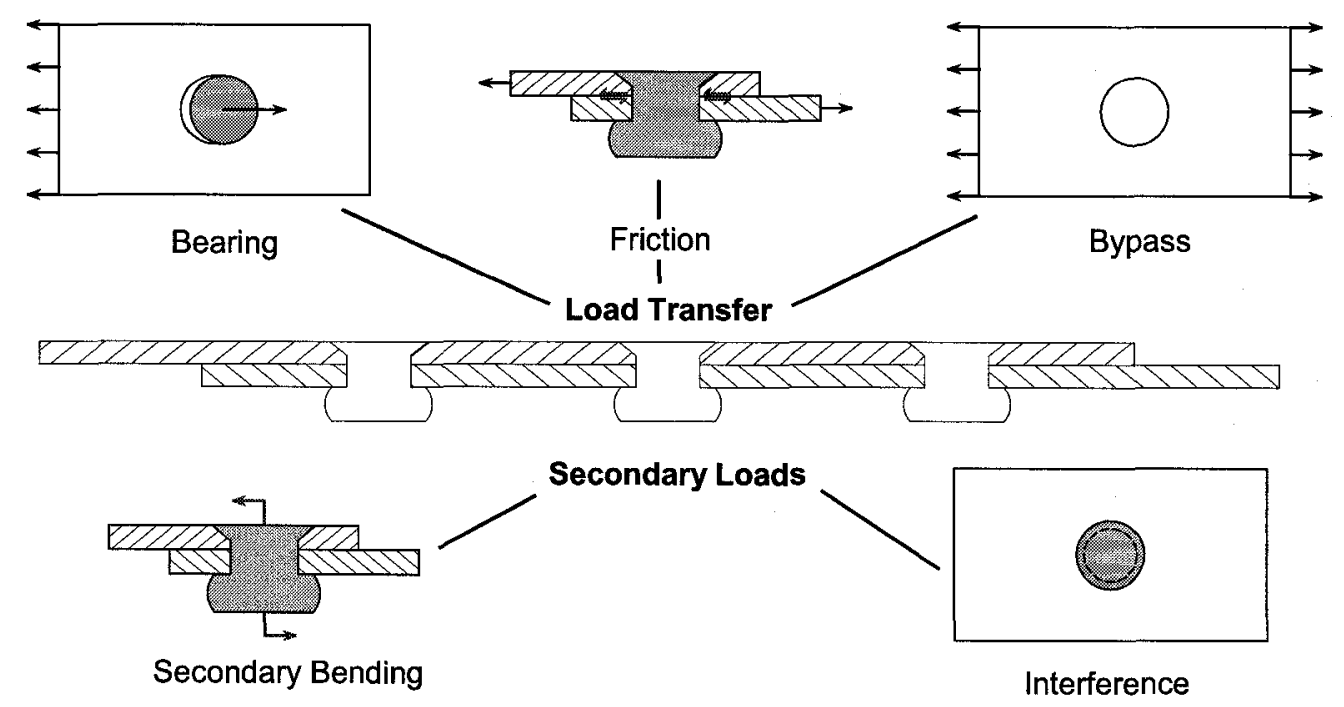

Figure 2.7: Illustration of local stress field components in a riveted lap joint

\subsubsection{Load Transfer}

Load transfer in riveted lap joints occurs by two mechanisms. The majority of load is transferred by means of bearing between the rivets and inner and outer sheets while the remainder is transferred through friction between the faying joint surfaces. Friction comprises a small portion of the overall load transfer and is often neglected; however, fretting damage resulting from this frictional load transfer can be a contributing factor to fatigue crack nucleation. In multiple-row lap joints, each rivet row transfers a portion of the applied load from the outer to inner sheet while the remainder (referred to as the bypass load) remains in the outer sheet. The amount of load transferred by each rivet row is related to the relative flexibilities of the rivets and sheets being joined. Using displacement compatibility and neglecting frictional load transfer, the relative load transferred by each rivet row in a 3-row lap joint can be determined [1]:

$$
\begin{gathered}
T_{1}+T_{2}+T_{3}=P \\
T_{1}=T_{3}
\end{gathered}
$$




$$
\frac{T_{2}}{T_{1}}=\frac{f_{\text {rivet }}}{f_{\text {rivet }}+f_{\text {sheet }}}
$$

where:

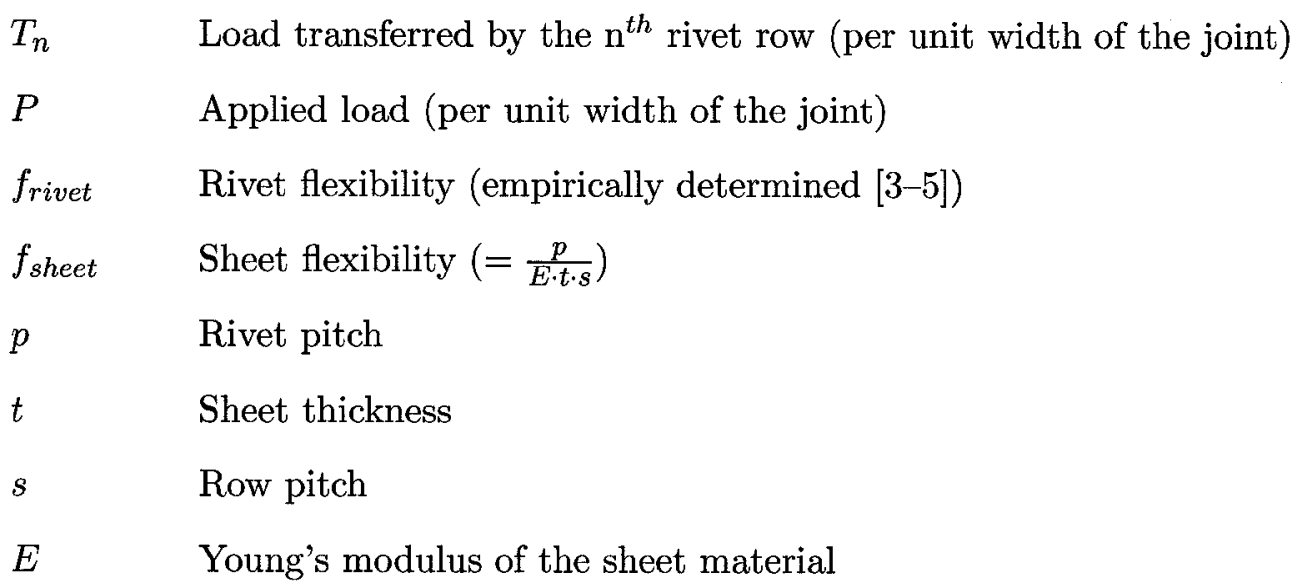

As negative flexibilities are not possible, equation 2.3 shows that the top and bottom rivet rows will always transfer (through bearing) a larger percentage of the load than the middle rivet rows. The combination of large bearing stresses and high bypass stresses along these rivet rows results in two fatigue critical locations: in the outer sheet along the top rivet row and in the inner sheet along the bottom rivet row (Figure 2.8). In practice,

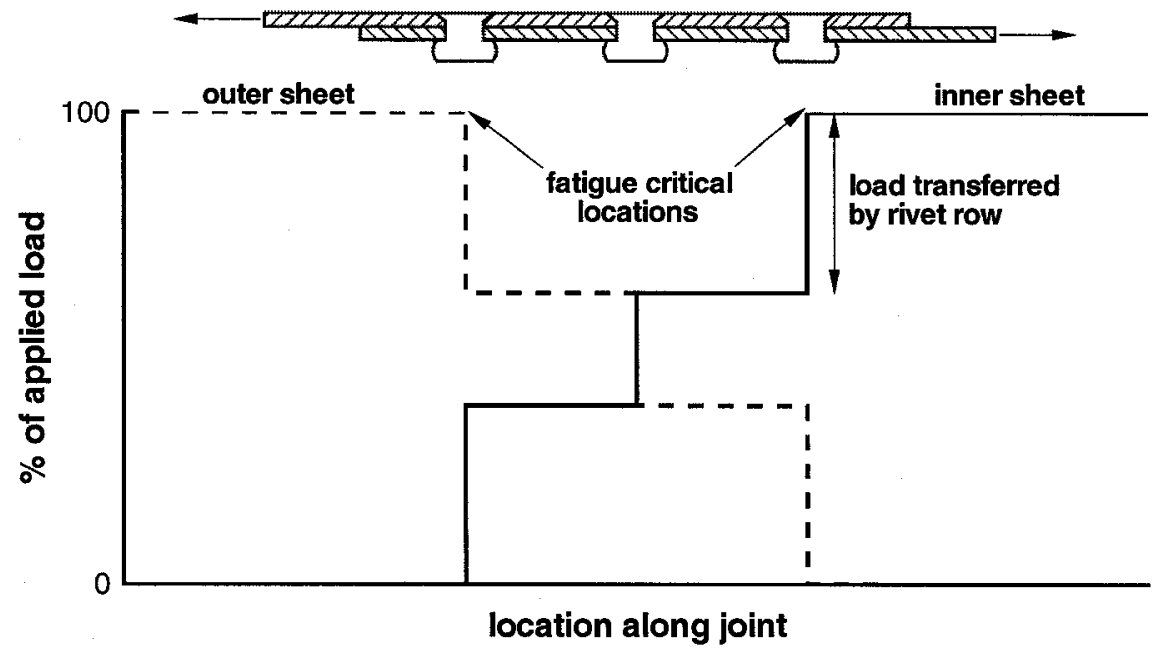

Figure 2.8: Illustration of load carried by inner and outer sheets in a 3-row lap joint 
the inner sheet location is typically less critical due to improved hole filling within this sheet during riveting and due to the added stress concentration in the outer sheet due to countersinking in the case of a countersunk joint. Although adding more rows of rivets beyond three can reduce the amount of load transferred through the top and bottom rivet rows, this reduction drops off rapidly with the number of rivet rows and is typically viewed as a weight and manufacturing penalty.

Studies conducted by Müller [1] demonstrated that the level of interference in the inner and outer joint sheets as a result of rivet installation significantly affects rivet flexibility. For rivets installed with a low squeeze force, a loss in rivet-sheet interference at low applied loads could double the rivet flexibility and alter load distribution. Available empirical formulas for rivet flexibility do not account for this effect. Rather, it is assumed that the riveting procedure used for the experimental coupons is representative of production procedures.

Rivet installation also influences frictional load transfer and fretting fatigue. It is generally agreed that installing rivets with higher squeeze forces results in higher rivet clamping force; however, there are conflicting studies on the relative magnitudes and significance of the rivet clamping force $[1,6,7]$. Additional clamping resistance is provided by the driven rivet head as load is applied to the joint and out-of-plane deformation occurs around the rivet due to load-path eccentricities. Larger driven rivet heads provide increased resistance to this deformation resulting in larger clamping forces, increased frictional load transfer, and a higher incidence of fretting fatigue.

\subsubsection{Secondary Bending}

Due to the presence of load path eccentricities, out-of-plane displacements and additional bending stresses are present in a loaded lap joint. Known as secondary bending, this mechanism increases the peak tensile stresses encountered along the faying joint surfaces near rivet locations and is therefore important in terms of fatigue performance. Prediction of secondary bending moments and stresses can be accomplished by modelling the joint by its neutral axis (Figure 2.9) $[1,2,8]$, giving: 


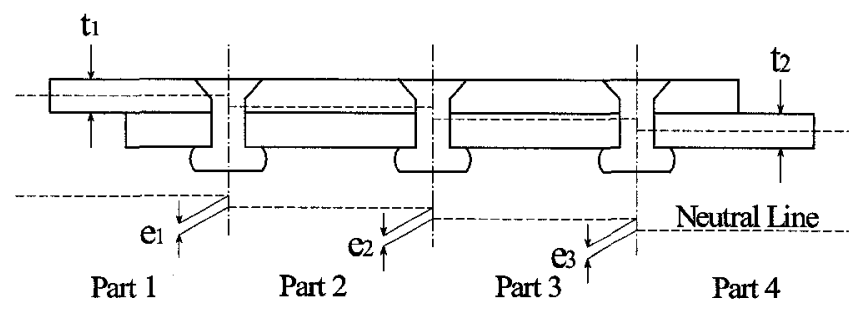

Figure 2.9: Neutral line representation of a three-row riveted lap joint [8]

$$
M_{x}=P \cdot w=E_{i} I_{i} \cdot \frac{d^{2} w}{d x^{2}} \Rightarrow \frac{d^{2} w}{d x^{2}}-\alpha^{2} \cdot w=0 ; \quad \alpha^{2}=\frac{P}{E_{i} I_{i}}
$$

which has the following solution:

$$
w_{i}=A_{i} \cdot \sinh \left(\alpha_{i} \cdot x\right)+B_{i} \cdot \cosh \left(\alpha_{i} \cdot x\right)
$$

where: $M_{x} \quad$ Secondary bending moment

$P \quad$ Applied load (per unit width of the joint)

$w \quad$ Out-of-plane displacement of sheet

$E_{i} I_{i} \quad$ Flexural rigidity of part i (Figure 2.9)

$A_{i}, B_{i} \quad$ Constants related to joint boundary conditions

Assuming a pinned boundary condition at the loaded ends of the joints, constants $A$ and $B$ can be determined, allowing secondary bending stresses to be determined by:

$$
\sigma_{\text {bending }}=\frac{M_{x} \cdot z}{I}=\frac{P \cdot w(x) \cdot z}{I}=w(x)^{\prime \prime} \cdot E \cdot z
$$

where $\mathrm{z}$ is the distance from the neutral axis.

The severity of the secondary bending is typically quantified by the secondary bending factor, $K_{b}$. This bending factor is defined as the ratio of the bending stress and the remotely applied stress:

$$
K_{b}=\frac{\sigma_{\text {bending }}}{\sigma_{\text {applied }}}
$$


The primary limitation of the neutral line model is its inability to account for the variation in secondary bending stress distribution around the installed rivet. The neutral line model predicts that the maximum secondary bending stresses occur along the net section of the rivet row, whereas in reality, they flow around the driven and manufactured rivet heads (Figure 2.10). Experimental observations have shown that this effect is dependant on the size of the driven rivet head (and thus rivet squeeze force) and can shift the critical crack initiation location from the net section of the rivet row to the perimeter around the driven rivet head [1].

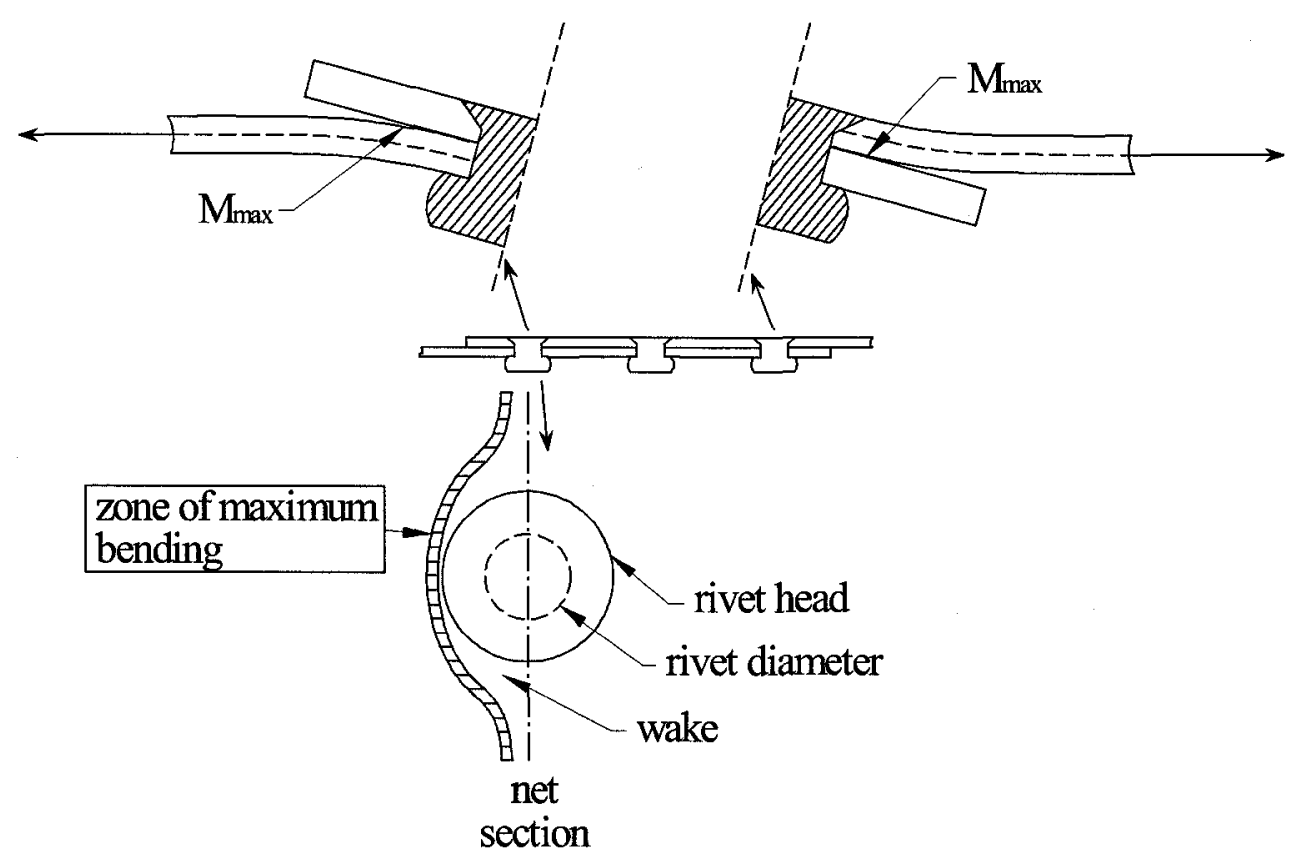

Figure 2.10: Influence of rivet geometry on location of peak secondary bending stress [1]

\subsubsection{Rivet Interference}

Expansion of the rivet shank during installation produces an interference that results in a beneficial residual stress field within the sheet material surrounding the rivet hole. This process is analogous to cold expansion processes where fastener holes are expanded to a desired level before inserting a mechanical fastener; however, in the case of rivet installation, a portion of the springback is prevented by the presence of the rivet. Considering a uniform 
expansion process in an infinite elastic-perfectly-plastic plate containing a hole with radius $R_{o}$, an expression for the residual stress distribution can easily be derived. Under an applied pressure, $p$, the material deforms elastically such that the radial $\left(\sigma_{r}\right)$ and tangential $\left(\sigma_{\theta}\right)$ stresses are given in the radial direction, $r$, by:

$$
\sigma_{r}=-p\left(\frac{R_{o}}{r}\right)^{2}, \quad \sigma_{\theta}=p\left(\frac{R_{o}}{r}\right)^{2}
$$

As the pressure, $p$, is increased, yielding will occur in a circular region bound by $R_{o} \leq$ $r \leq r_{y}$. Using the Tresca yield criterion, Park and Atluri [9] derived expressions for the stress distribution with this plastic deformation:

$$
\begin{gathered}
\sigma_{r}=-p+\sigma_{y} \ln \left(\frac{r}{R_{o}}\right), \quad R_{o} \leq r \leq r_{y} ; \quad \sigma_{r}=-\frac{\sigma_{y}}{2} \cdot\left(\frac{r_{y}}{r}\right)^{2}, \quad r>r_{y} \\
\sigma_{\theta}=\left(\sigma_{y}-p\right)+\sigma_{y} \ln \left(\frac{r}{R_{o}}\right), \quad R_{o} \leq r \leq r_{y} ; \quad \sigma_{\theta}=\frac{\sigma_{y}}{2} \cdot\left(\frac{r_{y}}{r}\right)^{2}, \quad r>r_{y}
\end{gathered}
$$

where $\sigma_{y}$ is the yield stress of the plate material. The radius of the plastic region, $r_{y}$, is related to the radial pressure, $p$, by:

$$
p=\sigma_{y} \cdot\left[\frac{1}{2} \cdot \ln \left(\frac{r_{y}}{R_{o}}\right)\right]
$$

The residual stress distribution after removal of pressure, $p$, can be obtained by subtracting the elastic stress distributions of Eq. 2.8 from the distributions in Eqs. 2.9 and 2.10. A typical set of results is shown in Figure 2.11, which show a region of residual compressive tangential stress near the hole edge.

Additional analytical models for cold expansion processes have been developed [10-12] which account for the effects of various types of strain hardening. All of these models, however, are limited in their applicability to riveting for the following reasons:

- Hole expansion is not uniform through the sheet thickness. 


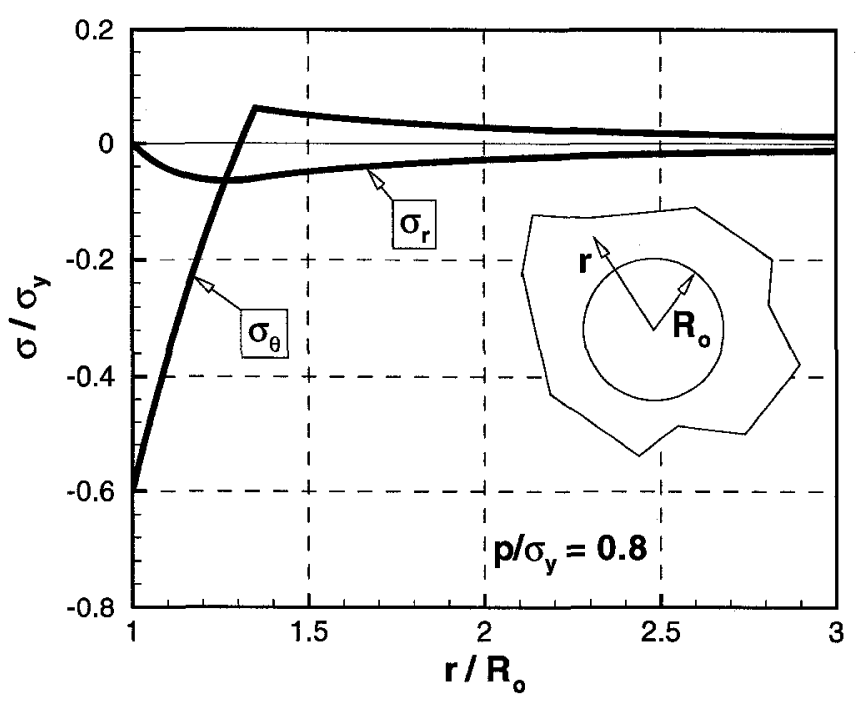

Figure 2.11: Residual stress in an infinite elastic-perfectly-plastic plate expanded by pressure $p$ (calculated using Equations 2.8 through 2.11)

- The manufactured and driven rivet heads exert a pressure through the thickness of the sheet, resulting in a 3 -dimensional stress state.

- The sheet is not free to spring back after expansion. The rivet shank remains in the rivet hole after expansion, resulting in a residual radial pressure at the hole edge.

The residual stresses formed during riveting have a significant bearing on the fatigue performance of riveted joints. Müller [1] demonstrated that simply producing a repeatable residual stress distribution through the use of force-controlled riveting techniques could reduce fatigue life scatter in current lap joint designs. Furthermore, Müller found that the use of larger squeeze forces could increase the fatigue life of 2024-T3 aluminum lap joints 10-fold. These results have sparked interest in exploiting the residual stress state resulting from rivet installation in the design of riveted lap joints, resulting in numerous numerical and experimental studies $[1,6,7,13-18]$. 


\subsection{GLARE Laminates}

Fibre metal laminates (FML's) are a family of hybrid laminates consisting of alternating layers of monolithic metallic sheet and fibre reinforced epoxy pre-impregnated fibre (prepreg) layers (Figure 2.12). Originating from the development of built-up structures pioneered by Fokker Aircraft in the late 1940's, FML's were found to exhibit benefits from both the metallic and fibre reinforced composite constituents. Specifically, FML's possess many of the damage tolerant properties inherent in fibre reinforced composite materials $[1,8,19-21]$ while retaining the fabrication and repair capabilities of sheet metal $[22,23]$. These properties make FML's an attractive option in the aerospace industry, particularly in applications such as airframe construction where increased damage tolerance is desired, but the application of fibre reinforced composites is hindered due to high design, manufacturing, and certification costs. An aluminum-glass fibre class of FML's known as GLARE (GLAss REinforced aluminum) laminates has been gaining acceptance as an alternative to monolithic 2024-T3 sheet in aircraft fuselages, as it is currently used in the Airbus A380.

\subsubsection{GLARE Variants}

GLARE consists of a lay-up of multiple metallic and fibre layers bonded together in an autoclave. Each fibre layer is manufactured using unidirectional prepreg layers consisting of

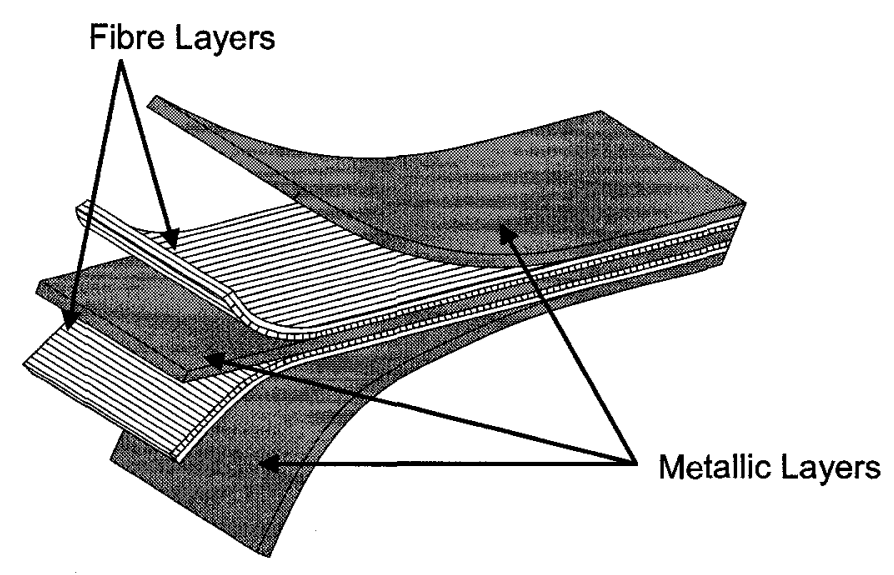

Figure 2.12: Typical fibre metal laminate layup 
S-glass fibres embedded with FM 94 adhesive [24]. By altering the number and orientation of the prepreg layers in each fibre layer of GLARE, a limitless number of GLARE variants is possible. Currently, 6 standard grades are available. These grades are summarized in Table 2.2.

Table 2.2: Standard grades of GLARE [21]

\begin{tabular}{|c|c|c|c|c|}
\hline $\begin{array}{l}\text { GLARE } \\
\text { grade }\end{array}$ & Sub & $\begin{array}{c}\text { Metal sheet } \\
\text { thickness }[\mathrm{mm}] \& \\
\text { alloy }\end{array}$ & $\begin{array}{l}\text { Prepreg } \\
\text { orientation* in } \\
\text { each fibre } \\
\text { layer** }\end{array}$ & $\begin{array}{c}\text { Main beneficial } \\
\text { characteristic }\end{array}$ \\
\hline GLARE 1 & - & $0.3-0.47475-\mathrm{T} 761$ & $0 / 0$ & $\begin{array}{l}\text { fatigue, strength, } \\
\text { yield stress }\end{array}$ \\
\hline \multirow[t]{2}{*}{ GLARE 2} & GLARE 2A & $0.2-0.52024-\mathrm{T} 3$ & $0 / 0$ & fatigue, strength \\
\hline & GLARE 2B & $0.2-0.52024-\mathrm{T} 3$ & $90 / 90$ & fatigue, strength \\
\hline GLARE 3 & - & $0.2-0.52024-\mathrm{T} 3$ & $0 / 90$ & fatigue, strength \\
\hline \multirow[t]{2}{*}{ GLARE 4} & GLARE 4A & $0.2-0.52024-\mathrm{T} 3$ & $0 / 90 / 0$ & $\begin{array}{c}\text { fatigue, strength in } \\
0^{\circ} \text { direction }\end{array}$ \\
\hline & GLARE 4B & $0.2-0.52024-\mathrm{T} 3$ & $90 / 0 / 90$ & $\begin{array}{c}\text { fatigue, strength in } \\
90^{\circ} \text { direction }\end{array}$ \\
\hline GLARE 5 & - & $0.2-0.52024-\mathrm{T} 3$ & $0 / 90 / 90 / 0$ & Impact \\
\hline \multirow[t]{2}{*}{ GLARE 6} & GLARE 6A & $0.2-0.52024-\mathrm{T} 3$ & $+45 /-45$ & $\begin{array}{c}\text { shear, off-axis } \\
\text { properties }\end{array}$ \\
\hline & GLARE 6B & $0.2-0.52024-\mathrm{T} 3$ & $-45 /+45$ & $\begin{array}{c}\text { shear, off-axis } \\
\text { properties }\end{array}$ \\
\hline
\end{tabular}

Along with the 6 standard grades, a standard coding system is used to fully define a GLARE laminate. The code format consists of the grade of GLARE, ratio of metallic-tofibre layers, and metallic layer thickness in mm separated by hyphens. For example:

$$
\text { GLARE3-2/1-0.3 }
$$

would be defined as:

- a GLARE laminate consisting of fibre and aluminum layers conforming to grade GLARE 3 in Table 2.2,

- 2 aluminum layers and 1 fibre layer,

- each aluminum layer is $0.3 \mathrm{~mm}$ thick. 
In some instances, laminates consist of metallic layers with different thicknesses. In such cases, all metallic layer thicknesses are reported in the order of the lay-up. When all metallic layers are the same thickness, the layer thickness is often omitted.

\subsubsection{Fatigue Behaviour}

GLARE laminates exhibit longer fatigue lives than monolithic aluminum sheet due to improved damage tolerance characteristics. The primary feature of GLARE responsible for its improved damage tolerance is the fibre bridging mechanism (Figure 2.13). As cracks form in the aluminum layers, crack opening is resisted by adjacent fibre layers transferring the applied load over the crack. The resulting reduction in crack tip stress intensity factor leads to lower crack growth rates and in some cases impedes further crack growth $[1,20,25-27]$. If fatigue cracks continue to grow, large cyclic shear stresses between the fibre and aluminum layers result in the formation and propagation of a delaminated area. This delamination serves to protect the integrity of the fibre layer by distributing the crack opening strain over a larger area of the fibre layer, further prolonging the life of the material.

The laminated structure of GLARE also provides an improvement to damage tolerance. Delamination of the adhesive between layers prevents the growth of the crack through the thickness of the laminate. As a result, each aluminum layer must undergo crack initiation for a crack to propagate through the entire laminate thickness, improving material damage

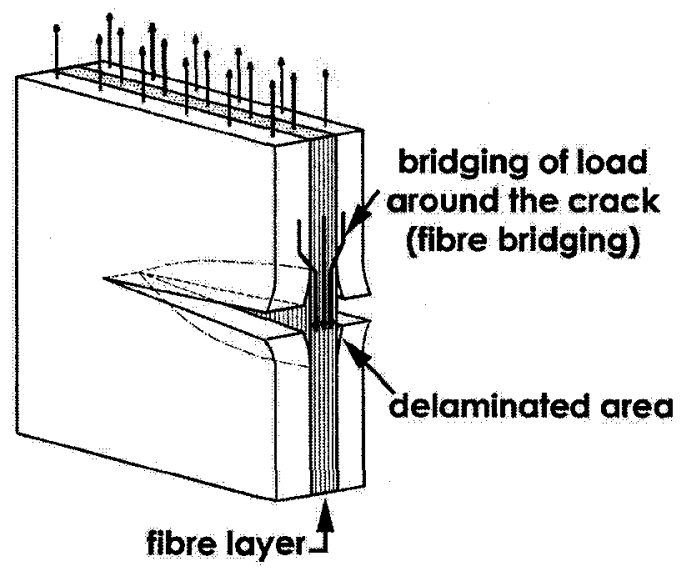

Figure 2.13: Fibre bridging mechanism in GLARE [20] 
tolerance. Furthermore, a plane stress state occurs around the crack tip in laminated structures due to the small thickness of the individual lamina [28].

Despite the improvements in damage tolerance that reduce fatigue crack growth, GLARE laminates exhibit a shorter fatigue crack nucleation period than comparable monolithic aluminum sheet. The fibre bridging mechanism only becomes effective once cracks have grown to sufficient length $(\geq 2 \mathrm{~mm})$ and the fibre layer does not provide any additional mechanism that prevents crack nucleation [25]. Therefore, the fatigue crack nucleation process in GLARE is analogous to that of monolithic aluminum; namely, monolithic aluminum panels and aluminum layers in GLARE with similar stresses will have a similar crack nucleation life. Stress levels in GLARE aluminum layers, however, are higher than in monolithic aluminum sheet of the same thickness for the following reasons:

- The Young's modulus mismatch between the aluminum and fibre layers in GLARE causes stress levels in the stiffer aluminum layers to exceed the gross applied stress.

- The lower bending stiffness of GLARE increases the effects of secondary bending in lap joints.

- Residual tensile stresses in the aluminum layers resulting from thermal expansion during the autoclave curing cycle.

Presently, existing riveted lap joint designs and limits on rivet installation based on experience with monolithic aluminum sheet are applied directly to GLARE. It is the view of the author, however, that as a result of the unique properties of GLARE, optimal joint designs for monolithic aluminum sheet and GLARE laminates may differ. For this reason, the present study considers both monolithic aluminum sheet and GLARE laminates individually.

Within this chapter, a review of additional background information and motivation supporting the need for further investigation into the role of rivet installation on fatigue has been presented. The numerical and experimental study undertaken to meet this need are presented in the following chapters. 


\section{Chapter 3}

\section{Cold Expansion Induced Residual Stresses}

\subsection{Introduction}

Cold expansion processes utilize hardened oversized tooling to expand the diameter of fastener holes, thereby creating radial plastic flow which produces a zone of residual compressive stress around the hole. Although highly simplified compared to the hole expansion that occurs during riveting, the mechanisms involved in the formation of residual stresses in riveting and cold expansion are analogous. These mechanisms have been extensively studied in the context of cold expansion, using experimental and numerical techniques [10-12,29-43], as well as analytically $[10-12,43]$. Given the similarities between riveting and cold expansion, a review of cold expansion processes presents a logical starting point for a study of riveting induced residual stresses.

This chapter provides an overview of various cold expansion techniques and previous investigations into the effects of cold expansion. Results from a finite element study of cold expanded holes in monolithic aluminum and GLARE laminates are also presented. This study provides a means for verifying the performance of finite element techniques to be used in later rivet forming finite element studies against the analytical models which exist for cold expansion processes. Furthermore, the simplicity of the cold expansion process provides an opportunity to investigate potential differences between the formation of residual stresses in monolithic aluminum and GLARE sheets. 


\subsection{Review of Cold Expansion}

Several techniques for achieving cold expansion of a fastener hole exist; however, most are variations of the same basic process. The first step in this basic process is to prepare the starter hole that will be cold expanded. The diameter of this hole will be smaller than that of the nominal fastener hole and will be dependant on the specified level of expansion and the expansion process. The second step is to expand the starter hole by pushing or pulling an oversized hardened component through it. The final step involves further drilling/reaming of the expanded hole to remove any surface artifacts generated on the bearing surface of the hole during cold expansion and to achieve the desired nominal fastener hole size.

Two common cold expansion techniques used in the aerospace industry are the splitmandrel and split-sleeve processes (Figure 3.1).

The split-mandrel process uses a hollow lubricated mandrel that is slotted to allow it to collapse when being inserted into the fastener hole. Once inserted into the hole, the mandrel is expanded and made rigid by inserting a pilot into the hollow portion of the mandrel. The hole is then cold worked by drawing the rigid-expanded mandrel through it.

The split-sleeve process uses a solid mandrel with a major diameter small enough so that it can be passed through the starter hole. A lubricated split-sleeve is placed over the minor diameter of the mandrel and the sleeve/mandrel combination is inserted into the starter

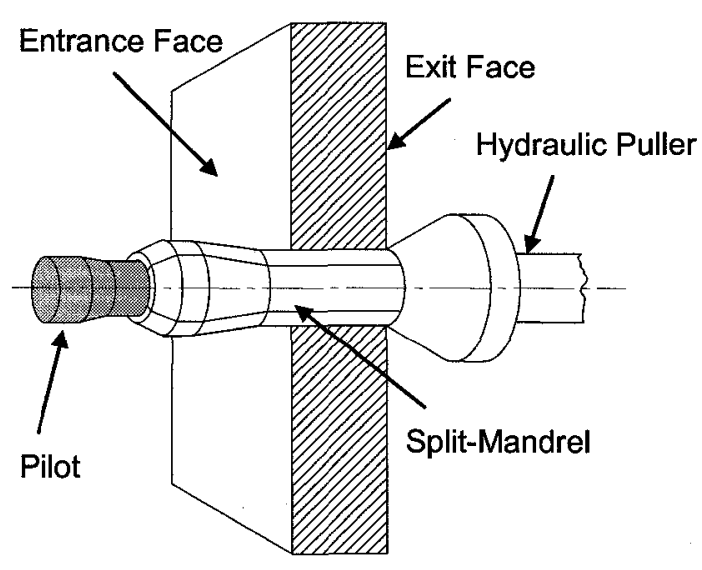

(a) split-mandrel

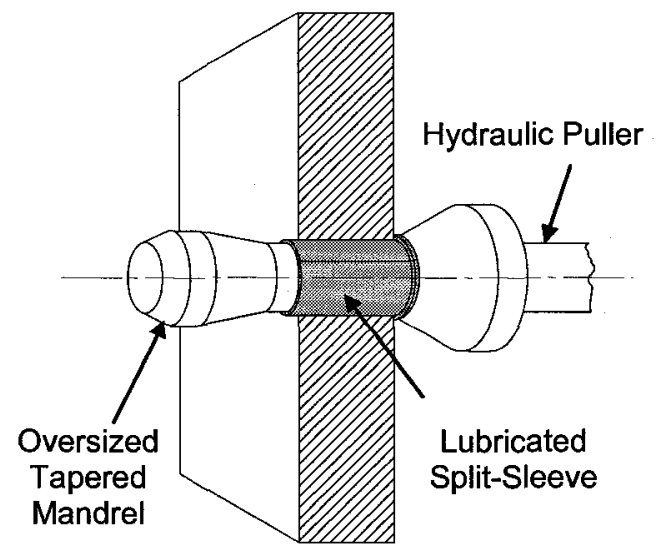

(b) split-sleeve

Figure 3.1: Schematic diagrams of split-mandrel and split-sleeve cold expansion processes 
hole. The combination of the mandrel major diameter and the sleeve thickness cold works the hole as the mandrel is drawn through the split-sleeve. The sleeve is then removed from the hole and discarded.

Both processes create axial ridges (or keys) as a result of opening of the slot(s) in the sleeve or mandrel during cold working (Figure 3.2). Each of the key locations represents a discontinuity in the residual stress field. These keys are removed during the final drilling/reaming step, although the effect on the residual stress state remains. Since the formation of these keys is unique to the cold expansion process, they do not relate to any mechanism in the rivet installation process.

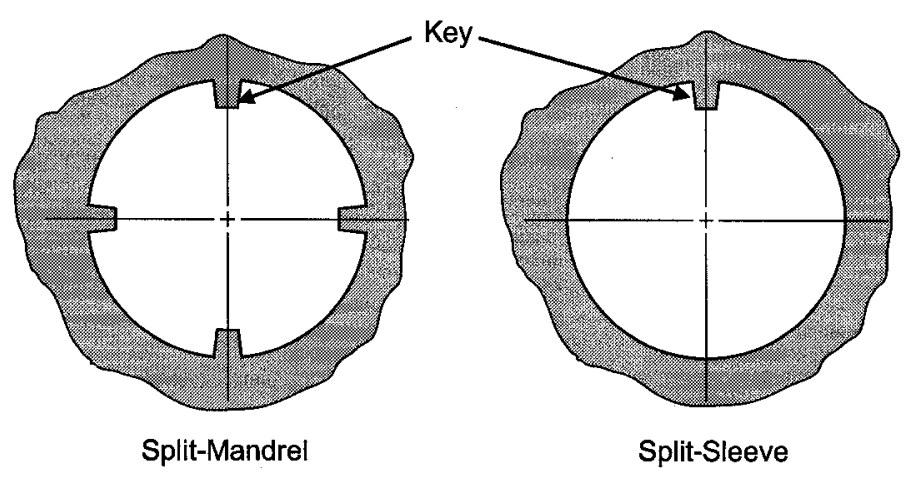

Figure 3.2: Illustration of key pattern for split-mandrel and split-sleeve cold expansion processes

Numerous studies have examined the formation of residual stresses and attempted to improve predictive capabilities. Analytical models based on the assumption of uniform expansion in an infinite sheet have been developed for both plane strain and plane stress conditions in monolithic materials [10-12]. The simplest of these analytical models for the case of a perfectly elastic-plastic material was presented earlier in Section 2.4.3. More recently, finite element methods have been used to investigate the effects of non-uniformities in practical cold expansion methods. Pavier et al. $[34,35]$ used a combination of 2- and 3-dimension finite element models to study the through-thickness residual stress distribution resulting from a split-sleeve cold expansion process. Results from this study showed that residual stresses along the entrance and exit faces of a cold expanded plate were substantially different from analytical model predictions. Although results between the FE 
and analytical models converged for locations away from the entrance and exit faces of the plate, neglecting the variation could result in non-conservative fatigue life predictions. Finite element techniques have also been applied in other studies to investigate different cold expansion techniques [30,31], the influence of adjacent holes [39], as well as other aspects of cold expansion $[37,38,40]$.

\subsection{Cold Expansion Finite Element Model}

As a precursor to modelling the the formation of residual stresses resulting from rivet installation, the split-sleeve cold expansion process was modelled. The development and implementation of this model is described in the remainder of this section.

\subsubsection{Model Description}

The explicit finite element code LS-DYNA v.970 [44] was used to analyze the split-sleeve cold expansion process (FTI process specification 8101D). For modelling purposes, the following simplifications to this process were made: the lubricated split-sleeve was removed and the mandrel was assumed to be rigid. To compensate for the lack of the split-sleeve, its thickness was added to the radius of the mandrel and a frictionless contact interface was used between the plate and the mandrel. Formation of a key as a result of the slot in the sleeve was also not considered. Additionally, the final reaming step to enlarge the cold expanded hole to nominal dimensions was not considered in this analysis.

A schematic of the FE model is shown in Figure 3.3, consisting of a rigid mandrel, rigid backing plate and deformable plate. The mandrel and backing plate were represented as rigid surface meshes while the plate consisted of eight-node single-point integration brick elements (ELFORM=1). Details regarding the mesh are given in Table 3.1 and Figure 3.4. The use of single-point integration brick elements introduces the potential for zero-energy deformation modes within the finite elements known as hourglass modes. These modes produce visible deformation without changing the element strain value computed at the integration point. 

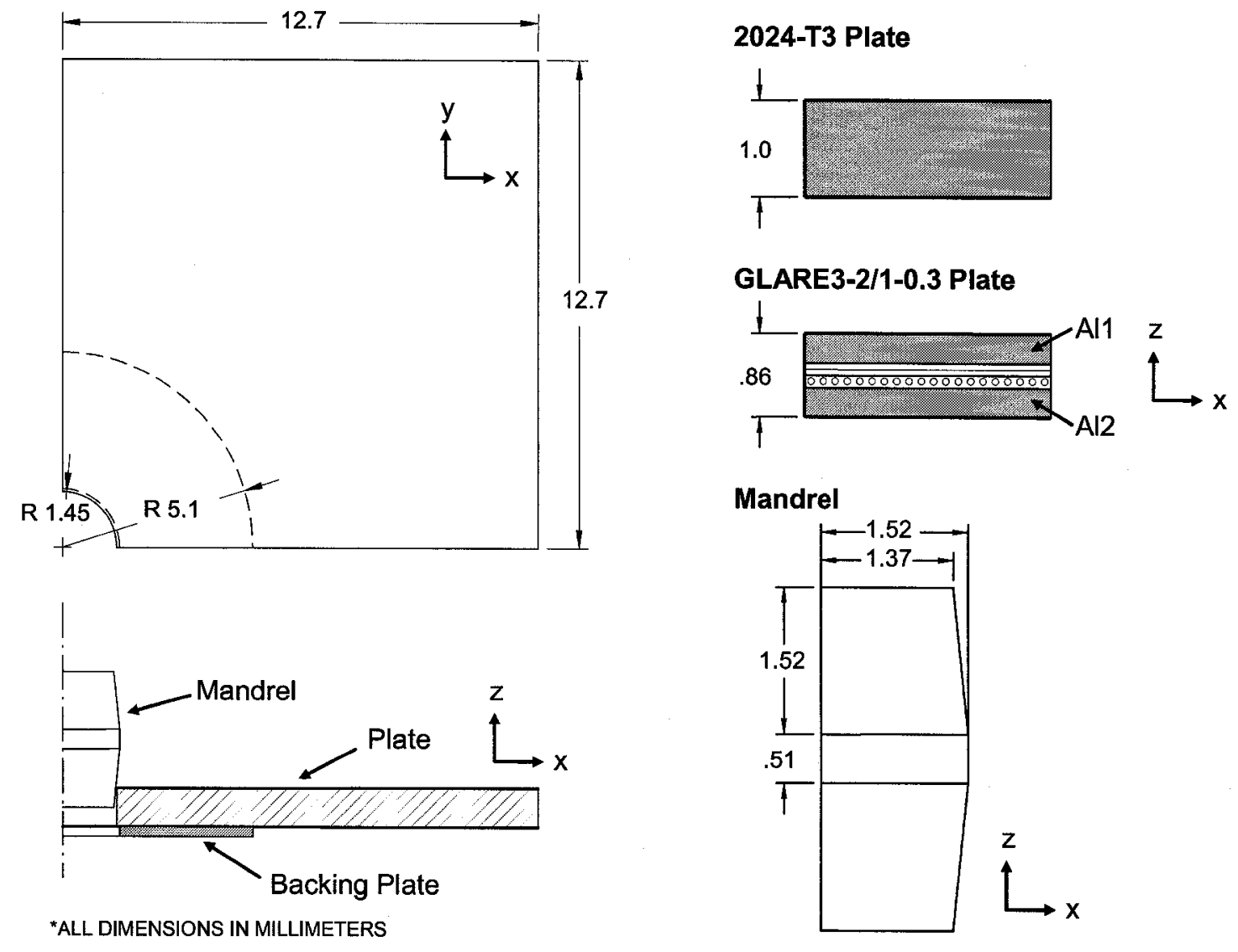

Figure 3.3: Schematic of cold expansion finite element model

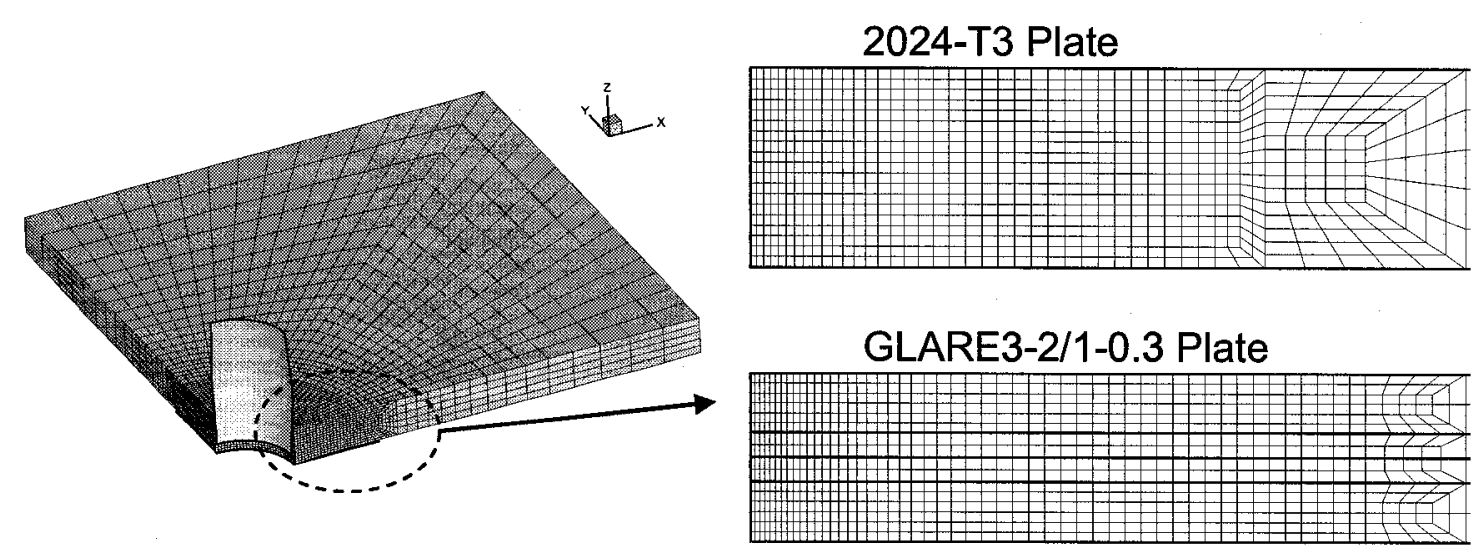

Figure 3.4: Finite element plate mesh 
Table 3.1: Cold expanded plate mesh details

\begin{tabular}{ccccc}
\hline Plate & $\begin{array}{c}\text { Total No. } \\
\text { Elements }\end{array}$ & $\begin{array}{c}\text { Through- } \\
\text { Thickness No. } \\
\text { Elements (hole } \\
\text { edge) }\end{array}$ & $\begin{array}{c}\text { Through- } \\
\text { Thickness No. } \\
\text { Elements } \\
\text { (boundary) }\end{array}$ & $\begin{array}{c}\text { No. Elements } \\
\text { along Hole } \\
\text { Perimeter }\end{array}$ \\
\hline 2024-T3 & 27990 & 19 & 5 & 30 \\
GLARE3-2/1-0.3 & 29820 & 16 & 6 & 30 \\
\hline
\end{tabular}

The use of higher-order elements avoids the potential for hourglassing; however, such elements are computationally more expensive and less suited to large-deformation problems due to their sensitivity to element distortion. To allow the use of single-point integration brick elements and avoid the occurrence of hourglass deformation modes, type- 6 stiffness based hourglass control options in LS-DYNA were employed. Time scaling was also invoked to reduce the computational time and take advantage of the low inertial effects (quasi-static nature) of the cold expansion process.

Quarter symmetry was utilized during the simulations with symmetry boundary conditions prescribed along the two symmetry planes and along the periphery of the plate. Axial motion of the plate was constrained through frictionless contact with the fixed backing plate and through nodal constraints applied to the exit-face nodes on the periphery of the plate. Motion of the mandrel was limited to the axis of the rivet hole (z-direction). The mandrel was translated through the plate at a constant feed rate starting from a position of initial contact with the plate until contact was broken. Subsequent to this, the backing plate was removed and the plate allowed to come to rest.

Contact was implemented into the simulations using segment-based automatic contact options in LS-DYNA [44]. Automatic contact allows the user to define contacting pairs by parts or groups of parts without the need for explicitly defining contact elements. LS-DYNA automatically generates contact elements as needed during the simulation in order to resolve contact between the defined contact pair. The current model included two contact pairs defining contact between the sheet and rigid mandrel, and between the sheet and rigid backing plate. Both of these interfaces were defined as frictionless. 
Simulations were performed for both 2024-T3 and GLARE3-2/1-0.3 plates with nominal thicknesses of $1.0 \mathrm{~mm}$ and $0.86 \mathrm{~mm}$ respectively. The hole radius $R$ was set to $1.45 \mathrm{~mm}$ corresponding to the FTI process specification for a $3.2 \mathrm{~mm}$ diameter fastener hole. Similarly, major and minor mandrel radii were set to $1.52 \mathrm{~mm}$ and $1.37 \mathrm{~mm}$ respectively. Plate dimensions of $25.4 \mathrm{~mm}$ long by $25.4 \mathrm{~mm}$ wide were used for both materials.

\subsubsection{Material Modelling}

Material models were required for both the 2024-T3 plate and the individual constituents of the GLARE3-2/1-0.3 laminate (2024-T3 aluminum plies, glass fibre-epoxy plies, and adhesive layers). In order to capture the plasticity of the aluminum plate and aluminum layers in GLARE, a non-linear power-law plasticity model with isotropic hardening was used (MAT 18). The appropriate constants for the model were obtained by curve fitting the stress-strain curve for 2024-T3 in the long transverse direction (grain direction) available in MIL-HDBK-5 [45]. Deformation of the fibre layers was assumed to be elastic, and an orthotropic elastic material model (MAT 2) using elastic constants based on experimental work by Hagenbeek [46] was used. A summary of the material properties used in these models is given in Table 3.2. The adhesive layers in the GLARE laminate were not represented with finite elements, and thus did not require a material model. Rather, the adhesive layer was accounted for using contact interfaces which is further described in the next section.

\subsubsection{Delamination Modelling for GLARE}

The presence of delamination damage after applying various cold expansion process to GLARE was noted in experimental investigations by van der Kuip [47]. Although the extent of these delaminations was typically below $20 \%$ of the original hole radius, they allow unrestrained elastic springback of the fibre layer in the delaminated area following cold expansion. Thus it was deemed important to include the influence of delaminations on the residual stress state in cold expanded GLARE.

Two possible cases were considered. In the first case (Case A), delamination was not considered and the interface between the various layers was defined as a tied contact interface. 
Table 3.2: Summary of FE material models $[45,46]$

\begin{tabular}{ll}
\hline Material Model Parameter & Value \\
\hline 2024-T3 (Power Law Plasticity) [45] & \\
Elastic modulus, $E$ & $72.4 \mathrm{GPa}$ \\
Strength coefficient, $k$ & $530 \mathrm{MPa}$ \\
Hardening exponent, $n$ & 0.10 \\
Poisson ratio, $\nu$ & 0.33 \\
Unidirectional Prepreg (Orthotropic Elastic) $[46]$ & \\
Local 11 elastic modulus, $E_{11}$ & $50.6 \mathrm{GPa}$ \\
Local 22 elastic modulus, $E_{22}$ & $9.9 \mathrm{GPa}$ \\
Local 33 elastic modulus, $E_{33}$ & $9.9 \mathrm{GPa}$ \\
Local 12 shear modulus, $G_{12}$ & $3.7 \mathrm{GPa}$ \\
Local 23 shear modulus, $G_{23}$ & $3.7 \mathrm{GPa}$ \\
Local 31 shear modulus, $G_{31}$ & $3.7 \mathrm{GPa}$ \\
Local 21 Poisson ratio, $\nu_{21}$ & 0.063 \\
Local 31 Poisson ratio, $\nu_{31}$ & 0.063 \\
Local 32 Poisson ratio, $\nu_{32}$ & 0.32 \\
\hline
\end{tabular}

${ }^{*} G_{23}$ was not provided in [46], so it was estimated to be equal to $G_{12}$ and $G_{31}$. Finite element results were found to be insensitive to variations of $\pm 10 \%$ in $G_{23}$.

This interface fixes nodes on adjacent faces of the interface together so that deformation of the interface is possible, but relative deformation at the interface faces is not. In the second case (Case B), delamination along the aluminum-prepreg interfaces was modelled using a tied interface with the addition of a shear stress and normal stress failure criterion known as a tiebreak contact interface [44]. This failure criterion is suitable for predicting delamination initiation but the rigid nature of the tied interface results in stress concentrations along the delamination front that cause over-predictions in delamination growth and size. To prevent such over-predictions, the extent of the delamination was limited by predefining an allowable delamination size with the tiebreak interface and surrounding it with a tied interface (Figure 3.5). Modelling delamination in this manner avoided the need to model the dynamics of delamination propagation and permitted the continued use of quasi-static assumptions in the simulations. Alternate interface failure models that included limited 


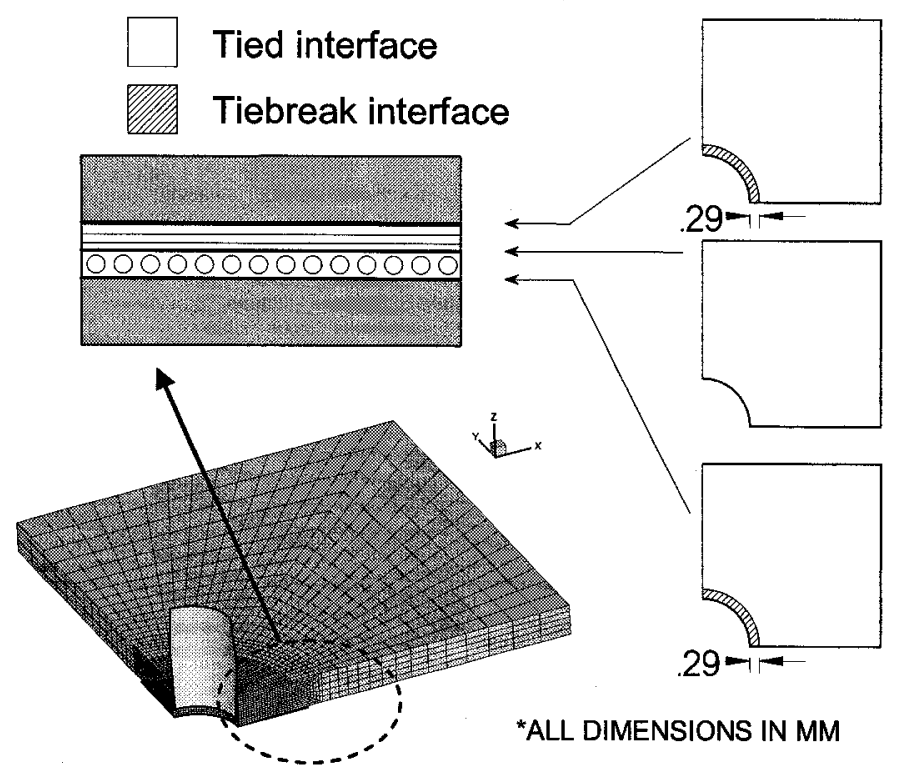

Figure 3.5: Illustration of contact interface definition between GLARE layers

plasticity of the interface were also attempted; however, requirements to accurately model delamination propagation conflicted with the mesh refinement requirements for accurate residual stress distributions, making computational times unfeasible.

Failure of the tiebreak interface was simplified in this application to neglect normal interface stresses, and a maximum shear stress of $43 \mathrm{MPa}$ was defined based on the maximum shear stress allowable for FM94 prepreg [24]. The predefined delamination size was set to $20 \%$ of the hole radius based on observations made by van der Kuip [47]. The same delamination size was defined for both aluminum-prepreg interfaces, and no delamination was permitted between the two prepreg layers. Subsequent to failure, the tiebreak interface acted as a standard surface-to-surface contact interface.

\subsubsection{Model Verification}

Sensitivity studies on mesh size, simulation time, and other simulation parameters was performed to ensure accuracy in the analysis results. For conciseness, only results from the refined finite element model are presented here. Details and results from the refinement process are described in Appendix A. 


\subsection{Finite Element Results}

Residual radial and tangential stress distributions for 2024-T3 and GLARE3-2/1-0.3 plate were obtained from the finite element simulations. For presentation purposes, stress results are normalized using the yield stress $\sigma_{y}(290 \mathrm{MPa})$ of the 2024-T3 aluminum plate/GLARE layers. For brevity, results for the GLARE simulations are presented along the xz-plane only (Figure 3.3) unless otherwise stated.

\subsubsection{4-T3 Residual Stress Distribution}

The residual radial and tangential stress distributions observed for the 2024-T3 finite element simulations are illustrated in Figure 3.6 and Figure 3.7 respectively. Both figures illustrate the degree of through-thickness variation in the residual stress field that occurs as a result of non-uniformities in the simulated cold expansion process. A major limitation of existing analytical models is the inability to account for such non-uniformities. Previous finite element studies were found in the literature which also examined the through-thickness variation in residual stress $[34,35,40]$. Although none of these studies examined the same plate, hole, and mandrel geometries, the residual stress trends and magnitudes agreed well with results from the present investigation.

Mid-plane results from the 2024-T3 simulation are compared to various analytical models in Figure 3.8. Despite the non-uniformities present in the cold expansion process, the analytical models for uniform expansion accurately predict the residual stress distribution away from the exit and entrance faces of the sheet. Practically speaking, the exit and entrance faces are of concern for fatigue as they are the likely crack nucleation sites due to secondary bending stresses and fretting damage. However, the non-uniformities that arise during cold-expansion are different from those that arise during riveting and will not be a focus for discussion here. 


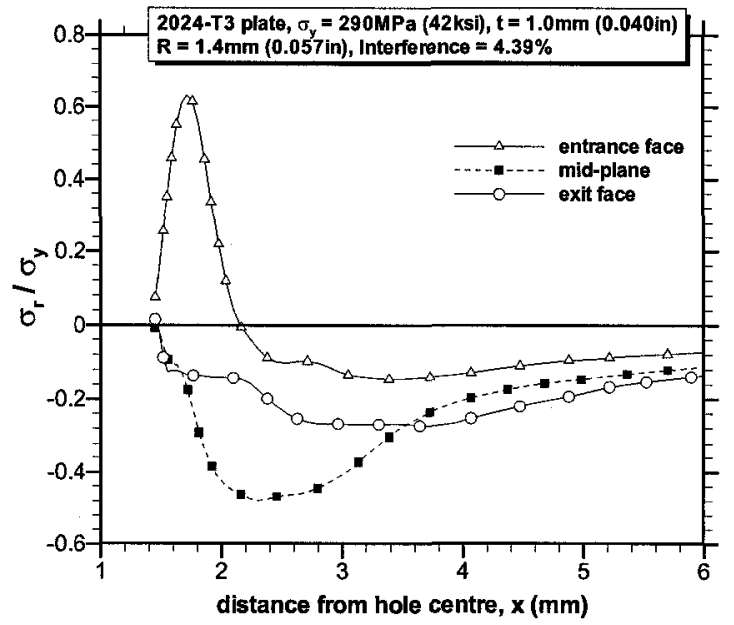

(a) 2-D line

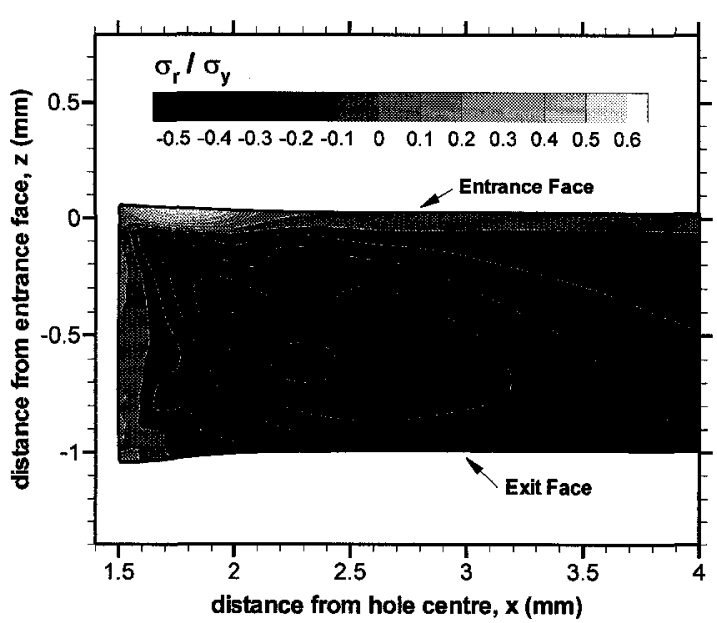

(b) 2-D contour

Figure 3.6: FE residual radial stress distribution for cold expanded 2024-T3 plate

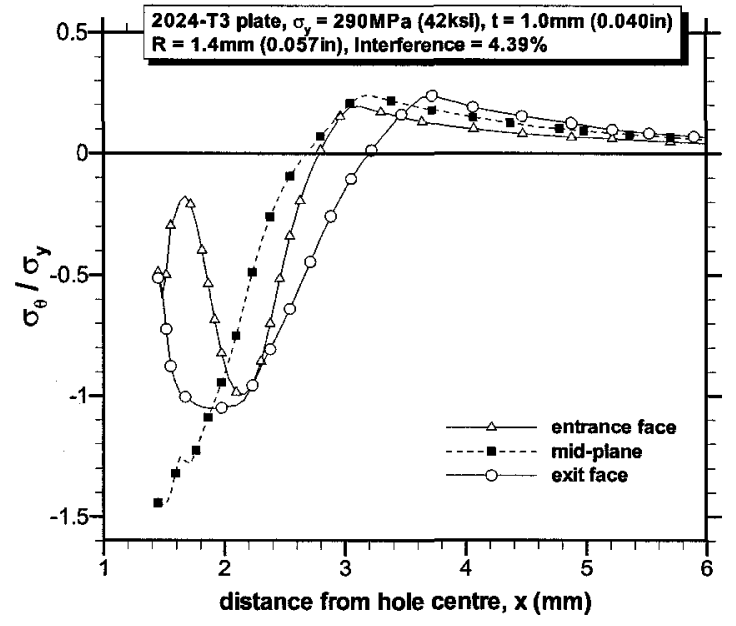

(a) 2-D line

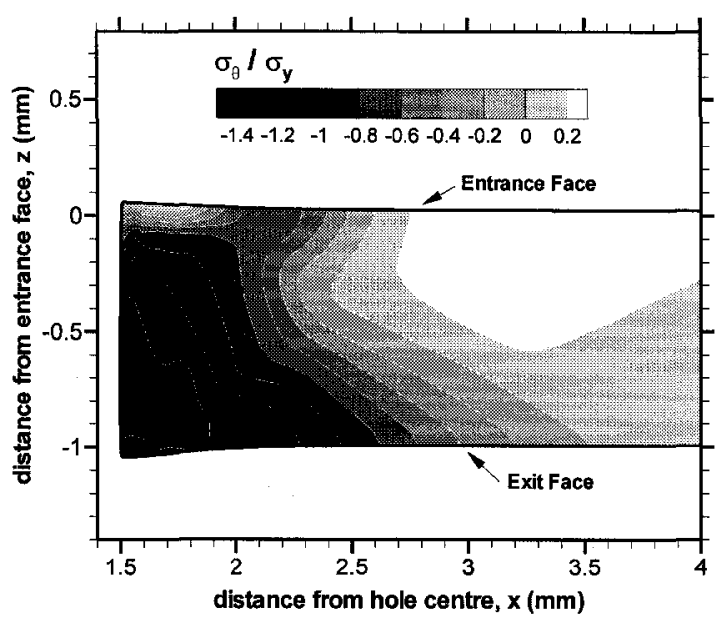

(b) 2-D contour

Figure 3.7: FE residual tangential stress distribution for cold expanded 2024-T3 plate 


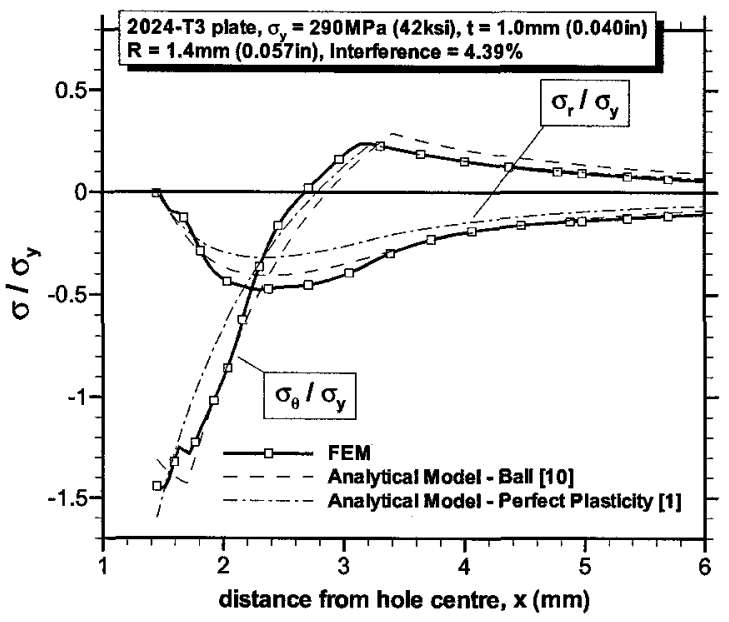

Figure 3.8: Comparison of 2024-T3 FE mid-plane residual stress results to analytical models

\subsubsection{GLARE3-2/1-0.3 Residual Stress Distribution}

The residual stress distributions obtained from the GLARE simulations are given in Figures 3.9 to 3.11. These results exhibit similar trends as the 2024-T3 simulations; however, a larger radius of plastic deformation indicated by the location of the peak in tensile tangential

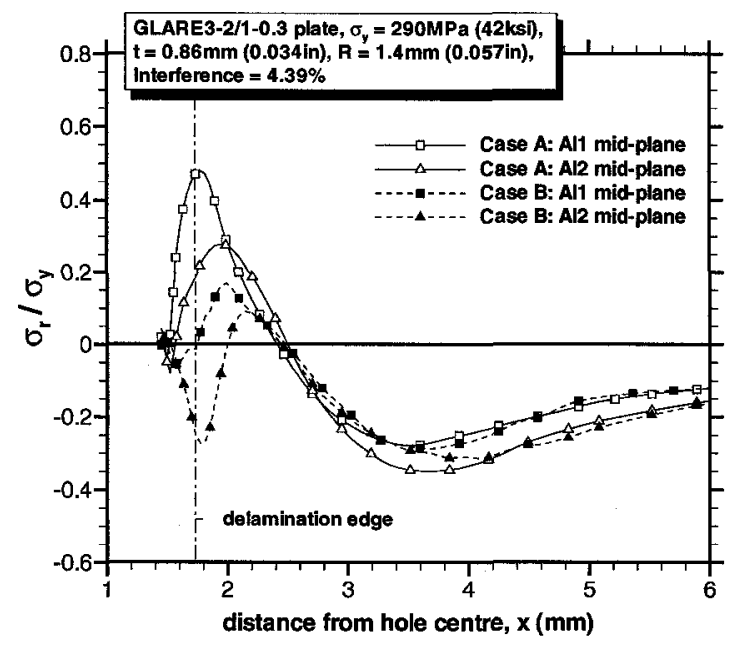

(a) radial stress

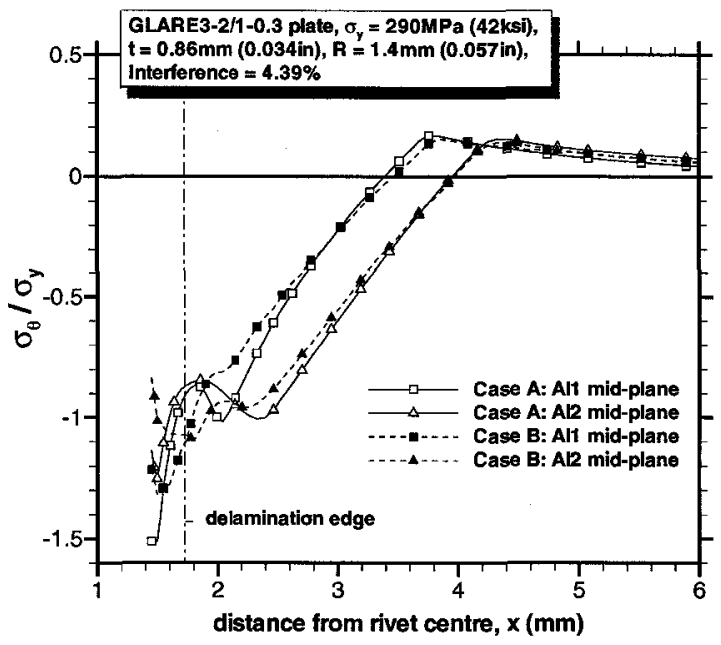

(b) tangential stress

Figure 3.9: FE mid-plane residual stress distributions for cold expanded GLARE3-2/1-0.3 plate 


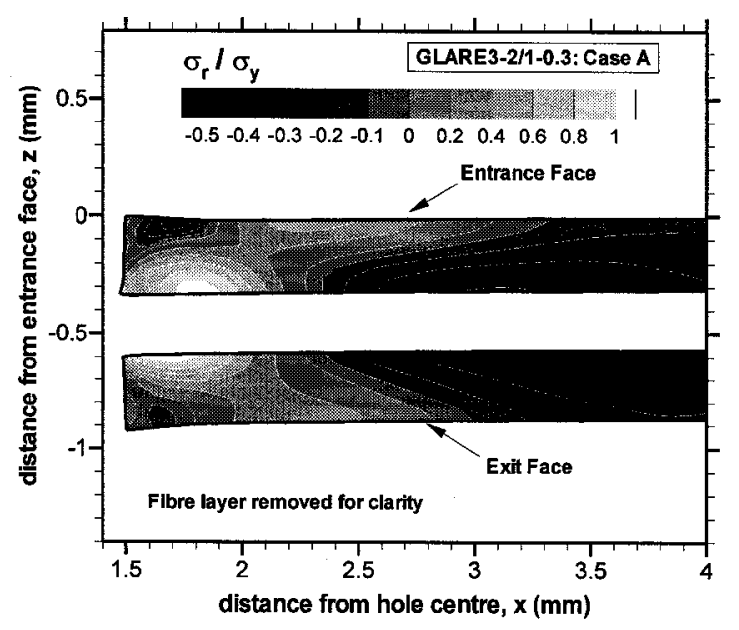

(a) no delamination

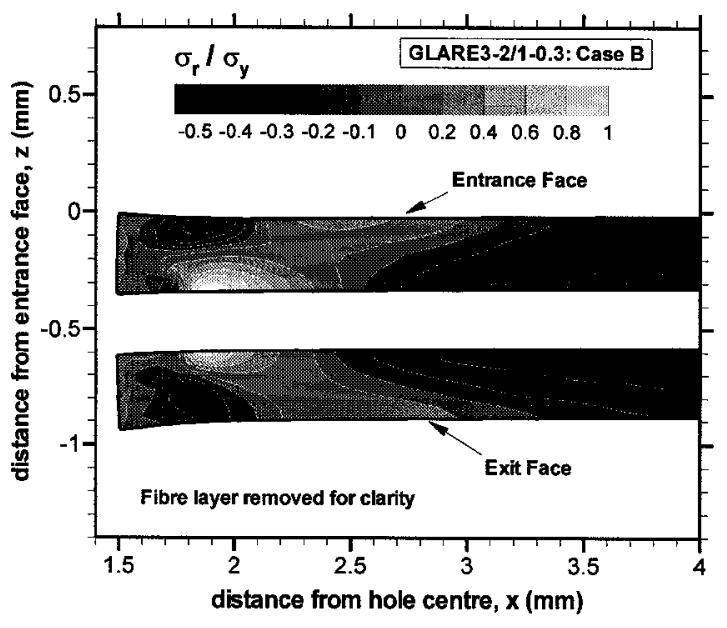

(b) limited delamination

Figure 3.10: FE residual radial stress contours in GLARE for different delamination models

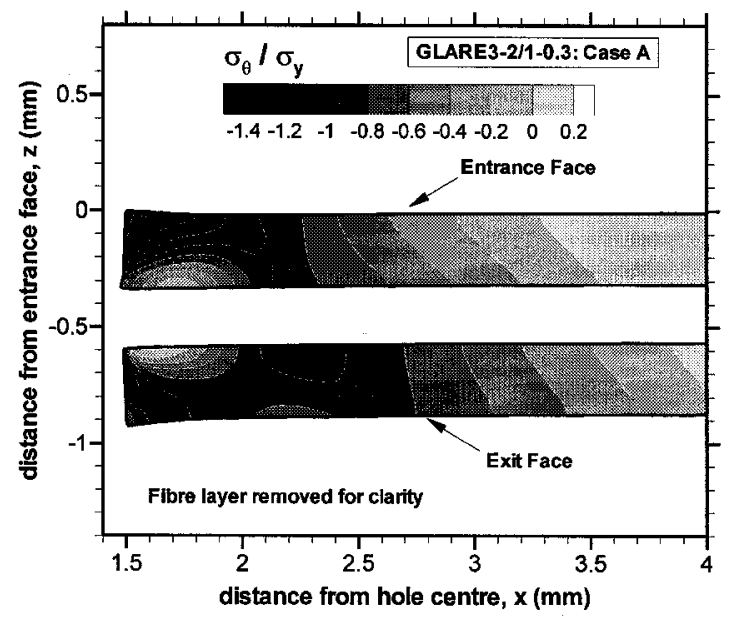

(a) no delamination

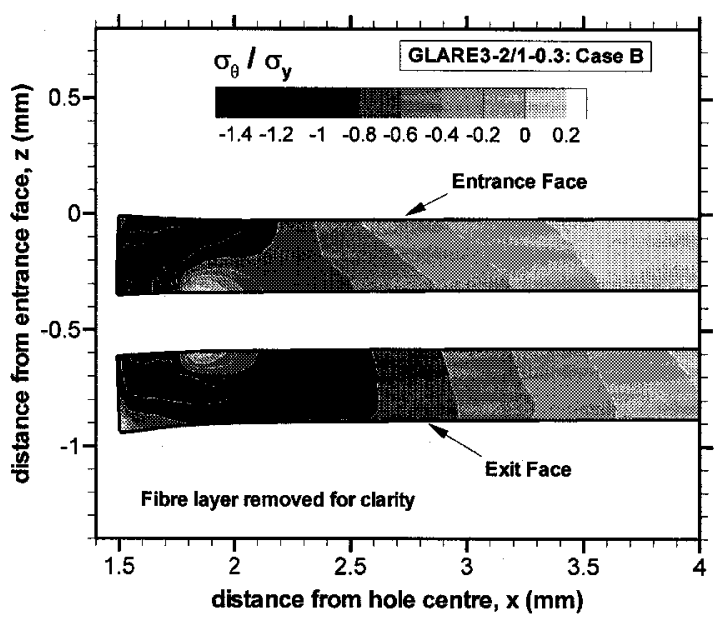

(b) limited delamination

Figure 3.11: FE residual tangential stress contours in GLARE for different delamination models 
stress is clearly evident in GLARE. This behaviour can be attributed to the apparent strain hardening behaviour of the fibre layers. As the aluminum layers begin to yield, their stiffness drops and load is redistributed through the elastic fibre layers, producing a stiffer yield response than monolithic aluminum (Figure 3.12). In a strain driven process such as cold expansion, this results in a larger applied load producing the larger region of plastic flow. This dependence on strain hardening is also predicted by analytical models [10].

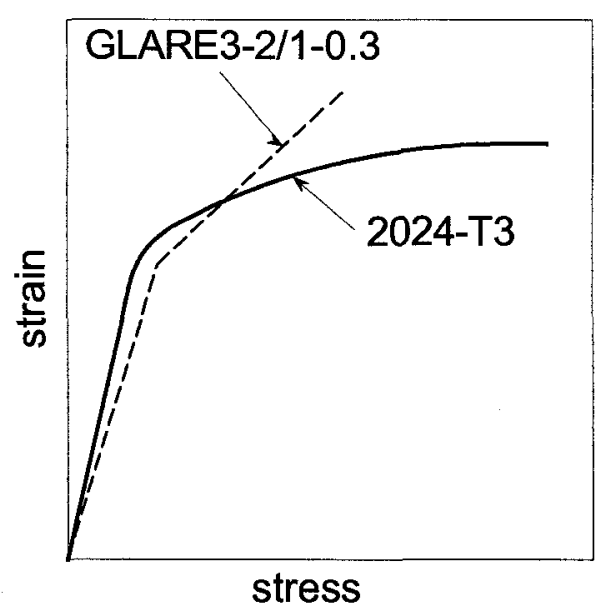

Figure 3.12: Schematic of 2024-T3 and GLARE stress-strain curves

Variations in the radius of plastic deformation at the mid-plane are also evident in Figure 3.9. As the cold-expansion process is not achieved uniformly though the thickness, the through-thickness anisotropy of the GLARE laminate affects the expansion of the entrance face aluminum layer (Al1) and exit face aluminum layer (Al2). As the mandrel is pulled through the laminate, it first encounters and expands Al1. Due to the plasticity of All, this initial expansion is achieved through localized plastic deformation near the hole edge. As the mandrel travels further through the laminate, elastic expansion of the fibre layer further expands Al1 and begins to expand Al2 through shear. This shear driven expansion results in more uniform expansion of the aluminum layers and increases the radius of the yield zone as described above. Once the mandrel reaches Al2, this layer has been nearly fully expanded through shear stresses from the fibre layer, and does not receive the same localized plastic deformation near the hole edge as does Al1. The shear dominated expansion of Al2 results 
in a larger yield zone but generates smaller residual compressive tangential stresses near the hole edge (Figure 3.13) due to the lower degree of plastic deformation in this region compared to All.

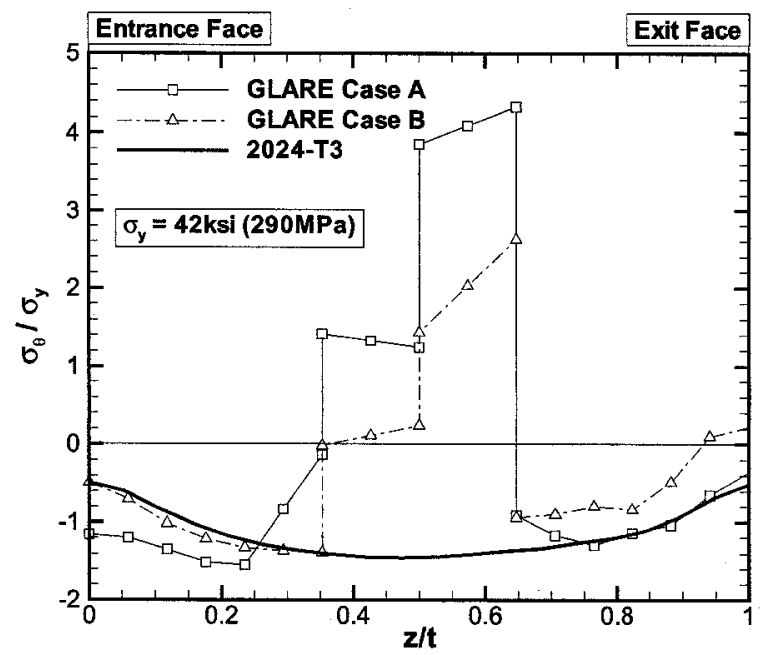

Figure 3.13: Residual tangential stress variation through plate thickness at hole edge

The influence of in-plane anisotropy of the GLARE laminate on the residual stress distribution is shown in Figure 3.14. Results along the primary material axes are nearly identical, indicating that the residual stresses are more strongly dependant on the overall fibre layer (the $0^{\circ}$ and $90^{\circ}$ unidirectional cross-ply layer) rather than the adjacent unidirectional prepreg layer. Results along the $\theta=45^{\circ}$ axis differ due to the lower stiffness of the fibre layer. Most notably, the radius of the plastic zone decreases as a result of a reduced influence of the fibre layer on the shear driven expansion.

\subsubsection{Influence of Delamination Model}

In the GLARE simulations not including the delamination model (Case A), the interface between the fibre and aluminum layers remains intact causing the aluminum layers to resist the elastic springback of the fibre layer. This resistance results in further contraction of the aluminum layers and increased residual compressive tangential stresses at the hole edge. Away from the free edge of the hole, large tensile radial stresses develop, reducing the compressive tangential stresses creating the clear local stress peak visible in Figure $3.9 \mathrm{~b}$. 


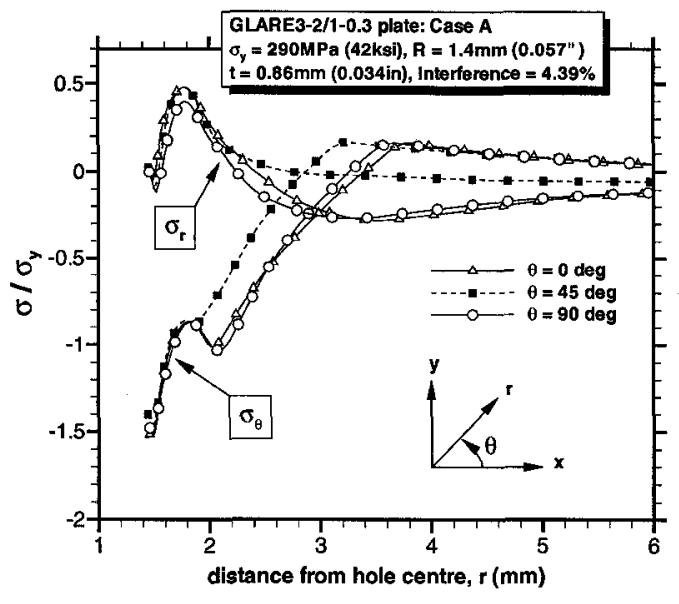

(a) no delamination

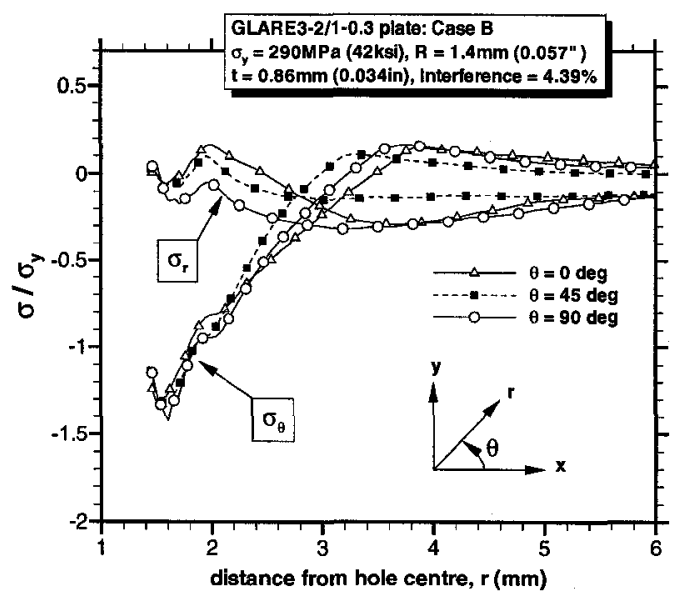

(b) limited delamination

Figure 3.14: Effect of fibre layer anisotropy on mid-plane residual stress distributions in GLARE

Introduction of the delamination model allows unrestrained springback of the delaminated portion of the fibre layer, thus reducing the radial tensile stresses (and associated strain energy) within the aluminum layers. Similarly, a reduction in the compressive tangential stresses at the hole edge also occurs.

Although delamination damage is often regarded as undesirable, it is a critical element of the damage tolerant behaviour of GLARE. The formation and growth of a delamination between a cracked aluminum layer and adjacent fibre layer increases the length over which the fibre layer is elongated due to crack opening displacements. As a result, overstraining is prevented and the fibre layer remains intact. The growth of this delamination is balanced by a reduction in interface shear which also results from the reduction in local strain of the fibre layer.

Similarly, the formation of a delamination due to cold expansion is also beneficial; however in this case the driving force is the cold expansion displacement (or more accurately, the retained expansion of the aluminum layers) rather than crack opening displacement. Reductions in residual stresses in the fibre layer and interface shear stresses between the fibre and aluminum layers (Figure 3.13) will help preserve the integrity of the fibre layer and reduce the driving force for further delamination growth. Furthermore, the aluminum 
layers also benefit from the delamination through a reduction in residual tensile radial stress which could facilitate fatigue crack initiation (Figure 3.9a).

In discussing the importance of delamination damage to the damage tolerance of GLARE, a caveat regarding balanced delamination formation and growth must be made. The above described benefits only occur when the size and growth of a delamination are comparable to the fatigue damage within the adjacent aluminum layers. If a delamination grows too large relative to its corresponding crack, crack opening is not restrained, negating the benefits of the fibre bridging mechanism. This has been observed in previous studies [48] on the fatigue performance of GLARE lap joints. In this study the potential benefits of dimpling, an alternative countersink method involving plastically deforming the sheet material in a set of die tools, were investigated. This process was found to introduce large delaminations which limited the effectiveness of fibre bridging, drastically reducing the expected damage tolerance of the GLARE sheet. Thus, although delamination is an integral component of the damage tolerance of GLARE, it does not mean that all delamination damage is beneficial.

The results of this cold expansion finite element study need to be viewed within the limitations of the delamination model that was used. Delamination was modelled as an initiation event with predefined delamination sizes. As a result, the influences of delamination propagation and size are not present in the results. Furthermore, the tied and tiebreak interfaces used to model adhesion between the aluminum and prepreg layers were rigid contact interfaces. As a result, stress concentrations that would be reduced by limited plasticity of the adhesive layer were generated along the edge of the delamination. For this reason, results for the GLARE simulations were presented along the mid-plane of the aluminum layers away from the stress concentration at the adhesive interface.

\subsection{Conclusions}

The present study was undertaken as a precursor to simulating the rivet installation process, providing a means to verify the finite element techniques being used. A 3D finite element 
analysis was carried out to investigate the formation of residual stresses due to the splitsleeve cold expansion process. The formation of residual stresses in monolithic 2024-T3 aluminum and GLARE3-2/1-0.3 plate were considered. Based on the results of the finite element analysis, the following conclusions can be made:

- Agreement between the 2024-T3 plate results, analytical models, and existing finite element studies indicate that the material models and finite element techniques employed in this study are acceptable for predicting the formation of residual stresses in monolithic 2024-T3.

- In contrast to monolithic 2024-T3, expansion of the aluminum layers in GLARE occurs by two mechanisms: direct expansion through radial pressure between the aluminum layer and mandrel, and shear driven expansion resulting from the elastic expansion of the fibre layer.

- Cold expansion of GLARE results in a larger region of plastic flow compared to monolithic 2024-T3 plate. This is attributed to the apparent strain hardening behaviour produced by the redistribution of load by the fibre layer during yielding conditions.

- Formation of a delamination during cold expansion permits unrestrained elastic springback of the delaminated portion of the fibre layer. The dissipation of elastic strain energy in the fibre layer is complemented in the aluminum layer by a reduction in tensile radial stresses and compressive tangential stresses near the hole edge. The reduction in residual stress within the fibre layer serves to protect its integrity under superimposed loading in a manner analogous to the delaminations which form during the fibre bridging mechanism.

Overall, the results from this study increase the level of confidence in the finite element techniques used and to be used in subsequent studies. Additionally, the observations made for GLARE will provide a basis for interpreting results for the formation of residual stresses during rivet installation, which is the topic of the next chapter. 


\section{Chapter 4}

\section{Riveting Induced Residual Stresses}

\subsection{Introduction}

Although analogous to the cold expansion process, the formation of residual stresses during rivet installation is inherently more complex. The degree of hole expansion varies through the sheet thickness as a result of non-uniform rivet expansion. During installation, both rivet heads constrain the sheets, introducing a through-thickness pressure component which contributes to yielding in the sheet. Subsequent to installation, the rivet limits the amount of elastic springback in the sheets. These factors combined with additional riveting variables such as rivet type and squeeze force result in a complex residual stress state that cannot be easily predicted using existing analytical models developed for cold expansion processes.

This chapter presents a finite element study undertaken to investigate the formation of residual stress due to rivet installation. First, a brief review of riveting practices is presented based on the current understanding of the formation of residual stresses in rivet joints and previous studies on rivet installation. The results of the present finite element investigation are then discussed in relation to this current understanding. As in the previous chapter, the finite element study was completed for both monolithic 2024-T3 and GLARE3-2/1-0.3 sheet materials in order to investigate potential differences in the formation of residual stresses in the two sheet materials. 


\subsection{Review of Riveting Induced Residual Stresses}

Several beliefs have been established in the design community regarding the formation of residual stresses due to rivet installation, inferred primarily from overall fatigue life data obtained both experimentally and from in-service components. Directly observing the influence of residual stresses on crack nucleation and growth is generally not possible as the residual stress field is localized in the region hidden by the the rivet heads.

One such belief has been based on the observation that the use of universal rivets results in an improvement in overall fatigue performance over a similar joint with countersunk rivets. It is believed that the conical hole surface in the outer sheet of a countersunk joint is less effectively expanded than the cylindrical hole surface present in a universal rivet joint. Relating to the cold expansion analogy, this would result in a more favourable residual stress state in the outer sheet of a universal joint.

This importance placed on the cylindrical section have also led to several sets of guidelines regarding the installation of countersunk rivets. Countersink depths are often limited to ensure that a portion of the countersunk hole remains cylindrical. Müller [1] reported a range of 0.1 to $0.2 \mathrm{~mm}$ based on the requirements used by several aircraft manufactures including BAe, Fokker, and MBB Aerospace. These guidelines also ensures that a through-countersink is avoided, which introduces an additional stress concentration due to the presence of a knife edge.

Countersunk depths are also often limited to ensure a small extension of the countersunk rivet head above the joint surface before installation. This extension is believed to improve

hole filling and expansion of the countersunk sheet. Again, Müller [1] reported a range of 0.01 to $0.2 \mathrm{~mm}$ based on the requirements used by several aircraft manufactures. Some rivet designs also incorporate a deformable spherical rivet crown, which reduces in height during installation to achieve the above effect while minimizing the final extension of the installed rivet.

Arguably the most significant contribution to understanding and exploiting the formation of residual stresses is the concept of using the rivet squeeze force $\left(F_{S q}\right)$ as a measure of 
installation quality. The benefits of force controlled riveting were extensively studied, both experimentally and analytically, by Müller [1]. Through his work, Müller indeed verified that squeeze force is a more relevant quality control measure than driven rivet geometry. Driven rivet geometry was dependent on many additional factors, including rivet length, and did not provide as accurate a measure of the degree of hole expansion. In addition to his examination of the rivet squeeze force, Müller provided a detailed review of the contributing factors to the fatigue performance of riveted lap joint which has formed the basis for additional studies.

Subsequent to Müller's work, several finite element studies have been undertaken to further understand the relationship between squeeze force and residual stress. Recognizing the lack of non-linear stress-strain data available for the 2117-T4 aluminum alloy commonly used for rivets, Szolwinski [6,7] conducted compression tests on billets machined from actual rivets. Szolwinski incorporated this data into an axi-symmetric finite element model which was used to study the installation of universal rivets in 2024-T3 aluminum plate. Li et $a l$. $[15,16]$ undertook the task of providing experimental data to verify results being obtained using finite element techniques. Conventional stress measurement techniques do not provide adequate results for verification as the primary regions of interest lie hidden beneath the rivet head. To overcome this, $\mathrm{Li}$ et al. used neutron diffraction techniques to obtain results beneath the rivet head, providing data which reinforced their own and previous finite element studies. Ryan and Monaghan [17] investigated the formation of residual stresses in GLARE laminates using a simplified axi-symmetric model. Use of an axi-symmetric model, however, prevented the orthotropic behaviour of the fibre layers from being fully captured thus limiting the results to a qualitative nature. Other studies have investigated additional structural design aspects such as fastener clamping and crashworthiness [13,14,49], however, these studies are beyond the scope of the present investigation.

The current understanding of riveting induced residual stresses comprises of several experience based rules of thumb and the identification of the rivet squeeze force as a more suitable measure of rivet installation quality. The present investigation will expand on this knowledge base by simulating a force controlled riveting process using 3-dimensional finite 
element methods. Force controlled riveting will be examined from the standpoint of these design rules of thumb, attempting to identify key mechanisms responsible for the formation of residual stresses.

\subsection{Rivet Installation Finite Element Model}

In order to study the formation of residual stresses during riveting, the finite element model presented in Chapter 3 was extended to simulate the riveting process. The development and implementation of this model is described in the remainder of this section.

\subsubsection{Model Description}

The configuration chosen for the model consisted of two plates joined at their centres by a single $3.2 \mathrm{~mm}$ diameter 2117-T4 aluminum rivet. Both 2024-T3 and GLARE3-2/1-0.3 plate with thicknesses of $1.0 \mathrm{~mm}$ and $0.86 \mathrm{~mm}$ respectively were simulated. Installation of universal head (U.S. military specification MS20470AD4-4) and reduced-depth countersunk head (NAS1097AD4-4) rivets of length $6.4 \mathrm{~mm}$ were investigated. The model configuration is shown schematically in Figure 4.1.

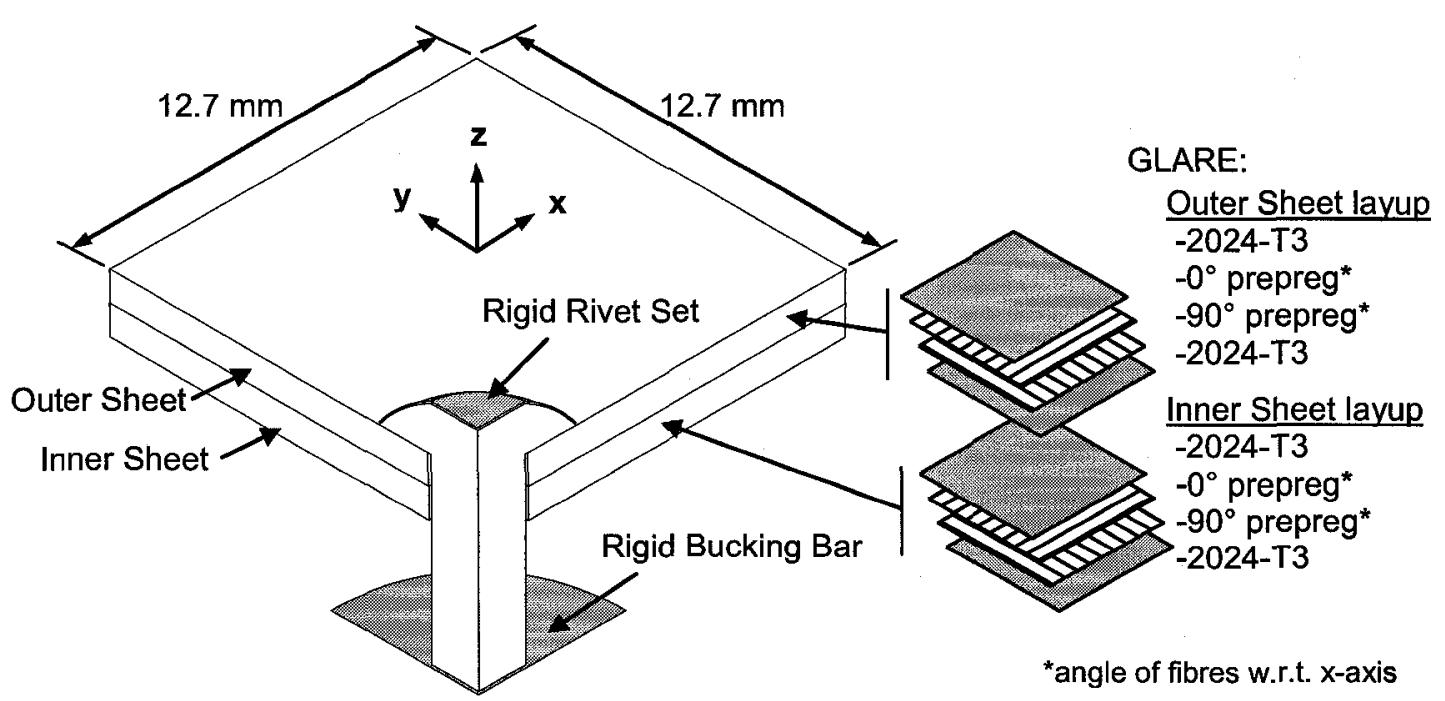

Figure 4.1: Schematic of rivet installation finite element model 

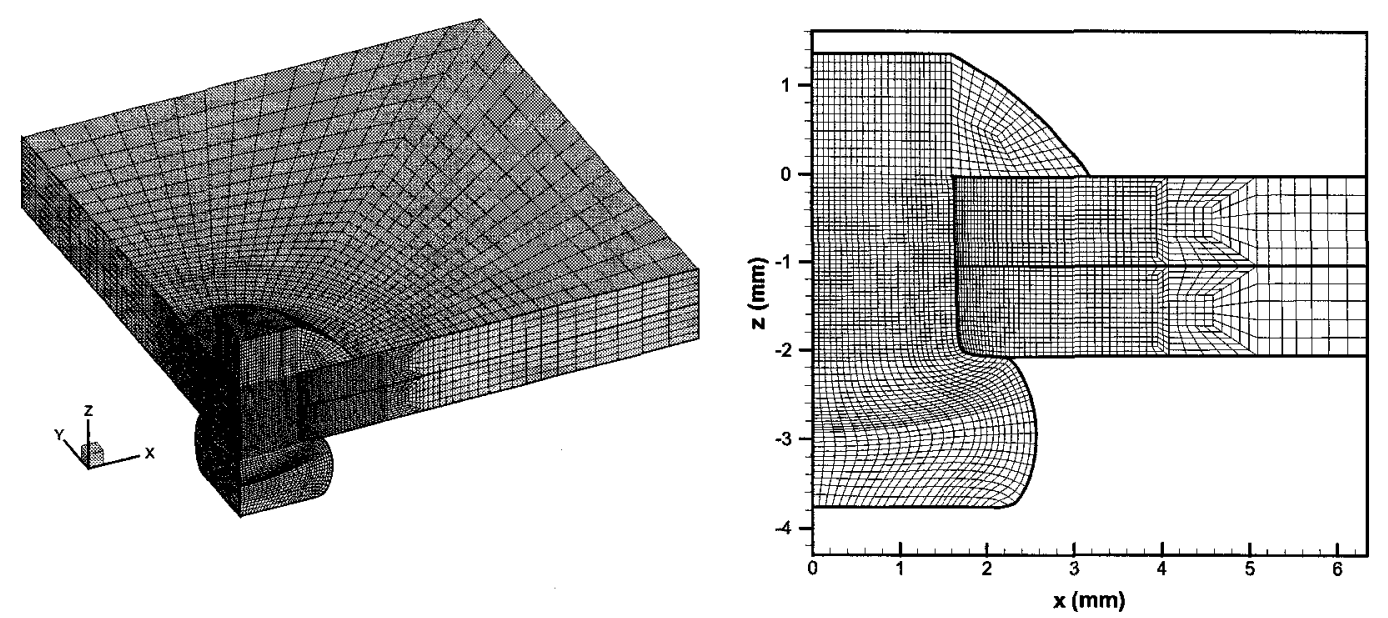

Figure 4.2: Typical mesh density shown for deformed universal rivet in 2024-T3 plate

A typical mesh for the universal head rivet case is shown in Figure 4.2. 8-node singlepoint integration brick elements (ELFORM=1) were used to represent the rivet and plates. Riveting tools were defined as rigid surfaces using 4-node shell elements. As described in Chapter 3, the use of single-point integration brick elements introduces the potential for hourglass deformation modes. To allow the use of single-point integration brick elements and avoid the occurrence of hourglass deformation modes, type- 6 stiffness based hourglass control options in LS-DYNA were employed.

Symmetry boundary conditions were applied along the two quarter symmetry and two plate periphery planes. The outer and inner plates were further constrained by restricting motion of the nodes along the periphery of their free surface in the rivet axis direction. Motion of the rigid riveting tools was constrained by fixing the position of the rivet set and restricting the rivet bucking bar to translation along the rivet axis (Figure 4.3). All other constraints were provided through contact definitions specified using the segmentbased automatic contact options in LS-DYNA between contact pairs (automatic contact was discussed in Chapter 3). The current model included four contact pairs defining contact between the rivet and each of the rivet tools, between the rivet and plates, and along the faying surface between the inner and outer plate. A coefficient of friction of 0.18 , obtained experimentally by Ryan [17], was specified for each of the contact interfaces. 


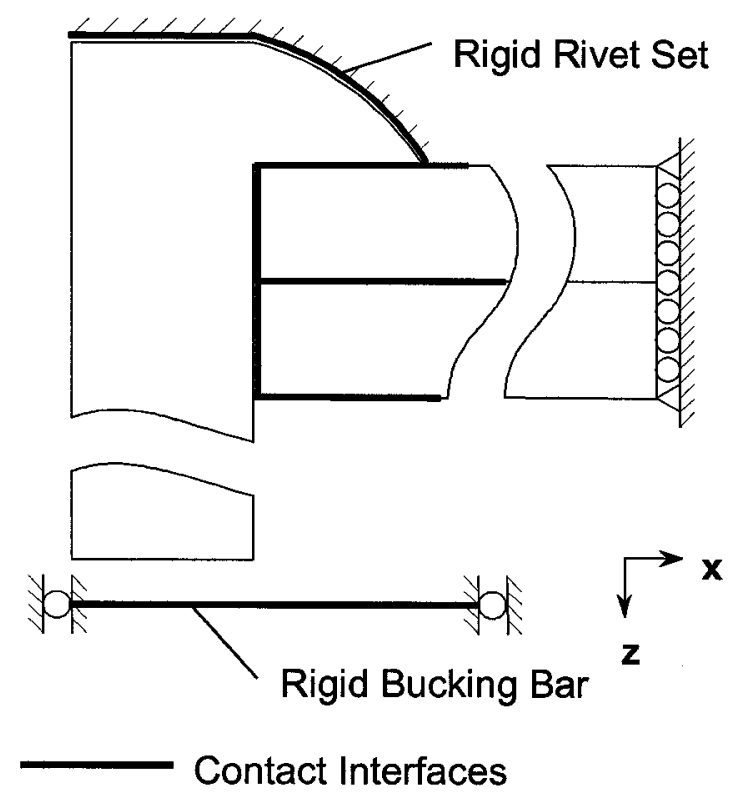

Figure 4.3: 2-D schematic of FE boundary conditions

Installation of the rivet was simulated by applying a ramp load-unload cycle to the rigid bucking bar. Due to the nature of explicit time integration schemes, two dwell periods were included after the loading and unloading phases to allow the system to fully come to rest. A residual squeeze force of $2 \mathrm{~N}$ was prescribed at the end of the unloading phase in order to prevent oscillations in the simulation from forming due to a break in contact between the rivet and riveting tools, thereby minimizing the required dwell time. Five squeeze forces were considered in this study: $1000,1500,2000,2500$, and $3000 \mathrm{lbf}(4.4,6.8,8.9,11.1$, and $13.3 \mathrm{kN}$ respectively). These squeeze forces result in approximate $D / D_{0}$ ratios of $1.2,1.4$, $1.5,1.6$, and 1.7 respectively. Quasi-static assumptions were applied and simulation time was scaled to minimize processing time.

\subsubsection{Rivet Geometries}

Three rivet geometries were used in this investigation and are summarized in Figure 4.4. The first geometry was used to represent the MS20470AD4-4 rivet case considered in this study. Minor simplifications to the rivet geometry were made for modelling purposes as shown in 

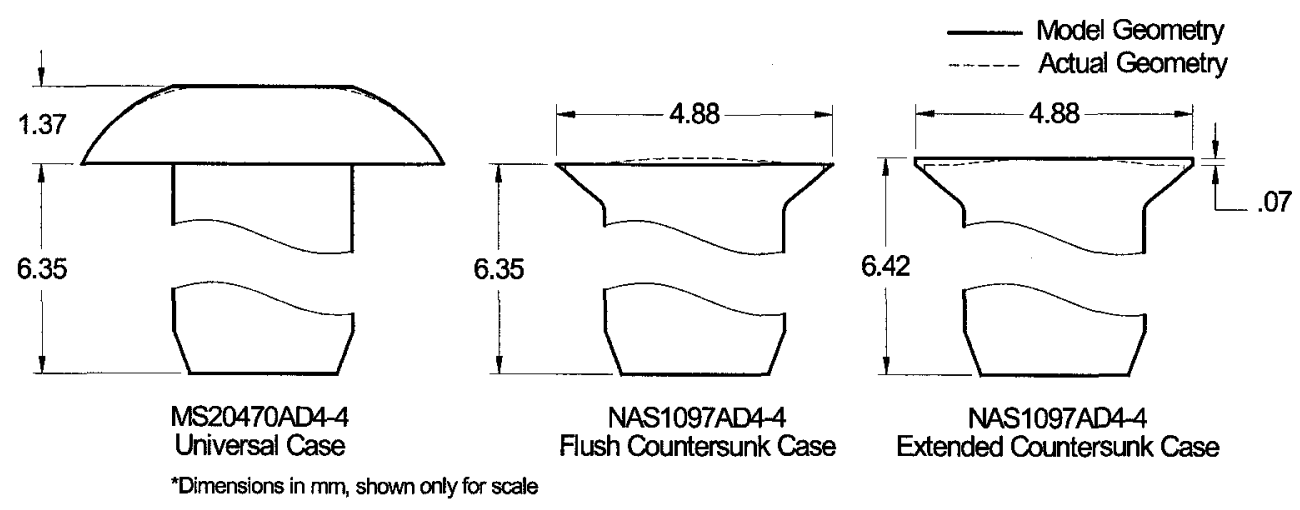

Figure 4.4: Comparison of FE model and actual rivet geometries

Figure 4.4. The second and third geometry were used to represent the NAS1097AD4-4 rivet case. Again, minor simplifications were made to the rivet geometry for modelling purposes. Two rivet geometries were used for this case in order to study the effect of a small extension of the countersunk rivet head above the joint surface during riveting. The second geometry in Figure 4.4 was used to simulate the perfectly flush case. The third geometry included a $0.07 \mathrm{~mm}$ crown on the rivet head for simulating the case of a $0.07 \mathrm{~mm}$ extension. A value of $0.07 \mathrm{~mm}$ was chosen as it corresponds to the nominal crown height on a NAS1097AD4-4 rivet as well as the extension height studied by Müller [1]. Extension of the rivet head was implemented through the addition of a crown to allow the same countersunk sheet geometry to be used for both countersunk rivet geometries. Additionally, this implementation ensures that the countersink depths are identical for the two cases and isolates any effects that may arise due to differences in the height of the cylindrical portion of the countersunk hole.

\subsubsection{Material Modelling}

Non-linear material models were required for the 2024-T3 and GLARE3-2/1-0.3 plate materials as well as the 2117-T4 rivet material. The material models used for the two plate materials were previously described in Section 3.3.2. Despite the common use of 2117-T4 as a riveting material, relatively few studies into the large strain behaviour of 2117-T4 have been conducted. Review of the literature found two separate studies which resulted in suitable non-linear material models. The first model, developed by Szolwinski and Farris [7], 


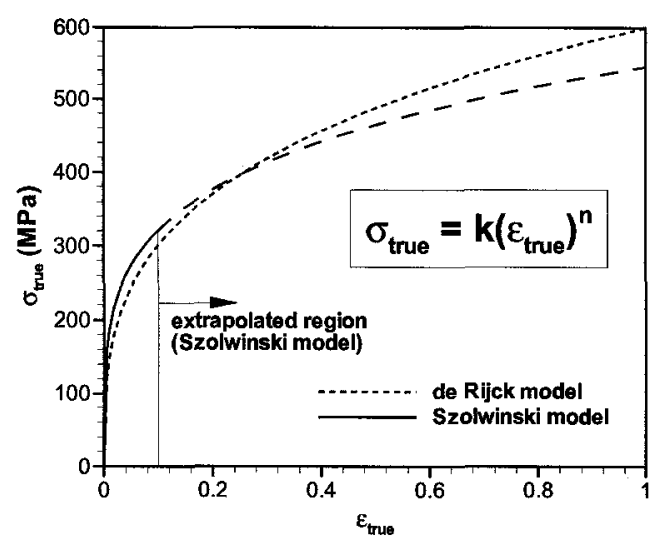

Figure 4.5: Comparison of 2117-T4 rivet material model true stress-strain curves

utilized compression tests on billet samples extracted from actual rivet shanks. The experimental data, however, was limited to a strain range of $0.02 \leq \epsilon_{\text {true }} \leq 0.10$ bringing into question the validity of extrapolating the model for higher strains which would be present during rivet forming. The second model, developed by de Rijck [2], utilized experimental driven rivet head geometries and simplified analytical models for the deformation of the rivet shank; however, the model became available only after the current study was nearly complete. Good agreement was found between the two models over the strain range of Szolwinski's experimental data; however, a lower strain hardening behaviour was observed in the extrapolated region of Szolwinski's model (Figure 4.5). Despite the differences in the material models, FE simulations repeated using de Rijck's model showed negligible difference in rivet deformation and resulting residual stress state in the 2024-T3 plates; thus only results from the original simulations utilizing Szolwinski's 2117-T4 rivet material model will be presented. A summary of the material models used is given in Table 4.1.

\subsubsection{Delamination Modelling for GLARE}

Delamination between the various plies within the GLARE laminate was not considered in the rivet installation simulations. From the experiences gained in simulating delamination during the cold expansion process, it was found that requirements for accurately predicting delamination size conflicted with those for accurately predicting residual stress. To satisfy 
Table 4.1: Summary of FE material models $[7,45,46]$

\begin{tabular}{ll}
\hline Material Model Parameter & Value \\
\hline 2024-T3 (Power Law Plasticity) [45] & \\
Elastic modulus, $E$ & $72.4 \mathrm{GPa}$ \\
Strength coefficient, $k$ & $530 \mathrm{MPa}$ \\
Hardening exponent, $n$ & 0.10 \\
Poisson ratio, $\nu$ & 0.33 \\
Unidirectional Prepreg (Orthotropic Elastic) $[46]$ & \\
Local 11 elastic modulus, $E_{11}$ & $50.6 \mathrm{GPa}$ \\
Local 22 elastic modulus, $E_{22}$ & $9.9 \mathrm{GPa}$ \\
Local 33 elastic modulus, $E_{33}$ & $9.9 \mathrm{GPa}$ \\
Local 12 shear modulus, $G_{12}$ & $3.7 \mathrm{GPa}$ \\
Local 23 shear modulus, $G_{23}$ & $3.7 \mathrm{GPa}{ }^{*}$ \\
Local 31 shear modulus, $G_{31}$ & $3.7 \mathrm{GPa}$ \\
Local 21 Poisson ratio, $\nu_{21}$ & 0.063 \\
Local 31 Poisson ratio, $\nu_{31}$ & 0.063 \\
Local 32 Poisson ratio, $\nu_{32}$ & 0.32 \\
2117-T4 (Power Law Plasticity) [7] & \\
Elastic modulus, $E$ & $71.7 \mathrm{GPa}$ \\
Strength coefficient, $k$ & $544 \mathrm{MPa}$ \\
Hardening exponent, $n$ & 0.23 \\
Poisson ratio, $\nu$ & 0.33 \\
\hline
\end{tabular}

${ }^{*} G_{23}$ was not provided in [46], so it was estimated to be equal to $G_{12}$ and $G_{31}$. Finite element results were found to be insensitive to variations of $\pm 10 \%$ in $G_{23}$.

both would result in unfeasible computational times. To overcome this problem in the cold expansion simulations, a simplified delamination model requiring knowledge of the final delamination size was used. For the case of riveting, however, there is no prior knowledge of the delamination size. If a delamination is formed, it is hidden by the rivet heads. Removing the rivet to assess the delamination is not an option as it would result in further elastic springback of the plate. In Chapter 3 , it was observed that unrestrained elastic springback of the fibre layer results in large shear stresses that could create or further propagate an existing delamination. For these reasons, delamination was excluded from the simulations. 


\subsubsection{Model Verification}

Sensitivity studies on mesh size, simulation time, and other simulation parameters was performed to ensure accuracy in the analysis results. For conciseness, only results from the refined finite element model are presented here. Details and results from the refinement process are described in Appendix A.

Experimental verification of the finite element model was not a trivial task. The residual stress results of interest are hidden beneath the manufactured and driven rivet heads, making most experimental stress analysis techniques unfeasible. Stresses and strains beyond the rivet head can easily be determined experimentally; however, this is not an adequate measure for verifying the performance of the model underneath the rivet head where stress and strain magnitudes and gradients can be greater by an order of magnitude or more. Furthermore, no analytical models exist for predicting riveting induced residual stresses. As a result, directly verifying the finite element results was not possible. Instead, confidence was gained through several indirect verification studies.

Firstly, the performance of the material models was verified through the cold expansion finite element simulations described in Chapter 3. The existence of analytical solutions for uniform cold expansion processes permitted direct verification of the finite element residual stress results. As the radial expansion mechanism for forming residual stress during riveting and cold expansion are analogous, verification of the cold expansion simulations provides an increased confidence in the performance of the rivet installation simulations.

Secondly, the geometry of the rivet installation finite element model was modified to allow the residual stress results to be compared to an existing experimental investigation. Most experimental stress analysis techniques provide inadequate results for verification purposes due to their inability to provide data in the region of interest beneath the rivet head. The method of neutron diffraction overcomes this limitation; however, the exorbitant costs of this technique made this method beyond the resources of this study. Fortunately, a neutron diffraction study on riveting induced residual strains completed by $\mathrm{Li}$, Shi, and 


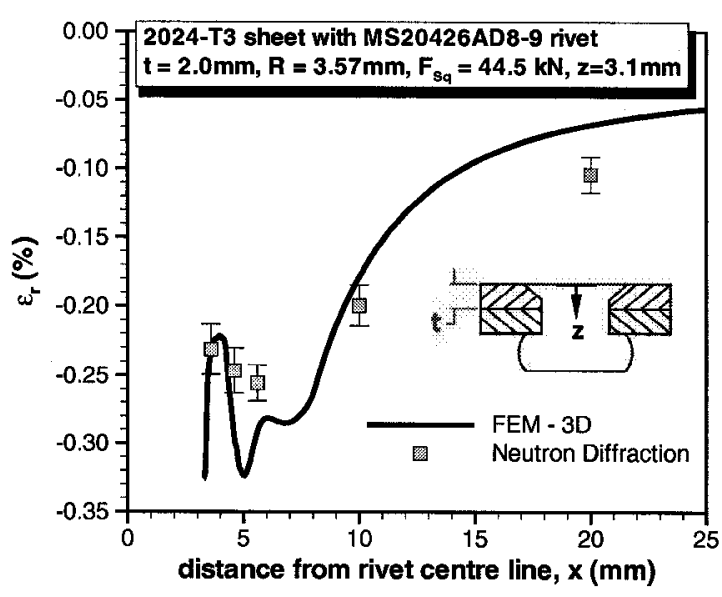

(a) radial strain

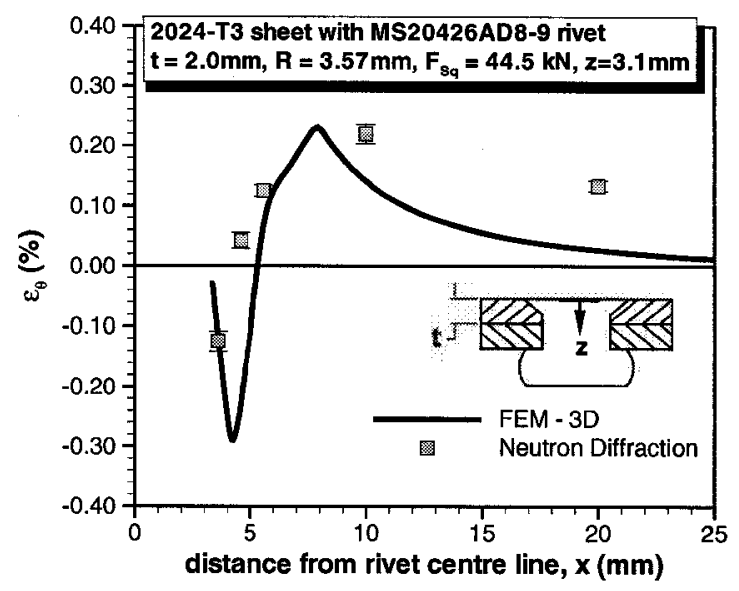

(b) tangential strain

Figure 4.6: Comparison of FE residual strains to neutron diffraction data $[16,50]$

Bellinger $[16,50]$ was available in the literature. Modifications to the current FE model geometry were implemented to represent the MS20426AD8-9 rivet and $2.0 \mathrm{~mm}$ thick 2024-T3 sheets used in their experimental study. Figure 4.6 shows a comparison of a subset of FE model results to the neutron diffraction data. A detailed comparison can be found in Appendix B. The residual strain magnitudes agree well with the FE model, with some discrepancies occurring near sharp peaks in strain $(x=4-5 \mathrm{~mm})$. Capturing these sharp peaks using the neutron diffraction method becomes difficult due to the required gauge volume over which the residual strain is determined for each data point. Thus, sharp peaks are lost in the neutron diffraction data due to volume averaging. This neutron diffraction study used a gauge volume of $1 \mathrm{~mm}^{3}$. Further details on the exact geometries of these gauge volumes can be found in references $[16,50]$.

Finally, the load-deflection behaviour of the bucking bar, a common benchmark used in previous finite element studies, was analyzed. The load-deflection behaviour of the bucking bar was obtained experimentally using a MTS servo-hydraulic test frame fitted with standard riveting tools and is shown in Figure 4.7 alongside the FE results. Good agreement is observed with the experimentally obtained curve with less than a $5 \%$ error, verifying the stiffness and plasticity of the rivet material model. 


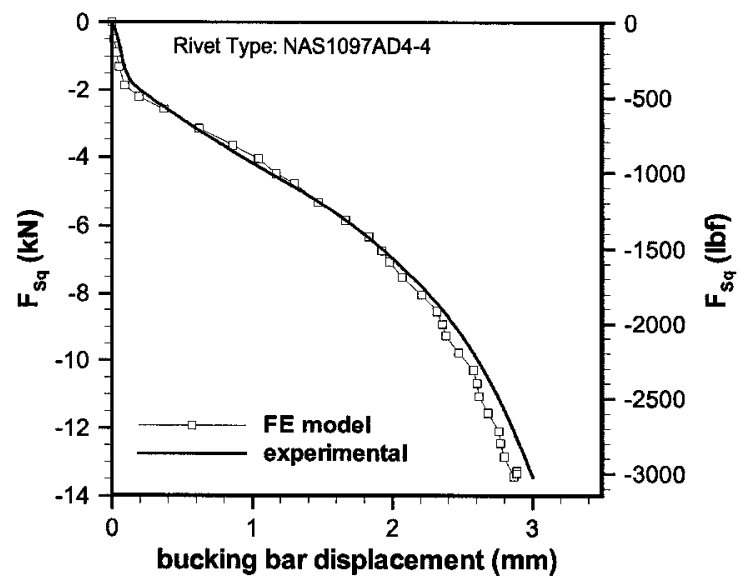

Figure 4.7: Comparison of FE and experimental bucking bar force-deflection curves

Although no direct verification of the results for the FE model in its configuration used for this study could be made, much effort has been placed in testing and validating indirectly all the aspects of the FE model. From the results of these efforts, confidence in the performance and results of the FE model has been established.

\subsection{Finite Element Results}

The overall results and conclusions reached during the present finite element study are discussed in the following section. Only a subset of the results will be presented as needed to support the findings presented. Additional results are presented in Appendix $\mathrm{C}$ for completeness.

For ease of discussion, results will be first discussed in context of the simulations containing 2024-T3 aluminum as the sheet material. Differences observed for simulations containing GLARE3-2/1-0.3 sheet will be discussed in Section 4.4.5.

\subsubsection{Hole Expansion}

The degree of hole expansion resulting over the range of rivet squeeze forces for the universal and countersunk rivet cases is shown in Figure 4.8. Two additional dash-dot-dot lines are 


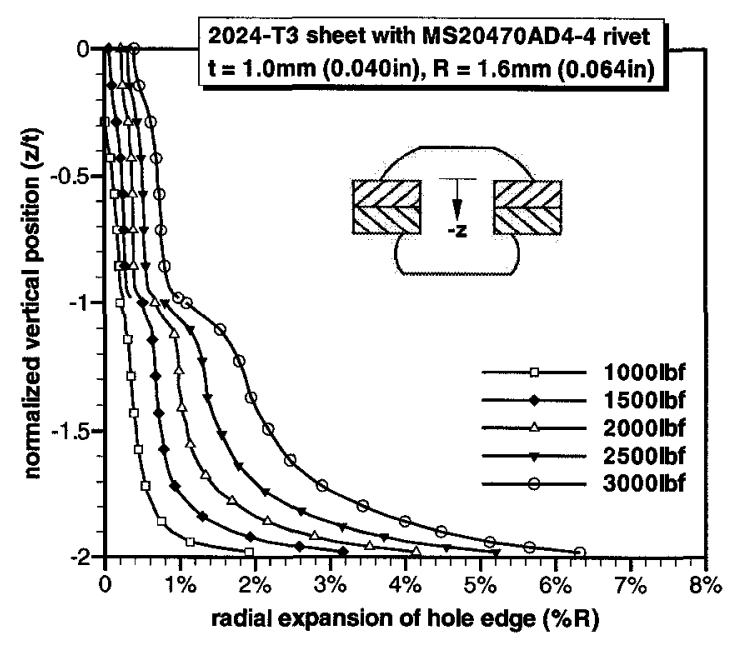

(a) universal rivet

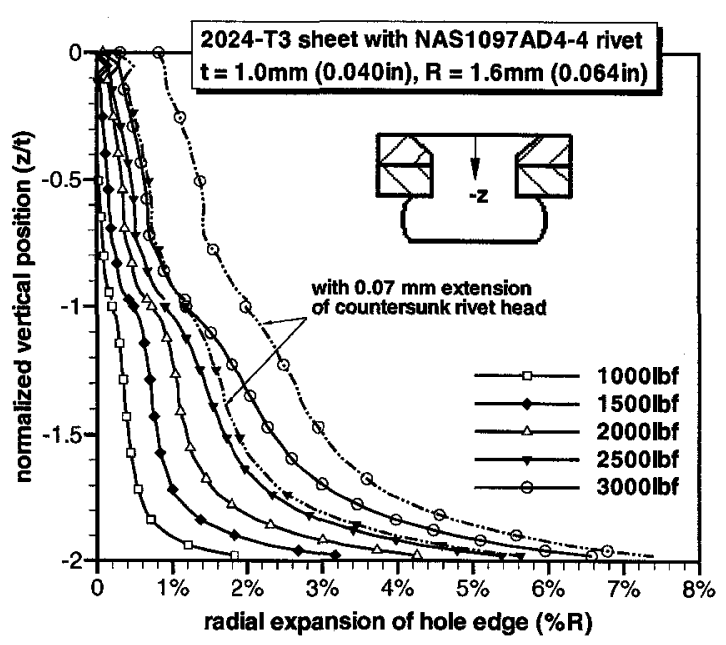

(b) countersunk rivet

Figure 4.8: Radial expansion of rivet holes for universal and countersunk rivets at various squeeze forces

plotted in Figure 4.8b illustrating the difference in the level of hole expansion at high squeeze forces between countersunk rivets that are perfectly flush before installation and rivets with a $0.07 \mathrm{~mm}$ extension above the joint surface before installation.

As expected, there is a large disparity in expansion level between the outer and inner sheet for all rivet cases. Improved hole filling and greater expansion levels are expected in the inner sheet due to its proximity to the deformable rivet head. As the manufactured rivet head is fully seated by the outer sheet for the countersunk rivet case, or rivet set for the universal rivet case, it is not as free to deform. Thus, the manufactured rivet head constrains radial expansion of the rivet shank, resulting in larger expansion levels towards the free end of the rivet which produces the driven rivet head.

Expansion levels in the outer sheet are generally low. Less than $1 \%$ radial expansion was observed for all cases with exception of the countersunk rivet with a $0.07 \mathrm{~mm}$ extension above the joint surface. This exception will be further discussed in Section 4.4.3. Cold expansion processes typically introduce a 3-5\% expansion level and allow full elastic springback of the surrounding sheet material. The lower expansion levels due to rivet installation and the presence of the rivet which restricts springback of the sheet indicate that smaller and less 
favourable regions of residual stress would be expected during riveting using only the radial expansion analogy.

Negligible differences in expansion levels were observed for universal and countersunk rivets, with the exception of the countersunk rivet with a $0.07 \mathrm{~mm}$ extension at high squeeze forces. These results contradict the belief that improved hole filling and interference can be achieved through the use of universal rivets. Expansion levels do not seem to be reduced along the conical recess of the outer sheet. As the expansion levels in the outer sheet were in general quite low, this finding may not hold for higher squeeze forces where the driving force for radial expansion in the outer sheet is expected to be larger. The current study, however, did examine a range of squeeze forces that bound the current acceptable range of driven rivet head dimensions. The countersink depth may also influence this result. The countersink depth used in this study ensures that a portion of the outer sheet rivet hole remains cylindrical. Radial expansion along this cylindrical portion of the hole could influence the overall expansion of the outer sheet. Again, it is common practice to leave a section of the outer sheet rivet hole cylindrical, so these results are representative of current riveting practice.

\subsubsection{Generalized Residual Stress Distribution}

A typical residual tangential and radial stress distribution observed in the FE models is shown in Figure 4.9 and has been divided into four zones for discussion purposes. Zones 1-3 correspond to the regions surrounding the rivet which undergo plastic deformation during riveting while Zone 4 is the region of elastic deformation. Within the plastic region, Zone 1 and 2 experience large through-thickness compressive stresses as a result of a portion of the rivet squeeze force being transmitted through the driven rivet head into the underlying sheets. The magnitude of this through-thickness stress was found to exceed the radial and tangential components by as much as a factor of two under the applied squeeze force, indicating its dominant role in the plasticity of these zones. This is illustrated in Figure 4.10 , which shows the stress distribution in the sheet while the rivet is under maximum applied squeeze force. Beyond the driven rivet head, plastic deformation still occurs in 


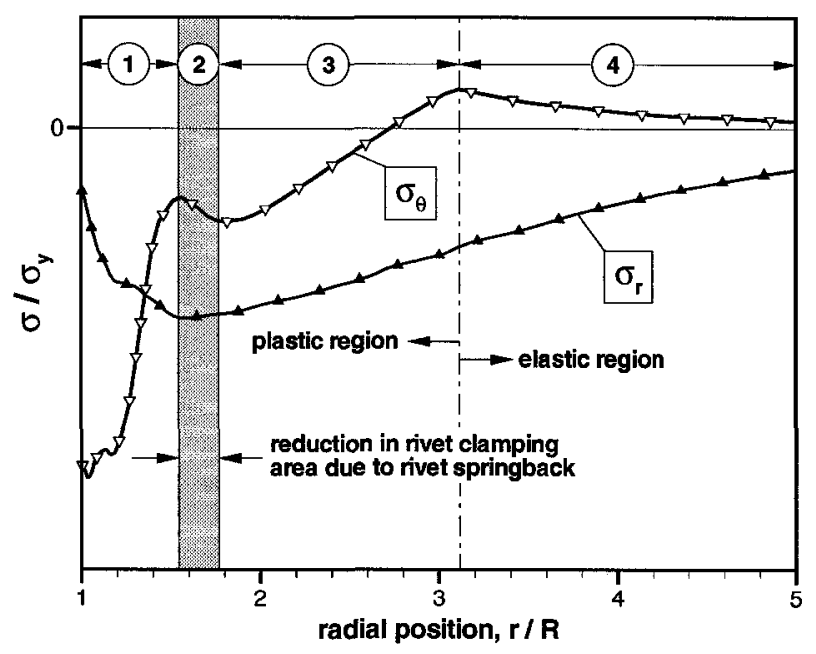

Figure 4.9: Schematic of typical residual tangential and radial stress distributions

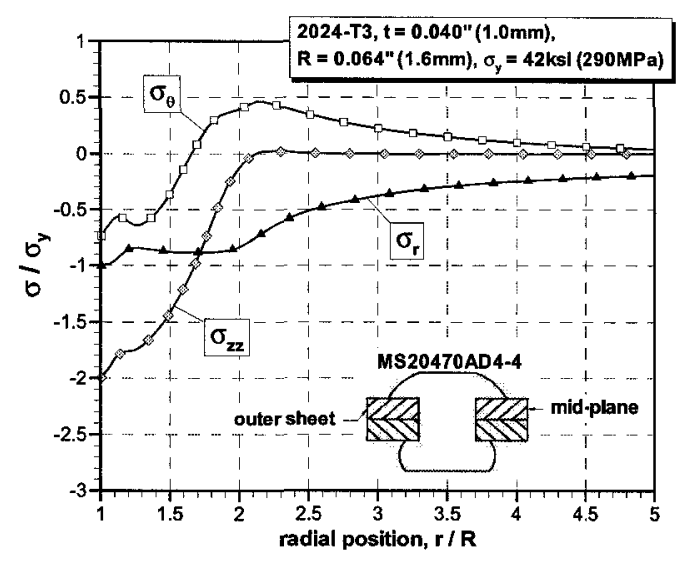

Figure 4.10: Outer sheet mid-plane stresses under maximum applied squeeze force

Zone 3. The through-thickness stress component drops off in this region and plasticity occurs due to radial expansion from the rivet and plastic flow of sheet material from Zones 1 and 2.

The importance of the through-thickness stress component on the formation of residual stresses is often overlooked in discussions on riveting. Within this study, however, it was found to contribute significantly to the plastic deformation of the sheet beneath the rivet head. The effect of through-thickness compression on the residual tangential stress distribution is clearly seen in Figure 4.9. A steeper residual tangential stress gradient was 
observed in the regions of high through-thickness stress, resulting in larger residual compressive stresses at the hole edge. Effects on the residual radial stress are not as clear. Generally, the magnitude of the residual radial stresses is larger and does not drop to zero at the hole edge as a result of the constraint provided by the installed rivet.

The effects of the through-thickness stress is observed away from the rivet heads as well. Plastic flow of the sheet material, constrained by the rivet heads in Zones 1 and 2 , provides an additional radial expansion component that increases the overall radius of the plastic region. Figure 4.11 illustrates this effect by comparing the observed mid-plane plastic zone radii for a universal rivet to predictions made with Ball's [10] analytical cold expansion model. For the rivet installation case, the plastic radii are plotted against the average radial expansion level found in the inner sheet.

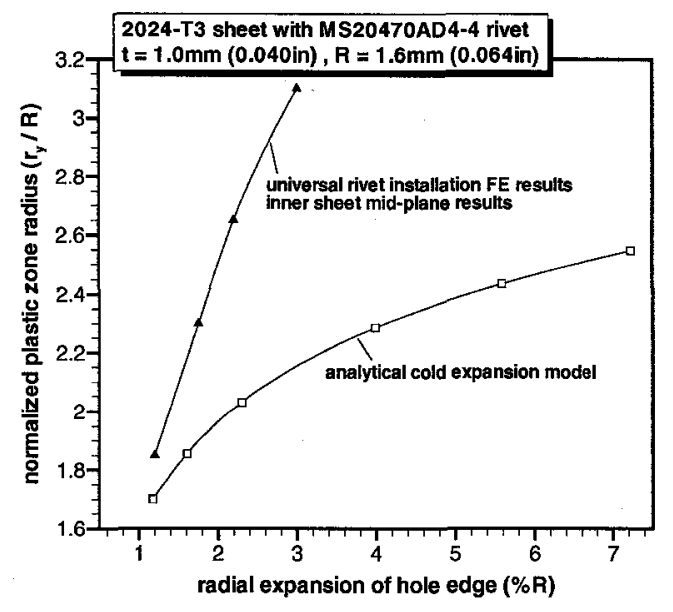

Figure 4.11: Comparison of cold expansion analytical model and riveting FE model results of plastic zone radius vs. hole expansion

\subsubsection{Effect of Countersink Depth}

Standard riveting practice places constraints on countersink depths that ensure a small extension of the rivet head above the joint surface prior to riveting. This practice ensures that the countersunk depth of the sheet does not exceed the height of the countersunk rivet head, which would result in poor hole filling within the countersunk sheet during riveting. Additionally, too deep a countersink would result in the rivet being lifted out 
of the countersink during riveting, reducing the benefit of through-thickness compressive stresses described above. In order to study the effect of this extension, finite element simulations were carried out for perfectly flush countersunk rivets and countersunk rivets with a $0.07 \mathrm{~mm}$ extension above the joint surface. Comparison of these two cases will be discussed here.

Comparison of the radial expansion levels for the two countersink depth cases shown in Figure $4.8 \mathrm{~b}$ shows that results for the two cases diverge at squeeze forces above $2000 \mathrm{lbf}$. At these higher squeeze forces, frictional slip occurs during rivet compression (Phase 3 in Figure 2.4) causing the countersunk sheet to slide up the countersunk rivet head and expand through a wedging action (wedge expansion). This wedge expansion mechanism is illustrated in Figure 4.12. This squeeze force also corresponds to the point at which the driven rivet head diameter exceeds that of the countersunk rivet head, which may be a contributing factor to the onset of wedge expansion.

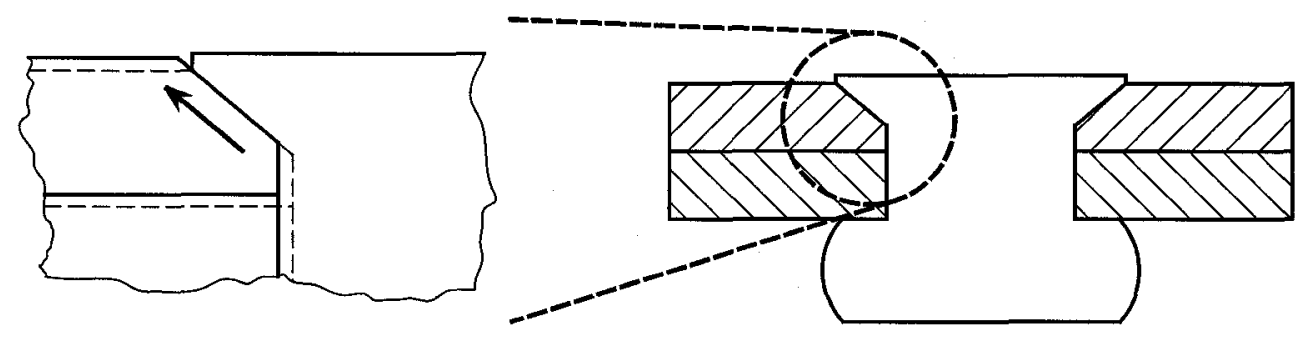

Figure 4.12: Illustration of wedge expansion mechanism

Similar to the radial expansion results, the residual stress results also differ when the wedge expansion mechanism is present. This is illustrated in Figure 4.13 where faying surface residual stresses in the outer sheet are plotted for the two countersink depth cases installed with a squeeze force of $3000 \mathrm{lbf}$. For the perfectly flush case, expansion levels are low in the outer sheet, resulting in the lack of a radial expansion dominated zone (Zone 3) as described in the previous section. A significant residual radial stress is also present at the hole edge as a result of the rivet shank restricting the elastic springback of the surrounding sheet. For the $0.07 \mathrm{~mm}$ extension case, the additional expansion provided by the wedge expansion mechanism results in the addition of a well defined radial expansion dominated 


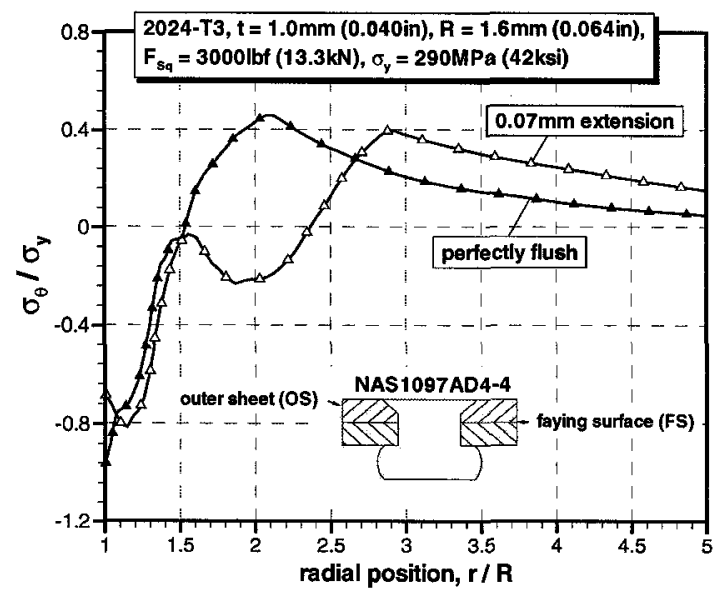

(a) tangential stress

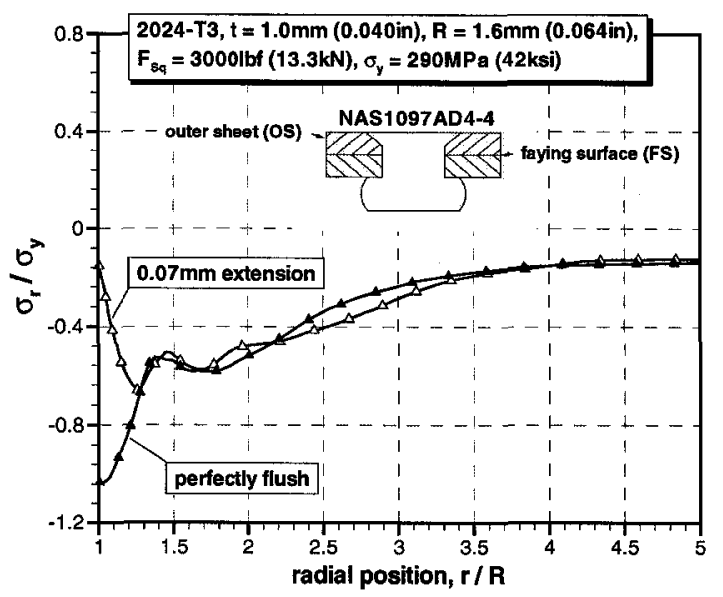

(b) radial stress

Figure 4.13: Influence of countersink depth on outer sheet faying surface residual stress

zone shown in Figure 4.13a. This added zone increases the region of plastic deformation and produces a larger area of beneficial residual compressive stresses. The residual radial stress at the hole edge also drops as a result of a disparity in the expansion levels between the rivet shank and surrounding sheet. The wedge expansion mechanism increases the expansion level in the sheet, but does not affect the expansion level in the rivet shank. This disparity allows an increased amount of elastic springback in the sheet to occur before it is restrained by the rivet shank.

In addition to the wedge expansion mechanism, a small extension of the rivet head localizes the through-thickness compressive stress resulting from the squeeze force. For the perfectly flush case, the rivet set contacts both the rivet and the surrounding outer sheet, distributing the rivet squeeze force over the entire area of the rivet set. Extension of the rivet head above the joint surface eliminates contact between the rivet set and the outer sheet, localizing the transfer of force into the sheets to the conical contact region between the rivet and outer sheet. This would contribute to the formation of residual stresses beneath the rivet head; however, it is believed that the wedge expansion mechanism accounts for the majority of the variation observed between the two countersink depth cases.

Existing fatigue data supports the influence of countersink depth. Experimental studies carried out by Müller [1] found a correlation between the amount of rivet head protrusion, 
rivet squeeze force, and a shift in fatigue cracking from the countersunk to non-countersunk sheet in riveted splices containing NAS1097 style countersunk rivets. He attributed this effect to changes in secondary bending resulting from a permanent curvature (or imperfection as it was called) formed by bending of the inner and outer sheets around the protruding countersunk rivet head at high squeeze forces. The increase in compressive hoop stresses within the countersunk sheet resulting from the wedge-expansion mechanism could also contribute to this shift in fatigue cracking. Additionally, the onset of formation of an imperfection was also observed by Müller to occur when the driven rivet head exceeded the countersunk rivet head, which also agrees with the onset of the wedge-expansion mechanism in the present study.

$\mathrm{Li}$ and Shi $[15]$ also investigated the residual stress state resulting from the installation of a countersunk rivet that protruded $0.07 \mathrm{~mm}$ above the sheet surface; however, there was no evidence of the wedge-expansion described above. Several key differences in their model could have contributed to this finding. First, Li and Shi considered a MS20426 style countersunk rivet, which has a larger countersunk head than the NAS1097 style considered in the current investigation. As a result, the driven rivet head diameter did not exceed the countersunk rivet head diameter in their simulations. Bending in the outer sheet associated with this event could be critical in initiating frictional slip between the outer sheet and countersunk rivet head. Second, the depth of the countersink was equal to the thickness of the outer sheet in Li and Shi's model. The absence of a cylindrical hole surface in the outer sheet removes the contribution of radial pressure along this surface to frictional slip.

\subsubsection{Faying Surface Residual Stresses}

Knowledge of the residual stress distribution along the faying joint surfaces is important as they are likely locations for crack nucleation. Peak tensile secondary bending stresses in addition to fretting damage occur at the faying surfaces, contributing to this likelihood. The introduction of compressive residual stresses in this region, particularly tangential to the rivet hole, can greatly affect crack nucleation and early growth of any fatigue crack that forms. 


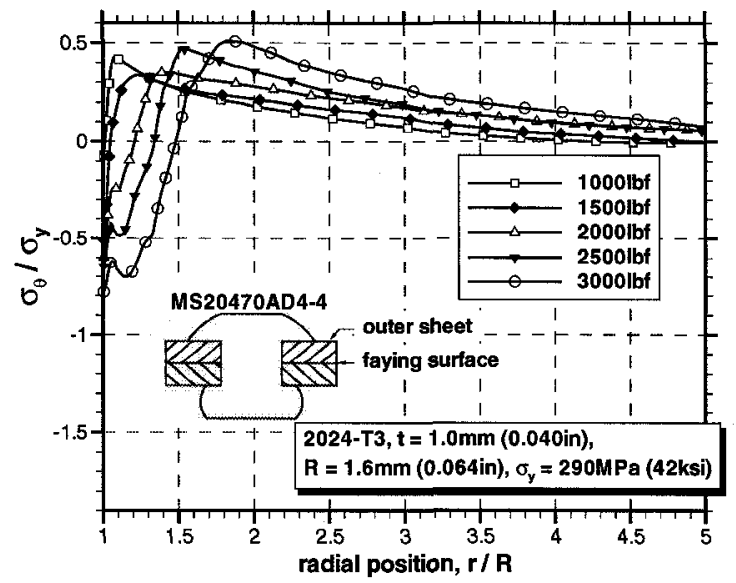

(a) universal rivet, outer sheet

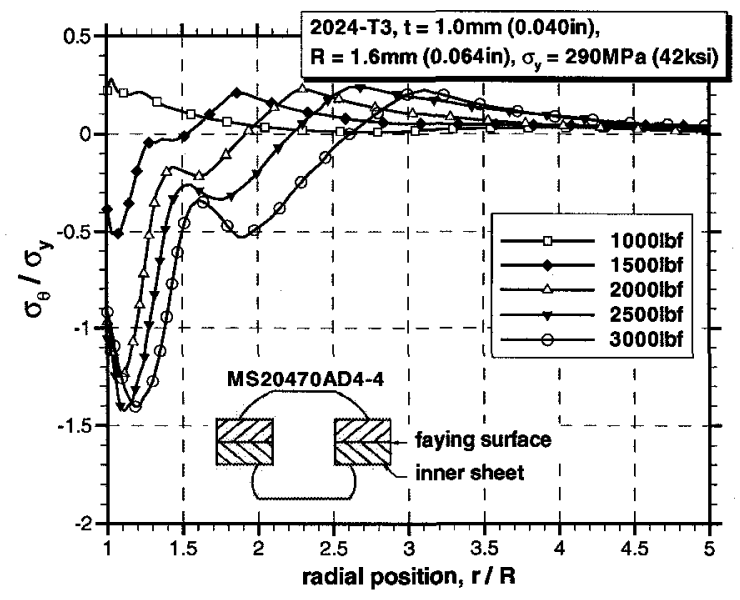

(c) universal rivet, inner sheet

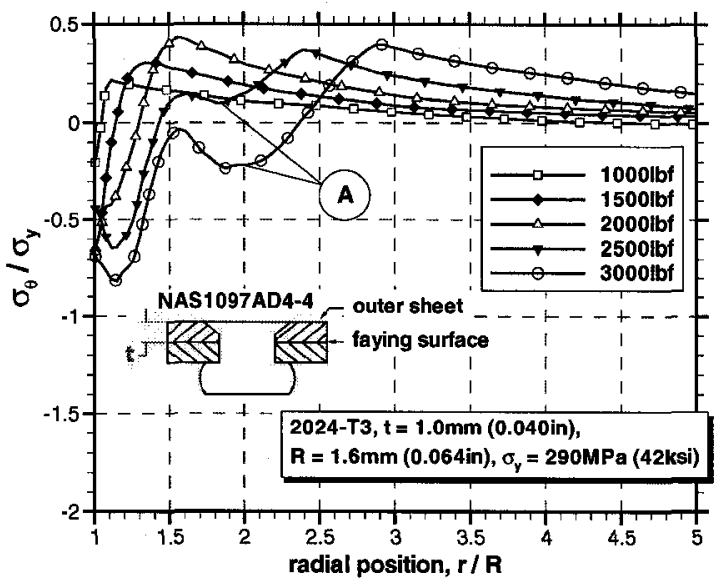

(b) countersunk rivet, outer sheet

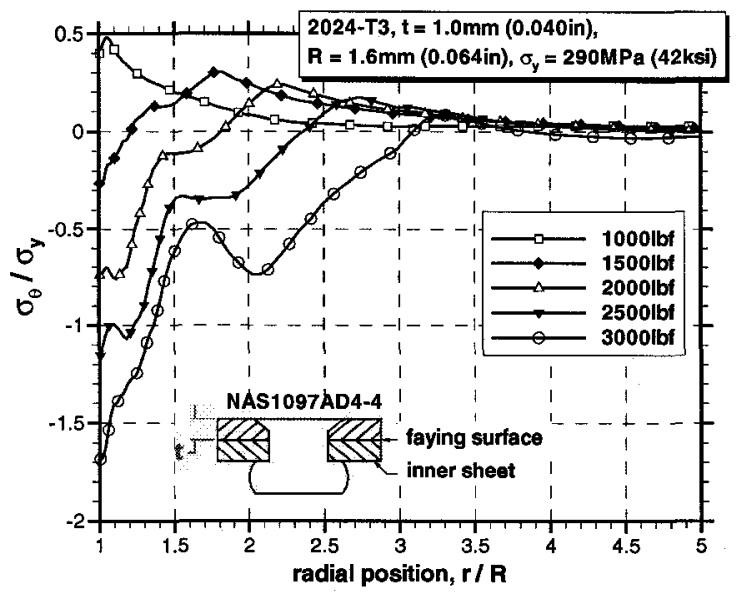

(d) countersunk rivet, inner sheet

Figure 4.14: Faying surface residual tangential stress distribution in 2024-T3 sheet for various rivet squeeze forces 
The faying surface residual tangential stress distributions observed for the universal and countersunk rivet cases are shown in Figure 4.14. Results for the perfectly flush rivet case are not shown as this case cannot be achieved in practice.

The effects of some of the points discussed in the previous sections can be seen in the faying surface residual stress results. The outer sheet stress distributions (Figure 4.14 a and b) show a compressive residual stress field confined to the area between the rivet heads. This corresponds to the region of plasticity dominated by through-thickness compression of the sheets. Only in instances where the wedge expansion mechanism is present does the radial stress dominated plastic zone develop as highlighted by balloon $\mathrm{A}$ in Figure 4.14b. Conversely, the radial stress dominated plastic zone is present in the inner sheet for most examined cases. The exception being at the lowest squeeze force examined, $1000 \mathrm{lbf}$, where very little plastic deformation of the sheet near the faying surface occurs. This difference between the outer and inner sheets is due to the improved hole filling and higher radial expansion levels observed in the inner sheet. A consequence is the larger region and magnitude of residual compressive stress in the inner sheet, which contributes to the higher incidence of fatigue cracking in the outer sheet compared to the inner sheet.

Increasing the rivet squeeze force was found to increase the region and magnitude of compressive residual tangential stresses. Although no limit to this increase was identified, experience has shown that larger driven rivet head dimensions associated with larger squeeze forces tend to shift the crack nucleation location away from the fastener hole, negating the benefit of the residual compressive stresses. Under the footprint of the larger driven rivet head dimensions, peak secondary bending stresses resulting from rivet rotation flow around the fastener hole, driving the shift in crack nucleation location. This influence of rivet geometry and rivet squeeze force on secondary bending is investigated in Chapter 5 .

\subsubsection{Influence of Sheet Material}

Overall, GLARE3-2/1-0.3 exhibited similar trends as described above for 2024-T3 with respect to rivet type, countersink depth, and squeeze force. No significant differences in radial expansion levels were observed between the GLARE and 2024-T3 simulations. Effects 
of the prepreg layers, however, are evident when directly comparing results from the two sheet materials.

The faying surface residual tangential stress distributions observed for the universal and countersunk rivet cases in GLARE are shown in Figure 4.15. These can be directly compared to the results for 2024-T3 shown in Figure 4.14.

Comparison of these results show that riveting in GLARE results in larger regions of plasticity and smaller gradients in residual tangential stress, particularly towards the edge

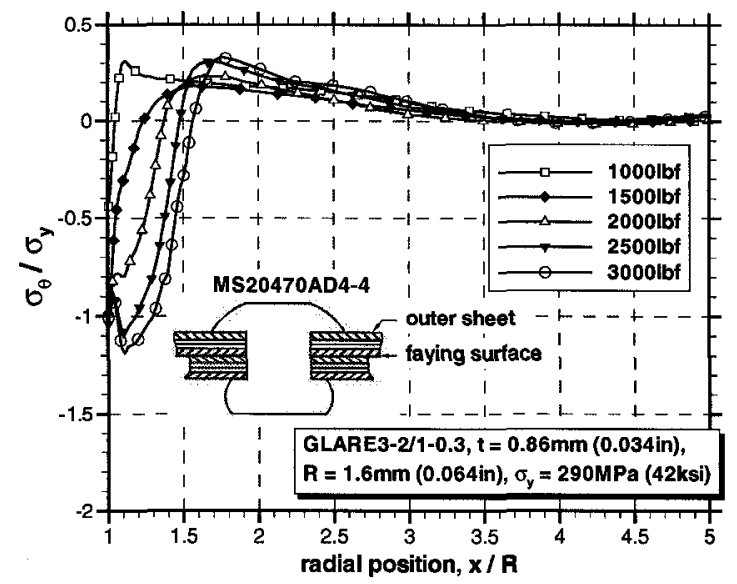

(a) universal rivet, outer sheet

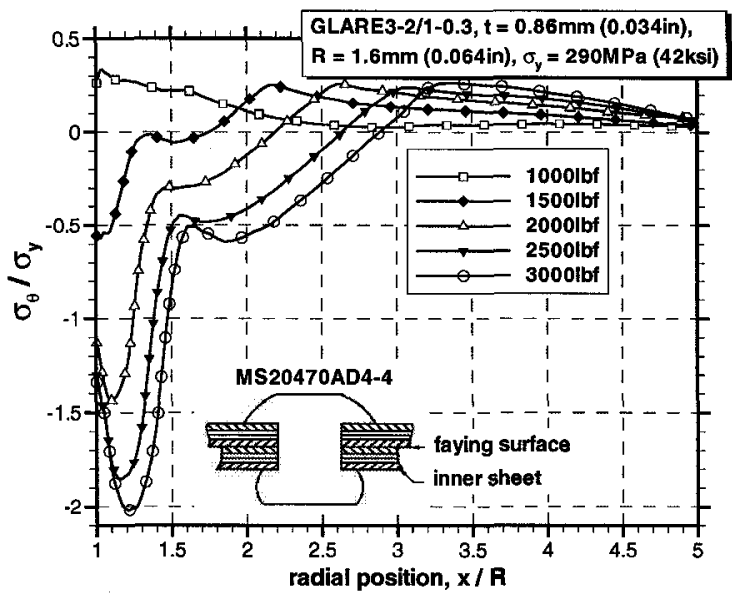

(c) universal rivet, inner sheet

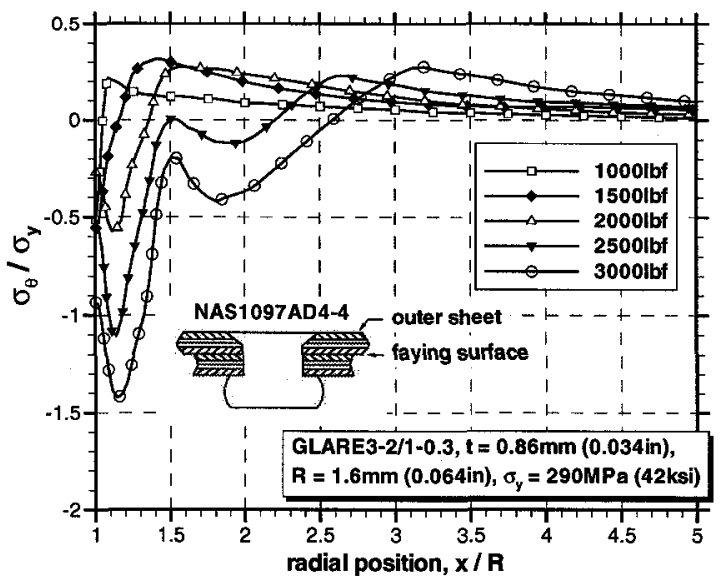

(b) countersunk rivet, outer sheet

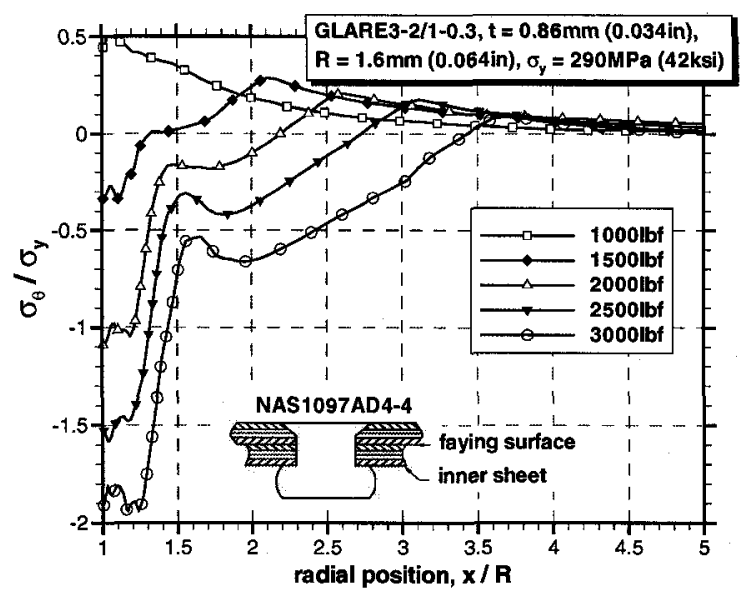

(d) countersunk rivet, inner sheet

Figure 4.15: Faying surface residual tangential stress distribution in GLARE3-2/1-0.3 sheet for various rivet squeeze forces 
of the plastic region. This behaviour can be attributed to the apparent strain hardening behaviour caused by the fibre layers. This influence of the fibre layer was previously discussed in Chapter 3.

Larger magnitudes of residual compressive tangential stresses are also evident near the hole edge for GLARE in Figure 4.15. Despite the lower stiffness of the prepreg layers, lack of plasticity allows them to store more elastic energy during riveting compared to the aluminum layers. As a result, the larger springback response of the prepreg layers is resisted by the aluminum layers resulting in larger compressive hoop stresses at the hole edge. Similarly, the elasticity of the prepreg layers produces a larger clamping force between the outer and inner sheet (Figure 4.16) as the rivet resists the through-thickness springback of the sheets. Although not a subject of the present study, this larger clamping force could have significant consequences related to frictional load transfer and fretting fatigue in GLARE riveted lap joints.

Anisotropy of the prepreg layers had a minimal influence on residual stresses in the aluminum layers near the rivet hole. This is illustrated in Figure 4.17 for the case of a $3000 \mathrm{lbf}$ installed universal rivet in GLARE. Further from the rivet hole, residual tangential stresses in the aluminum layers along the $\theta=45^{\circ}$ direction become slightly more tensile

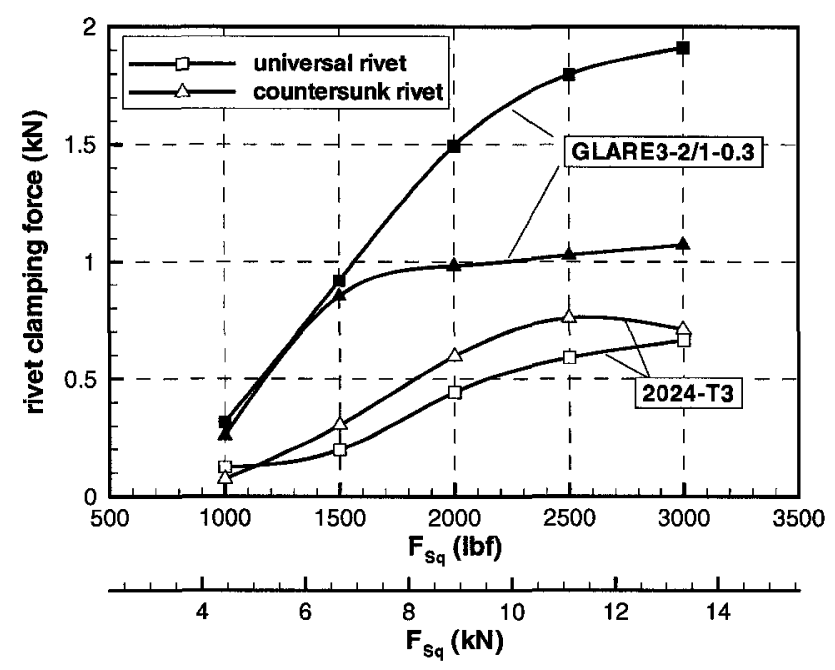

Figure 4.16: Comparison of residual rivet clamping force in 2024-T3 and GLARE3-2/1-0.3 sheet 


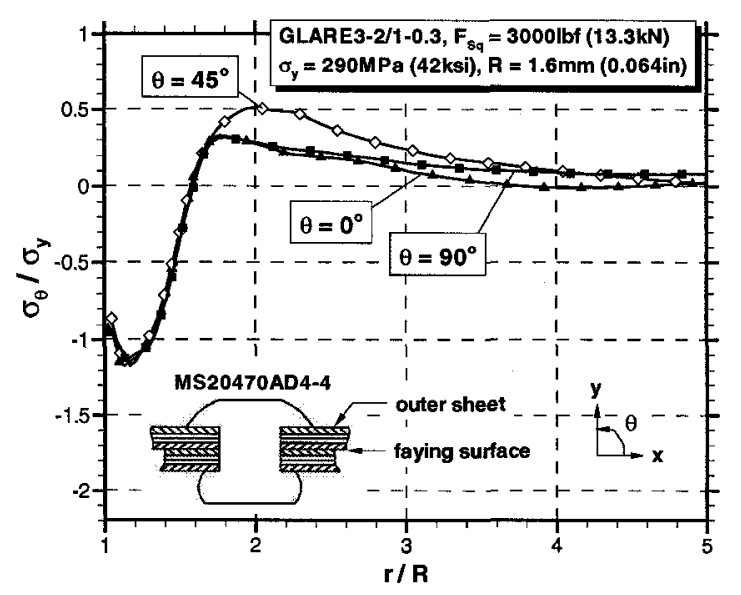

(a) universal rivet, outer sheet

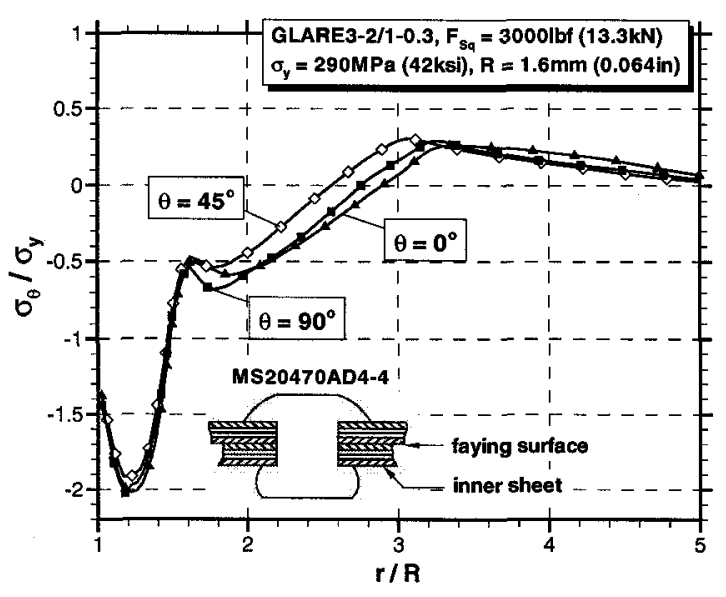

(b) universal rivet,inner sheet

Figure 4.17: Angular variation of residual tangential stress distribution in GLARE3-2/10.3 with a universal rivet

due to the reduced combined stiffness (and springback response) of the two prepreg layers in this direction. Similar trends were also observed for the countersunk rivet case.

\subsection{Conclusions}

A 3D finite element analysis has been carried out to investigate the influence of rivet installation on residual stresses in the context of universal and countersunk rivets installed in 2024-T3 and GLARE3-2/1-0.3. Based on the results of the finite element simulations, the following conclusions can be made:

- Large through-thickness stresses are generated in the joint sheets underneath the rivet head during rivet installation, contributing to plasticity within this region. Gradients and magnitudes of the resulting residual hoop stress field in this region are larger than those predicted by radial expansion models, highlighting the importance of these through-thickness stresses in riveted joints.

- Increases in the rivet squeeze force result in larger regions and magnitudes of residual compressive hoop stresses. Within the current study, no limiting or optimum squeeze force has been identified. 
- The importance of the countersink depth relative to the countersunk rivet head height prior to installation was confirmed by the present investigation. Additionally, limited protrusion of the countersunk rivet head above the joint surface was found to result in expansion of the outer sheet through a wedging action at high rivet squeeze forces. This expansion mechanism may be related to the geometric ratio of the driven and manufactured rivet head; however, further investigation is required.

- Universal rivets do not provide any significant increase in expansion of the outer joint sheet compared to countersunk rivets. In instances where the wedging expansion mechanism was present, countersunk rivet provide improved expansion and residual compressive tangential stresses in the outer joint sheet. Although the fatigue performance of universal joints is still superior to countersunk joints due to a lack of a stress concentration due to countersinking, this result indicates that countersunk joints could exhibit a larger benefit due to riveting induced residual stresses.

- The apparent strain hardening behaviour of the prepreg layers results in larger regions of plastic flow (and compressive residual tangential stresses) in GLARE compared to 2024-T3.

- Elastic springback of the prepreg layers is resisted by the less resilient aluminum layers/rivet, resulting in larger residual compressive tangential stresses and larger rivet clamping forces than riveting in 2024-T3.

- Anisotropy of the prepreg layers in GLARE has a minimal effect on the residual tangential stress distribution, particularly close to the rivet hole.

The results from this chapter provide insight into the role of rivet installation on the formation of residual stresses in a riveted lap joint. Residual stress formation, however, does not fully encompass the influence of rivet installation on the fatigue behaviour of riveted joints. Building on these residual stress results, the next chapter further examines the role of rivet installation on fatigue by investigating its effects on secondary bending in a loaded lap joint. 


\section{Chapter 5}

\section{Secondary Bending and Splice Loading}

\subsection{Introduction}

Load path eccentricities inherent in lap joint designs produce bending stresses which contribute to the nucleation and growth of fatigue cracks. This bending mechanism, known as secondary bending, was introduced earlier in Section 2.4.2. Current understanding of the influence of rivet type and installation on secondary bending is limited and is primarily based on qualitative assessments of fatigue failures.

This chapter presents a finite element study undertaken to investigate the role of rivet type and installation on secondary bending in a loaded riveted lap joint (or splice). First, a brief overview of the current state of the art in secondary bending research will be presented. The finite element model and its results are then presented and the role of rivet type and installation on these results is discussed. As in previous chapters, the finite element study was completed for both monolithic 2024-T3 and GLARE3-2/1-0.3 sheet materials in order to investigate potential differences in secondary bending for the two sheet materials.

\subsection{Review of Secondary Bending}

The primary means currently used for predicting the effects of secondary bending is the neutral line model $[1,2,8]$. The formulation of this model was presented earlier in Section 2.4.2. The main limitation of the neutral line model is its 1-dimensional formulation, which 
prevents the inclusion of the effects of discrete fastener locations. The neutral line model in effect represents a riveted lap joint with discrete fastener locations of finite size as a lap joint with infinitely thin and rigid bond lines along the fastener row locations. Despite this limitation, the neutral line model has been a useful tool for lap joint design. It provides a simple way of accessing the effect of rivet row spacing, sheet and doubler thickness, and other joint design variables on secondary bending. In the context of the present study, however, the simplifications within the neutral line model make it incapable of providing results which help assess the role of rivet type and installation on secondary bending.

Few investigations have considered the influence of a discrete fastener location on secondary bending in a riveted lap joint. Müller [1] provides a qualitative discussion on how the constraint provided by a rivet head can cause the maximum secondary bending stresses to flow around the rivet hole rather than intersect it (see Figure 2.10 in Section 2.4.2). Experimental observations of crack nucleation locations formed the basis for his discussion. Fawaz [51] investigated the variation in bending stress around the perimeter of a rivet hole using 3-dimensional finite element methods. Within the model, rivet installation was not explicitly simulated and slug-type rivets were defined with an as-installed geometry. The formation of residual stress was simulated by applying a thermal load to the fastener which resulted in a uniform radial expansion of the surrounding sheet. The effect of two rivet geometries, representative of a high and low rivet squeeze force, on secondary bending stresses at the hole perimeter were investigated. As the study primarily focused on bending stresses at the hole perimeter, limited information was gained regarding the qualitatively defined shift in maximum bending discussed by Müller.

Given the limited quantitative results related to the influence of rivet type and installation on secondary bending, the development of a 3-dimensional finite element model to study such effects was deemed to be a valuable contribution. The development of this model and the results obtained from it will be discussed in the following sections. 


\subsection{Splice Loading Finite Element Model}

To investigate the role of rivet installation on secondary bending, the finite element model presented in Chapter 4 was extended to simulate the assembly and subsequent loading of a riveted lap joint. The remainder of this section provides an overview of the development and verification of this finite element model.

\subsubsection{Model Description}

A two-row lap joint configuration was chosen for the finite element model, consisting of two sheets joined by $3.2 \mathrm{~mm}$ diameter 2117-T4 aluminum rivets. Although many structural joints in airframe applications adopt a 3- or more row configuration, the 2-row configuration is ideal for studying the effects of rivet installation on secondary bending because the load is divided equally between the two fastener rows. In configurations containing more than 2 rows of fasteners, the distribution of load transferred by each row is dependant on fastener flexibility as discussed in Section 2.4.1. Rivet installation is believed to influence fastener flexibility [1], however, this influence is beyond the scope of the present investigation. Use of the 2-row configuration thus allows the effect of fastener flexibility to be isolated from the investigation.

A schematic of the finite element model is shown in Figure 5.1, including an illustration of the applied boundary conditions and mesh density. Joints containing universal head (U.S. military specification MS20426AD4-4) and reduced-depth countersunk head (NAS1097AD4-4) rivets were modelled. For the countersunk rivet cases, the countersink depth in the sheet was specified to ensure a $0.07 \mathrm{~mm}$ protrusion of the countersunk head above the joint surface prior to installation. In addition to the two rivet types, 2024-T3 sheet with a nominal thickness of $1.0 \mathrm{~mm}$ and GLARE3-2/1-0.3 sheet with a nominal thickness of $0.86 \mathrm{~mm}$ were considered. A constant rivet pitch and row pitch of $25.4 \mathrm{~mm}$ and $38.1 \mathrm{~mm}$ was used for both cases. The presence of symmetry planes along the rivet centres and mid-rivet pitch lines was exploited, allowing the model to be reduced to a half-rivet pitch in width as illustrated in Figure 5.1. 


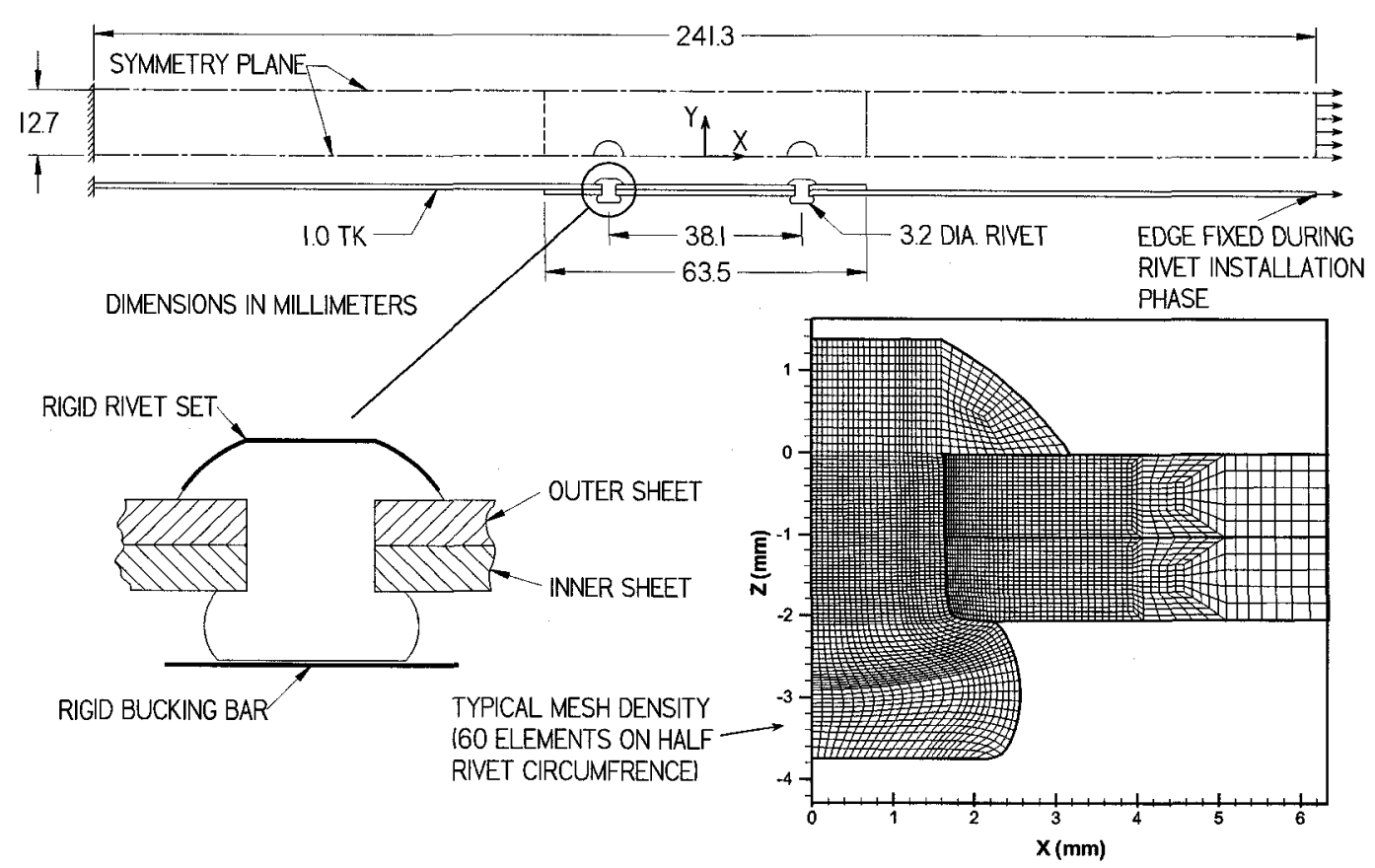

Figure 5.1: Schematic of splice loading finite element model

Element types, material models and contact/interface definitions for the 2-row splice model are identical to those presented for the rivet installation finite element model detailed in Section 4.3 and will not be repeated here.

In order to capture the effects of rivet installation, the finite element analysis was divided into two phases. First, rivet installation was simulated allowing the residual stress state prior to joint loading to be determined. Details regarding the rivet installation phase are identical to those presented for the rivet installation finite element model in Section 4.3 and will not be repeated here. As with the previous study, five squeeze forces were considered: $1000,1500,2000,2500$, and $3000 \mathrm{lbf}(4.4,6.8,8.9,11.1$, and $13.3 \mathrm{kN}$ respectively).

The second phase of the finite element analysis simulated the application of a uniaxial tension load on the lap joint model. Continuing from the final time step of the rivet installation phase, the rigid rivet set and bucking bar parts were deleted, and a tensile load was applied to the free end of the lap joint indicated in Figure 5.1. In all cases, a maximum tensile load of $100 \mathrm{MPa}$ was simulated. The tensile load was applied using a ramp function, including a dwell period at the maximum load to allow the system to come to rest. Similar 
to the rivet installation phase, quasi-static assumptions were also applied and simulation time was scaled to minimize processing time.

\subsubsection{Model Verification}

Sensitivity studies on mesh size, simulation time, and other simulation parameters was performed to ensure accuracy in the analysis results. For conciseness, only results from the refined finite element model are presented here. Details and results from the refinement process are described in Appendix A.

Validations of the finite element results were completed using several experimental methods. Verification studies carried out for the rivet installation phase of the finite element analysis were presented in Section 4.3.5 and only results from verification studies for the uniaxial tension loading phase will be presented here.

To verify the stress results obtained from the splice loading phase of the finite element analysis, surface strains in a loaded lap joint were determined experimentally using a Digital Image Correlation (DIC) technique $[52,53]$ at the National Research Council of Canada Institute for Aerospace Research. This technique uses a stereoscopic pair of digital cameras that track the motion of individual specks introduced onto a specimen using a high-contrast speckled paint. The stereoscopic camera pair tracks the motion of the specks in three dimensions, allowing in-plane strains and out of plane displacements to be determined. The DIC test setup and test coupon details are shown in Figure 5.2.

A comparison of the DIC and FE results are shown in Figure 5.3. DIC results are shown for all three rivet locations along the top rivet row. One of the limitations of the DIC method is that there is a noise floor below which strain readings cannot be made. For this particular setup, the noise floor was approximately 500 microstrains. Strain magnitudes and locations of peak strains agree well in the direction of load application, despite the presence of noise in the DIC results. In the transverse direction, the majority of the predicted strain values fall below the 500 microstrain noise floor of the DIC method, making it impossible to compare the strain magnitudes. Despite this limitation, the locations of peak strains are still discernible in the DIC results and agree well with the finite element predictions. 


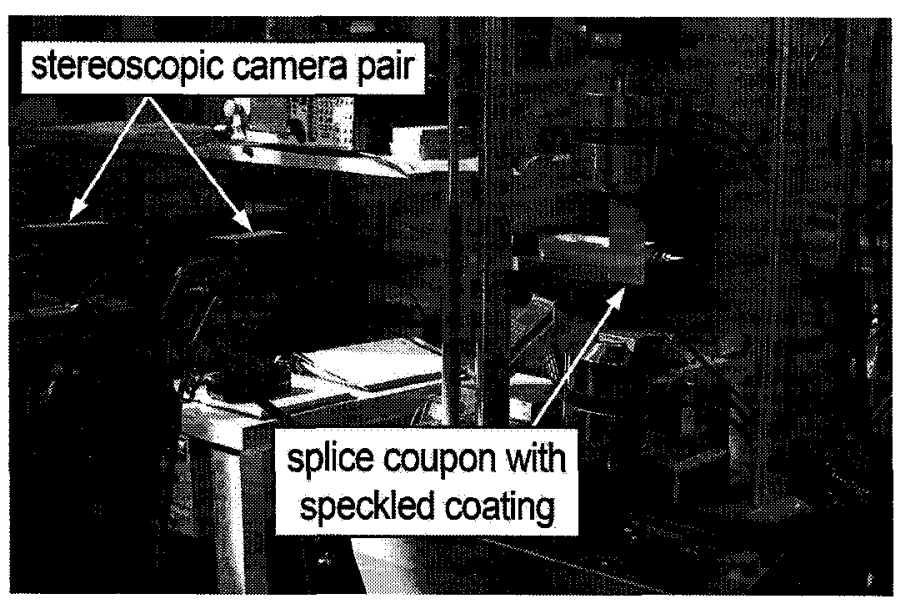

(a) DIC setup

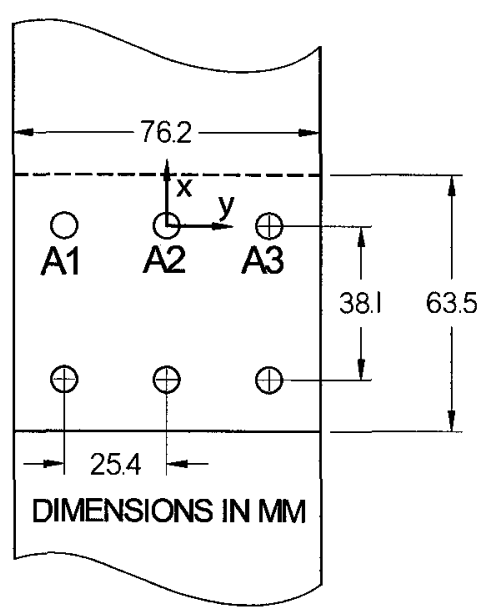

(b) DIC coupon

Figure 5.2: Test setup for DIC verification test

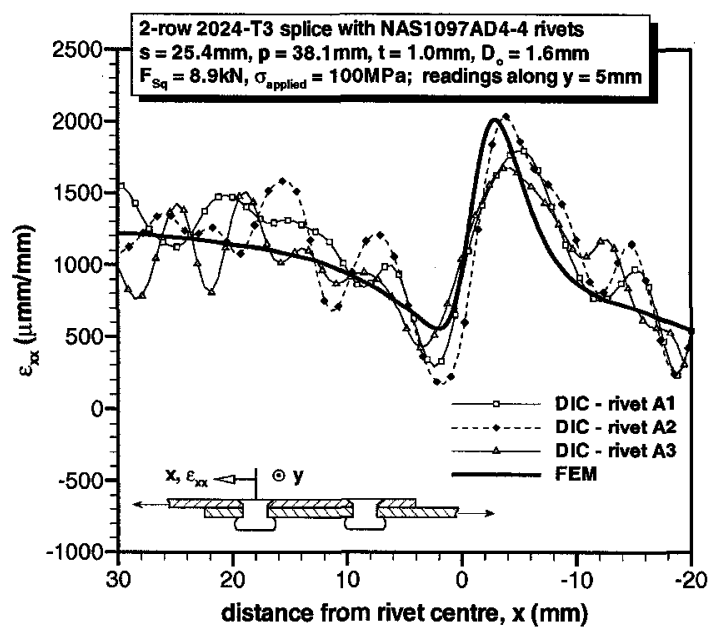

(a) longitudinal surface strain

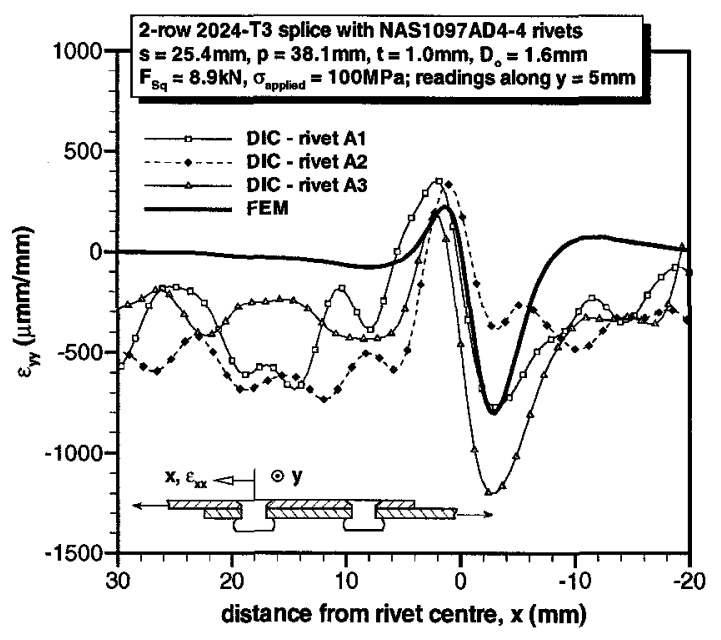

(b) transverse surface strain

Figure 5.3: Comparison of FE outer sheet surface strain results for a loaded lap joint to DIC experimental results

Based on the DIC results presented here, and the previous verification studies presented in Section 4.3.5, confidence in the performance and results of the splice loading finite element model has been established. 


\subsubsection{Secondary Bending Calculation}

The secondary bending factor, $K_{b}$, is not an explicitly determined quantity within the finite element code and requires additional post-processing to determine. As presented earlier in Chapter 2, the secondary bending factor is a ratio of the bending stress to the applied splice load $\left(\sigma_{\text {membrane }}\right)$ :

$$
K_{b}=\frac{\sigma_{\text {bending }}}{\sigma_{\text {membrane }}}
$$

This bending factor varies through the thickness of the sheet and is typically expressed for the faying joint surface where bending stresses are highest.

The concept of superposition is typically used to describe the resultant stress field in a loaded splice. The stress distribution through the thickness of the material consequently can be broken down into membrane and bending stress components as illustrated in Figure 5.4. As a result, the bending stress $\left(\sigma_{\text {bending }}\right)$ can be determined from the resultant faying and free surface stress components ( $\sigma_{\text {fay }}$ and $\sigma_{\text {free }}$ respectively) by:

$$
\sigma_{\text {bending }}=\frac{\sigma_{\text {fay }}-\sigma_{\text {free }}}{2}
$$

Within the current analysis we also have residual stresses present. Applying the concept of superposition in the presence of this residual stress field, however, would only be appropriate if the stress state was linear elastic. The residual stress field develops as a result of plasticity in the material and subsequent splice loading could produce further plasticity. If

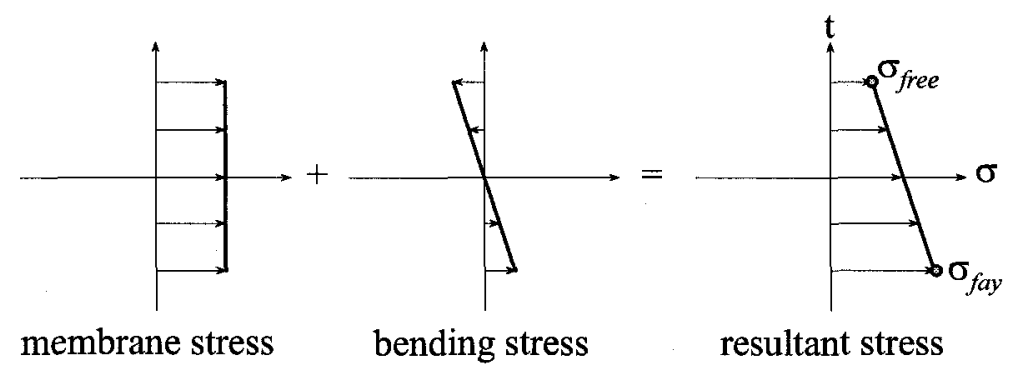

Figure 5.4: Application of superposition to resultant stress state in a splice under bending and membrane stresses 
superposition was used, the through-thickness stress distribution would be a combination of the membrane stress (linear distribution), bending stress (linear distribution) and residual stress (non-linear distribution). The result would be a non-linear stress distribution through the sheet thickness as sketched in Figure 5.5. This, however, was not observed in the FE simulations. The stress distribution through the thickness of the sheet was typically linear (Figures 5.6 and 5.7). Subtraction of the original residual stress state from these results would result in a non-linear distribution from which calculation of a bending factor would be inappropriate.

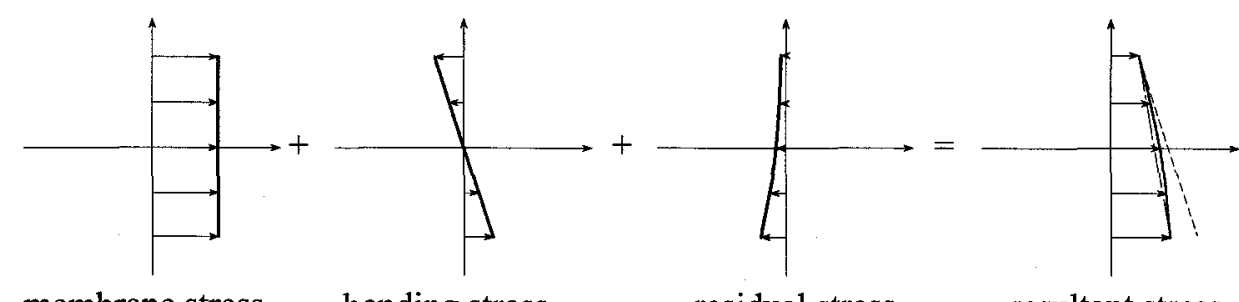

membrane stress

bending stress

residual stress

resultant stress

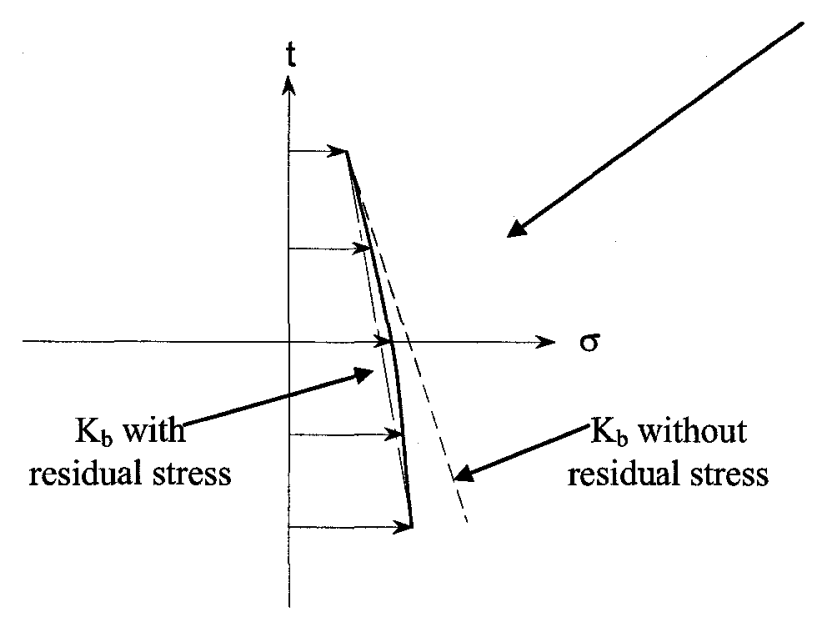

Figure 5.5: Resultant non-linear stress state in a splice when non-linear residual stress state is added using superposition

There were some regions in the joint where the through-thickness stress distribution was indeed non-linear (Figures 5.6 and 5.7). This non-linearity, however, is the result of tensile stresses due to bending exceeding the yield stress of the material along the faying joint surface. Subtraction of the original residual stress state from these regions did not result in a linear stress distribution. 

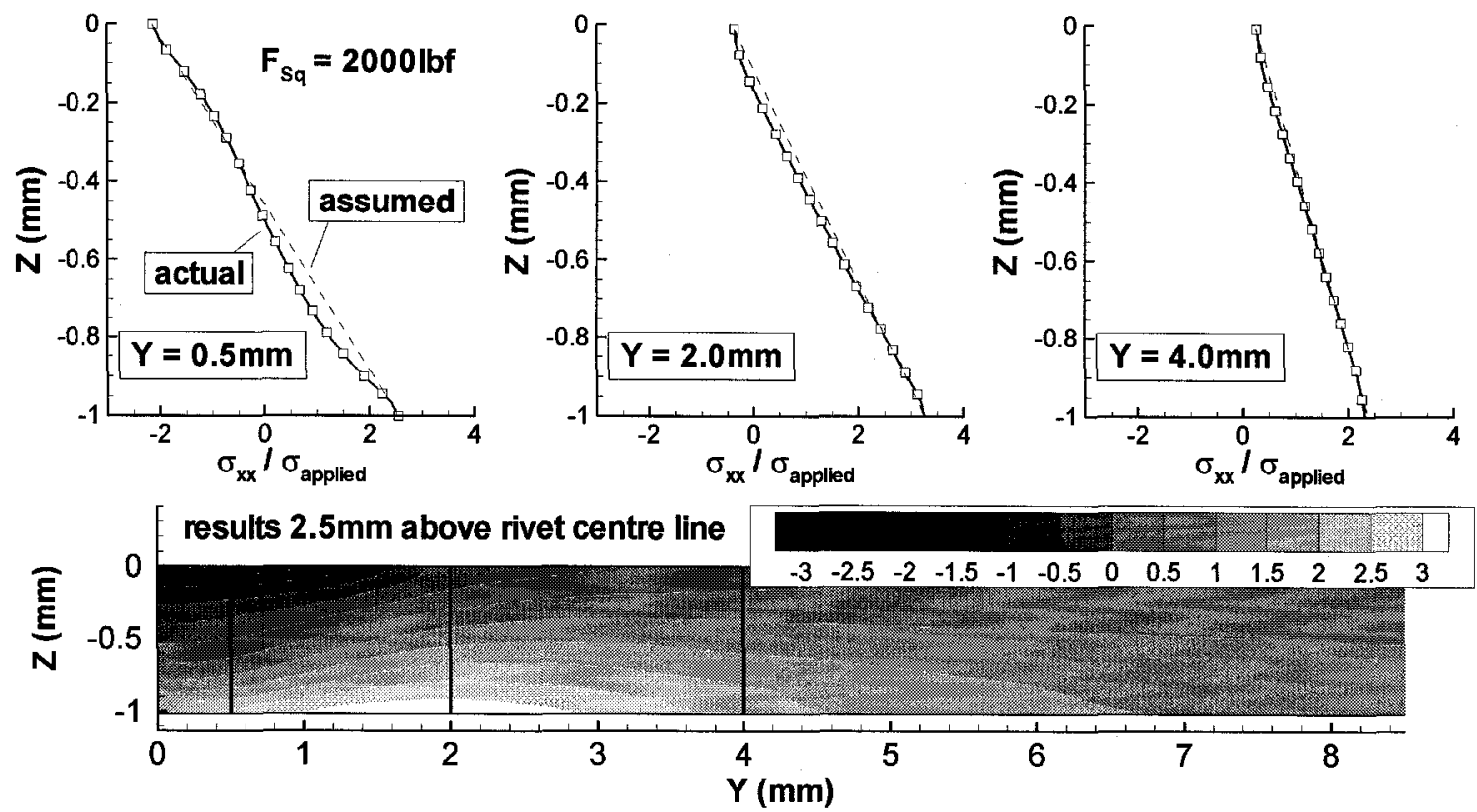

Figure 5.6: Outer sheet through-thickness stress distribution in a 2024-T3 universal splice $2.5 \mathrm{~mm}$ above the critical rivet row
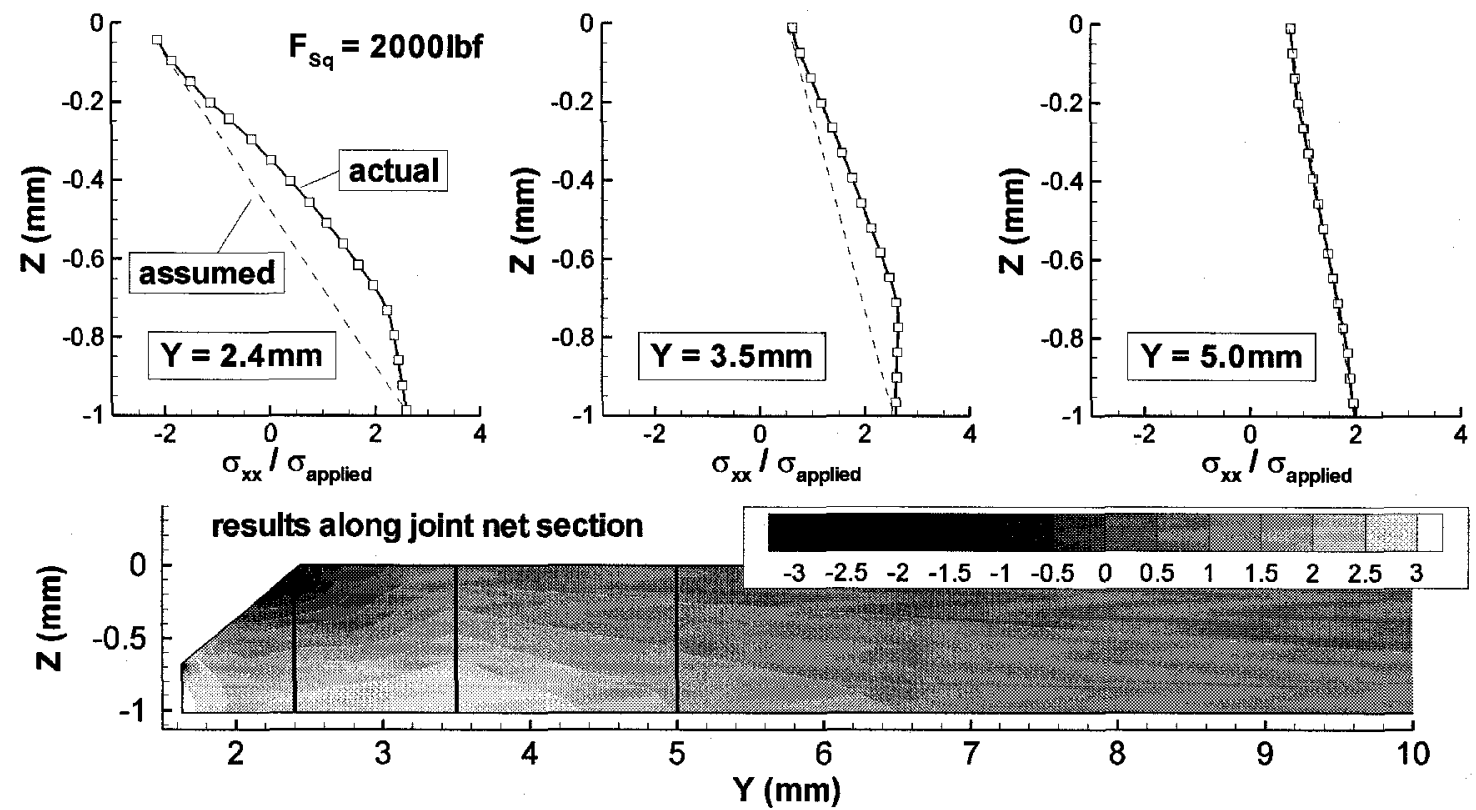

Figure 5.7: Outer sheet through-thickness stress distribution in a 2024-T3 countersunk splice along the net section of the critical rivet row 
Due to the above mentioned difficulties, the residual stress field was not removed from the overall stress field in order to determine $K_{b}$ from the finite element model. The bending stress, $\sigma_{\text {bending }}$, was determined using Equation 5.2 directly from the resultant stress field and used to calculate $K_{b}$ using Equation 5.1. Thus, the $K_{b}$ calculated from the finite element model includes residual stresses. The factor calculated in this work still represents the total bending observed in the loaded splice and can still be compared to predictions from secondary bending models. It must simply be noted that in regions of large variations in residual stress distribution, particularly within one rivet radius of the rivet hole, the calculated $K_{b}$ from this study deviates from its classical definition.

\subsection{Finite Element Results}

The findings reached during the present finite element study are discussed in the following section. Due to the volume of the results obtained, only subsets of the data are presented to support the discussed findings. In instances where the influence of the rivet squeeze force was observed to be small, results for $F_{S q}=2000 \mathrm{lbf}\left(D / D_{o}=1.5\right)$ and $3000 \mathrm{lbf}$ $\left(D / D_{o}=1.7\right)$ are used to illustrate the variation between a low and high squeeze force. Additional results collected in the study are available in Appendix D.

An additional coordinate frame was defined in order to facilitate comparison of the finite element results to the neutral line model. The global finite element coordinate frame, centred between the two rivet rows, is denoted by capital letters in Figure 5.8. The added coordinate frame, located along the rivet row centre line, is denoted by lower case letters. This additional coordinate frame allows the finite element and neutral line model results to be compared relative to the rivet row centre line.

As in previous chapters, the results will first be discussed in the context of the 2024-T3 simulations. Variations observed for the GLARE will be discussed in Section 5.4.3. 


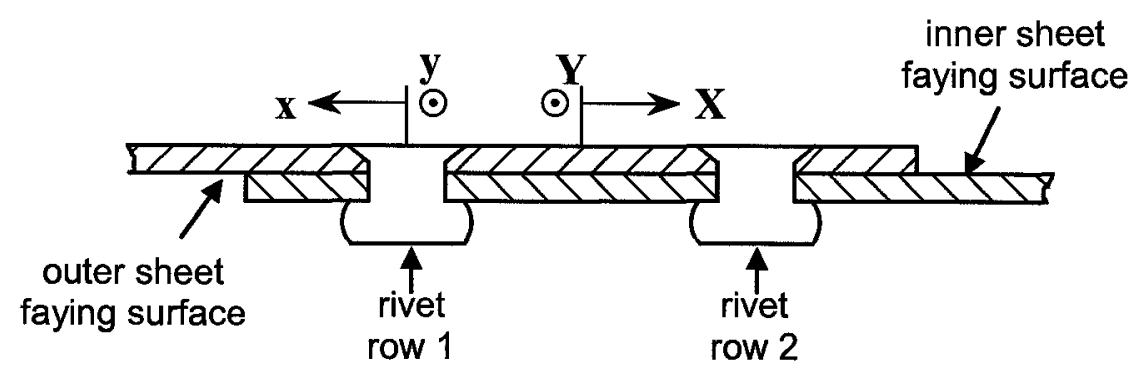

Figure 5.8: Illustration of coordinate systems used for presentation of $\mathrm{FE}$ results

\subsubsection{Neutral Line Model Comparison}

Representative contour plots of the secondary bending factor distribution are given in Figures 5.9 and 5.10 for the universal and countersunk rivet joint finite element models respectively. Due to limited variation in the results and for clarity, secondary bending results are only shown for the 2000 and $3000 \mathrm{lbf} F_{S q}$ cases. The limitations of the one-dimensional neutral line model for predicting secondary bending are clear from the variation in $K_{b}$ along the $\mathrm{X}$ and $\mathrm{Y}$ directions in these figures.

In order to provide a quantitative comparison to the neutral line model, selected results along the length of the joint from Figures 5.9 and 5.10 are compared directly to neutral

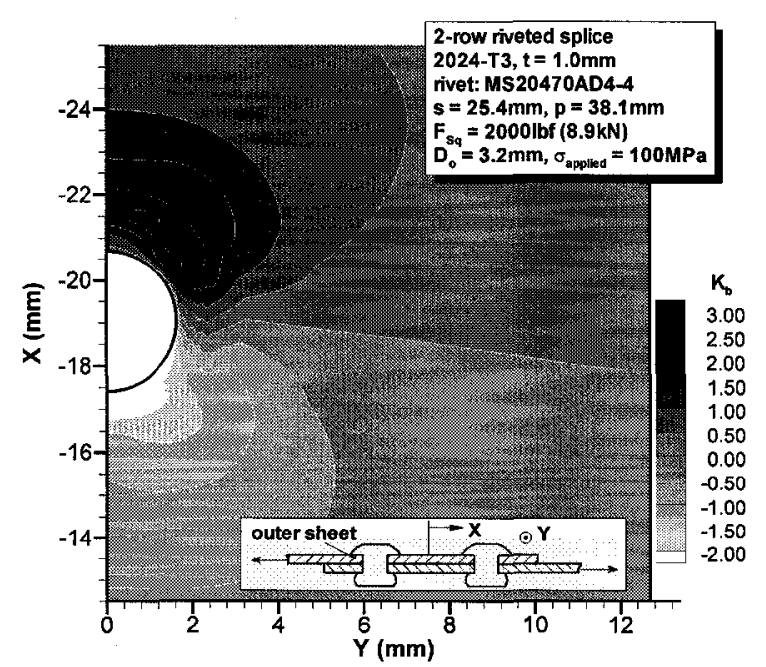

(a) $F_{S q}=2000 \mathrm{lbf}$

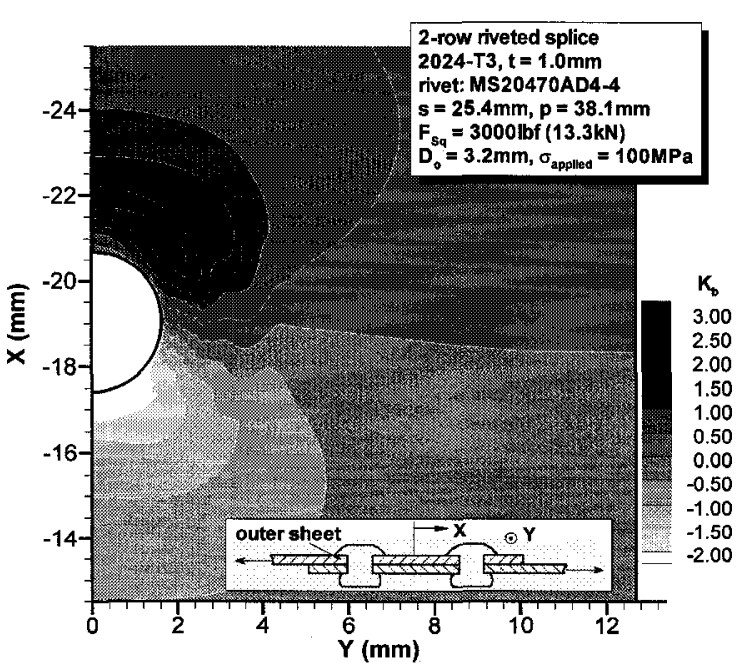

(b) $F_{S q}=3000 \mathrm{lbf}$

Figure 5.9: $\mathrm{FE} K_{b}$ contour results within the outer sheet of a universal rivet joint 


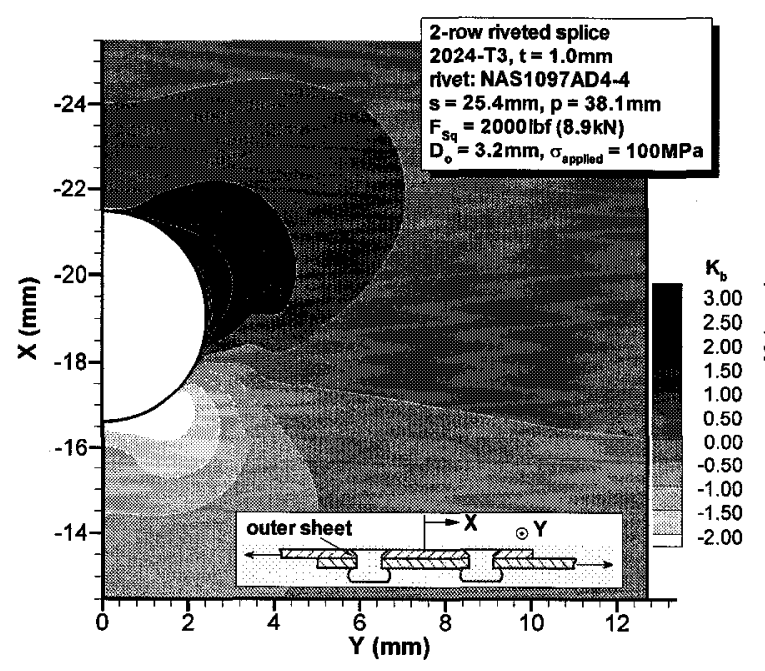

(a) $F_{S q}=2000 \mathrm{lbf}$

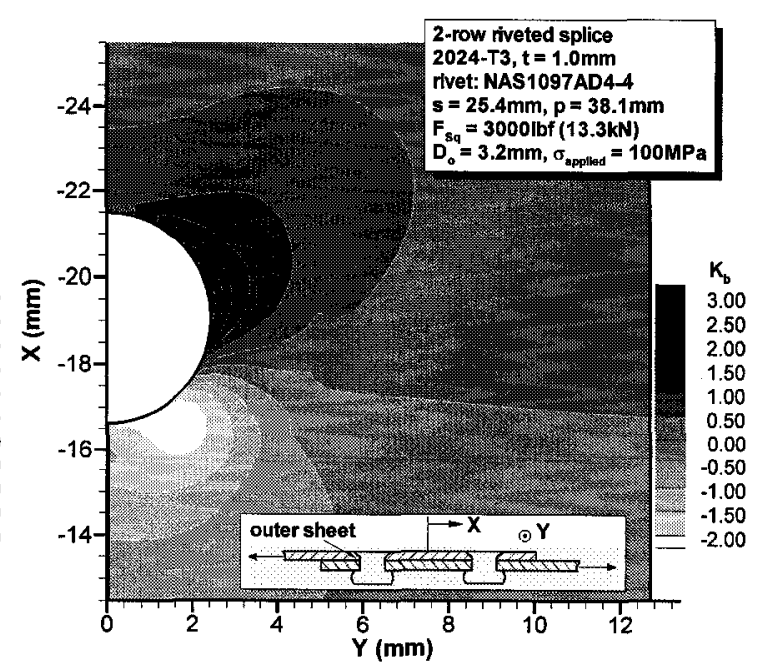

(b) $F_{S q}=3000 \mathrm{lbf}$

Figure 5.10: FE $K_{b}$ contour results within the outer sheet of a countersunk rivet joint

line model predictions in Figure 5.11. Figure 5.11a shows results obtained at two locations remote from the rivet hole while Figure $5.11 \mathrm{~b}$ shows results obtained near the rivet hole. At a distance of approximately $10 \mathrm{~mm}$ (3 rivet diameters) away from the rivet row centre line, variation in secondary bending along the joint width is small and the neutral line model provides an accurate prediction of $K_{b}$. From a fatigue analysis standpoint, agreement within this region is of little interest as crack nucleation and propagation occurs within one rivet diameter of the rivet row centre line. Within this region of interest, the neutral line model clearly results in under-predictions of $K_{b}$ near the rivet hole and over-predictions at locations between rivets.

Comparing the differences between the results from the countersunk and universal rivet joint splice models in Figure 5.11, it is clear that rivet type influences the distribution of $K_{b}$. Overall, the results for the universal rivet splice are similar to those of for the countersunk rivet splice, only shifted approximately $3 \mathrm{~mm}$ away from the rivet centre line. This is most easily seen by comparing the locations of peak $K_{b}$ in Figure 5.11a. This shift occurs due to the added constraint to rotation provided by the universal rivet head which causes peak secondary bending stresses to migrate away from the rivet hole. This concept was first illustrated in Figure 2.10 in Chapter 2. What these results contribute to this notion is that 


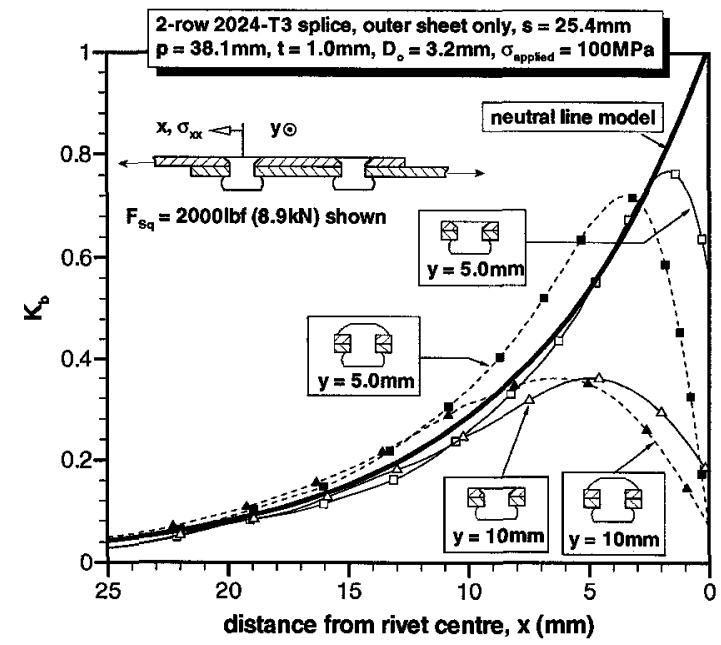

(a) remote from rivet hole (only $F_{S q}=2000 \mathrm{lbf}$ shown for clarity)

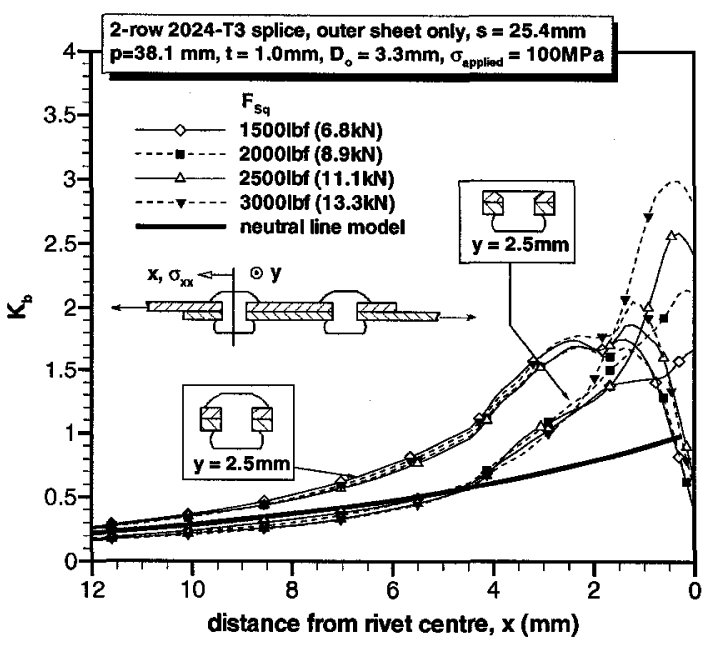

(b) near rivet hole

Figure 5.11: Comparison of $\mathrm{FE}$ outer sheet $K_{b}$ results to neutral line model predictions

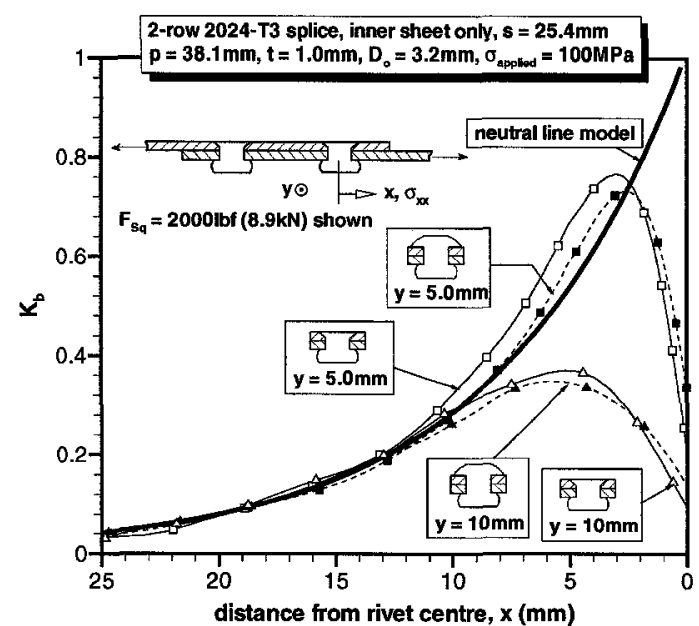

(a) remote from rivet hole (only $F_{S q}=2000 \mathrm{lbf}$ shown for clarity)

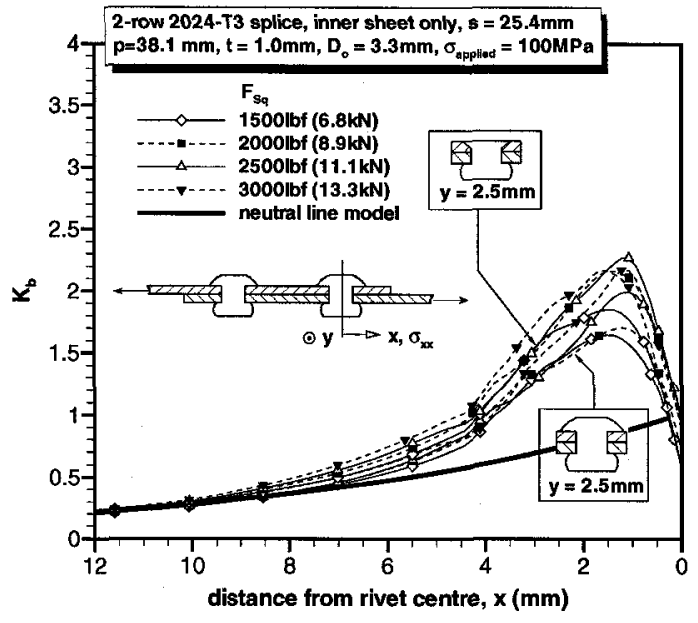

(b) near rivet hole

Figure 5.12: Comparison of $\mathrm{FE}$ inner sheet $K_{b}$ results to neutral line model predictions

this shift in secondary bending not only occurs at the rivet hole but is sustained away from the rivet as well.

Close to the rivet hole, as illustrated in Figure 5.11b, further deviation between the universal and countersunk rivet splice results occurs. The countersunk rivet splice model exhibits larger magnitudes of $K_{b}$ in comparison to the universal rivet splice. This is partially 
because the location of maximum $K_{b}$ migrates to the region above the rivet hole, and thus does not occur at the same location along the joint width compared to the countersunk rivet splice. Peak secondary bending stresses will be further discussed later in Section 5.4.2.

The influence of rivet type diminishes when considering the effect on $K_{b}$ in the inner joint sheet. This is evident in Figure 5.12 where selected finite element results for secondary bending in the inner sheet are plotted against the neutral line model. Within the inner sheet, the same shift in results observed for the outer sheet does not exist. This suggests that the geometry of the contacting rivet head (driven rivet head in the case of the inner sheet) has the dominant influence on secondary bending in a particular sheet. As the driven rivet head geometries are nearly identical for countersunk and universal rivets of the same diameter and length, and installed with the same squeeze force, it follows that the results for the two rivet types closely agree in Figure 5.12. Also, as the geometry of the driven rivet head more closely resembles that of the universal rivet head, it also follows that the results for the inner sheet resemble the outer sheet results for the universal rivet splice.

\subsubsection{Peak Secondary Bending}

The location and magnitude of peak secondary bending stresses predicted by the finite element models are shown in Figures 5.13 and 5.14 for the universal and countersunk rivet joint models respectively. Magnitudes are plotted in the main plot frames while the corresponding locations relative to the rivet hole are plotted in the inset frames. The constant peak secondary bending stress prediction produced by the neutral line model is also indicated for comparison purposes. Predicting the magnitude and location of peak secondary bending stresses is crucial for fatigue analysis as crack nucleation typically occurs in regions of high secondary bending. In this regard, the neutral line model grossly under predicts the magnitude of peak $K_{b}$ near the rivet hole, as was discussed in the previous section.

The finite element model results demonstrated a dependency between peak $K_{b}$ and both rivet type and $F_{S q}$. First, the dependency on $F_{S q}$ will be discussed. 


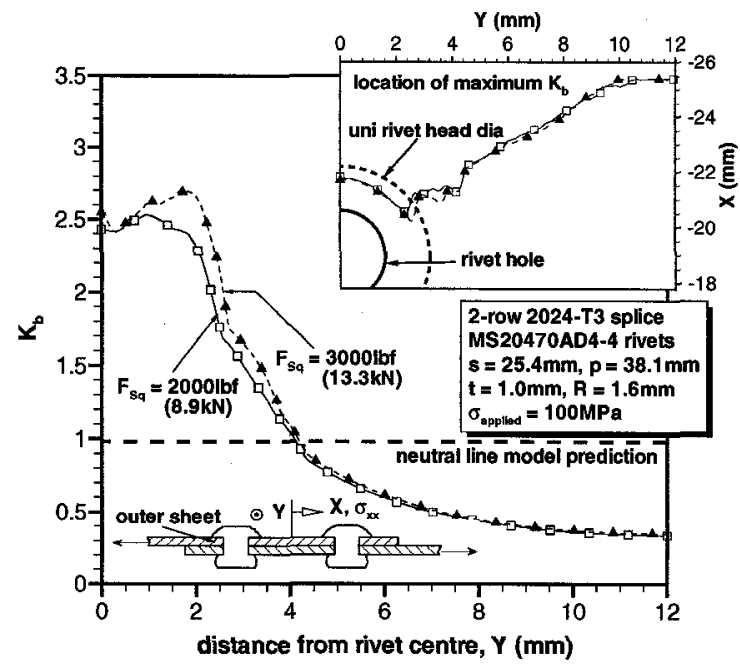

(a) outer sheet

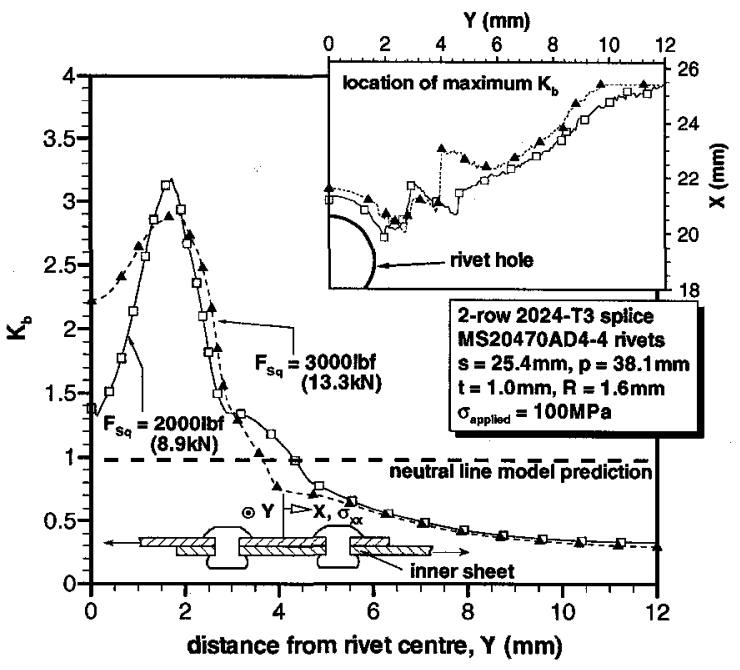

(b) inner sheet

Figure 5.13: Influence of $F_{S q}$ on the location and magnitude of maximum $K_{b}$ in a universal rivet joint

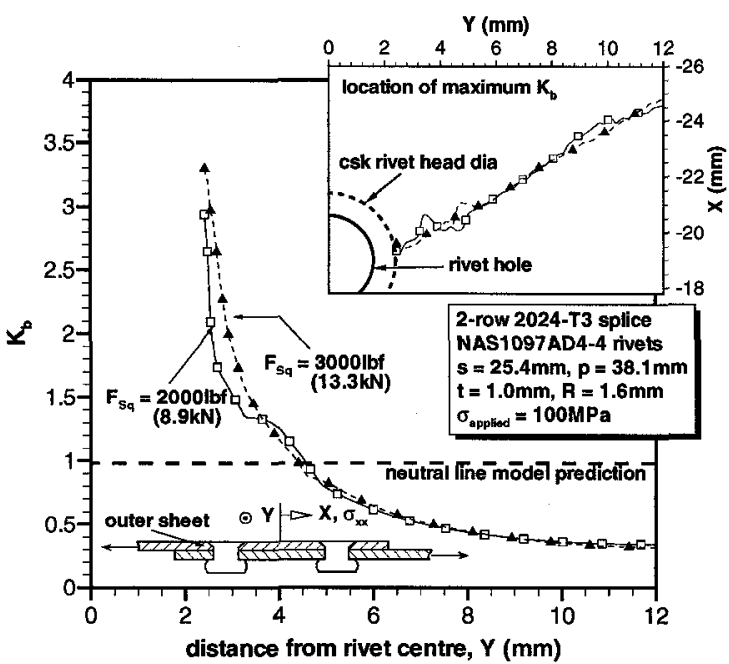

(a) outer sheet

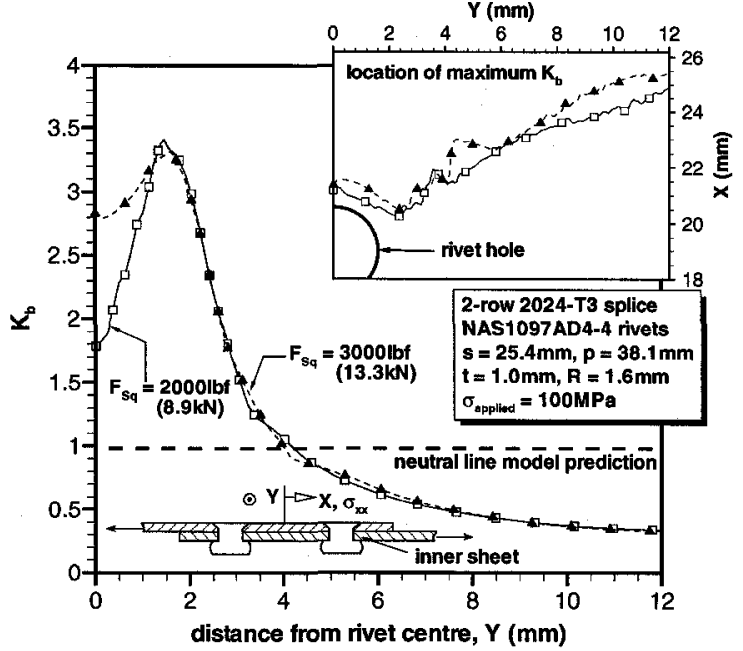

(b) inner sheet

Figure 5.14: Influence of $F_{S q}$ on the location and magnitude of maximum $K_{b}$ in a countersunk rivet joint 
Comparing the magnitudes of $K_{b}$ for the various $F_{S q}$ values plotted in Figures 5.13 and 5.14 , it is evident that these magnitudes are not strongly dependent on $F_{S q}$ for a given rivet type and sheet location. The largest deviations occur near the peaks of the graphs where differences are less than about $10 \%$ between the two $F_{S q}$ values shown in each plot.

The primary dependency on $F_{S q}$ occurs in the location of the peak $K_{b}$ values. Figures $5.13 \mathrm{~b}$ and $5.14 \mathrm{~b}$ show that the location of maximum $K_{b}$ in the vicinity of the rivet hole shifts further away from the hole by approximately $0.5 \mathrm{~mm}$ within the inner sheet. This distance also corresponds to the difference in the radius of the driven rivet head for the two $F_{S q}$ values plotted. Although this distance is small, it is not negligible as the residual stress field discussed in Chapter 4 can vary greatly over this distance. What is not observed in the finite element results is a shift in location of peak $K_{b}$ values within the outer sheet (Figures 5.13a and 5.14a). This indicates that the dependency on $F_{S q}$ is related to the geometry of the rivet heads. In the case of the outer sheet, the sheet is supported by the manufactured rivet head (countersunk or universal head) which does not change with $F_{S q}$. As a result, there is no shift in the location of peak $K_{b}$. Conversely, the inner sheet is supported by the driven rivet head which increases in size with $F_{S q}$ resulting in the observed shift.

A similar geometrical effect is observed when considering rivet type. To illustrate, results for $F_{S q}=2000 \mathrm{lbf}$ from Figures 5.13 and 5.14 have been combined in Figure 5.15. The universal rivet provides a large head adjacent to the outer sheet which provides greater constraint to rivet rotation than the countersunk type rivet. This results in the shift of location of maximum $K_{b}$ from the rivet centre line to above the rivet hole as shown in Figure 5.15a. As the geometry of the driven rivet heads is dependent on $F_{S q}$ and not rivet type, no shift is observed in Figure 5.15b.

The importance of the shift in the location of maximum $K_{b}$ observed in the outer sheet for the two rivet types becomes apparent when considering the influence of residual stress. For cracks that nucleate and grow along the rivet centre line, the residual tangential stress component is critical as it is perpendicular to the cracking direction. For cracks that nucleate in the region of maximum secondary bending above the rivet hole, the radial stress component becomes more critical. Given that crack nucleation typically occurs in this 


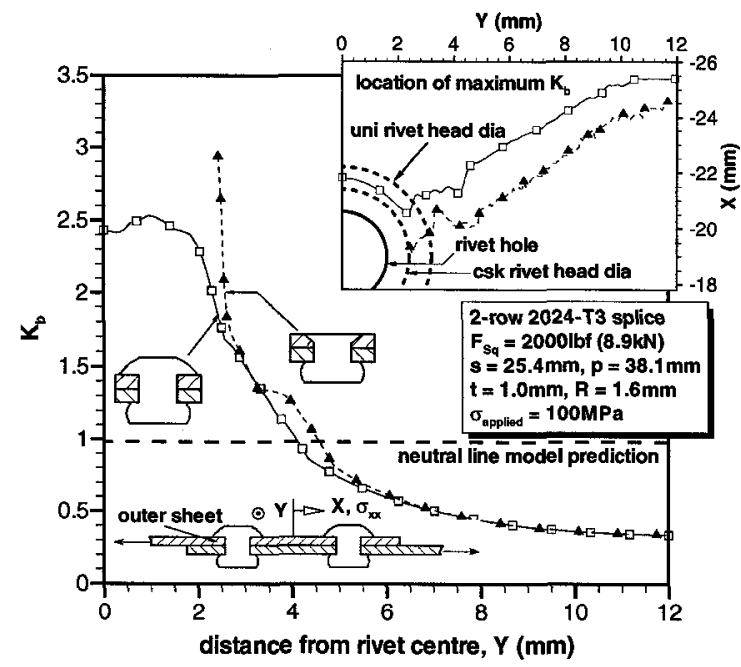

(a) outer sheet

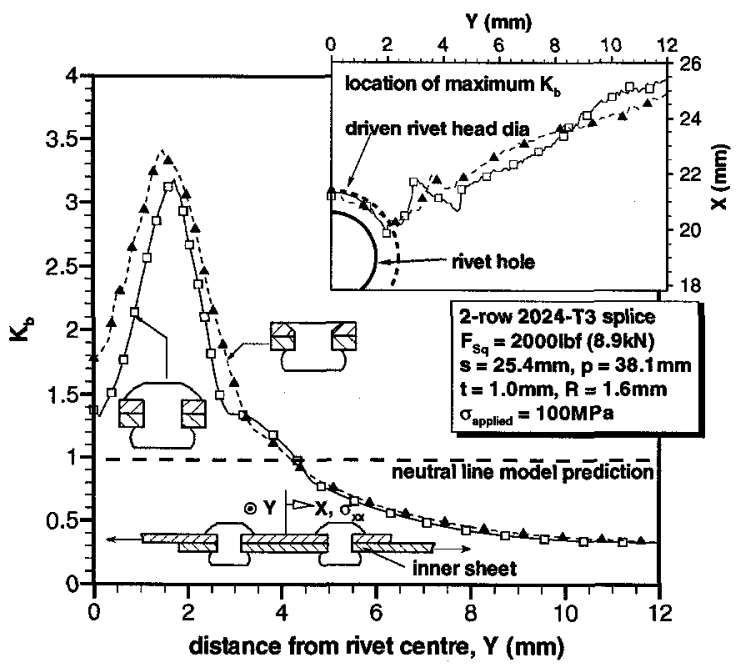

(b) inner sheet

Figure 5.15: Comparison of location and magnitude of maximum $K_{b}$ in universal and countersunk rivet joints $\left(F_{S q}=2000 \mathrm{lbf}\right.$ shown)

region of high secondary bending, the benefits of the residual stress field may be dependant on the type of rivets used.

\subsubsection{Influence of Sheet Material}

Overall, similar trends were observed for the GLARE3-2/1-0.3 riveted joint models as described in the previous sections for the 2024-T3 riveted joint models. Significant differences and unique features observed in the GLARE models will be described in this section.

When comparing the GLARE and 2024-T3 joint model results, sheet thickness needs to be kept in mind. The nominal thickness of GLARE3-2/1-0.3 is $0.86 \mathrm{~mm}$, which was used in the finite element models. The closest gauge of 2024-T3 sheet that is commercially available is $1.0 \mathrm{~mm}$, which was also used in the finite element models. The effect of this difference is two-fold. First, the joint eccentricity present in the GLARE joint models is smaller due to the smaller sheet thickness. Second, since the models were simulated using an applied tensile stress of $100 \mathrm{MPa}$, the resultant load per unit width for the GLARE joint model is lower than that for the 2024-T3 model. These effects alter the driving forces for secondary bending for the two sheet materials, making direct comparison of their results difficult. 
Selected results along the length of the GLARE3-2/1-0.3 joint model are compared with neutral line model predictions in Figure 5.16. One feature stands out in the GLARE results in comparison to the 2024-T3 results shown in Figures 5.11 and 5.12. The countersunk joint model predicts a higher peak magnitude of $K_{b}$ in comparison to the universal joint model for GLARE sheet. This is a result of the lower stiffness of the GLARE sheet material. The lower stiffness of GLARE results in larger deformation of the sheet, and thus elongation of the rivet hole, during loading. The increased elongation of the rivet hole reduces the constraint provided by the countersunk rivet head, permitting increased rivet rotation. This added rivet rotation in turn increases the degree of secondary bending.

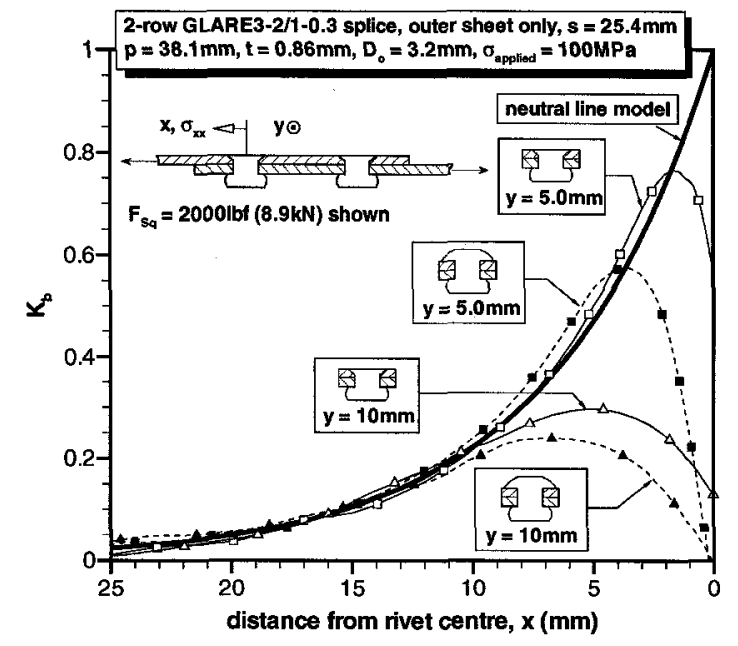

(a) outer sheet

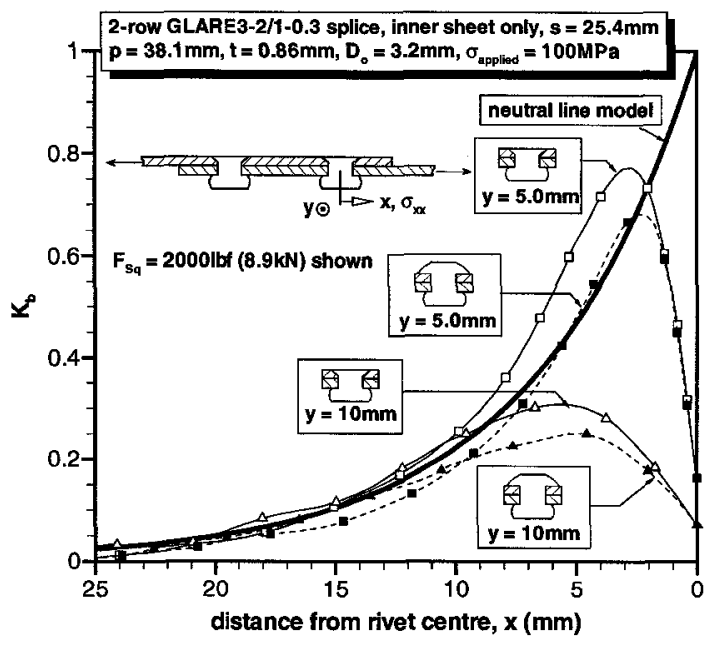

(b) inner sheet

Figure 5.16: Comparison of FE $K_{b}$ results for GLARE3-2/1-0.3 sheet to neutral line model predictions

The presence of this added rivet rotation for countersunk GLARE joints was also observed in fatigue tests carried out by Brown [54]. In two-row lap joint fatigue tests on coupons made of GLARE3-2/1-0.3 and 2024-T3, the GLARE coupons were found to fail due to rivet fatigue and eventually rivet pull-through rather than crack nucleation and eventual fracture of the sheet material. Furthermore, these failures occurred before the fracture failures in the 2024-T3 coupons, indicating that the rivet rotation causing the failure had to be more severe in the case of the GLARE coupons. 


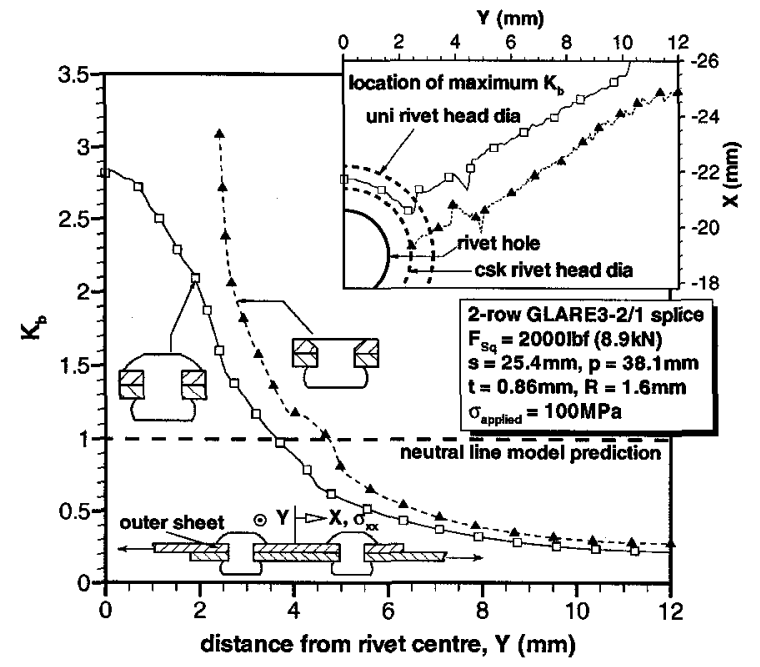

(a) outer sheet

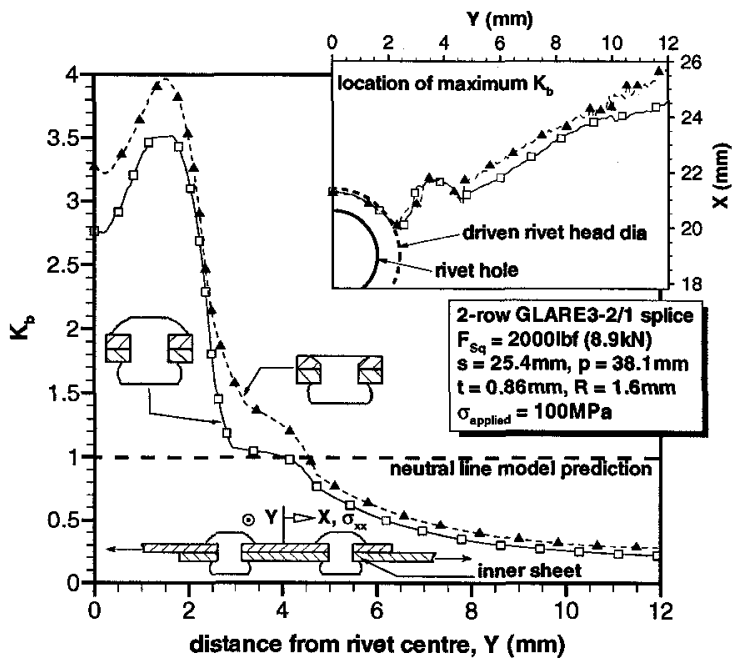

(b) inner sheet

Figure 5.17: Comparison of location and magnitude of maximum $K_{b}$ in universal and countersunk GLARE3-2/1-0.3 rivet joints $\left(F_{S q}=2000 \mathrm{lbf}\right.$ shown $)$

The effects of this added rivet rotation are also seen in plots of the peak $K_{b}$ observed along the width of the joint as shown in Figure 5.17. Higher magnitudes of $K_{b}$ are observed in the countersunk rivet case as a result of the added rivet rotation. This is observed in both the outer and inner sheet (Figures $5.17 \mathrm{a}$ and $\mathrm{b}$ respectively), however, it is more pronounced in the outer sheet where the reduced constraint of the countersunk rivet head exacerbates the effect.

Despite differences in magnitudes of $K_{b}$ between the 2024-T3 and GLARE3-2/1-0.3 joint models, the locations of the peak $K_{b}$ values along the width of the joint correlate well (compare the inset plots in Figures 5.15 and 5.17). Since the geometry of the driven and manufactured rivet heads is near identical for a given rivet squeeze force between the 2024-T3 and GLARE joint models, this reinforces the link between the location of secondary bending stresses and rivet head geometry discussed in the previous sections. 


\subsection{Conclusions}

The uniaxial loading of a two-row riveted lap joint has been simulated using a 3-dimensional finite element analysis. The primary goal of this analysis was to study the role of rivet type and installation on secondary bending in both 2024-T3 and GLARE3-2/1-0.3 riveted lap joints. Based on the results of this analysis, the following conclusions can be made:

- The neutral line model provides accurate predictions of secondary bending at a distance of three rivet diameters or greater from the rivet row centre line. Within three rivet diameters, significant variation in secondary bending was observed along the joint width, making the one-dimensional neutral line model inaccurate.

- The neutral line model underestimates the amount of secondary bending present near the rivet hole where crack nucleation occurs.

- The location and magnitude of peak secondary bending is affected by the geometry of the constraining rivet head. For bending within the outer sheet, this constraint is provided by the manufactured rivet head for which the geometry is dictated by rivet type. For bending in the inner sheet, this constraint is provided by the driven rivet head for which the geometry is influenced by the rivet squeeze force.

- Within the outer sheet, peak secondary bending stresses flow around the rivet hole with the largest magnitudes occurring above the rivet hole for a universal rivet joint. For a countersunk rivet joint they intersect with the rivet hole with the largest magnitudes occurring at the hole edge. As these represent likely locations for crack nucleation, the benefit of the riveting induced residual stress state on crack growth may vary for the two rivet types.

- The magnitude of secondary bending stresses near the rivet hole is larger for countersunk rivet joints in comparison to universal rivet joints. Remote from the rivet hole, the two rivet types produce similar results. 
- The higher flexibility of GLARE sheet compared to 2024-T3 sheet resulted in larger rivet rotations in the countersunk rivet joint models. Higher rivet rotations resulted in increased secondary bending. These higher rivet rotations were not observed in the universal rivet joint models due to the added constraint of the universal rivet.

Overall, the results of this study highlighted some of the major deficiencies in the 1dimensional neutral line model, supporting the need for a model which accounts for the discrete location of individual fasteners in a riveted joint, and identified the level of constraint provided by fastener heads as the primary factor affecting the location and magnitude of secondary bending stresses.

The previous two chapters have focused on quantifying the influence of rivet installation and geometry on the residual stress state and on secondary bending stresses in a riveted lap joint using finite element methods. In the following chapter, the effects of these stresses on crack nucleation and growth will be examined. 


\section{Chapter 6}

\section{Crack Growth}

\subsection{Introduction}

Knowledge of how the stress state in a riveted lap joint is influenced by riveting is of no use for design against fatigue if the effects of this stress state on crack nucleation and growth are not known. This chapter seeks to fill this remaining gap by presenting an experimental study undertaken to quantify the influence of rivet installation on crack nucleation and growth. Unlike in previous chapters, this chapter only considers the effects of rivet installation on the fatigue performance of monolithic 2024-T3 aluminum sheet. Some of the experimental methods employed were not suitable for GLARE laminates, thus GLARE laminates had to be excluded from the study. This exclusion is further discussed in Section 6.3.5.

\subsection{Review of Crack Growth in Riveted Splices}

Conventional crack growth measurement techniques typically involve the use of optical trav-

elling microscopes to measure the progression of visible surface crack tips. Such techniques are often used in conjunction with non-destructive inspection techniques such as eddy current or ultrasound to facilitate the detection of sub-surface or small surface cracks and to establish a time-to-detection life for fatigue damage. This general approach is well suited to investigations relating to practical damage tolerance assessments, where the behaviour of easily detectable fatigue cracks is of interest. 
Studying the effects of riveting induced residual stresses and secondary bending on crack nucleation and growth requires consideration of several additional factors. The region of beneficial residual stresses is localized around the rivet hole and is obstructed by the rivet head, reducing the effectiveness of conventional crack detection and measurement techniques. Without direct crack growth measurements in this region, only qualitative assessments of the effects of residual stresses can be obtained from time-to-detection lives. These issues were first discussed in Section 4.2 in regard to experimental determination of the residual stresses, but they apply equally to experimental crack growth measurement.

Secondary bending further complicates the study of crack nucleation and growth. The secondary bending stresses resulting from rivet rotation slow the through-thickness progression of a part-through crack. As a result, it is possible for a part-through crack to grow through most of the region affected by residual stress and peak secondary bending stresses before it develops into a visually detectable through-crack. Furthermore, the propagation rate of the crack front along the joint width varies with thickness location as a result of secondary bending. Simple measurements of the length at the visible surface are not sufficient for quantifying this effect.

Fractography offers a more comprehensive way to quantify crack growth behaviour [5558]. Post-failure fractographic analyses of a fracture surface, typically using a scanning electron microscope (SEM), yield results for crack growth of part-through cracks, provide information on crack front shape and its evolution, and can be used to identify the location of crack nucleation. Crack growth information can be obtained from fractographic analysis using two methods. The first method involves determining the spacing of individual fatigue striations [55-57]. From this spacing, the crack growth rate at that point can be determined. This method provides accurate measurements of growth rate at discrete points on the fracture surface but does not aid in the visualization of crack fronts.

The second method involves identifying striation patterns or groupings of striations resulting from variations in loading within the fatigue spectrum. These variations in loading can be obtained from patterns of distinct loads in the case of a part subjected to a variable amplitude load, or can be introduced into a constant amplitude spectrum with periodic over- 
or under-loads. Such introduced loads are often referred to as marker loads. By identifying the striation patterns associated with marker loads, the location and shape of the crack front can be identified. If sufficient information on the fatigue spectrum is available, crack growth rates between these patterns can be estimated. The primary drawback to the use of marker loads to estimate crack growth is determining the effect of the over- or under-load on crack growth. Large over-loads can result in an increase in the crack tip plastic zone which influences the growth rate of subsequent base-line cycles. Similarly, for under-load cycles, the baseline cycles can influence the growth during the marker loads. Schijve [58] provides a further detailed description of the effects of over- and under-loads on crack growth and fractographic reconstructions.

Both the striation spacing and marker load methods of fractographic crack growth reconstruction have been employed in previous studies of mechanically fastened joints. The effects of cold expansion induced residual stresses on crack growth in open-hole 2024-T3 Alclad specimens was investigated by de Matos et al. [55] using the striation spacing method of fractographic crack growth reconstruction. By means of the fractographic reconstruction, de Matos et al. were able to identify that retardation of early crack growth by the compressive residual stresses provided the bulk of the improvement in fatigue life for cold expanded holes; an improvement once believed to be the result of delayed crack nucleation. The use of striation spacing was also employed by Moreira et al. [59] to investigate crack growth in a 3-row riveted lap joint. Using the concept of equivalent initial flaw size (EIFS), Moreira et al. demonstrated that fatigue life predictions for a general loading case could be made based on fractographic reconstruction data from a single load case.

Several studies have also successfully employed fractographic reconstructions using a marker load fatigue spectrum. Piasick and Willard [60] developed a unique marker load spectrum (Figure 6.1) to investigate crack fronts obtained in riveted lap joints which incorporated marker bands in groupings of 10,4 , and 6 bands. The 10-4-6 repetitive groupings aid in the reconstructions, providing a means to identify if a particular marker is missed. Fawaz [51] and de Rijck [2] both used the 10-4-6 marker band spectrum to investigate crack growth in open-hole tension and tension-bending specimens. The primary goal of these 


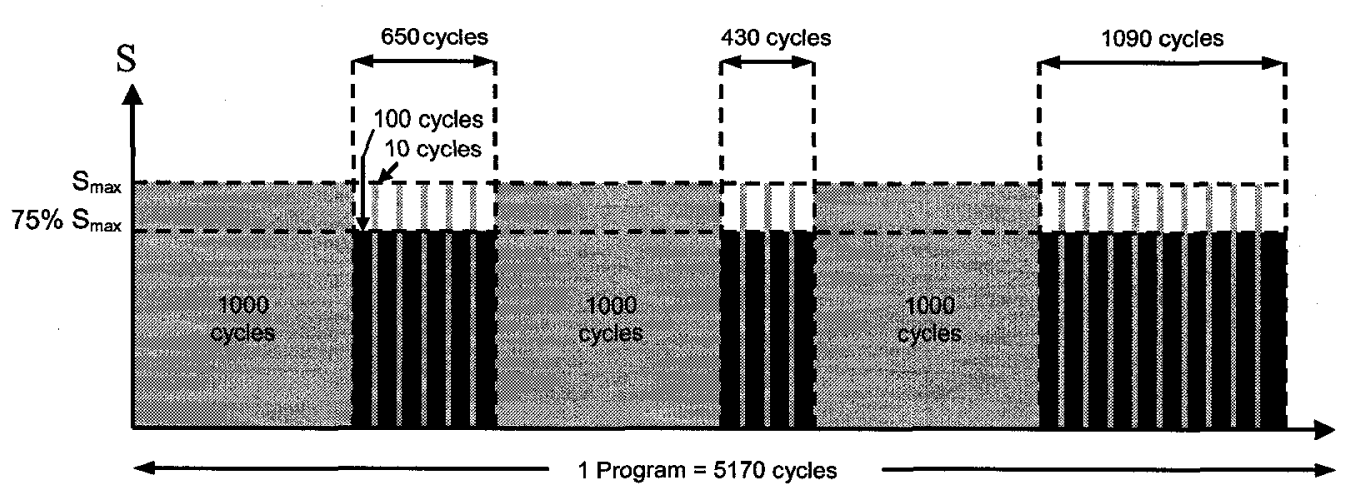

Figure 6.1: Schematic of 10-6-4 marker-band spectrum [60]

investigations was to determine crack front shape as a function of length in order to develop improved estimates of stress intensity factors for riveted joints.

Despite the extensive use of fractographic reconstructions to investigate the fatigue performance of mechanically fastened joints, none have considered the effects of rivet installation. Given the results of the previous chapters related to the effects of rivet type and installation on residual stresses and secondary bending, the application of fractographic methods to investigate these effects on crack growth would provide a valuable contribution to the field. The development of and results obtained from an experimental fractographic reconstruction study using the 10-4-6 marker band spectrum will be presented in the following sections.

\subsection{Fatigue Crack Growth Tests}

Two sets of fatigue tests were carried out in order to investigate the effects of rivet installation on the fatigue performance of riveted lap joints. The first set was undertaken to quantify the effects of rivet squeeze force and rivet type on overall fatigue life. The second set was designated for fractographic crack growth reconstruction. Due to the time consuming nature of such reconstructions, these tests were not run until fracture. Rather, once cracks of a given size were present, the tests were stopped and the specimen disassembled for fractographic analysis. 


\subsubsection{Test Specimen Geometry}

Fatigue tests were conducted on 2-row riveted lap joint specimens similar in configuration to the 2-row lap joint modelled in Chapter 5. The specimen geometry is detailed in Figure 6.2. Specimens were manufactured with 2024-T3 Alclad sheet and 2117-T4 rivets. Alclad refers to a thin layer of pure aluminum placed on the sheets for corrosion resistance. It is important to note that this Alclad layer was not considered in the finite element analysis detailed in the previous chapters. Specimens containing countersunk (NAS1097AD4-4) and universal (MS20426AD4-4) rivets were made. As mentioned previously, GLARE specimens were not tested.

The orientation of the rivets in the two rivet rows was reversed giving a true antisymmetric geometry. This modification, although not practical in a commercial application, is useful for experimental investigations as it ensures the same conditions are present in the two fatigue critical locations (Section 2.4.1). This anti-symmetric configuration results in two specimen types, designated type 1 and type 2 (Figure 6.2). For the type 1 configuration, crack nucleation is assured to occur next to a manufactured rivet head (countersunk or universal rivet head), simulating an outer sheet failure. For specimen type 2, an inner sheet

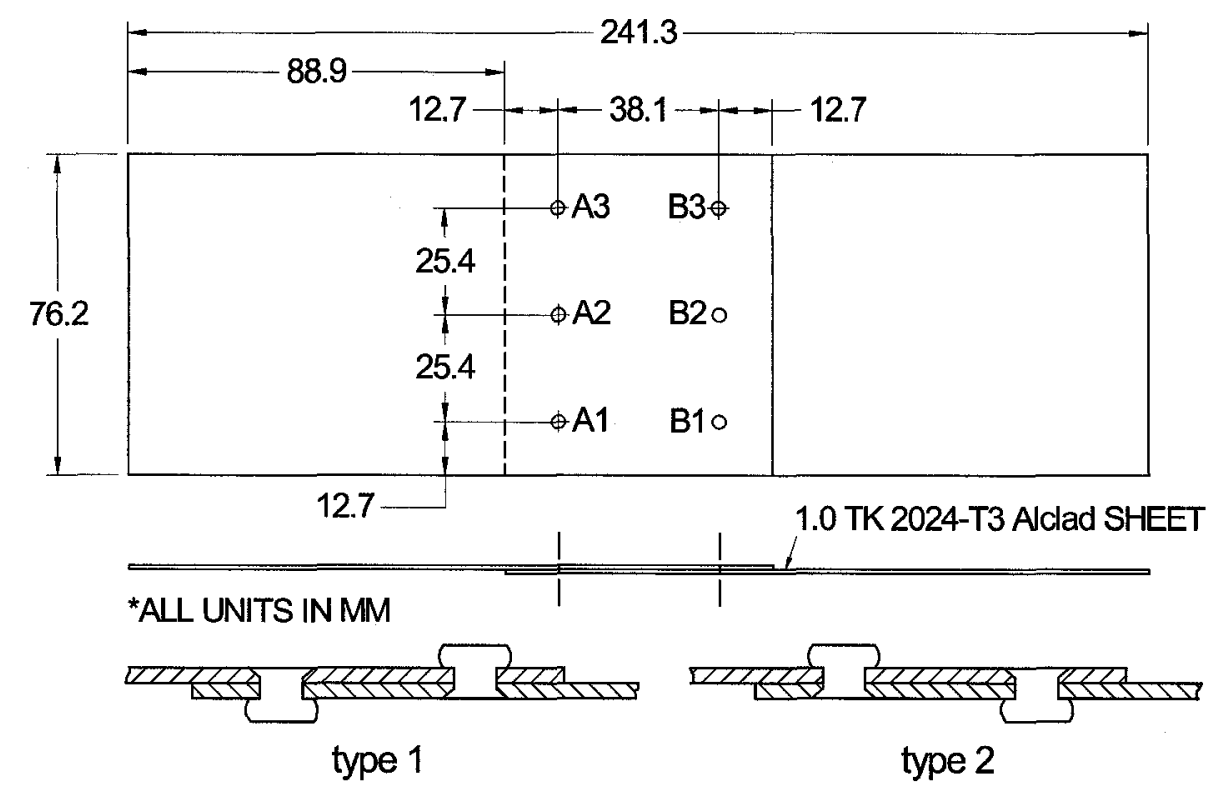

Figure 6.2: Fatigue specimen geometry (adapted from coupon design presented in [1]) 
failure is ensured. Use of these two specimen types permits the effects of rivet installation on inner and outer sheet failure to be studied independently.

\subsubsection{Fatigue Test Spectrum}

To permit crack growth reconstruction, the 10-4-6 marker band spectrum used in previous studies $[2,51,60]$ was adopted for this investigation. Based on four preliminary crack growth reconstruction trials, it was found that the number of cycles in each block needed to be modified for the crack growth rates present in this investigation. The modified spectrum is shown in Figure 6.3.

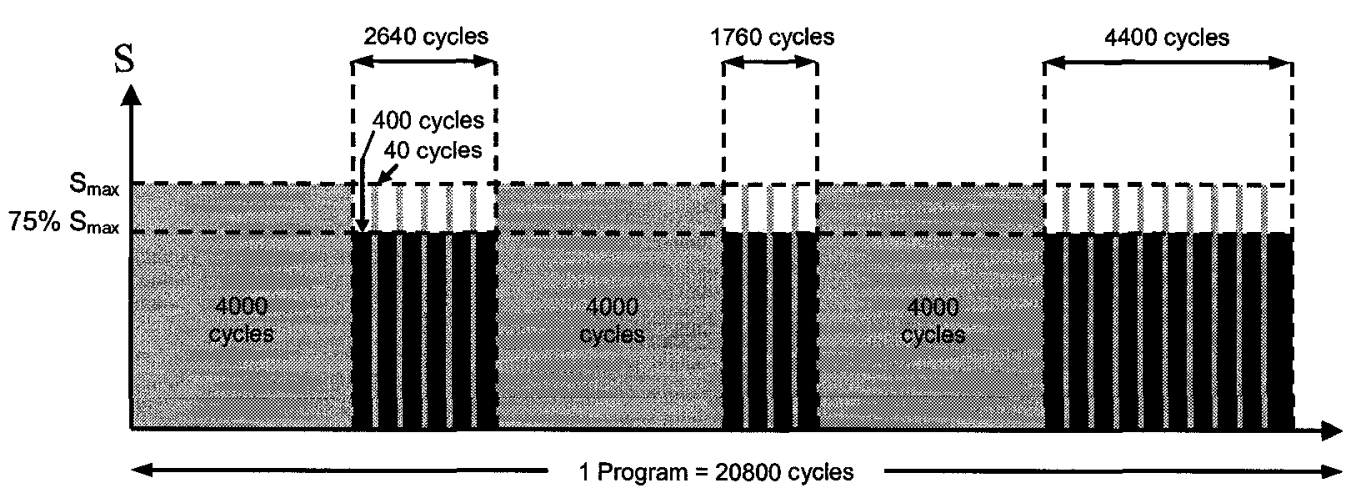

Figure 6.3: Schematic of modified 10-6-4 marker-band spectrum

A maximum stress level, $S_{\max }$, of $100 \mathrm{MPa}$ was specified, corresponding to the same maximum stress level simulated in Chapter 5. To prevent damage of the marker bands on the fracture surface, a stress ratio $R=S_{\max } / S_{\min }=0.1$ was specified to minimize contact between the opposing fracture surfaces during unloading. All tests were carried out at a frequency of $10 \mathrm{~Hz}$ on an MTS 318.10 load frame equipped with a $10 \mathrm{kN}$ load cell and a TestStar IIs ${ }^{\mathrm{TM}}$ controller.

Although the marker band spectrum was only required for the set of tests designated for crack growth reconstructions, the spectrum was used in all tests. To account for the difference in crack growth at the $75 \% S_{\text {max }}$ cycles, a correction factor derived using Elber's plasticity induced crack closure concept [61] was used. This approach was developed by 
Fawaz [51] and applied in addition studies completed by de Rijck [2]. For the loading case used in this investigation, de Rijck [2] determined that the ratio of the crack growth rate for the $75 \% S_{\max }$ and $S_{\max }$ cycles is $9.22 \%$ in $2024-\mathrm{T} 3$ and verified this for open-hole fatigue specimens. For the derivation of this factor, the reader is encouraged to refer to the work of de Rijck [2].

Given the $9.22 \%$ crack growth correction factor, one program of the marker band fatigue spectrum, which consists of 20800 cycles, reduces to 13538 baseline cycles. This correction factor is based on differences in crack growth and does not necessarily account for differences in crack nucleation between the baseline and marker load cycles. As a result, verification tests were undertaken to see how well the overall fatigue life of specimens tested with a constant amplitude spectrum correlated with specimens tested with the marker band spectrum and corrected using the above correction factor. The results of this study are shown in Figure 6.4. An ANalysis Of VAriance (ANOVA) [62] study was performed on the two groups of data resulting in a $\mathrm{P}$-value of 0.911 , indicating that there is less than a $10 \%$ chance that the two groups of results represent distinct populations. Based on this result, and the verification studies performed by de Rijck [2], the correction factor was deemed sufficient for correcting both crack growth and nucleation results for the present study. As such, all fatigue lives and crack growth rates will be presented with the applied correction factor unless otherwise noted.

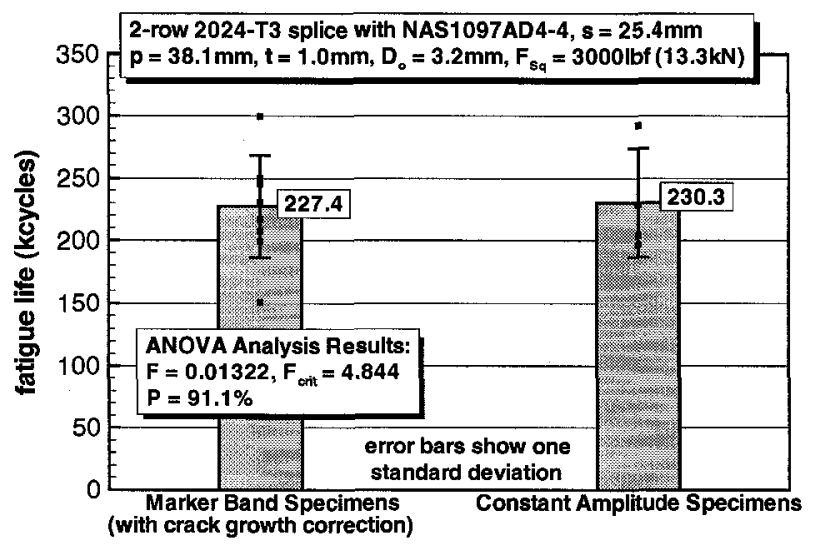

Figure 6.4: Comparison of fatigue lives for coupons tested with and without the marker band spectrum 


\subsubsection{Test Matrix}

Two series of fatigue tests were undertaken in this investigation: a series to determine overall fatigue life, and a series for fractographic crack growth reconstruction (designated $F$ and $S E M$ respectively). Furthermore, two specimen configurations (Type 1 and 2 in Figure 6.2) and specimens containing two rivet types were tested. In order to distinguish between the various specimen configurations and test types undertaken, the following specimen naming convention was used:

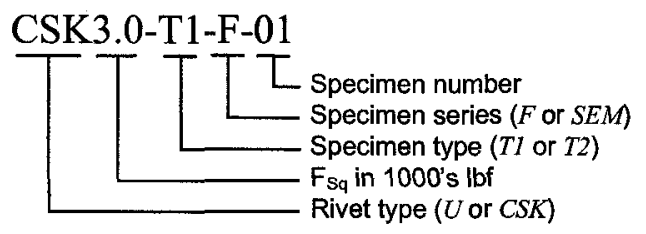

Where the specimen series designators $F$ and $S E M$ refer to the overall fatigue life and fractographic reconstruction series respectively. The specimen type designators refer to the type numbers illustrated in Figure 6.2 and the rivet type designators of $U$ and $C S K$ refer to the rivet types MS20426AD4-4 and NAS1097AD4-4 respectively. All specimens were made with 2024-T3 Alclad sheet.

All of the tests completed are summarized in the test matrix given in Table 6.1. There are a lesser number of tests conducted on Type 2 specimens as these tests were performed only to verify that the outer sheet failure which occurs in the Type 1 specimens is more critical. Additionally, as difficulties were encountered with fractographic reconstructions for specimens containing universal type rivets (MS20426AD4-4), a larger number of tests were conducted on countersunk rivet (NAS1097AD4-4) specimens.

\subsubsection{Crack Growth Reconstruction}

Crack growth reconstructions were performed using a JEOL JSM-6400 scanning electron microscope. The reconstruction procedure was as follows. A photographic survey of the fracture surface was taken in the SEM facility at 450x magnification, with a probe voltage of $20 \mathrm{kV}$ and a working distance of $21 \mathrm{~mm}$. The resulting images from the survey were manually assembled to generate a single high resolution image of the fracture surface. From 
Table 6.1: Test matrix for 10-4-6 marker band fatigue tests

\begin{tabular}{ccccc}
\hline$F_{S q}(l b f)$ & Rivet Type & Specimen Type & No. F-Specimens & No. SEM-Specimens \\
\hline 1500 & NAS1097-AD4-4 & 1 & 6 & - \\
2000 & NAS1097-AD4-4 & 1 & 6 & 3 \\
2500 & NAS1097-AD4-4 & 1 & 10 & - \\
3000 & NAS1097-AD4-4 & 1 & 9 & 3 \\
\hline 1500 & MS20426-AD4-4 & 1 & 4 & - \\
2000 & MS20426-AD4-4 & 1 & 4 & 1 \\
2500 & MS20426-AD4-4 & 1 & 4 & - \\
3000 & MS20426-AD4-4 & 1 & 4 & 1 \\
\hline 1500 & NAS1097-AD4-4 & 2 & 3 & - \\
2000 & NAS1097-AD4-4 & 2 & 3 & - \\
2500 & NAS1097-AD4-4 & 2 & 3 & - \\
3000 & NAS1097-AD4-4 & 2 & 3 & - \\
\hline 1500 & MS20426-AD4-4 & 2 & 3 & - \\
2000 & MS20426-AD4-4 & 2 & 3 & - \\
2500 & MS20426-AD4-4 & 2 & 2 & - \\
3000 & MS20426-AD4-4 & 2 & 2 & - \\
\hline
\end{tabular}

this high resolution composite image, the locations of the advancing crack front could be identified from the pattern of 10-, 4-, and 6-band markers visible on the fracture surface (Figure 6.5). Due to the smaller striation spacing, blocks containing the lower load cycles $\left(75 \% S_{\max }\right)$ show up as dark lines or featureless bands at the given magnification. Once all the crack fronts were identified, the life of the specimen for each front could be determined by identifying the interface between fatigue and static failure (associated with the final life of the specimen, see Figure 6.5) and counting backwards in blocks of cycles for each crack front. In some instances, it was difficult to detect particular crack fronts; however, it was easy to determine and account for such missing fronts due to the alternating 10-4-6 marker band pattern expected. Final crack growth measurements were made relative to the hole edge as observed on the fracture surface (Figure 6.6). For cases where cracks did not intersect the rivet hole, crack front positions were measured relative to the rivet centre line. 


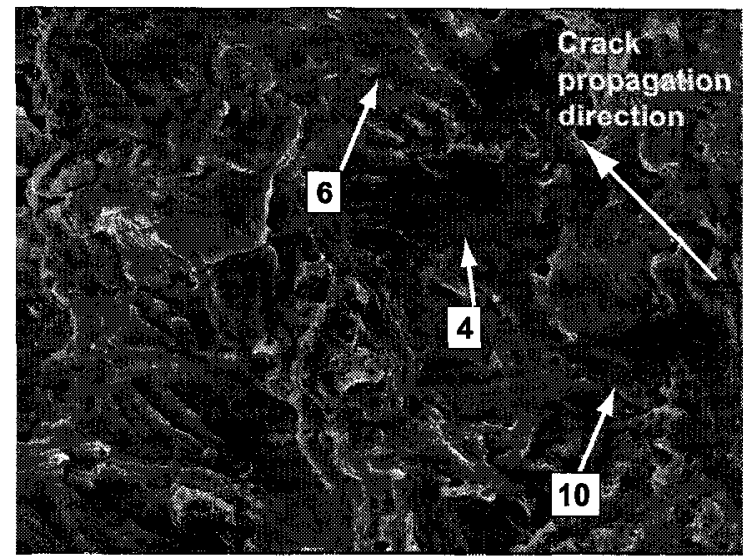

(a) visible marker bands

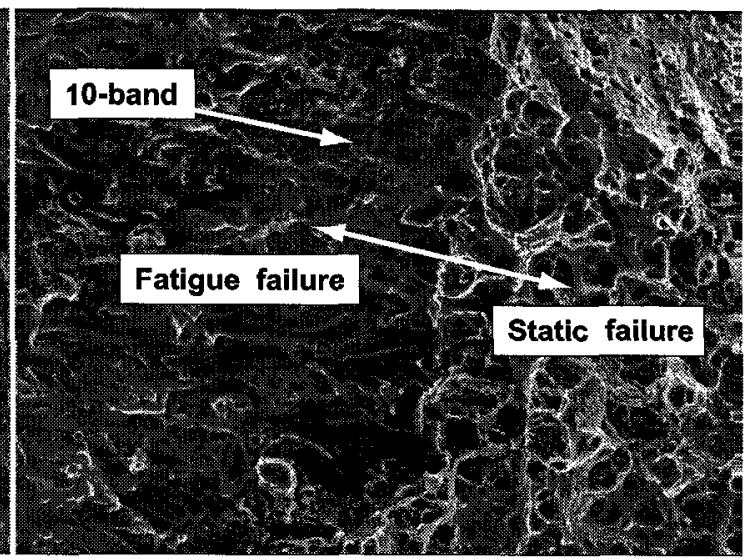

(b) fatigue-static failure interface

Figure 6.5: Examples of SEM reconstruction features
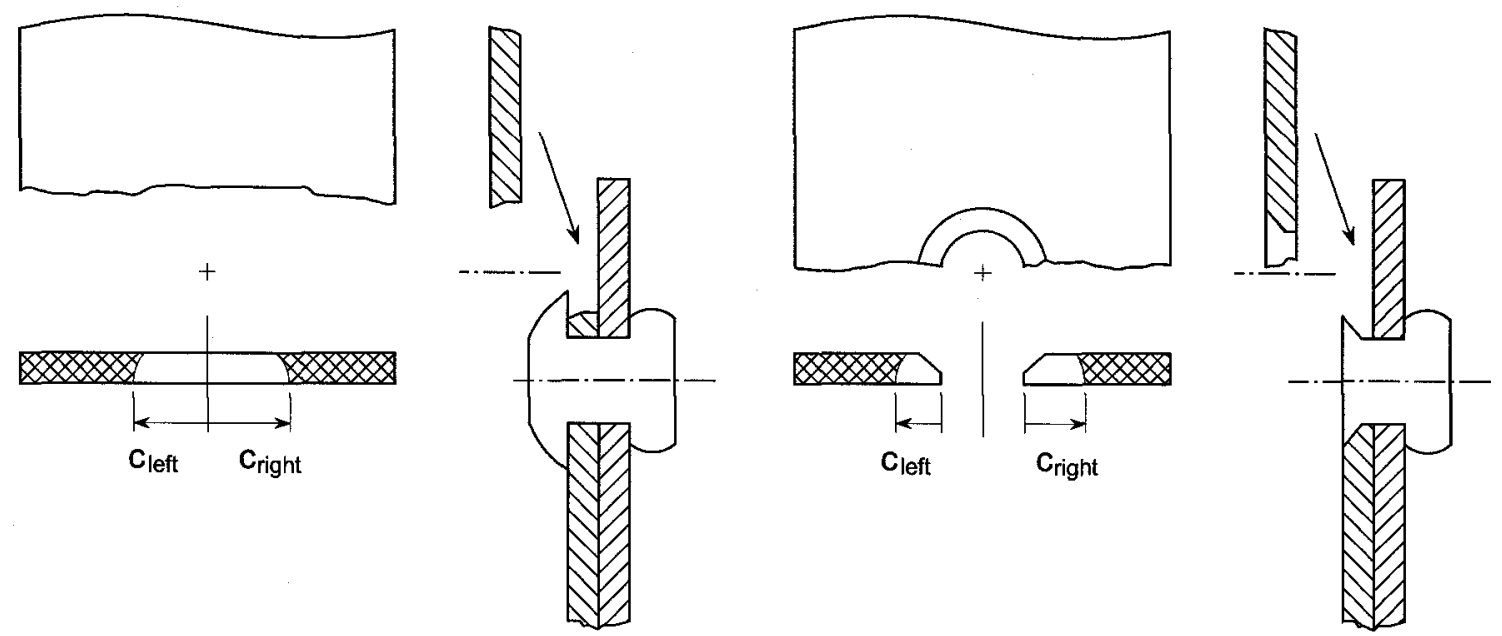

Figure 6.6: Crack measurement datums

Several factors limited the extent to which the crack growth reconstructions could be performed. First, as crack length increases, the morphology of the fracture surface changes. At large crack lengths, the fracture surface becomes dominated by micro voids which impede the detection of the marker bands. Second, the reconstruction process requires many hours to complete. For both these reasons, testing of fatigue specimens destined for crack growth reconstruction were stopped once a crack of approximately $2-3 \mathrm{~mm}$ was visible. As each specimen contained 6 rivet locations where cracking could occur, the rivet containing the largest cracks was always selected for crack growth reconstruction. 


\subsubsection{GLARE}

Testing of GLARE riveted lap joints was excluded from this study for a number of reasons. First and foremost, the fractographic reconstruction method being employed in this study is not well suited for GLARE laminates. In order to identify marker location during a reconstruction, an unobstructed view of the fracture surface is necessary. Fracture of the fibre layer, however, does not provide a clean fracture surface. Stray and bent fibres from the fractured fibre layer block the line of sight to the aluminum layer fracture surfaces in the SEM. It is possible to stop a fatigue test prior to failure of the fibre layer while still having cracks present in the aluminum layers. However, in order to observe the aluminum layer fracture surfaces, the aluminum layers would need to be removed from the fibre layers. Unfortunately, methods for separating the aluminum and fibre layers in GLARE typically do so at the cost of destroying or severely altering the aluminum layers, compromising the fracture surface. For this reason, crack growth reconstructions could not be performed on GLARE specimens.

Although crack growth reconstructions could not be performed, it was feasible to fatigue test GLARE specimens in order to obtain overall fatigue life data. In light of the absence of crack reconstruction data, however, such tests were deemed not to provide any substantial contribution to the present study. Such fatigue life results are already available in the work of Müller [1]. Consequently, no fatigue tests were performed on any GLARE specimens and efforts were focused on reconstructions and fatigue tests of 2024-T3 specimens.

Even though tests were not done on GLARE specimens, some of the expected behaviour of GLARE can be inferred from the 2024-T3 results. Residual stresses and secondary bending will have similar influences on fatigue of the aluminum layers within GLARE as it will on monolithic aluminum sheet. These stresses have been quantified for both materials in the previous chapters. Thus, the 2024-T3 results should allow conclusions to be drawn on both materials. 


\subsection{Overall Fatigue Life Results}

The present experimental study comprised of two series of fatigue tests. Results from the first series, which included fatigue tests to quantify overall fatigue life, are presented in the following section. Results from the second series, which included fractographic reconstructions of crack growth, are presented in Section 6.5.

The overall fatigue life results for both specimen configurations (Type I and II) manufactured with the universal and countersunk rivet types are shown in Figure 6.7. Type I and II specimen configurations correspond to outer sheet and inner sheet failure respectively. As different population sizes were used to determine the mean fatigue life, statistical confidence intervals based on the standard deviation and population size are included. A detailed breakdown of the coupon results and determination of the confidence intervals can be found in Appendix E.

Inner sheet failures are undesirable in airframe skin joints as access to the inner surface of the fuselage is necessary during inspections to detect cracks. For this reason, only a limited number of tests were performed on the Type II specimen configuration with the primary objective of establishing if inner or outer sheet failure would likely occur first for

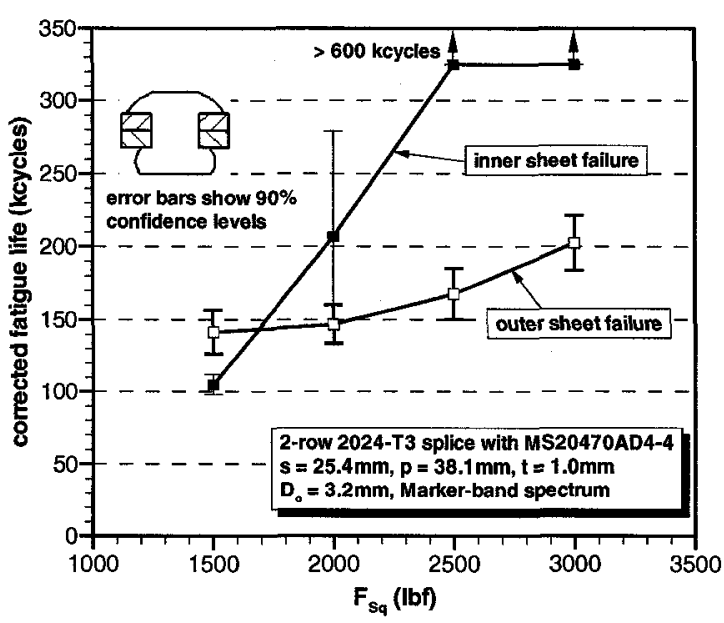

(a) universal joint

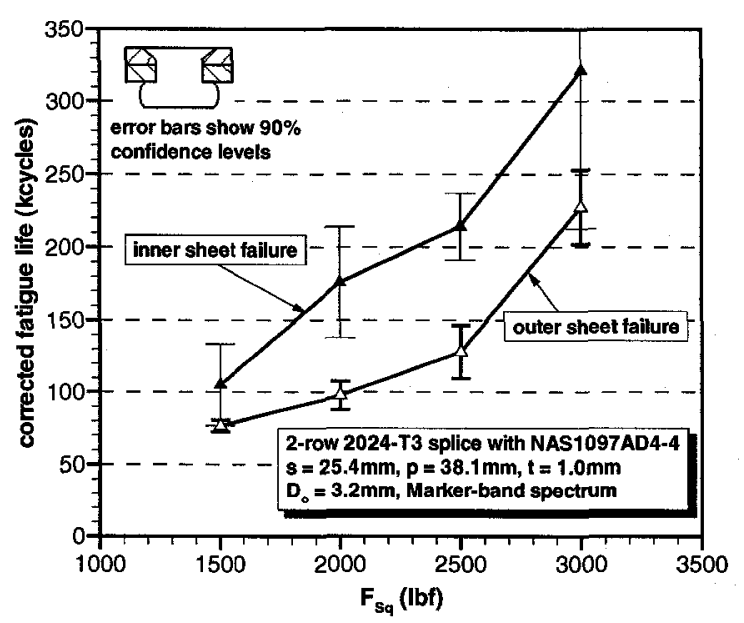

(b) countersunk joint

Figure 6.7: Overall fatigue lives for inner and outer sheet failure 
a given rivet squeeze force. Outer sheet failure was favoured for rivet squeeze forces equal to or greater than $2000 \mathrm{lbf}$ for both rivet types. At high squeeze forces, the disparity in compressive residual stresses between the inner and outer sheet promote failure in the outer sheet. At lower squeeze forces, this disparity is reduced and the probability of failure in the inner sheet increases. This was observed in the universal rivet specimens where inner sheet failure became critical for the lowest squeeze force examined. At this squeeze force, outer sheet failure remained critical for the countersunk rivet specimens as a result of the added stress concentration provided by the machined countersink.

The overall fatigue life results for outer sheet failure are re-plotted in Figure 6.8 in order to facilitate comparison of the universal and countersunk rivet specimen results. Although both the universal and countersunk rivet specimens displayed a rising trend in fatigue life with increasing rivet squeeze force, some distinct features in the results were observed for the two rivet types.

For the universal rivet specimens, a small increase in mean fatigue life was observed with increasing squeeze force. Taking into account the scatter observed in the tests (indicated by the $90 \%$ confidence interval bars in Figure 6.8), however, the mean fatigue lives observed for squeeze forces below $3000 \mathrm{lbf}$ are not statistically distinct according to the ANOVA

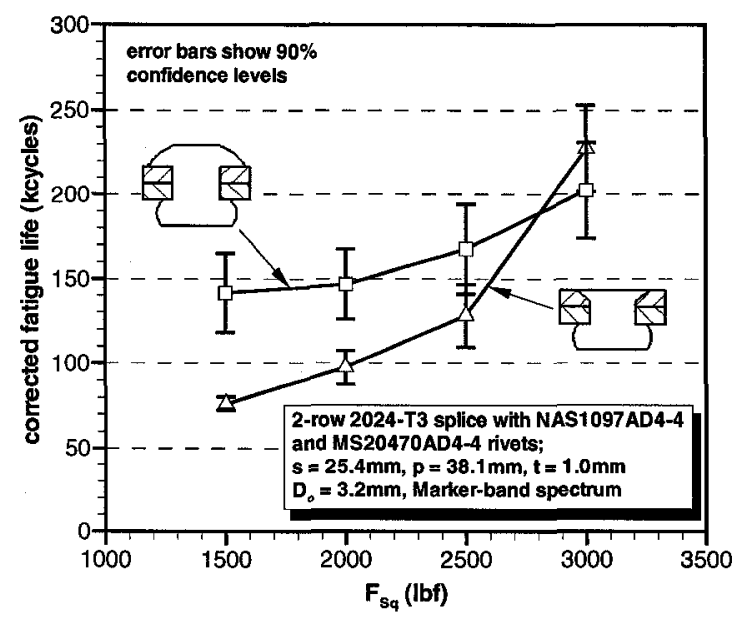

Figure 6.8: Comparison of overall fatigue lives for universal and countersunk joints (outer sheet failure only) 
analysis detailed in appendix E. This relative insensitivity of overall fatigue life to rivet squeeze force results from the location of fatigue damage observed in the universal rivet specimens as shown in Figure 6.9. Fatigue cracks nucleated above the rivet hole and grew around it. This location corresponds to the region of maximum secondary bending stress observed for universal rivet joints in Chapter 5 and marked in the Figure. This location for crack nucleation and growth is also beyond the region of beneficial residual stress generated for the majority of rivet squeeze forces examined (indicated by the plastic zone radii in Figure 6.9). This explains why a statistically distinct increase in fatigue life was only observed once the squeeze force became large enough to induce a beneficial residual stress state at the location of cracking.

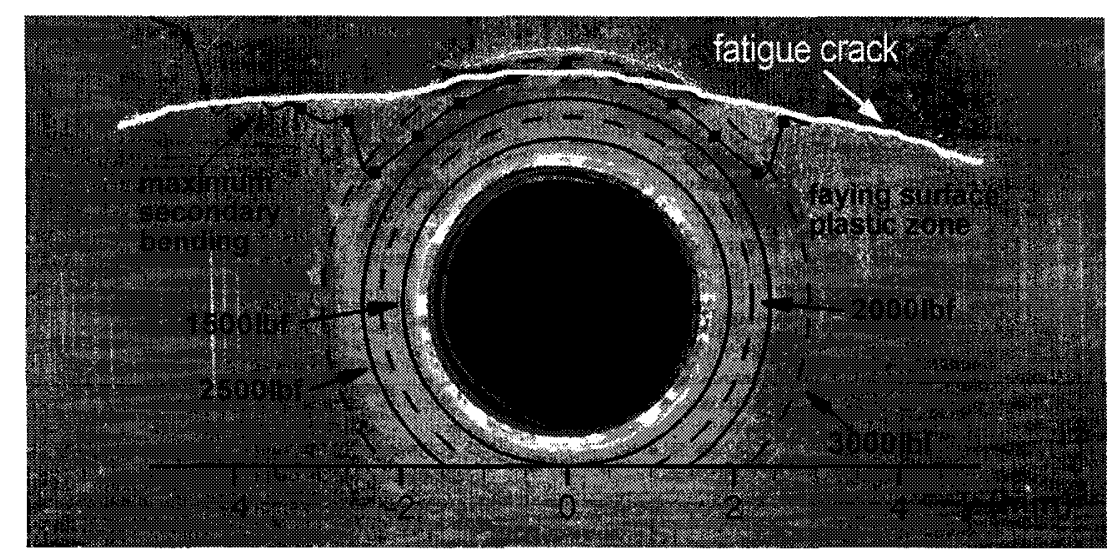

Figure 6.9: Typical location of fatigue damage observed in universal rivet joint fatigue specimens with $\mathrm{FE}$ outer sheet faying surface plastic zone radii

Conversely, the results for the countersunk rivet specimens show a strong dependency between squeeze force and overall fatigue life. In contrast to the universal rivet specimens, fatigue damage occurs close to the rivet row centre line as shown in Figure 6.10. Cracks nucleate at the hole edge and continue to grow along the rivet row through the region of residual compressive stress. For higher rivet squeeze forces, the region of beneficial residual stress through which the crack has to grow increases, increasing the overall fatigue life.

In addition to an increase in fatigue life, there is also an increase in fatigue scatter with squeeze force observed for the countersunk rivet specimens. This is indicated by the increase in the confidence intervals in Figure 6.8. This increase in scatter can be attributed to two 


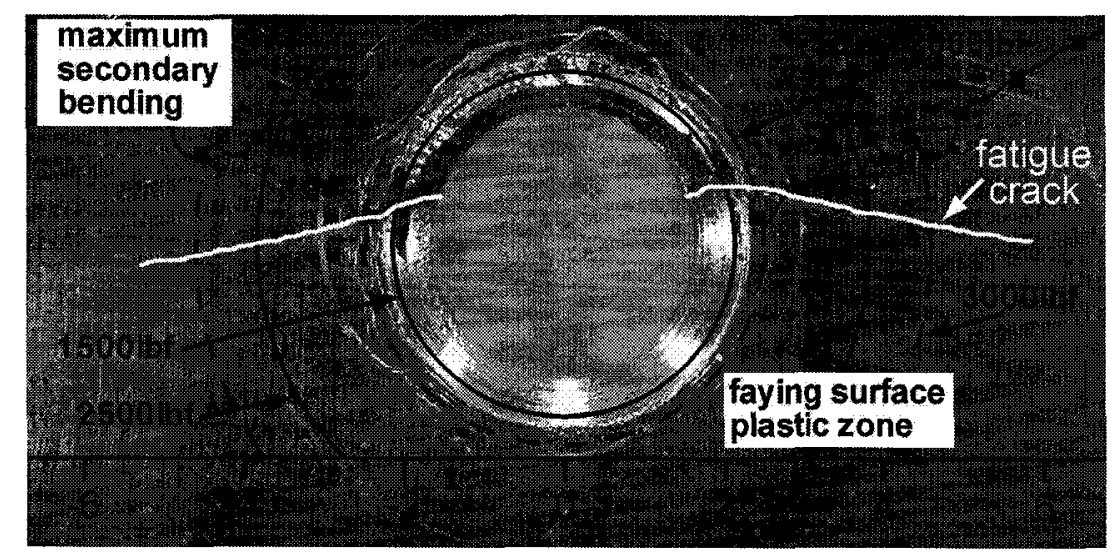

Figure 6.10: Typical location of fatigue damage observed in countersunk rivet joint fatigue specimens with FE outer sheet faying surface plastic zone radii

sources. First, at the higher squeeze forces, the wedge expansion mechanism is present. Since this mechanism is dependant on the initial protrusion of the rivet head above the sheet prior to installation, it introduces an additional source of scatter not present at the lower squeeze forces. The wedge expansion mechanism was discussed previously in Section 4.4.3. Secondly, at higher squeeze forces, crack nucleation shifts away from the hole edge and occurs as a result of fretting. This was observed during SEM reconstructions and will be discussed later in Section 6.5.2. As the processes involved in fretting induced crack nucleation are more heavily dependant on the local microstructure of the material, it also introduces an additional source of scatter.

Generally, the fatigue performance of the countersunk rivet specimens was lower than that of the equivalent universal rivet specimen. The criticality of the rivet hole as a fatigue notch for the countersunk rivet specimens, and the added stress concentration of the machined countersink contribute to this disparity. The lone exception observed in this study was at a squeeze force of $3000 \mathrm{lbf}$. At this squeeze force, the addition of the wedge expansion mechanism provides sufficient beneficial residual stresses in the countersunk rivet specimens to counterbalance the effect of the rivet hole and countersink. As the wedge expansion mechanism is not present in the universal rivet specimens, and because fatigue damage occurs away from the majority of beneficial residual stresses, the universal rivet specimens do not receive the same benefit. 


\subsection{Crack Growth Reconstruction Results}

Fractographic crack growth reconstructions were completed in order to quantify the effect of rivet installation on the behaviour of fatigue cracks. Based on the overall fatigue life results presented in the previous section and the required hours for each reconstruction, the scope of the crack growth reconstructions was reduced to two squeeze forces: $2000 \mathrm{lbf}$ and $3000 \mathrm{lbf}$. The results from this study are summarized in the following section. First, an overview of some general observations and obstacles encountered during the reconstructions is presented, followed by the countersunk and universal rivet joint results. A complete set of the crack reconstruction data can be found in Appendix F.

\subsubsection{General SEM Observations}

Several features commonly observed on fracture surfaces affected the reconstruction process. These features include the Alclad layer, fracture surface wear, and intersecting fracture planes, and are discussed in the following section.

The Alclad layer, although thin, is a prominent feature when viewing the fracture surface of 2024-T3 Alclad sheet under an SEM. The relative size of the Alclad layer relative to the 2024-T3 sheet is illustrated in Figure 6.11a. At the interface between the base 2024-T3 alloy and Alclad layer, a dramatic change in fracture surface topology occurs (Figure 6.11b). The lower yield strength of the Alclad layer results in ductile fracture which does not display the marker-band pattern as readily as the base alloy. As a result, identifying crack front locations within the Alclad layers was difficult. This was most problematic along the free sheet surface where the approaching crack front meets the Alclad layer at an acute angle as illustrated in Figure 6.11a. Extrapolating the crack front location through the thickness of the Alclad layer at such an acute angle resulted in inconsistent results for the free surface crack length. Consequently, efforts to quantify the free surface crack length had to be abandoned.

In order to minimize potential damage to the fracture surface during cyclic loading, a stress ratio $(R)$ equal to 0.1 was used to limit contact between the opposing fracture 


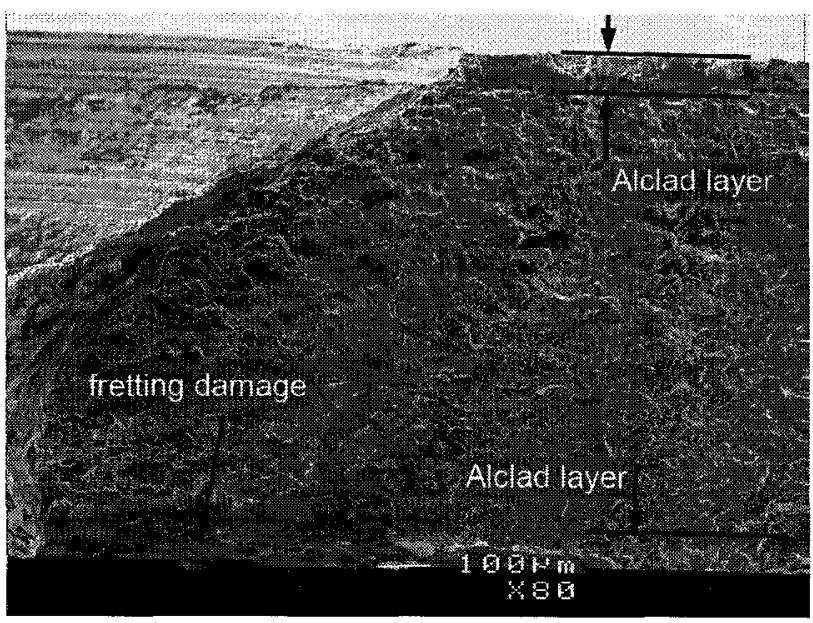

(a) relative size

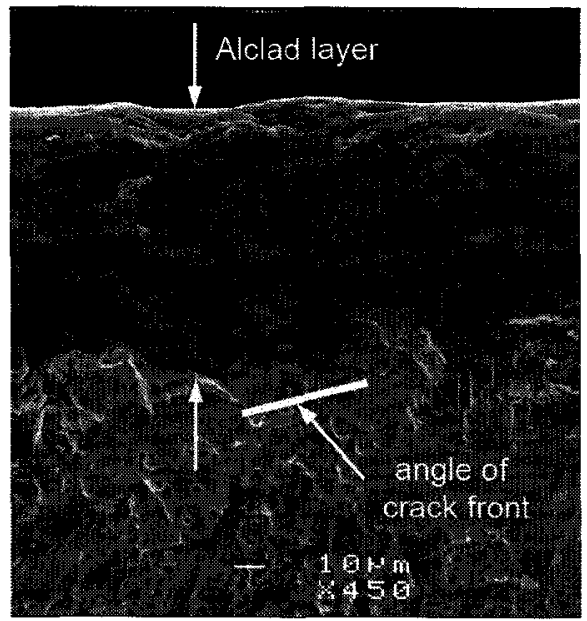

(b) fracture surface topography

Figure 6.11: Effects of Alclad layer on SEM reconstructions

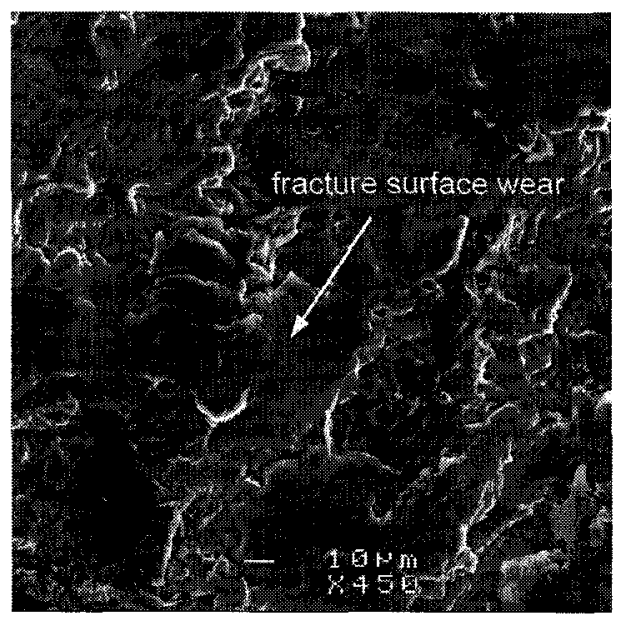

(a) fracture surface wear

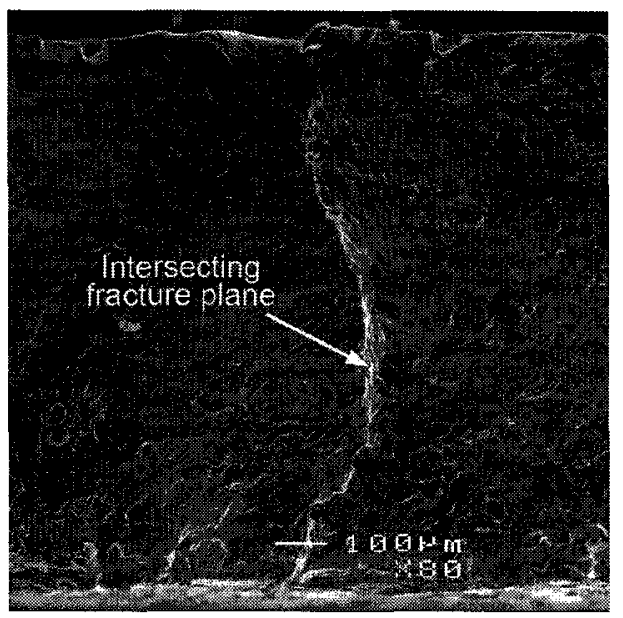

(b) intersecting crack planes

Figure 6.12: Fracture surface features which inhibited SEM reconstruction

surface faces. Despite this precaution, some instances of fracture surface damage as a result of scraping between opposing fracture surface faces were observed. An example of such damage is shown in Figure 6.12. In most cases, such wear damage occurred in isolated patches, and sufficient marker-bands could be identified around the damaged areas to complete a reconstruction.

Finally, for certain reconstructions, multiple crack nucleation events were found to occur which resulted in multiple crack planes. The observable fracture surface available for crack 
reconstruction consisted of overlapping portions of each of these crack planes. The intersections of these overlaps were easily identified by a step or ridge in the observable fracture surface as shown in Figure 6.12b. Since the reconstruction process relied on tracing the evolution of the crack front from its final position (the fatigue-static interface illustrated in Figure 6.5b), such discontinuities limited reconstruction efforts. Although marker-bands could be detected on the different fracture surfaces, the fatigue life associated with the crack fronts could not always be determined across the interface. This issue affected primarily the universal rivet joint reconstructions and is the reason why few universal rivet reconstructions were performed. Further details specific to the universal rivet joint reconstructions are given in Section 6.5.3.

\subsubsection{Countersunk Rivet Joint Reconstructions}

Typical crack front plots generated from countersunk rivet joint crack growth reconstructions for squeeze forces of 2000 and $3000 \mathrm{lbf}$ are given in Figures 6.13 and 6.14 respectively. These figures will be used to help illustrate the overall findings from the countersunk rivet joint reconstructions. The complete set of crack front plots and supporting reconstruction data are found in Appendix F.

\section{Crack Growth Behaviour}

The crack growth behaviour of all the countersunk rivet joint reconstructions is shown in Figure 6.15. As discussed in Section 6.5.1, due to the difficulties in identifying markerbands within the free surface Alclad layer, only results for the faying surface crack length are presented. Good repeatability was observed in the crack growth behaviour. Results for the crack position vs time cluster together well for the two squeeze forces as shown in Figure 6.15a. Agreement is improved when comparing crack growth rate vs crack length as shown in Figure 6.15b.

Over the range of crack lengths observed, the crack growth rate was approximately three times greater for the $2000 \mathrm{lbf}$ rivet squeeze force case compared to the $3000 \mathrm{lbf}$ case. This is easily visualized by comparing Figures 6.13 and 6.14 . The similar spacing of the displayed 


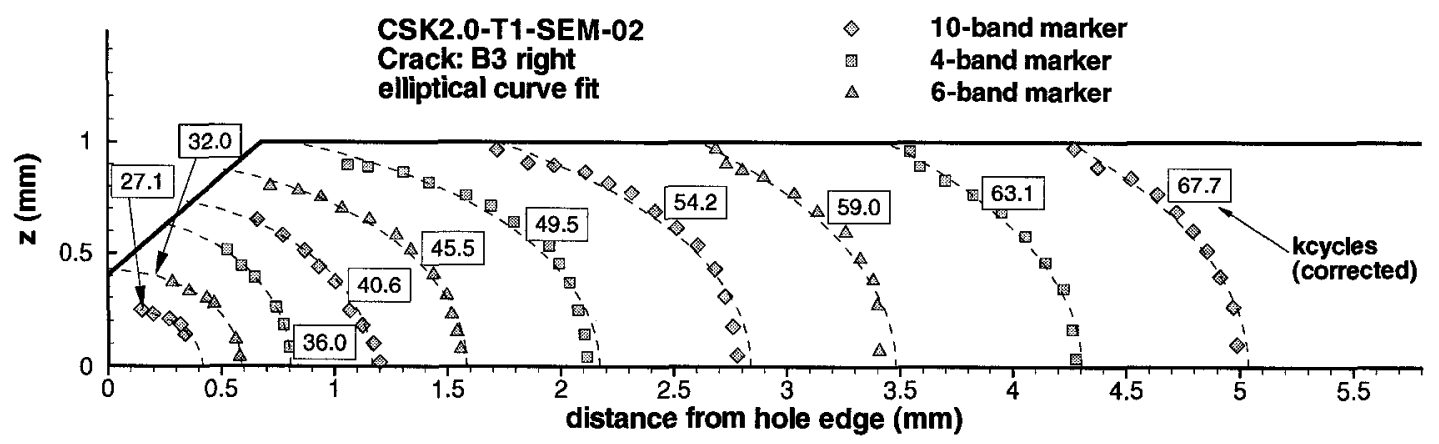

Figure 6.13: Typical crack front reconstruction for a countersunk rivet joint with $F_{S q}=$ $2000 \mathrm{lbf}$

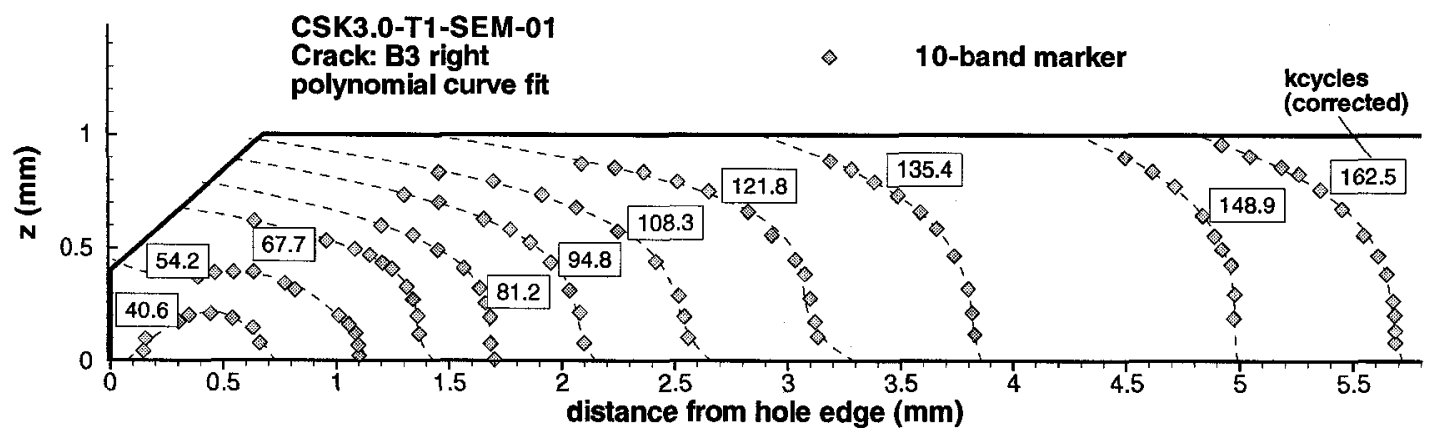

Figure 6.14: Typical crack front reconstruction for a countersunk rivet joint with $F_{S q}=$ $3000 \mathrm{lbf}$ (6- and 4-band markers removed for clarity)

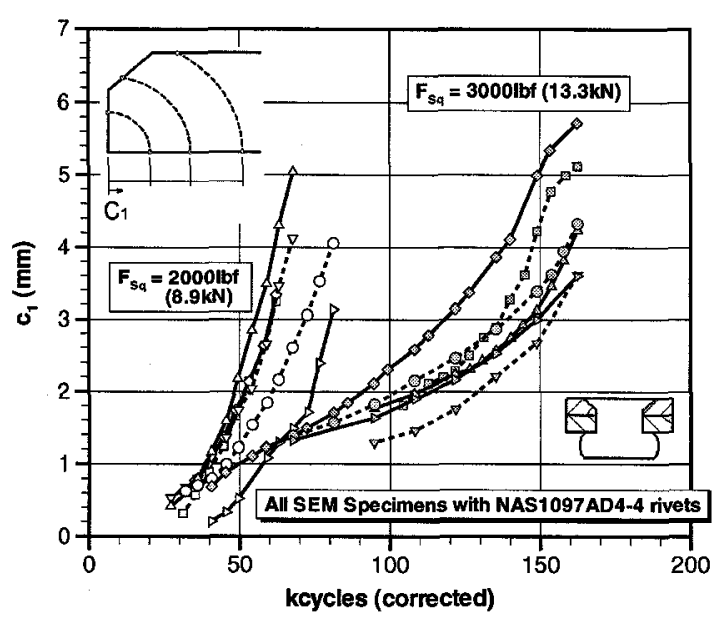

(a) growth curve

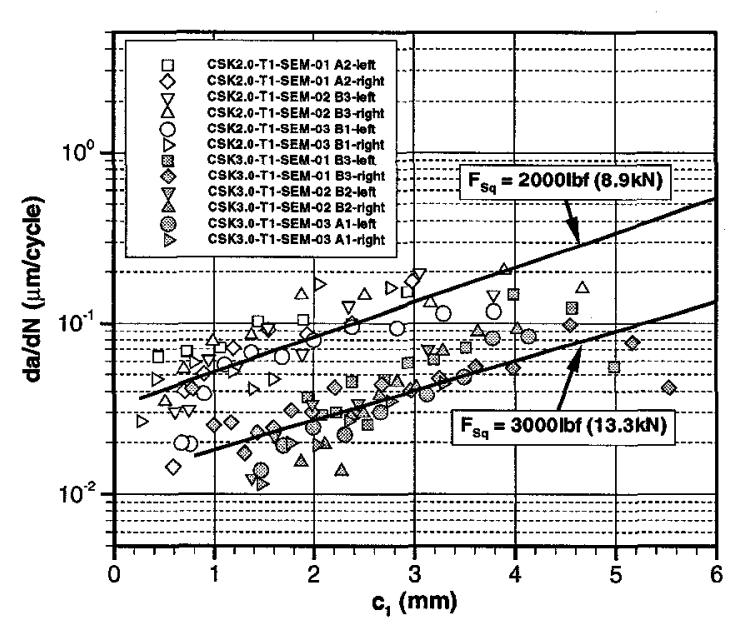

(b) growth rate

Figure 6.15: Crack growth behaviour for a countersunk rivet joint determined through SEM reconstructions 
crack fronts is an indication of the 3 -fold difference in crack growth rate as removal of the 6and 4-band markers in Figure 6.14 effectively triples the fatigue life between the displayed crack fronts compared to Figure 6.13.

Additional indications regarding the effect of the residual stress distribution on crack growth were also observed. For one of the $3000 \mathrm{lbf}$ reconstructions, there was an observed reduction in crack growth at approximately $c_{1}=5 \mathrm{~mm}$ (Figure 6.15a). This is believed to be related to the region of residual tensile tangential stress that is formed during rivet installation. The presence of the tensile residual stress would locally increase the crack growth rate within this region, resulting in the observed reduction in crack growth just beyond this region. From the finite element simulations performed in Chapter 4, the location of the peak residual tensile stress was found to to occur at approximately $c_{1}=5 \mathrm{~mm}$ for the 3000 lbf countersunk rivet installation case (see Figure 4.14b). This correlates with the observed reduction in crack growth at approximately $c_{1}=5 \mathrm{~mm}$. Unfortunately, none of the other $3000 \mathrm{lbf}$ reconstructions contained cracks of sufficient length to support this hypothesis. A similar effect should be observed for the 2000 lbf case, where the region of residual tangential stress peaks at approximately $c_{1}=2.5 \mathrm{~mm}$, however, this may be masked by the higher overall crack growth rates observed.

\section{Crack Nucleation Behaviour}

In addition to crack growth data, reconstruction of the crack front locations provides insight into when and where crack nucleation occurred. Results for the countersunk rivet joint reconstructions showed that there is a distinct shift in crack nucleation location for the two rivet squeeze forces analyzed. For a squeeze force of $2000 \mathrm{lbf}$, crack nucleation occurs at hole edge along the faying joint surface as can be inferred from location of the earliest crack front in Figure 6.13. For the higher squeeze force of $3000 \mathrm{lbf}$, nucleation shifts away from the hole edge, occurring approximately $0.4 \mathrm{~mm}$ away from the hole edge as shown in Figure 6.14 .

This shift in location is related to the dominant nucleation mechanism. At the lower squeeze force, crack nucleation is dominated by the presence of a notch (rivet hole) and its 
associated stress concentration. At the higher squeeze force, crack nucleation shifts to a region of heavy fretting damage. Within this region, cold-welding followed by disbonding of the asperities of the contacting joint surfaces occurs under cyclic loading, resulting in the formation of surface micro-cracks. Given sufficient bulk stress, these micro-cracks can propagate into the substrate, forming a fatigue crack.

Several factors contribute to the shift in crack nucleation location and mechanism. First, at the higher rivet squeeze force, larger residual compressive stresses are generated at the hole edge. These compressive stresses reduce the peak tensile stress at the hole edge, thus reducing its fatigue criticality. Second, the rivet clamping force increases with rivet squeeze force, exacerbating the fretting process. Although fretting and fretting fatigue are beyond the scope of the present investigation, a complementary investigation is being completed by Brown [63] on fretting fatigue in riveted lap joints. Preliminary results from Brown have confirmed the crack nucleation location observed at the higher squeeze force correlates with the edge-of-contact between the contacting sheets, where fretting damage and micro-crack densities are expected to be highest.

The crack reconstructions also provide some indication of when crack nucleation occurs. Although the exact time of nucleation cannot be established, crack front locations can be associated with specific times in the fatigue life, allowing the period over which nucleation occurred to be narrowed down. Results are shown in Table 6.2. The difficulty encountered with this method is that for the higher rivet squeeze force where fretting was the dominant nucleation mechanism, multiple cracks often formed, producing the ridge features described in Section 6.5.1. These ridge features often interrupted the reconstruction process, and although crack fronts near the nucleation location could be identified, a specific time within the fatigue life of the specimen could not be attributed to it. As a result, although the results in Table 6.2 seem to indicate that an increased squeeze force delays crack nucleation, this conclusion can not be made. What can be said, however, is that comparing the detection lives in Table 6.2 to the over average fatigue lives in Figure 6.8, crack nucleation was found to occur during the first $35 \%$ of the average overall fatigue life. 
Table 6.2: Nucleation period obtained from SEM reconstructions

\begin{tabular}{cccccc}
\hline Specimen & Crack & (kcycles) & Specimen & Crack & (kcycles) \\
\hline \multirow{2}{*}{ CSK2.0-T1-SEM-01 } & A1-left & 26.2 & CSK3.0-T1-SEM-01 & B3-left & 103.7 \\
& A1-right & 26.2 & & B3-right & 32.0 \\
\multirow{2}{*}{ CSK2.0-T1-SEM-02 } & B3-left & 41.6 & CSK3.0-T1-SEM-02 & B2-left & 81.2 \\
& B3-right & 41.6 & & B2-right & 81.2 \\
CSK2.0-T1-SEM-03 & B1-left & 32.0 & CSK3.0-T1-SEM-03 & A1-left & 54.2 \\
& B1-right & 40.6 & & A1-right & 67.7 \\
\hline average & & $\mathbf{3 4 . 7}$ & & & $\mathbf{7 0 . 0}$ \\
\hline
\end{tabular}

\subsubsection{Universal Rivet Joint Reconstructions}

The universal rivet joint reconstructions were less successful. The reconstruction process relied on being able to identify crack front locations and associate a fatigue life and crack length with these locations. Identifying the crack front locations was not problematic. Associating a fatigue life with each crack front location, however, was hampered by the presence of multiple fatigue cracks and fracture plane intersections. Defining a robust measure for crack length in the presence of multiple fatigue cracks was also difficult. For these reasons, only a limited number of reconstructions were performed for universal rivet joints.

The above described difficulties are illustrated in the reconstruction results shown in Figures 6.16 and 6.17 . Figure 6.16 best illustrates the difficulty of defining a robust measure for crack length. The main fracture surface was formed by two distinct fatigue cracks (centred at approximately $y=-1$ and $0.7 \mathrm{~mm}$ ) that eventually linked up to form a larger crack. During this link-up process, the shape and propagation rate of the crack front is dependant on the proximity and number of cracks involved. Simply tracking the location of the faying surface crack front location does not account for these factors, making it difficult to compare such results between different reconstructions. Reconstructions were further hampered by the presence of crack plane intersections. Such discontinuities in the fracture surface prevent the necessary accounting in determining the fatigue life associated with 


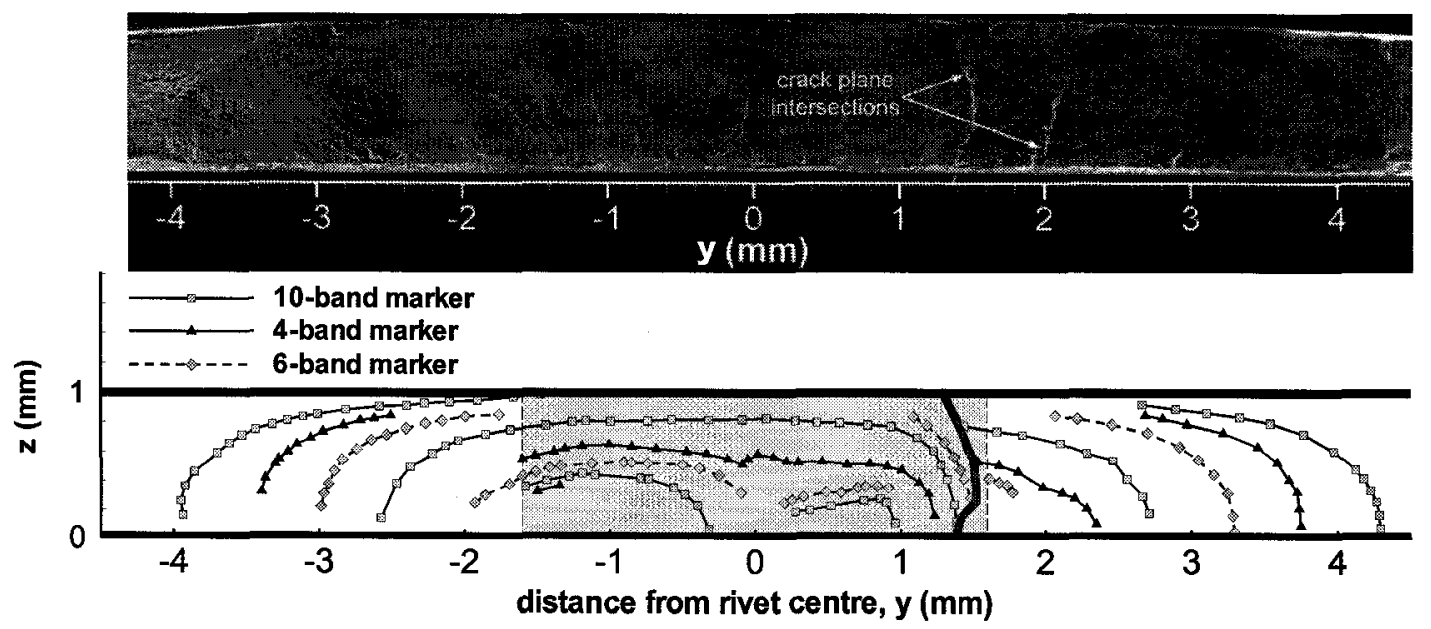

Figure 6.16: Crack front reconstruction for U2.0-T1-SEM-01 (arrows indicate intersecting fracture planes, shaded area indicates rivet hole location)

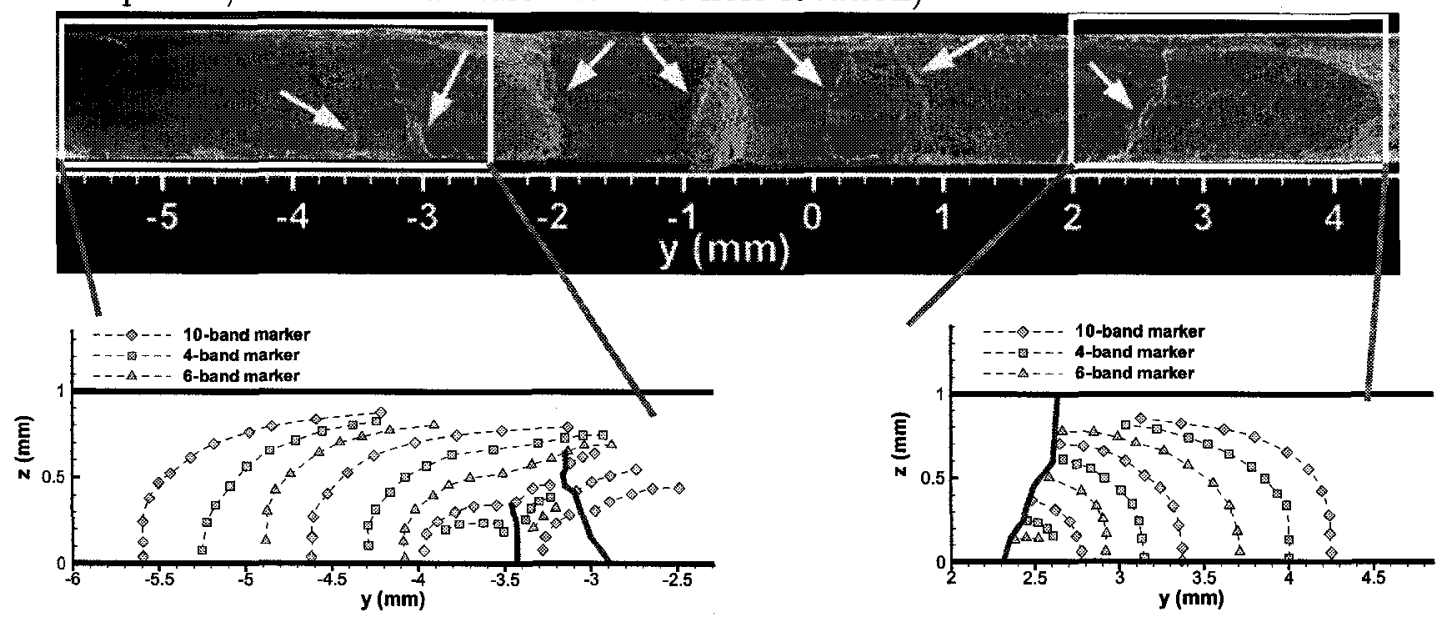

Figure 6.17: Crack front reconstruction for U3.0-T1-SEM-01 (arrows indicate intersecting fracture planes)

each identified crack front. As an example, in Figure 6.17, although crack fronts could be identified between $y=-3-2.5 \mathrm{~mm}$, associated fatigue lives could not be determined for the fronts due to the discontinuities in the fracture surface at $y=-3$ and $2.5 \mathrm{~mm}$.

Despite the limited quantitative data that could be obtained from the universal rivet joint reconstruction, some qualitative observations could be made. The presence of multiple fatigue cracks and fracture plane intersections indicate that fretting fatigue and the formation of multiple micro-cracks is a dominant crack nucleation mechanism for the universal joints. Although fretting was also observed as a nucleation mechanism for the countersunk 
rivet joints, the presence of the rivet hole along the fracture plane for these joints prevents a large number of micro-cracks from growing. Typically, a micro-crack would link-up with the rivet hole and become the dominant crack. For the universal rivet joints, the rivet hole does not lie along the fracture plane. As a result, multiple micro-cracks tend to grow and subsequently coalesce into larger fatigue crack.

\subsection{Conclusions}

Two series of fatigue tests have been carried out in order to quantify the effects of rivet installation on the fatigue performance of riveted lap joints. The first series aimed at quantifying the effects on overall fatigue life. Based on the results from this series, the following conclusions can be made:

- Considering failure in the outer sheet, a threshold squeeze force was observed for the universal rivet joint specimens below which the overall fatigue life was independent of squeeze force. Within this investigation, this threshold was found to be approximately $2500 \mathrm{lbf}$. At squeeze forces below this threshold, fatigue damage occurred outside of the region of compressive residual stress generated during riveting.

- Conversely, no such threshold was observed for the countersunk rivet joint specimens, where fatigue damage occurred near the joint net section and within the region of residual stress for all examined rivet squeeze forces.

- A minimum squeeze force was observed for the universal rivet joint specimens below which the critical failure location shifts from the outer to inner sheet location. This shift occurred at approximately $1500 \mathrm{lbf}$ within this investigation. No shift was observed for the countersunk rivet joint specimens within the range of examined squeeze forces. This is likely due to the added stress concentration provided by the machined countersink present only in the outer sheet.

- In general, the universal rivet joint specimens exhibited a longer overall fatigue life compared to the countersunk specimens with similar rivet squeeze forces. The lone 
exception was at the highest examined squeeze force of $3000 \mathrm{lbf}$ where the countersunk specimens performed better. An improved residual stress distribution due to the wedge expansion mechanism and the location of fatigue damage relative to the residual stress region result in this improvement for the countersunk rivet joint specimens.

The second series of fatigue tests were used to investigate the effects of rivet installation on crack nucleation and growth. Based on the results from this series, the following conclusions can be made:

- The SEM reconstructions performed on the universal rivet joint specimens were not successful because the presence of multiple fatigue cracks and fracture planes impeded the reconstruction process.

- For the countersunk rivet joint specimens, a 3-fold reduction in crack growth rate was observed when the rivet squeeze force was increased from 2000 to $3000 \mathrm{lbf}$.

- Crack nucleation was observed to occur within the first $35 \%$ of the average overall fatigue life. Although exact nucleation times could not be established, a time period within which nucleation occurred was established from the earliest detectable crack front.

Overall, the results of this investigation illustrate some key effects of rivet installation of the fatigue performance of riveted joints. First, the effect of rivet installation is also dependant on rivet type. Universal rivet joints were found to be less sensitive to variations in rivet squeeze force compared to countersunk rivet joints. Second, significant improvements in fatigue performance can be realized simply through increasing the rivet squeeze force. Although this is dependent on the first point, in the case of a countersunk rivet joint, the 3fold decrease in crack growth rate represents a significant improvement in damage tolerance of the joint. 


\section{Chapter 7}

\section{Discussion}

The present research describes the role of rivet installation on the fatigue performance of riveted lap joints using a combination of numerical and experimental methods. Greater understanding of this role would allow rivet installation to be exploited as a design variable, resulting in potential improvements to current and future airframe joint designs. To help realize such improvements, the role of rivet installation was examined for two airframe materials: monolithic 2024-T3 aluminum, commonly used in modern airframe structures, and GLARE laminates, a damage tolerant hybrid material that has recently been used in the Airbus A380 and has potential in future airframes.

The motivation for this research was first outlined in Chapter 1. Based on an established need for an improved understanding of rivet installation, the research objective, repeated below, was established:

To develop a better understanding of the role of rivet installation on the fatigue performance of riveted lap joints by studying, through numerical and experimental means, the effects of process variations on the formation of residual stresses, distribution of secondary bending stresses, and on crack growth.

This chapter provides a brief summary and discussion of the overall results from this research. Within the discussion, results will be related back to the above research objective. 


\subsection{Formation of Residual Stresses}

The formation of residual stresses due to riveting has traditionally been attributed to radial expansion of the rivet hole due to rivet deformation during riveting. Results from finite element simulations performed in this work (Chapters 3 and 4), however, revealed that the expansion levels resulting from riveting are too small to account for the entire residual stress field observed. Rather, through-thickness compression of the sheets between the rivet heads was found to be the major contributor to the formation of residual stress, particularly in fatigue critical outer sheet.

The consequences of this finding are significant. The development of a predictive analytical model for residual stress has been hampered by the non-linear radial expansion which occurs along the rivet shank and the complexity this adds to the problem. Throughthickness compression of the sheets during riveting is a simpler mechanism for which an analytical model could be developed. Additionally, this mechanism provides an opportunity to exploit the squeezing action of the rivet installation tooling. Prior to the rivet squeezing step, a modified rivet tool could be used to compress the sheets over a larger area and with a larger force to obtain an improved residual stress field. This pre-squeezing step could then reduce the sensitivity of the residual stress field to the rivet squeezing step.

In addition to the through-thickness compression of the sheets, a new mechanism which contributes to the formation of residual stress during countersunk rivet installation has been identified. Frictional slip was observed between the countersunk rivet head and contacting countersunk sheet during finite element simulations, resulting in further radial expansion of the countersunk sheet. This mechanism, which has been labelled wedge expansion, significantly increases the amount of radial expansion of the sheet due to deformation of the rivet shank alone, resulting in a larger region of compressive residual stress. The presence of wedge expansion was found to be dependant on the ratio of height-to-depth of the countersunk rivet head and machined countersink, as well as on the rivet squeeze force. The dependency on squeeze force may also be related to the relative size of the driven and countersunk rivet heads. 


\subsection{Secondary Bending}

Secondary bending produces tensile bending stresses along the faying joint surface, which promote fatigue crack nucleation. As a result, knowledge of the magnitude and location of peak secondary bending stresses is of importance to the designer. The finite element study described in Chapter 5 investigated the influence of rivet installation on the location and magnitude of these stresses. The relation was found to be geometric in nature. The location and magnitude of the secondary bending stresses within a particular sheet were dependant on the geometry of the adjacent rivet head. Factors influencing this geometry vary depending on the joint sheet in question. For the outer sheet, secondary bending is influenced by rivet type as it controls the geometry of the manufactured rivet head. Conversely, for the inner sheet, secondary bending is influenced by squeeze force (and rivet diameter) as they control the geometry of the driven rivet head.

Typically, fatigue failure is preferred in the outer sheet due to the accessibility of this area for inspections. Designers must then be aware of what factors influence the fatigue criticality of the outer sheet. Due to the role of secondary bending in crack nucleation, the importance of rivet selection during the design process is evident from the results of this work. Potentially, the secondary bending stress field within the outer sheet could be manipulated through changes to the geometry of the manufactured rivet head. Although current standardized rivet types exist, this dependency could be exploited in the design of a new fastener type.

Within Chapter 5, finite element results were also compared to the neutral line model; the current standard in secondary bending prediction capabilities. This simplified onedimensional model was found to be quite limited. The lack of discrete fastener locations and geometry within the model make it incapable of predicting variations in secondary bending along the joint width and around the fastener location. The basis for the neutral line model, however, is sound. The neutral line model calculates a bending moment reacted on by the rivet joint based on the joint eccentricity and loading. The over simplification comes from approximating a riveted lap joint with bonded joint with infinitely thin and 
rigid bond lines at the fastener row locations as the structure reacting the bending moment. Potentially, an improved model could be developed where the bending moment prediction from the neutral line model could be used as an input to a 2- or 3-dimensional model of a plate with a rigid pin. Given the importance of the geometry of the constraining rivet head identified within this research, the model could also incorporate a constraining head geometry on the end of the pin. Such a model would not only improve predictions of secondary bending stress, but would also allow the location of peak secondary bending stresses to be predicted and studied in more detail.

\subsection{Fatigue Performance}

Selection of rivet type and squeeze force was found to influence the local stress state in a riveted joint. The region of beneficial compressive residual stress is related to the rivet squeeze force and can reduce the rate of crack growth. This was observed in the SEM reconstructions described in Chapter 6 where a 3-fold reduction in crack growth rate was observed for a countersunk rivet joint when the rivet squeeze force was increased from 2000 to $3000 \mathrm{lbf}$. The location and magnitude of peak secondary bending stress is related to the geometry of the constraining rivet head, in addition to joint geometry and stiffness, and plays a key role in crack nucleation. This was also observed in Chapter 6 where fatigue cracks nucleated within the region of peak secondary bending stress identified in the finite element simulations described in Chapter 5. These effects, however, cannot be viewed independently.

A larger region of beneficial residual stress is of little use when it does not coincide with the location of crack nucleation and growth. This was observed in the relative insensitivity of the overall fatigue lives for universal rivet joint specimens to rivet squeeze force. Only at the highest squeeze forces examined did the beneficial residual stresses extend into the crack growth path. The opposite was true for the countersunk rivet joint specimens where cracks nucleated and grew through larger portions of the beneficial residual stress field. These results imply that rivet installation guidelines should be specific to rivet type. In the 
past, geometry based riveting guidelines were specified on the basis of rivet diameter. To fully exploit the fatigue life potential of a joint design however, different rivet squeeze forces may also need to be specified depending on what type of rivet is being used.

Fatigue test results from this research also highlighted the potential for exploiting rivet installation to improve fatigue performance. Although the rivet installation parameters varied within this research all fall within current acceptable riveting practice, up to 3-fold variations in overall fatigue performance were observed. By understanding the effects of these parameters and exploiting them during the design process, substantial improvements to fatigue performance can be realized.

\subsection{GLARE Laminates}

GLARE laminates are an attractive alternative to monolithic aluminum in airframes due to their superior damage tolerance resulting from the bridging effect of the fibre layer. This added damage tolerance is desirable, however, it does not negate the need for optimizing the design of structural details for fatigue performance. In this regard, the influence of rivet installation on the fatigue performance of GLARE was also considered in this research.

Although the presence of the fibre layer in GLARE increases damage tolerance, the fatigue mechanism in GLARE and in monolithic aluminum alloys is the same. Thus, for a riveted GLARE lap joint, crack nucleation will occur in regions of peak secondary bending and crack growth can be influenced by beneficial residual stress field. Differences between

the fatigue performance of GLARE and monolithic aluminum arise due to the influence of the fibre layer on the stress field within the aluminum layers. The question then arises, how does the fibre layer influence rivet installation and the resulting residual and secondary bending stress fields?

The elasticity of the fibre layer was found to influence the formation of residual stresses within the adjacent aluminum layers. In a manner similar to fibre bridging in the presence of a crack, the fibre layer bridges load around the portions of the aluminum layers that yield during rivet installation. This mechanism distributes the plastic strain incurred by the 
aluminum layers over a larger area, producing a larger region of beneficial residual stress. The elasticity of the fibre layer also increases the magnitude of the residual compressive stresses within the aluminum layers. Compressive hoop stresses are formed as a result of the elastic springback of the sheet which compresses the yield portion of the sheet. As the fibre layer does not yield, it stores larger amounts of elastic energy which increase this springback effect.

The lower stiffness of the fibre layer was found to influence secondary bending. This lower stiffness reduces the overall stiffness of the laminate, increasing the deflection resulting from secondary bending. These larger deflections, observed as larger rivet rotations, increase the secondary bending stress magnitudes near the rivet location. In practice, part of this increase can be negated through the use of thinner GLARE laminates. The improved damage tolerance of GLARE allows monolithic aluminum to be replaced with thinner GLARE laminates. This thickness reduction reduces joint eccentricity and the resulting secondary bending stresses.

Optimizing a lap joint design for GLARE requires taking these effects into account. Consequently, an optimum joint design for GLARE would have some key differences compared to its monolithic aluminum counterpart. First, the optimal rivet squeeze force would likely be lower for the GLARE joint. The presence of the fibre layer allows a similar residual stress field to be obtained in GLARE for a lower squeeze force. Furthermore, the potential for delamination formation at larger squeeze forces would play a role in the selection of squeeze force in a GLARE joint, while this is not a factor for an aluminum joint. Second, the rivet row spacing and possibly number of rivet rows in an optimal GLARE joint would be larger. The lower bending stiffness of GLARE exacerbates secondary bending in a GLARE lap joint. Increasing the number of rivet rows and/or the spacing between provides a way to reduce secondary bending and offset this penalty. Thirdly, the superior damage tolerance of GLARE resulting from the fibre bridging mechanism permits the reduction in rivet spacing. This spacing is limited by requirements for detecting fatigue damage within a given inspection interval. Wide spacings are necessary in monolithic aluminum due to the high crack growth rates of visible cracks. Reducing this spacing allows a larger number of 
rivets to be used, which in turn decreases the bearing stress at each rivet location. Lower bearing stresses would improve the crack nucleation life and potentially the overall fatigue life.

Indeed, an optimal GLARE lap joint design could vary significantly from its monolithic aluminum counterpart. The unique attributes of GLARE make it attractive as a damage tolerant alternative to monolithic aluminum and warrant consideration in the design process. 


\section{Chapter 8}

\section{Conclusions and Statement of Contribution}

The role of rivet installation on the fatigue performance of riveted lap joints was investigated. Specifically, the effects on the formation of residual stress, secondary bending, and crack nucleation and growth have been studied using a combination of numerical and experimental techniques.

Conclusions from this study must be viewed within the limitations of this work. The effects of rivet installation for all permutations and combinations of riveted joint design variables could not be investigated in this single study. Rather, a 2-row riveted lap joint configuration was chosen as a baseline configuration for the study. This baseline configuration consisted of two sheets joined by $3.2 \mathrm{~mm}$ rivets and with a rivet and row pitch of

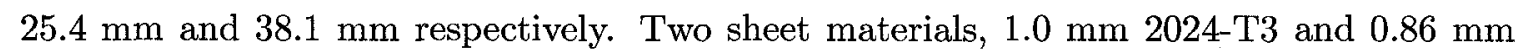
GLARE3-2/1-0.3, and two rivet types, NAS1097AD4-4 and MS20470AD4-4, were also investigated.

This chapter provides a summary of the overall set of conclusions reached during the course of the present investigation. In addition, the overall contribution provided by this research is stated. 


\subsection{Conclusions}

Conclusions drawn from this project are as follows:

\section{Riveting Induced Residual Stress (Chapters 3 and 4)}

- The region and magnitude of compressive residual stress increases with rivet squeeze force. Within the current study, no limiting or optimal squeeze force has been identified.

- The classical radial expansion mechanism to which residual stress formation is typically attributed to was observed. Expansion levels, however, were not sufficient to account for the observed residual stress field alone.

- Compression of the sheets between the two rivet heads during riveting was identified as an additional mechanism which contributes to the formation of residual stress. This mechanism played a larger role in the formation of residual stress than the radial expansion mechanism, particularly in the fatigue critical outer sheet where radial expansion levels were low.

- In countersunk joints, the residual stress field is sensitive to the depth of the countersink relative to the height of the countersunk rivet head. A small protrusion of the rivet head above the sheet surface prior to installation is necessary in order to ensure contact between the rivet head and the sheet during installation. This contact is important in the development of the through-thickness compression mechanism which dominates residual stress formation.

- The small protrusion of the rivet head above the joint surface prior to installation results in an additional mechanism for the formation of residual stress called wedge expansion. Wedge expansion is a result of frictional slip between the rivet head and countersunk sheet which causes the sheet to expand around the countersunk rivet head which acts as a wedge. This mechanism only occurs at high squeeze forces. 
Within the current study, and for a $0.07 \mathrm{~mm}$ initial protrusion of the rivet head, this threshold squeeze force was found to be $2500 \mathrm{lbf}$.

- The onset of the wedge expansion mechanism may be geometrical in nature. The threshold squeeze force observed in this study also corresponds to the point at which ratio of the diameters of the driven and manufactured rivet heads is 1 . This ratio was identified in studies completed by Müller as a threshold between the criticality of outer and inner sheet failure in experimental tests. The added residual stresses introduced by the wedge expansion mechanism in the outer sheet may contribute to this switch.

- Universal rivets do not provide any significant improvements in radial expansion within the outer sheet compared to countersunk rivets. For the same squeeze force, residual stress fields were similar for both rivet types except for instances where the wedge expansion mechanism was present. For such exceptions, a larger and more beneficial residual stress field was generated in the outer sheet for the countersunk rivet case.

\section{Secondary Bending (Chapter 5)}

- The neutral line model provides accurate predictions of secondary bending at a distance of three rivet diameters or greater from the rivet row centre line. Within three rivet diameters, significant variation in secondary bending was observed along the joint width, making the one-dimensional neutral line model inaccurate.

- The neutral line model underestimates the amount of secondary bending present near the rivet hole where crack nucleation occurs.

- The location and magnitude of peak secondary bending is affected by the geometry of the constraining rivet head. For bending within the outer sheet, this constraint is provided by the manufactured rivet head for which the geometry is dictated by rivet 
type. For bending in the inner sheet, this constraint is provided by the driven rivet head for which the geometry is influenced by the rivet squeeze force.

- Within the fatigue critical outer sheet, the location of peak secondary bending stresses is influenced by rivet type. For joints containing universal rivets, these stresses flow around the rivet hole while for joints containing countersunk rivets, these stresses intersect the rivet hole. Good correlation was found between the location of these peak secondary bending stresses and the location of crack nucleation, as shown in Figures 6.9 and 6.10. Differences in these crack nucleation locations would result in differing benefits due to the riveting induced residual stresses.

- The magnitude of secondary bending stresses near the rivet hole is larger for countersunk rivet joints in comparison to universal rivet joints. Remote from the rivet hole, the two rivet types produce similar results.

\section{GLARE Laminates (Chapters 3, 4, and 5)}

The fibre layer was found to influence both the residual stress and secondary bending stress fields in GLARE compared to monolithic 2024-T3 sheet. The following conclusions pertain to this influence.

- The elasticity of the fibre layer results in an apparent strain hardening effect in the laminate as a whole whereby load is bridged around yielded portions of the aluminum layer due to displacement compatibility of the aluminum and fibre layers. This effect causes plastic strain during riveting to be distributed over a larger area, producing a larger region of beneficial residual stress.

- Due to the lack of plasticity, the fibre layers in GLARE store more elastic strain energy during riveting than the aluminum layers. Upon completion of riveting, the laminate attempts to release this stored elastic strain energy during elastic springback. The driving force for springback is greater in the fibre layers and is resisted by the less resilient aluminum layers and rivet, resulting in larger residual compressive tangential 
stresses around the hole edge and larger residual rivet clamping forces through the joint thickness.

- The lower stiffness of the fibre layer reduces the overall bending stiffness of GLARE relative to monolithic 2024-T3 sheet. As a consequence of this higher flexibility, larger rivet rotations are observed due to secondary bending in GLARE joints. These larger rivet rotations result in larger secondary bending stresses which exacerbate crack nucleation.

- The anisotropy of the fibre layer in GLARE has a negligible effect on the secondary bending and residual stress fields compared to the effects described above.

\section{SEM Reconstructions (Chapter 6)}

- The SEM reconstructions performed on the universal rivet joint specimens were not successful because the presence of multiple fatigue cracks and fracture planes impeded the reconstruction process.

- For the countersunk rivet joint specimens, a 3-fold reduction in crack growth rate was observed when the rivet squeeze force was increased from 2000 to $3000 \mathrm{lbf}$.

- Crack nucleation was observed to occur within the first $35 \%$ of the average overall fatigue life. Although exact nucleation times could not be established, a time period within which nucleation occurred was established from the earliest detectable crack front.

\section{Overall Fatigue Life (Chapter 6)}

- Considering failure in the outer sheet, a threshold squeeze force was observed for the universal rivet joint specimens below which the overall fatigue life was independent of squeeze force. Within this investigation, this threshold was found to be approximately 2500 lbf. At squeeze forces below this threshold, fatigue damage occurred outside the region of compressive residual stress generated during riveting. 
- Conversely, no such threshold was observed for the countersunk rivet joint specimens, where fatigue damage occurred near the joint net section and within the region of residual stress for all examined rivet squeeze forces.

- A minimum squeeze force was observed for the universal rivet joint specimens below which the critical failure location shifts from the outer to inner sheet location. This shift occurred at approximately $1500 \mathrm{lbf}$ within this investigation. No shift was observed for the countersunk rivet joint specimens within the range of examined squeeze forces. This is likely due to the added stress concentration provided by the machined countersink present only in the outer sheet.

- In general, the universal rivet joint specimens exhibited a longer overall fatigue life compared to the countersunk specimens with similar rivet squeeze forces. The lone exception was at the highest examined squeeze force of $3000 \mathrm{lbf}$ where the countersunk specimens performed better. An improved residual stress distribution due to the wedge expansion mechanism and the location of fatigue damage relative to the residual stress region result in this improvement for the countersunk rivet joint specimens.

\subsection{Statement of Contribution}

This research, aimed at examining the influence of rivet type and installation on the fatigue performance of rivet joints, provides several contributions to the field of fatigue. First, a more comprehensive understanding of the formation of residual stresses during riveting was developed. Through-thickness stresses produced during riveting and the wedge-expansion mechanism were identified as dominant factors in the formation of residual stresses, revealing the limitations of simplified radial expansion analogies for riveting. Second, the localized influence of rivet type and installation on a loaded joint were investigated. Variations in secondary bending stress fields for universal and countersunk rivets were observed, helping explain differences in crack nucleation phenomena for these rivet types. Mapping of crack growth beneath the rivet head provided further understanding of the interaction 
of residual stresses, secondary bending, and tensile joint loading on the crack nucleation and early growth. Third, variations in the influence of riveting in 2024-T3 aluminum and GLARE3-2/1-0.3 joints were identified, leading the way for the design of GLARE-specific joints. Together, these contributions provide a greater understanding of the effects of the riveting process on fatigue necessary for further optimization of riveted joint designs. 


\section{List of References}

[1] R. P. G. Müller, An Experimental and Analytical Investigation on the Fatigue Behaviour of Fuselage Riveted Lap Joints: the Significance of the Rivet Squeeze Force and a Comparison of 2024-T3 and Glare 3. $\mathrm{PhD}$ dissertation, Delft University of Technology, 1995.

[2] R. de Rijck, Stress Analysis of Fatigue Cracks in Mechanically Fastened Joints. PhD dissertation, Delft University of Technology, 2005.

[3] H. Huth, "The influence of fastener flexibility on the prediction of load transfer and fatigue life for multi-row joints," ASTM Special Technical Publication, 1986.

[4] T. Swift, "Development of the fail-safe design features of the DC-10," ASTM Special Technical Publication 486, pp. 164-214, 1971.

[5] R. S. Piascik and S. A. Willard, "The characterization of wide spread fatigue damage in the fuselage riveted lap splice joint," NASA TP-97-206257, 1997.

[6] M. P. Szolwinski, The Mechanics and Tribology of Fretting Fatigue with Application to Riveted Lap Joints. PhD dissertation, Purdue University, 1998.

[7] M. P. Szolwinski and T. N. Farris, "Linking riveting process parameters to the fatigue performance of riveted aircraft structures," Journal of Aircraft, vol. 37, no. 1, pp. 130137,2000 .

[8] J. J. Homan, R. P. G. Müller, F. Pellenkoft, and J. J. M. Rijck, "Fatigue of riveted joints," in Fibre Metal Laminates an Introduction (A. Vlot and J. Gunnink, eds.), pp. 173-195, Dordrecht: Kluwer Academic Publishers, 2001.

[9] J. H. Park and S. N. Atluri, "Fatigue growth of multiple-cracks near a row of fastenerholes in a fuselage lap-joint," Computational Mechanics, vol. 13, no. 3, pp. 189-203, 1993.

[10] D. L. Ball, "Elastic-plastic stress analysis of cold expanded fastener holes," Fatigue and Fracture of Engineering Materials and Structures, vol. 18, no. 1, pp. 47-63, 1995. 
[11] J. B. Chang, "Prediction of fatigue crack growth at cold-worked fastener holes," Journal of Aircraft, vol. 14, no. 9, pp. 903-908, 1977.

[12] Y. C. Hsu and R. G. Forman, "Elastic-plastic analysis of an infinite sheet having a circular hole under pressure," Journal of Applied Mechanics, vol. 42, no. 2, pp. 347$352,1975$.

[13] X. Deng and J. W. Hutchinson, "The clamping stress in a cold-driven rivet," International Journal of Mechanical Science, vol. 40, no. 7, pp. 683-694, 1998.

[14] B. Langrand, E. Deletombe, E. Markiewicz, and P. Drazetic, "Riveted joint modeling for numerical analysis of airframe crashworthiness," Finite Elements in Analysis and Design, vol. 38, no. 1, pp. 21-44, 2001.

[15] G. Li and G. Shi, "Effect of the riveting process on the residual stress in fuselage lap joints," CASI, vol. 50, no. 2, pp. 91-105, 2004.

[16] G. Li, G. Shi, and N. C. Bellinger, "Study of the residual strain in lap joints," Journal of Aircraft, vol. 43, no. 4, pp. 1145-1151, 2006.

[17] L. Ryan and J. Monaghan, "Failure mechanism of riveted joint in fibre metal laminates," Journal of Materials Processing Technology, vol. 103, no. 1, pp. 36-43, 2000.

[18] M. R. Urban, "Analysis of the fatigue life of riveted sheet metal helicopter airframe joints," International Journal of Fatigue, vol. 25, pp. 1013-1026, 2003.

[19] R. C. Alderliesten, "Fatigue," in Fibre Metal Laminates an Introduction (A. Vlot and J. Gunnink, eds.), pp. 155-171, Dordrecht: Kluwer Academic Publishers, 2001.

[20] R. C. Alderliesten, M. Hagenbeek, J. J. Homan, P. A. Hooijmeijer, T. J. De Vries, and C. A. J. R. Vermeeren, "Fatigue and damage tolerance of Glare," Applied Composite Materials, vol. 10, pp. 223-242, 2003.

[21] G. H. J. J. Roebroeks, "Glare features," in Fibre Metal Laminates an Introduction (A. Vlot and J. Gunnink, eds.), pp. 23-28, Dordrecht: Kluwer Academic Publishers, 2001.

[22] T. W. de Jong, E. Kroon, and J. Sinke, "Formability," in Fibre Metal Laminates an Introduction (A. Vlot and J. W. Gunnink, eds.), pp. 337-353, Dordrecht: Kluwer Academic Publishers, 2001.

[23] T. W. de Jong, Forming of Laminates. PhD dissertation, Delft University of Technology, 2004.

[24] "FM94 Modified Epoxy Film," Tech. Rep. Technical Data Sheet 040102, Cytec Engineered Materials, May 1997. 
[25] T. J. de Vries, Blunt and Sharp Notch Behaviour of Glare Laminates. PhD dissertation, Delft University of Technology, 2001.

[26] C. A. J. R. Vermeeren, T. H. Beumler, J. L. C. G. De Kanter, O. C. Van Der Jagt, and B. C. L. Out, "Glare design aspects and philosophies," Applied Composite Materials, vol. 10, pp. 257-276, 2003.

[27] H. J. M. Woerden, J. Sinke, and P. A. Hooijmeijer, "Maintenance of Glare structures and Glare as riveted or bonded repair material," Applied Composite Materials, vol. 10, pp. 307-329, 2003.

[28] S. Krishnakumar, "Fibre metal laminates - the synthesis of metals and composites," Materials and Manufacturing Processes, vol. 9, no. 2, pp. 295-354, 1994.

[29] W. Z. Zhuang and G. R. Halford, "Investigation of residual stress relaxation under cyclic load," International Journal of Fatigue, vol. 23, pp. S31-S37, 2001.

[30] J. T. Maximov and A. P. Anchev, "Modelling of residual stress field in spherical mandrelling process," International Journal of Machine Tools \& Manufacture, vol. 43, pp. 1241-1251, 2003.

[31] J. T. Maximov, "Finite element analysis of the spherical mandrelling process of cylindrical holes," Finite Elements in Analysis and Design, vol. 40, pp. 1217-1232, 2004.

[32] A. Leon, "Benefits of split mandrel coldworking," International Journal of Fatigue, vol. 20, no. 1, pp. 1-8, 1998.

[33] A. T. Özdemir and R. Hermann, "Effect of expansion technique and plate thickness on near-hole residual stresses and fatigue life of cold expanded holes," Journal of Materials Science, vol. 34, pp. 1243-1252, 1999.

[34] M. J. Pavier, C. G. C. Poussard, and D. J. Smith, "A finite element simulation of the cold working process for fastener holes," Journal of Strain Analysis, vol. 32, no. 4, pp. 287-300, 1997.

[35] M. J. Pavier, C. G. C. Poussard, and D. J. Smith, "Finite element modelling of the interaction of residual stress with mechanical load for a crack emanating from a cold worked fastener hole," Journal of Strain Analysis, vol. 33, no. 4, pp. 275-289, 1998.

[36] M. J. Pavier, C. G. C. Poussard, and D. J. Smith, "Effect of residual stress around cold worked holes on fracture under superimposed mechanical load," Engineering Fracture Mechanics, vol. 63, pp. 751-773, 1999.

[37] P. F. P. de Matos, P. M. G. P. Moreira, P. P. Camanho, and P. M. S. T. de Castro, "Numerical simulation of cold working of rivet holes," Finite Elements in Analysis and Design, vol. 41, pp. 989-1007, 2005. 
[38] J. Kang, W. S. Johnson, and D. A. Clark, "Three-dimensional finite element analysis of the cold expansion of fastener holes in two aluminum alloys," Journal of Engineering Materials and Technology, vol. 124, no. 2, pp. 140-145, 2002.

[39] P. Papanikos and S. A. Meguid, "Three-dimensional finite element analysis of cold expansion of adjacent holes," International Journal of Mechanical Science, vol. 40, no. 10, pp. 1019-1028, 1998.

[40] T. N. Chakherlou and J. Vogwell, "The effect of cold expansion on improving the fatigue life of fastener holes," Engineering Failure Analysis, vol. 10, pp. 13-24, 2003.

[41] X. Zhang and Z. Wang, "Fatigue life improvement in fatigue-aged fastener holes using the cold expansion technique," International Journal of Fatigue, vol. 25, pp. 1249-1257, 2003.

[42] C. D. Rans, R. C. Alderliesten, and P. V. Straznicky, "Residual stresses in GLARE laminates due to the cold expansion process," in Proceedings of The Fifth CanadianInternational Composites Conference, CANCOM 2005, (Vancouver, Canada), Aug. 2005.

[43] D. L. Rich and L. F. Impellizzeri, "Fatigue analysis of cold-worked and interference fit fastener holes," Cyclic Stress-Strain and Plastic Deformation Aspects of Fatigue Crack Growth. ASTM STP No. 637, pp. 153-175, 1976.

[44] J. O. Hallquist, LS-DYNA Keyword User's Manual Version 970. Livermore, CA: Livermore Software Technology Corporation, 2003.

[45] DND, "Mil-hdbk-5h," 1998.

[46] M. Hagenbeek, "Thermal and mechanical properties of UD glass-fibre epoxy," Tech. Rep. B2V-04-01 (restricted), June 2004.

[47] E. M. A. H. van der Kuip, Fatigue Crack Initiation and Crack Growth in GLARE with Coldworked Holes. Masters thesis, Delft University of Technology, 2002.

[48] C. D. Rans, An Experimental Investigation into the Fatigue Behaviour of Dimple Countersunk GLARE Riveted Lap Joints. M.A.Sc. thesis, Carleton University, 2003.

[49] B. Langrand, E. Deletombe, E. Markiewicz, and P. Drazetic, "Numerical approach for assessment of dynamic strength for riveted joints," Aerospace Science and Technology, vol. 3, pp. 431-446, 1999.

[50] G. Li, G. Shi, and N. C. Bellinger, "Neutron diffraction measurement and FE simulation of residual strains and stress in fuselage lap joints," Tech. Rep. LTR-SMPL-2004-0003, Institute for Aerospace Research, National Research Council of Canada, January 2004. 
[51] S. A. Fawaz, Fatigue Crack Growth in Riveted Joints. PhD Dissertation, Delft University of Technology, 1997.

[52] A. Chu, A. Ranson, M. Sutton, and A. Peters, "Applications of digital image correlation techniques to experimental mechanics," Experimental Mechanics, vol. 25, no. 3, pp. 232-244, 1985.

[53] F. Hild and S. Roux, "Digital image correlation: from displacement measurement to identification of elastic properties - a review," Strain, vol. 42, pp. 69-80, May 2006.

[54] A. M. Brown, C. D. Rans, and P. V. Straznicky, "On achieving an optimal riveted lap joint design for fibre metal laminates," in Proceedings of The Sixth CanadianInternational Composites Conference, CANCOM 2007, (Winnipeg, Canada), Aug. 2007.

[55] P. F. P. de Matos, P. M. G. P. Moreira, I. Nedbal, and P. M. S. T. de Castro, "Reconstruction of fatigue crack growth in Al-alloy 2024-T3 open-hole specimens using microfractographic techniques," Engineering Fracture Mechanics, pp. 2232-2246, 2005.

[56] N. Grinberg, "Spacing of fatigue striations and crack growth rate," Soviet Materials Science, vol. 21, no. 2, pp. 153-160, 1985.

[57] I. Nedbal, J. Siegl, and J. Kunz, "Relation between striation spacing and fatigue crack growth rate in al alloy sheets," Advances in Fracture Research. Proceedings of the 7th International Conference on Fracture (ICF'7), pp. 3483-3491, 1989.

[58] J. Schijve, "The significance of fractography for investigations of fatigue crack growth under variable amplitude loading," Fatigue and Fracture of Engineering Materials and Structures, vol. 22, pp. 87-89, 1999.

[59] P. M. G. P. Moreira, P. F. P. de Matos, and P. M. S. T. de Castro, "Fatigue striation spacing and equivalent initial flaw size in al 2024-t3 riveted specimens," Theoretical and Applied Fracture Mechanics, vol. 43, pp. 89-99, 2005.

[60] R. S. Piascik and S. A. Willard, "The characterization of wide spread fatigue damage in the fuselage riveted lap splice," Tech. Rep. NASA-TP-97-206257, NASA, 1997.

[61] W. Elber, "The significance of fatigue closure," in Damage Tolerance in Aircraft Structures, no. ASTM STP 486, pp. 230 242, 1971.

[62] D. B. Schiff and R. B. d'Agostino, Practical Engineering Statistics. Wiley-Interscience, 1996.

[63] A. M. Brown and P. V. Straznicky, "Modelling fretting contact stresses in a single lap splice," in Proceedings of The Fith International Symposium on Fretting Fatigue, ISFF5, (Montreal, Canada), April, 2007. 


\section{List of Publications}

- C. D. Rans, R. C. Alderliesten, and P. V. Straznicky, "Effects of rivet installation on residual stress and secondary bending in a riveted lap joint," in Proceedings of The $48^{\text {th }}$ AIAA Structures, Structural Dynamics, and Materials Conference, (Honolulu, U.S.A.), April 2007.

- A. M. Brown, C. D. Rans, and P. V. Straznicky, "On achieving an optimal riveted lap joint design for fibre metal laminates," in Proceedings of The Sixth CanadianInternational Composites Conference, CANCOM 2007, (Winnipeg, Canada), Aug. 2007.

- C. D. Rans, R. C. Alderliesten, and P. V. Straznicky, "Riveting process induced residual stresses around solid rivets in mechanical joints," Journal of Aircraft, vol. 44, no. 1 , pp. 323-329, 2007.

- C. D. Rans, R. C. Alderliesten, and P. V. Straznicky, "Residual stresses in GLARE laminates due to the cold expansion process," in Proceedings of The Fith CanadianInternational Composites Conference, CANCOM 2005, (Vancouver, Canada), Aug. 2005.

- C. D. Rans, R. C. Alderliesten, and P. V. Straznicky, "Modelling of the rivet forming process in aluminum and GLARE for design against fatigue," in Proceedings of The $23^{\text {rd }}$ Symposium of the International Committee on Aeronautical Fatigue, ICAF 2005, (Hamburg, Germany), pp. 457-471, June 2005.

- C. D. Rans and P. V. Straznicky, "Avoiding knife-edge countersinks in GLARE through dimpling," Fatigue and Fracture of Engineering Materials and Structures, vol. 28 , no. 7 , pp. 633-640, 2005. 


\section{Appendix A}

\section{Finite Element Model Refinement}

This dissertation presents the results of several finite element studies as well as experimental validation of the results. The numerical simulation results were in all cases obtained from models that were refined to ensure convergence of the solution. For completeness, this appendix details the approach taken in refining each of the finite element models. The same approach is taken for each of the finite element models: cold-expansion (Chapter 3), rivet installation (Chapter 4), and splice loading (Chapter 5). As a result, the approach will be discussed in general terms with specific examples for each of the finite element models.

The performance of successive model refinements was measured on three levels: numerical convergence, stability and dynamic effects, and agreement with experimental results. Each level will be discussed in detail. All analyses were performed on an Intel dual Xeon EM64T with 8 gigabytes of memory. Early simulations were performed in the Microsoft Windows environment using the Windows version of LS-DYNA v.970. The majority of simulations, however, were performed in a unix environment with the corresponding unix version of LS-DYNA v.970.

\section{Numerical Convergence}

Numerical convergence, or simply convergence, refers to the process of refining a finite element mesh to ensure that the mesh is sufficient to capture the behaviour of interest. This process helps to limit and quantify the level of uncertainty in the model over which 
the user has direct control. Other sources of uncertainty within the model can be attributed to boundary conditions, material properties, and model geometry. These sources are more difficult to assess as they are subjective and open to interpretation. Boundary conditions are typically modelled as ideal simply-supported or fixed, while in reality there may be a degree of fixity along a boundary. Material properties and geometry can be quantified, but variabilities in manufacturing of real parts and materials can produce a level of uncertainty that is not under control of the analyst.

In performing a convergence study, it is critical to select an appropriate measure for assessing the level of convergence. It is conceivable that results for one portion of the model may have converged while in other portions they have not. For this reason, the convergence level for each of the finite element models in this work was assessed based on the primary stress result of interest. For the three finite element models, these correspond to:

- Cold Expansion - Mid-plane residual tangential stress distribution

- Rivet Installation - Faying surface residual tangential stress distribution (both inner and outer sheet)

- Splice Loading - Peak secondary bending stress distribution along fastener row

In some of the models, variations in load (rivet squeeze force) and geometry (rivet type and material) needed to be accommodated. Convergence checks were performed on the effects of these variations; however, the majority of convergence efforts were focused on a rivet squeeze force value of $3000 \mathrm{lbf}$. As both rivet and sheet type directly affected the mesh, these variables were studied in detail as well.

Successive mesh refinement steps did not employ a uniform refinement of the mesh. Mesh refinement was biased towards the fastener hole region as it was the primary region of interest in each of the analyses. Table A.1 provides details on the mesh refinement level in each of the finite element models used in this work. The level of refinement is described by the number of unconstrained degrees of freedom (DoF) present in the mesh. 
Table A.1: Mesh refinement level details used in finite element convergence studies

\begin{tabular}{|c|c|c|c|c|}
\hline FE Model & Sheet Material & Rivet Type & Mesh & DoF \\
\hline \multirow{3}{*}{ Cold Expansion } & \multirow{3}{*}{ 2024-T3 } & \multirow{3}{*}{ N/A } & coarse & 36,000 \\
\hline & & & intermediate & 65,000 \\
\hline & & & fine & 109,000 \\
\hline \multirow{2}{*}{ Cold Expansion } & \multirow{2}{*}{ GLARE3-2/1-0.3 } & \multirow{2}{*}{$\mathrm{N} / \mathrm{A}$} & coarse & 98,000 \\
\hline & & & fine & 135,000 \\
\hline \multirow{4}{*}{ Rivet Installation } & \multirow{4}{*}{ 2024-Т3 } & countersunk & coarse & 220,000 \\
\hline & & countersunk & fine & 304,000 \\
\hline & & universal & coarse & 293,000 \\
\hline & & universal & fine & 368,000 \\
\hline \multirow{4}{*}{ Rivet Installation } & \multirow{4}{*}{ GLARE3-2/1-0.3 } & countersunk & coarse & 298,000 \\
\hline & & countersunk & fine & 339,000 \\
\hline & & universal & coarse & 357,000 \\
\hline & & universal & fine & 405,000 \\
\hline \multirow{5}{*}{ Splice Loading } & \multirow{5}{*}{ 2024-T3 } & countersunk & coarse & 145,000 \\
\hline & & countersunk & intermediate & 667,000 \\
\hline & & countersunk & fine & $1,208,000$ \\
\hline & & universal & intermediate & 889,000 \\
\hline & & universal & fine & $1,448,000$ \\
\hline \multirow{4}{*}{ Splice Loading } & \multirow{4}{*}{ GLARE3-2/1-0.3 } & countersunk & intermediate & $1,013,000$ \\
\hline & & countersunk & fine & $1,329,000$ \\
\hline & & universal & intermediate & $1,288,000$ \\
\hline & & universal & fine & $1,620,000$ \\
\hline
\end{tabular}


The level of convergence between successive refinements was measured based on the percent change in the result of interest at any point between the two refinements. A baseline convergence level of $5 \%$ was sought after in all of the convergence studies. This was achieved for all of the models with one exception. Regions of contact where large contact forces were present could become unstable, exhibiting nodal penetrations through the contact interface, as further mesh refinements were applied. This was primarily a problem at the intersection of the faying sheet surfaces and fastener hole, where contact between the two sheets and rivet/mandrel occurs. As a result, along the faying sheet surfaces, the $5 \%$ convergence target was not always met within $0.5 \mathrm{~mm}$ of the hole edge $(r / R=1.3)$. Within this region, the convergence target was relaxed to $10 \%$, which was never exceeded. A sample set of the convergence study comparisons are given in Figure A.1 for the cold expansion analysis, Figure A.2 for the rivet installation analysis and Figure A.3 for the splice loading analysis.

Although the convergence criteria is met for the results shown in Figure A.3, there is a clear discontinuity in the results seen at approximately $Y=0.1$ in. The behaviour occurs due to a difference in location of the resultant data between the different meshes. The secondary bending factor was calculated at nodal locations as the faying and free surface meshes are the same. Stress results for these surfaces are mapped to their undeformed nodal mesh. As

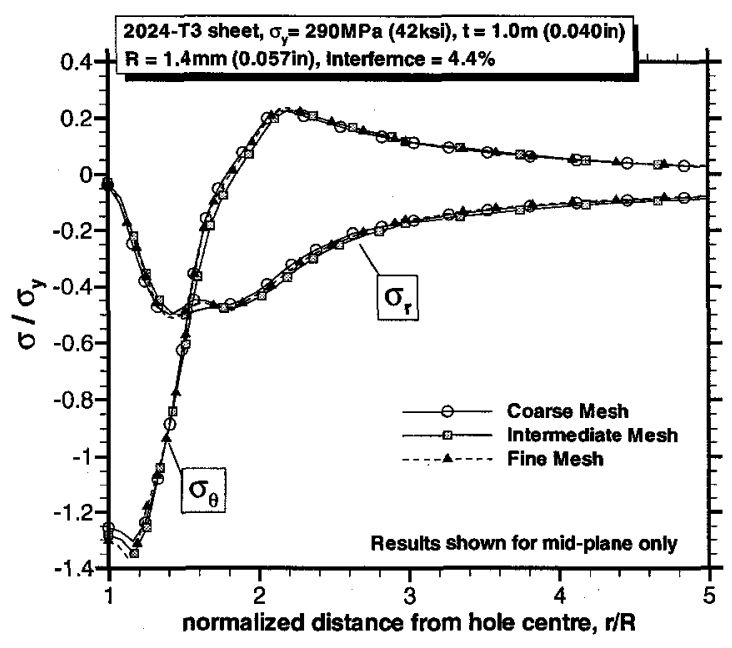

Figure A.1: Sample mesh convergence study results for cold expansion FE analysis 


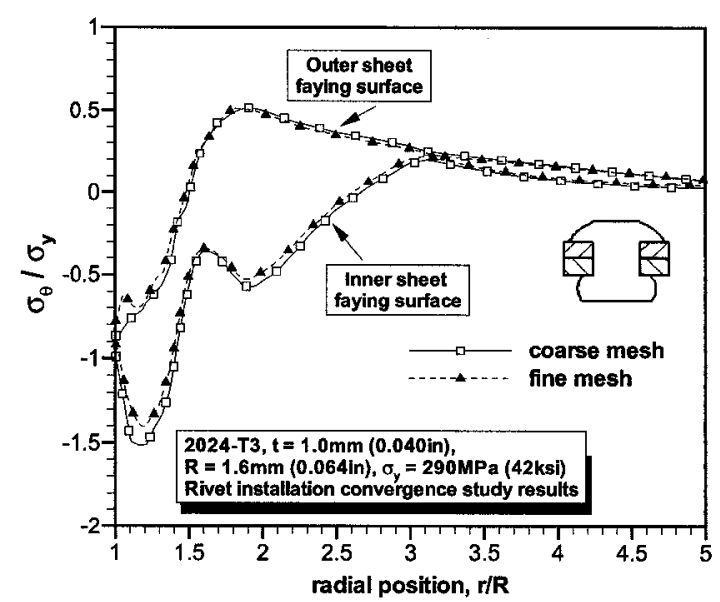

(a) universal rivet

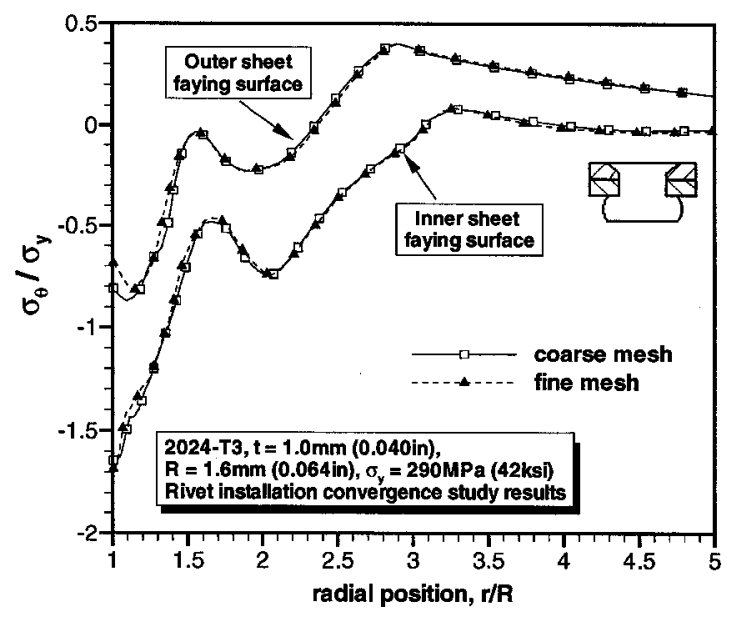

(b) countersunk rivet

Figure A.2: Sample mesh convergence study results for rivet installation FE analysis

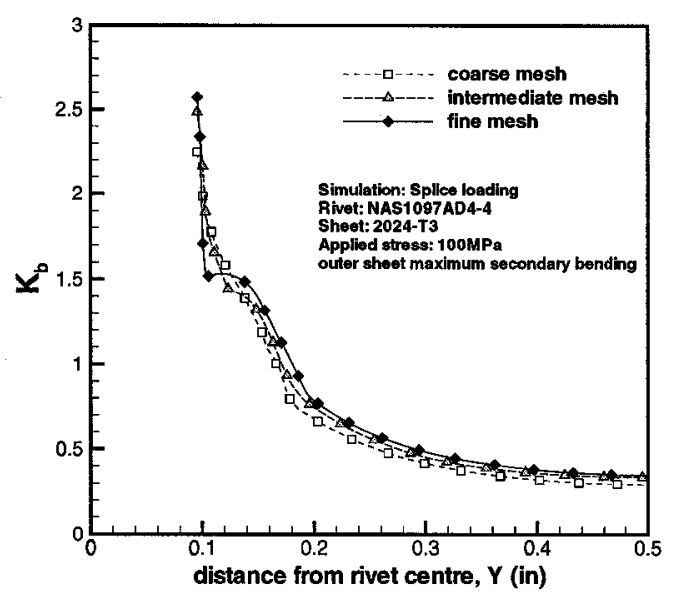

Figure A.3: Sample mesh convergence study results for splice loading FE analysis

these are the same for both surfaces, the results for the two surfaces are merged onto one surface on which the secondary bending factor is calculated. The location and magnitude of maximum bending is determined by dividing this surface into narrow vertical columns and searching for a nodal location in this column which contains the largest secondary bending factor. Thus, small discontinuities in the position of maximum bending are possible using this method as it uses only nodal results only rather than interpolating between nodes. 


\section{Stability and Dynamic Effects}

Performing a mesh convergence only ensures numerical convergence of the results for the simulation performed. Another important step in the verification of any finite element analysis is to check the major assumptions and simplifications made in the analysis in order to quantify their effects. Such assumptions and simplifications can include choice of material models, boundary conditions, and other simulation parameters that may deviate from reality in order to simplify the modelling process.

The primary assumption made in the set of finite element analyses performed in this dissertation was that the processes simulated (cold expansion, rivet installation, and splice loading) are all quasi-static. This assumption asserts that dynamic effects within the process are negligible. With such an assumption, it is possible to employ techniques to reduce computational times.

With the explicit LS-DYNA solver used in this work, the computational time required for an analysis is directly proportional to the simulated time divided by the integration time step. The integration time step is determined automatically based on the time required for a sound wave to travel the length of the smallest element in the mesh [44]. As the mesh size is dictated by numerical convergence requirements, two options are presented to the analyst for reducing computational times:

- Increase the mass density within the material models, thus reducing the speed of sound through the finite element mesh. This effectively increases the time step size automatically determined by the solver. This process is known as mass scaling.

- Decrease the simulated time over which the process occurs. This process is known as time scaling.

The second technique, time scaling, was employed in this work. Rather than simulating the duration of rivet installation, splice loading, and cold expansion on the order of seconds, as they occur in practice, the simulation times were reduced to fractions of a second. If the assumption of a quasi-static process holds, the increase in the rate at which the process 
occurs should not influence the simulation and dynamic effects (indicated by kinetic energy in the system) should remain negligible.

Another simplification that was made within the finite element analyses was the application of hourglass control. The issue of hourglass deformation modes is discussed in detail in Section 3.3.1. To prevent these unstable deformation modes, hourglass control applies artificial damping around an element as it passes through one of these modes. The application of damping consumes energy from the system, which can influence the results of the simulation. To ensure this does not occur, the analyst must monitor the amount of damping energy associated with hourglass control (hourglass energy) and ensure that it also remains negligible with respect to the total energy of the system.

The approach taken to assess the effects of time scaling and hourglass control on the finite element results was two fold. First, kinetic and hourglass energy levels over the duration of the finite element analyses were monitored relative to the total energy of the system. Although it is difficult to define a limit at which kinetic and hourglass energies can be considered negligible, this approach provided a quick method for assessing if this assumptions is violated. Second, the simulated time $\left(T_{\text {simulation }}\right)$ for each analysis was varied to see if this parameter had an influence on the stress results. If the assumption that kinetic or hourglass energy remain negligible had been violated in any of the simulations, changes to the simulated time would influence results of the analysis.

Typical energy plots used to assess whether kinetic and hourglass energies remained negligible throughout the analyses are shown in Figure A.4. Generally, kinetic and hourglass energies remain below $2 \%$ of the total energy in the system, suggesting that they are indeed negligible. At the begining of each simulation, the kinetic energy percentage of the overall system energy attributed to kinetic energy appear to be large, greatly exceeding the $2 \%$ value. This is a result of initial gaps between the riveting tools and rivet or mandrel and sheet in the simulations. Until contact between these contact pairs is established, the motion of these parts accounts for a large percentage of the total energy. During this time, however, the total energy itself is very small, making the percentage of kinetic energy sensitive to 

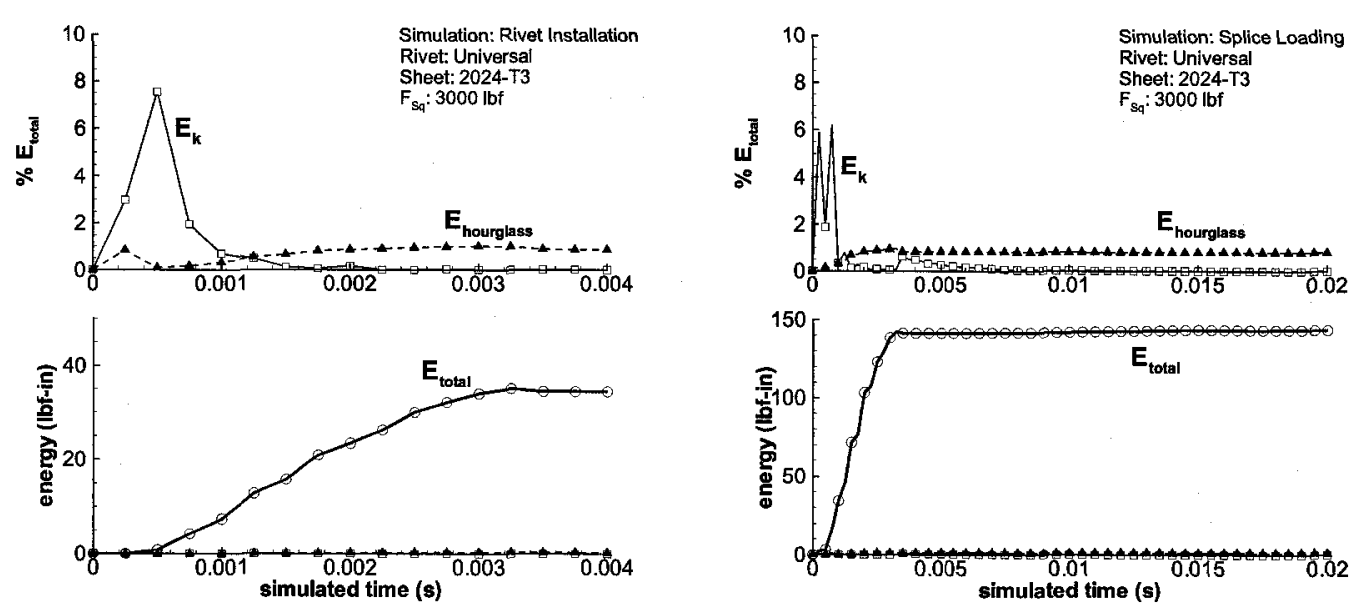

(a) rivet installation analysis

(b) splice loading analysis
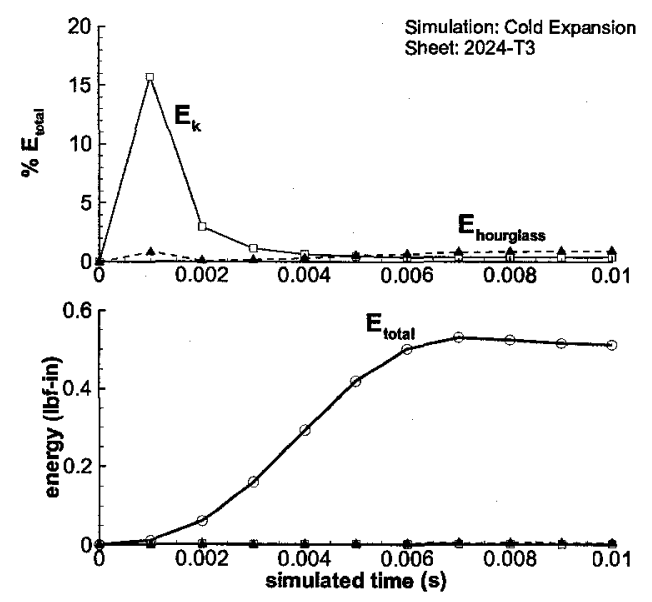

(c) cold expansion analysis

Figure A.4: Sample energy plots used to monitor kinetic and hourglass energy levels

round off error in the computed energies (see initial oscillation of kinetic energy in Figure A.4b). Thus, as the total energy itself is negligible at this stage, the kinetic energy is also negligible.

A rise in kinetic energy is shown in Figure A.4b at approximately $t=0.005 \mathrm{sec}$ for the splice loading simulation. This rise in kinetic energy corresponds to the time at which the tension load is applied to the joint. This rise in kinetic energy occurs due to rotation of the joint under the applied load and not due to instabilities in the model. 


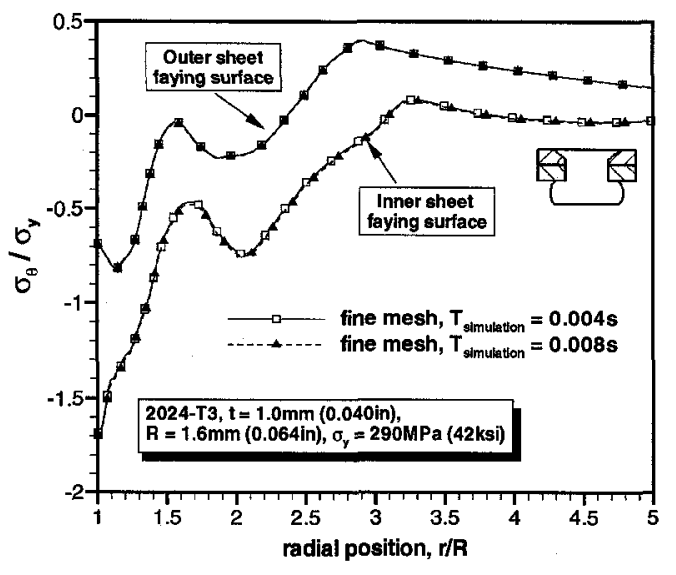

(a) rivet installation analysis

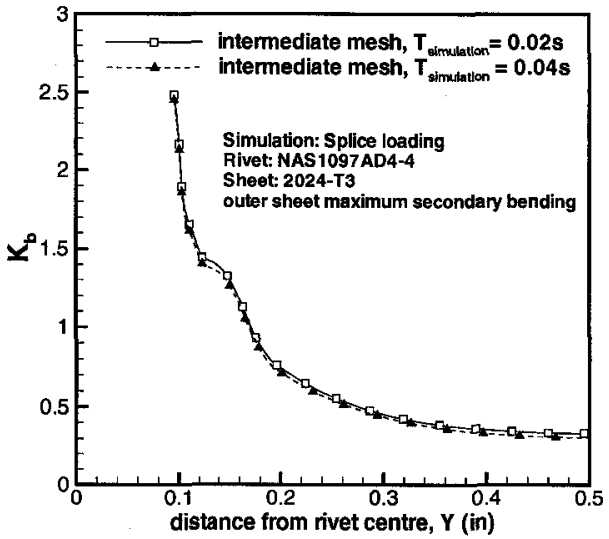

(b) splice loading analysis

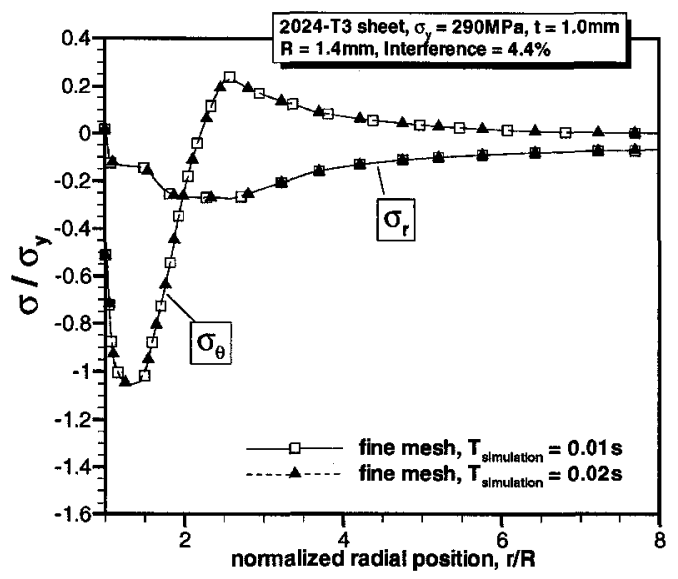

(c) cold expansion analysis

Figure A.5: Sample time scaling sensitivity results

Typical results from simulations studying the effects of simulation time on analyses results are shown in Figure A.5. Doubling the simulation time for each of the analyses did not result in appreciable changes to the analysis results. As with the mesh convergence study, results were checked to see if variations larger than $5 \%$ existed. Such large variations did not appear in any of the cases.

From the results of the two above assessments, it was deemed that time scaling and hourglass control did not influence the results of each of the analyses. 


\section{Experimental Validation}

As a final step in establishing confidence in the results of the finite element analysis presented in this dissertation, analysis results were compared directly to experimental or analytical results of the modelled process/event. The nature and setup of the experiments used in this validation varied for each of the analyses and are described within the dissertation and will not be repeated here. These results can be found in Sections 3.4.1, 4.3.5, and 5.3.2 for the cold expansion, rivet installation, and splice loading analyses respectively.

\section{Summary of Finite Element Models}

A summary of the finite element mesh sizes and simulation times chosen for each of the finite element analyses based upon the above described refinement process is given in Table A.2. Typical computation times are also reported for the analyses. For the rivet installation and splice loading analyses, these computation times are based on the $F_{S q}=3000 \mathrm{lbf}$ case.

Table A.2: Resultant FE mesh size and computation times

\begin{tabular}{|c|c|c|c|c|c|}
\hline FE Model & Sheet Material & Rivet Type & DoF & $\begin{array}{c}T_{\text {simulation }} \\
\text { (s) }\end{array}$ & $\begin{array}{c}\text { Processing Time } \\
\text { (s) }\end{array}$ \\
\hline \multirow{2}{*}{ Cold Expansion } & 2024-T3 & $\mathrm{N} / \mathrm{A}$ & 109,000 & 0.01 & 17297 \\
\hline & GLARE3-2/1-0.3 & $\mathrm{N} / \mathrm{A}$ & 135,000 & 0.01 & 24946 \\
\hline \multirow{2}{*}{ Rivet Installation } & \multirow{2}{*}{ 2024-T3 } & countersunk & 304,000 & 0.004 & 33459 \\
\hline & & universal & 368,000 & 0.004 & 42436 \\
\hline \multirow{2}{*}{ Rivet Installation } & \multirow{2}{*}{ GLARE3-2/1-0.3 } & countersunk & 339,000 & 0.004 & 37145 \\
\hline & & universal & 405,000 & 0.004 & 43099 \\
\hline \multirow{2}{*}{ Splice Loading } & \multirow{2}{*}{ 2024-T3 } & countersunk & $1,208,000$ & 0.02 & 230161 \\
\hline & & universal & $1,448,000$ & 0.02 & 264338 \\
\hline \multirow{2}{*}{ Splice Loading } & \multirow{2}{*}{ GLARE3-2/1-0.3 } & countersunk & $1,329,000$ & 0.02 & 299190 \\
\hline & & universal & $1,620,000$ & 0.02 & 350566 \\
\hline
\end{tabular}




\section{Appendix B}

\section{Neutron Diffraction Verification of Rivet Installation FE Model}

Several indirect verification studies were completed in order to assess the performance of the rivet installation finite element model detailed in Chapter 4. One of these studies involved comparing residual strain results from the finite element model to a neutron diffraction study completed by Li et al. $[16,50]$. The required changes to the FE model that were made to facilitate the comparison and the full set of results used in the comparison are presented here. Further details on the neutron diffraction technique and the test setup used by $\mathrm{Li}$ et al. can be found in their publications $[16,50]$.

\section{Required FE Model Changes}

Changes to the FE model geometry were needed in order to replicate the conditions of the neutron diffraction study. Changes included increasing the sheet thickness from $1.0 \mathrm{~mm}$ to $2.0 \mathrm{~mm}$ and increasing the rivet diameter and changing the countersink style from NAS1097 to MS20426. Additional geometry changes are summarized in Figure B.1. Both the $44.5 \mathrm{kN}$ and $53.4 \mathrm{kN}$ squeeze forces considered in the neutron diffraction study were simulated with the modified FE model. 


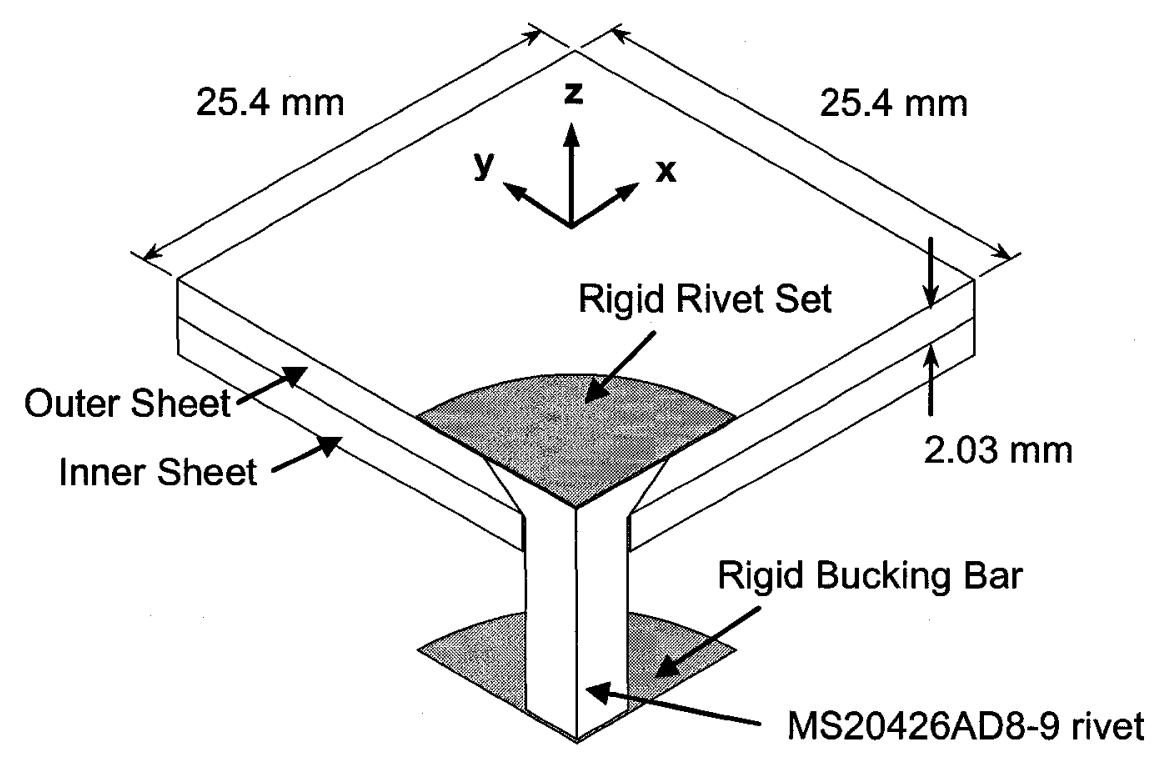

Figure B.1: Schematic of modified rivet installation finite element model

\section{Comparison Results}

Comparison of the finite element and neutron diffraction residual strains are presented in Figures B.2 to B.7. In addition to the neutron diffraction data, results from a 2-dimensional axisymmetric finite element model developed by Li et al. are also included for comparison $[16,50]$. The level of agreement between the FE and neutron diffraction results varied depending on the residual strain component, the sheet being looked at, and the squeeze force. Generally, better agreement was found for the lower squeeze force compared to the higher squeeze force. Additionally, tangential and clamping strain components showed better agreement than the radial strain component. There are several potential causes for the observed discrepancies:

1. Results from the neutron diffraction study are averaged over a given volume while the FE results are resolved for discrete points. The gauge volume for the neutron diffraction study was $1 \mathrm{~mm}^{3}$, although the shape of the volume varied for different strain components and locations. 
2. Positional tolerances on the gauge locations within the neutron diffraction study and FE model.

3. Variations in local material properties due to material grain size and orientation.

Results between the current finite element model and the 2D model developed by $\mathrm{Li}$ et $a l$. agree extremely well. The most significant discrepancies between the two models occurs for the residual clamping strain. This is likely caused by differences in the rivet material models used in both studies, which would influence the residual clamping force the rivet imparts to the surrounding sheet. 


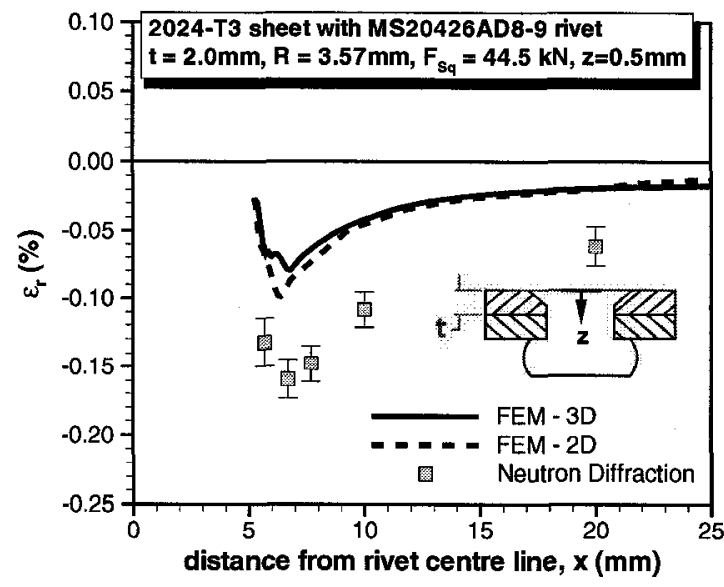

(a) $F_{S q}=44.5 \mathrm{kN}$

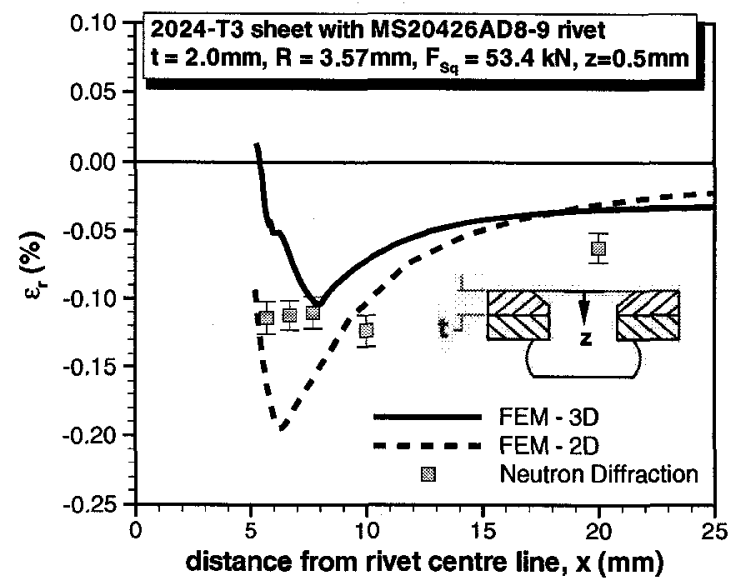

(b) $F_{S q}=53.4 \mathrm{kN}$

Figure B.2: Comparison of outer sheet residual radial strains

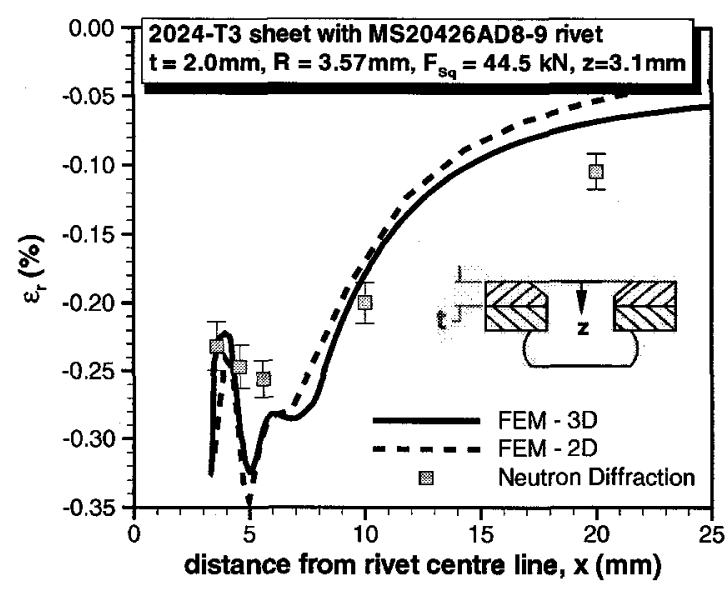

(a) $F_{S q}=44.5 \mathrm{kN}$

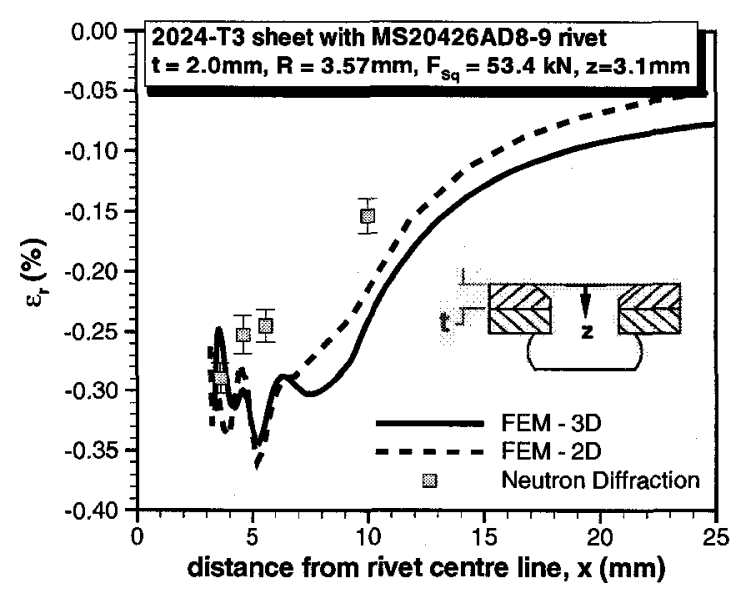

(b) $F_{S q}=53.4 \mathrm{kN}$

Figure B.3: Comparison of inner sheet residual radial strains 


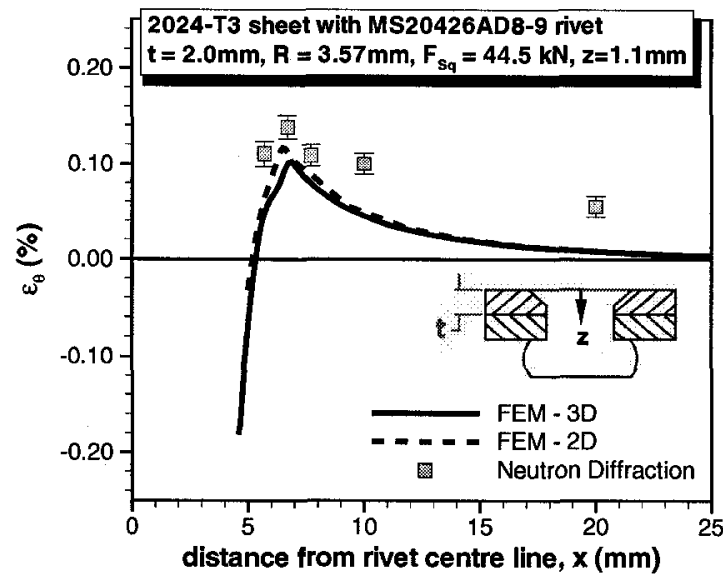

(a) $F_{S q}=44.5 \mathrm{kN}$

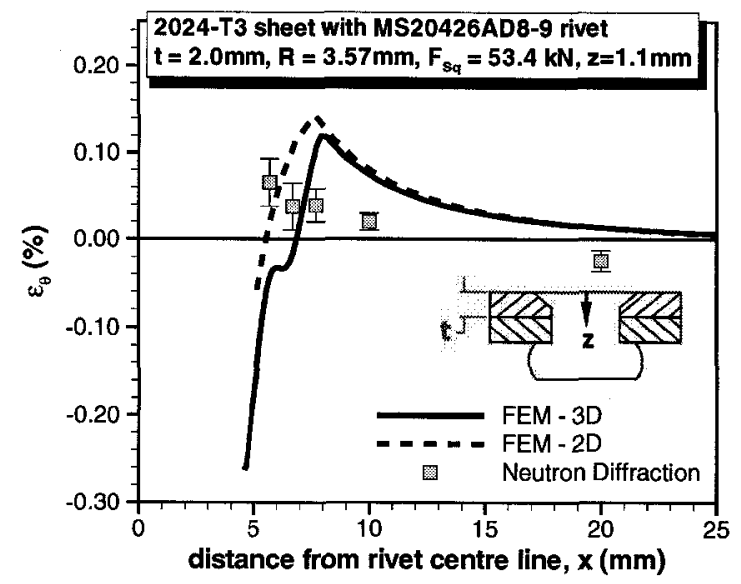

(b) $F_{S q}=53.4 \mathrm{kN}$

Figure B.4: Comparison of outer sheet residual tangential strains

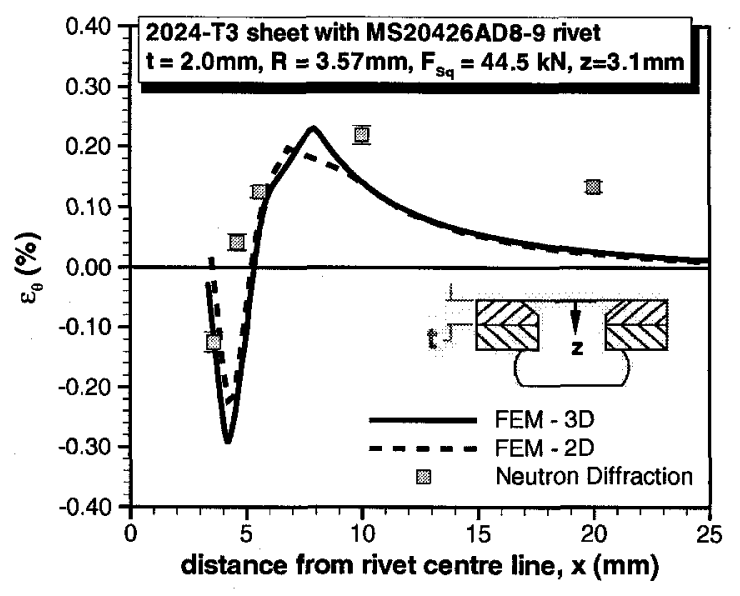

(a) $F_{S q}=44.5 \mathrm{kN}$

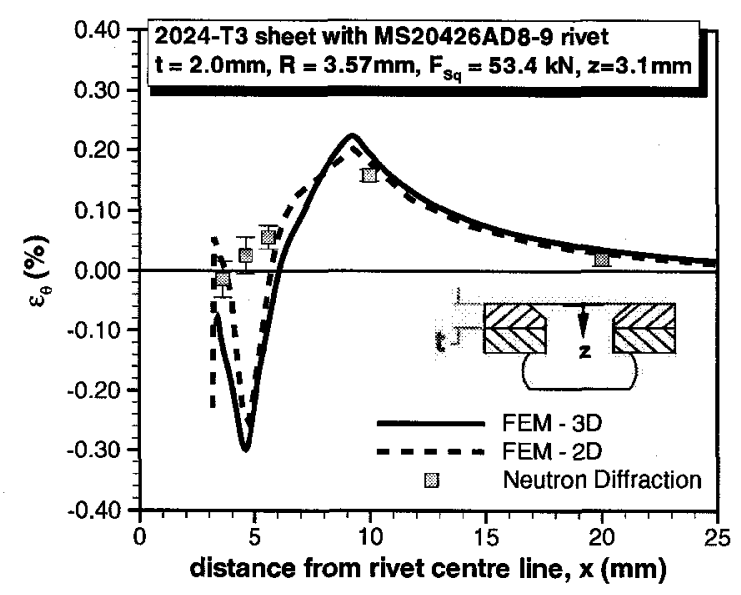

(b) $F_{S q}=53.4 \mathrm{kN}$

Figure B.5: Comparison of inner sheet residual tangential strains 


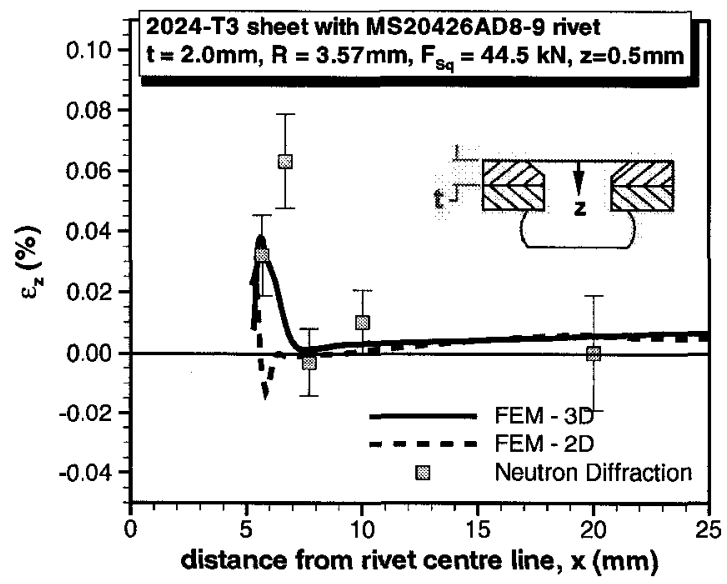

(a) $F_{S q}=44.5 \mathrm{kN}$

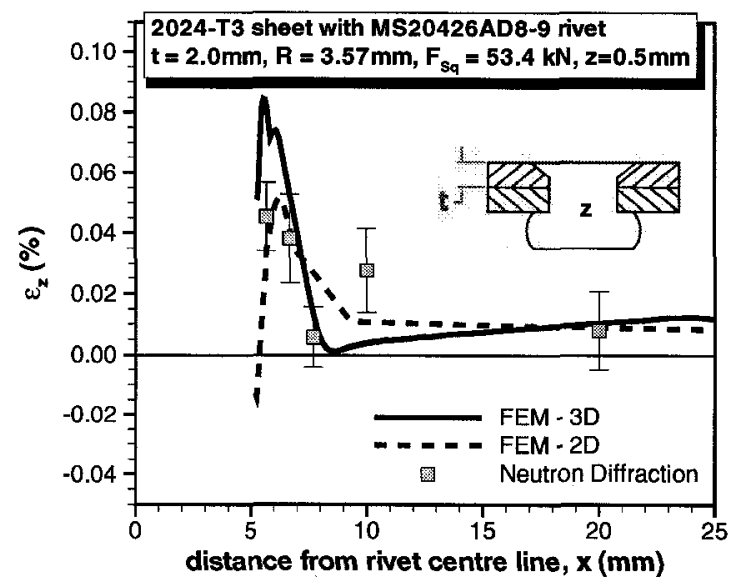

(b) $F_{S q}=53.4 \mathrm{kN}$

Figure B.6: Comparison of outer sheet residual clamping strains

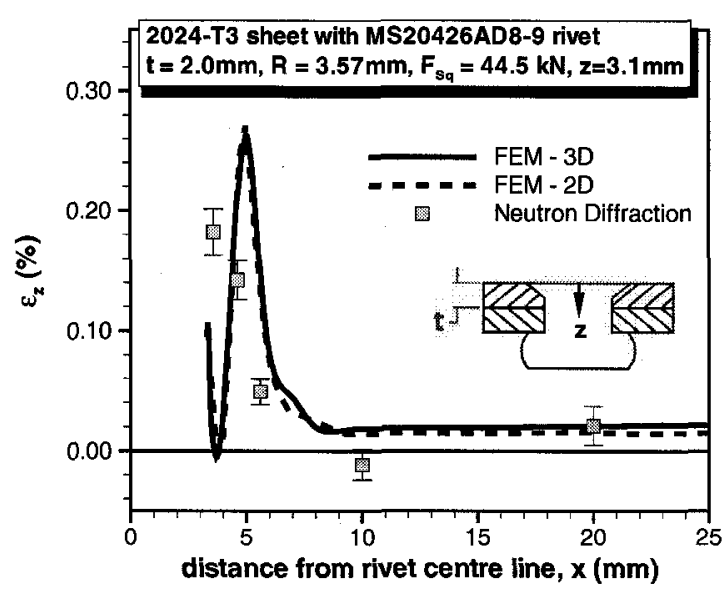

(a) $F_{S q}=44.5 \mathrm{kN}$

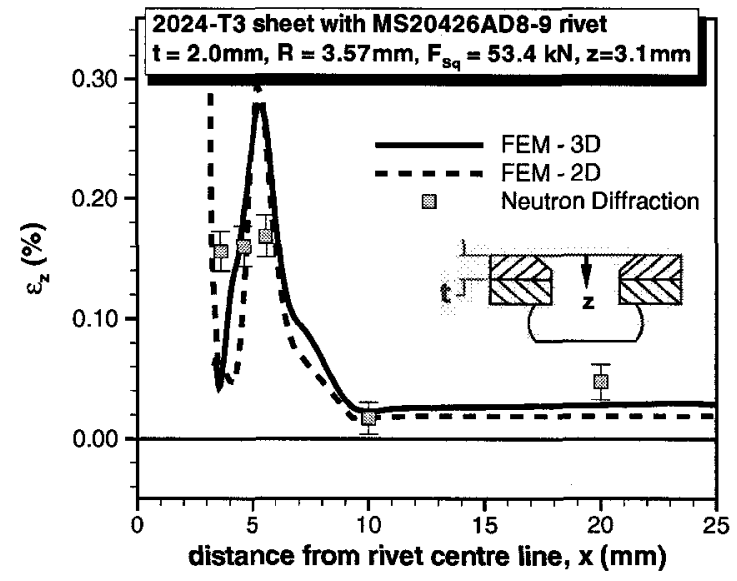

(b) $F_{S q}=53.4 \mathrm{kN}$

Figure B.7: Comparison of inner sheet residual clamping strains 


\section{Appendix C}

\section{Rivet Installation Finite Element Data}

The formation of residual stresses during rivet installation were investigated using a 3 dimension finite element model. This model is shown schematically in Figure C.1 and is described in detail in Chapter 4. The results from this model were also discussed in Chapter 4. Due to the large amounts of data obtained during the simulations, only a subset of the results was presented in order to support the overall conclusions from the study. This appendix contains supplementary contour plots of the residual stress data obtained during this study and serves as supporting data for the discussion and conclusions in Chapter 4 .

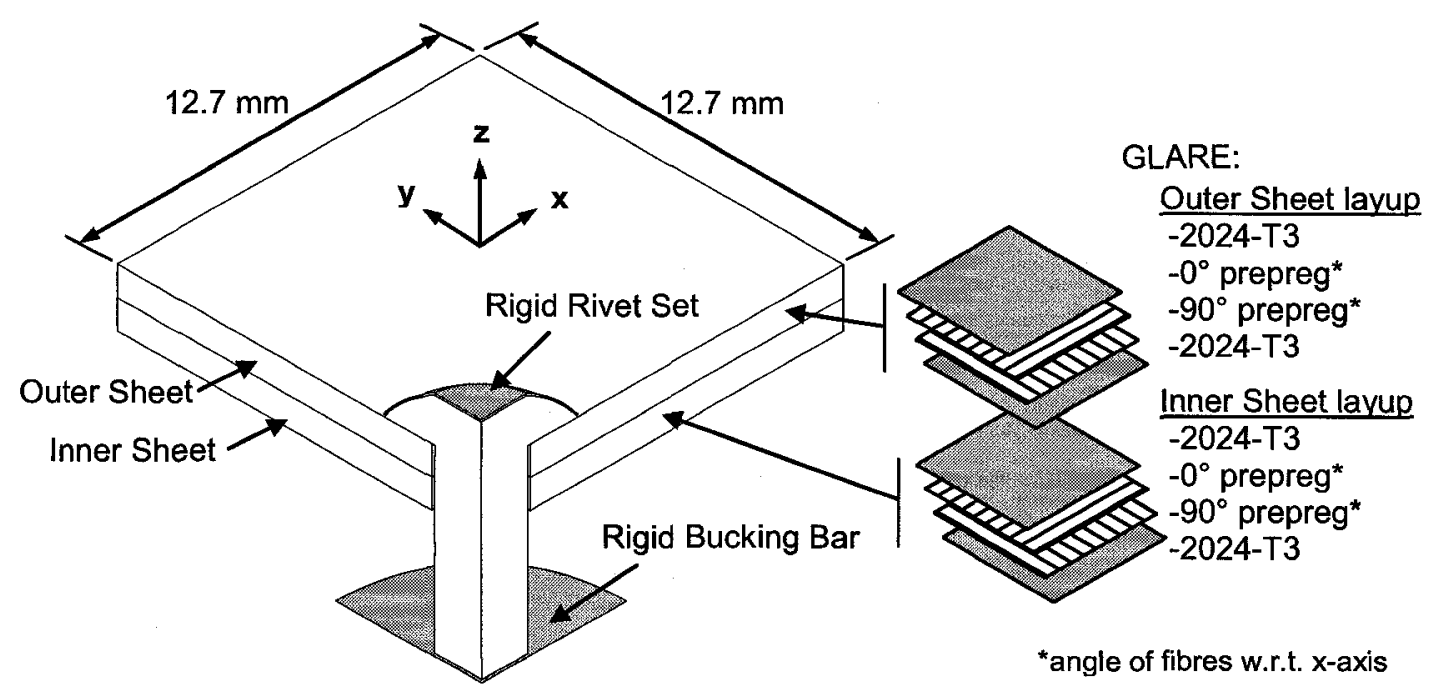

Figure C.1: Schematic of rivet installation finite element model 

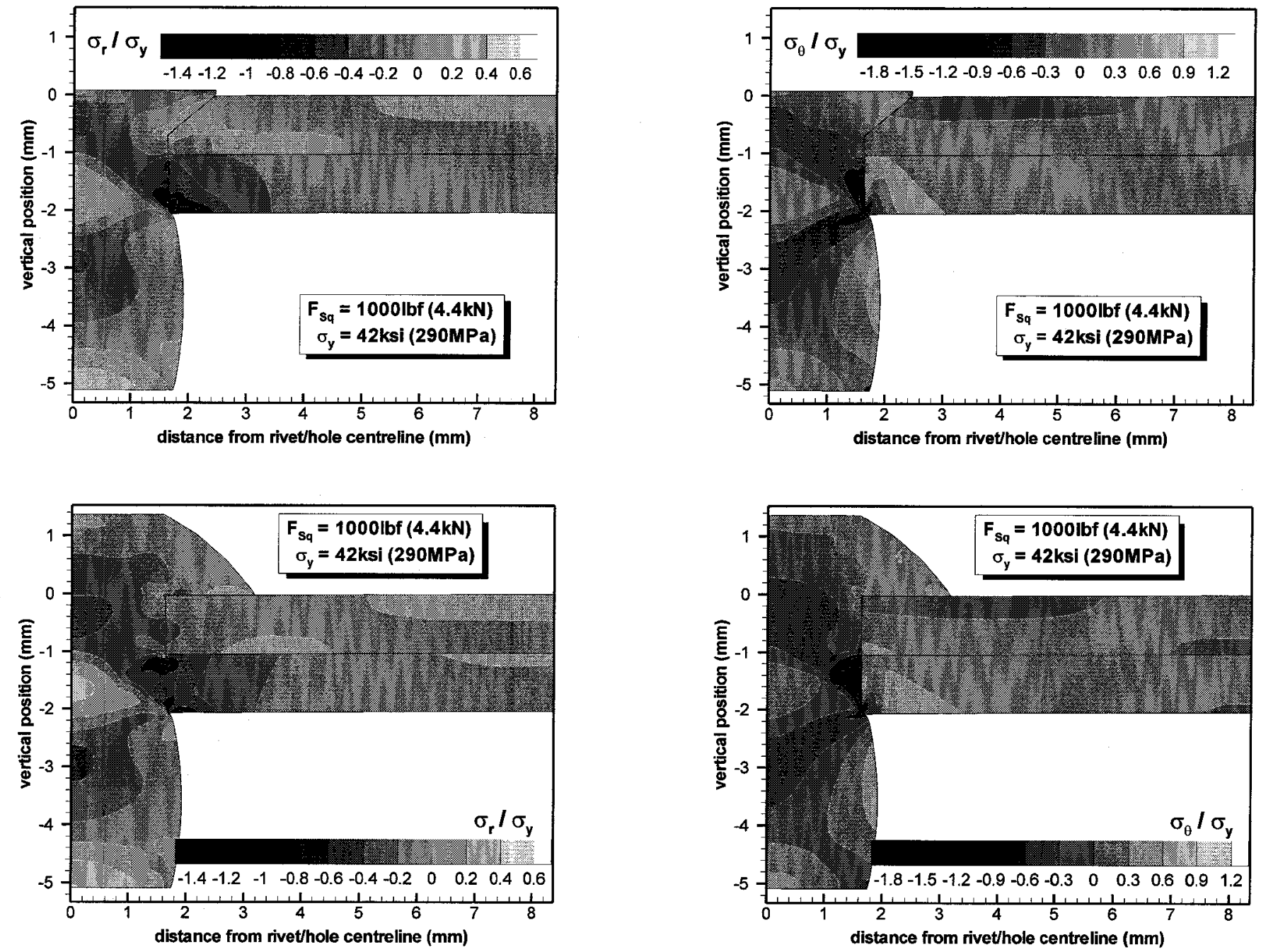

Figure C.2: Residual stress results for NAS1097AD4-4 and MS20470AD4-4 rivets installed in 2024-T3 sheet with $F_{S q}=1000 l b f$ 

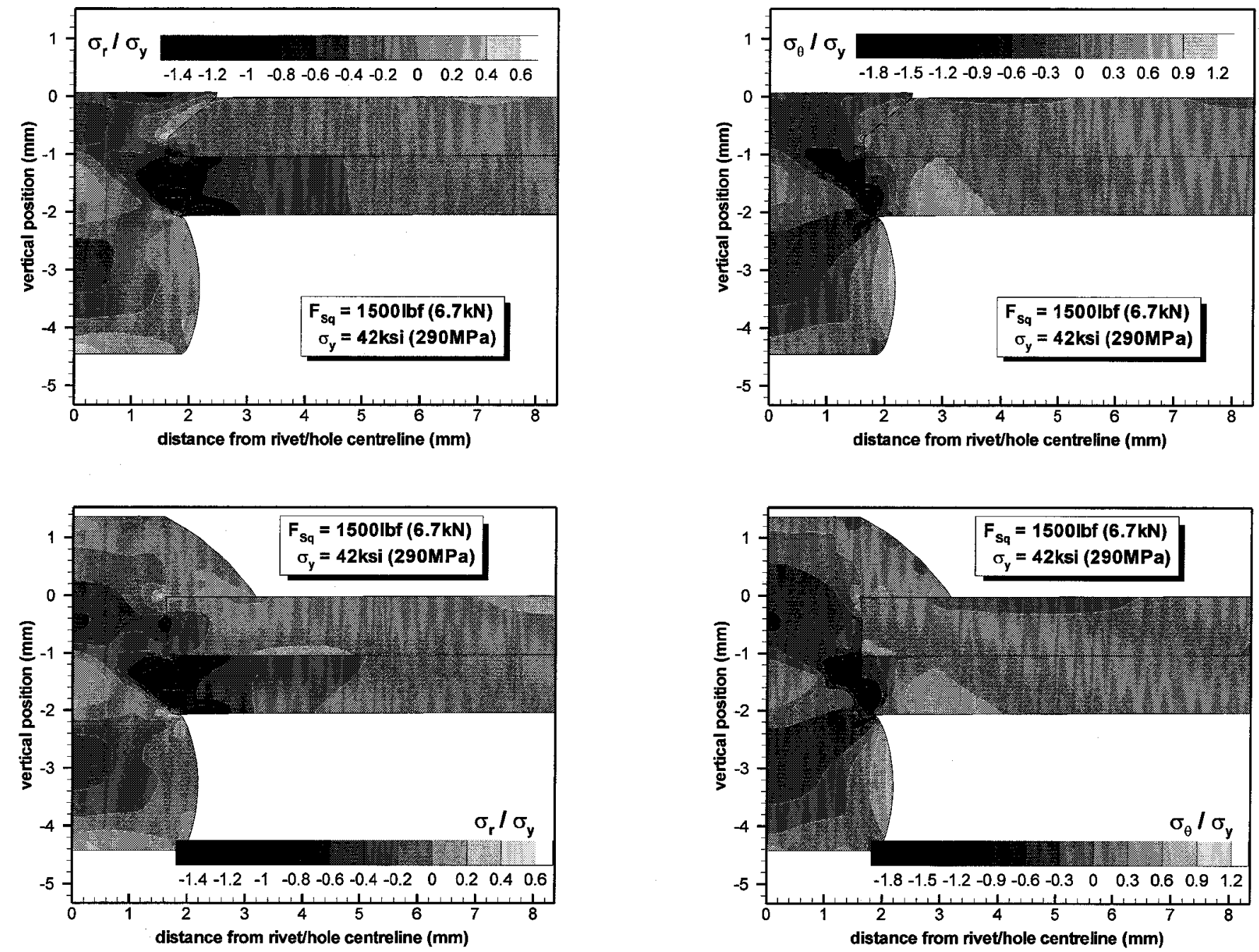

Figure C.3: Residual stress results for NAS1097AD4-4 and MS20470AD4-4 rivets installed in 2024-T3 sheet with $F_{S q}=1500 l b f$ 

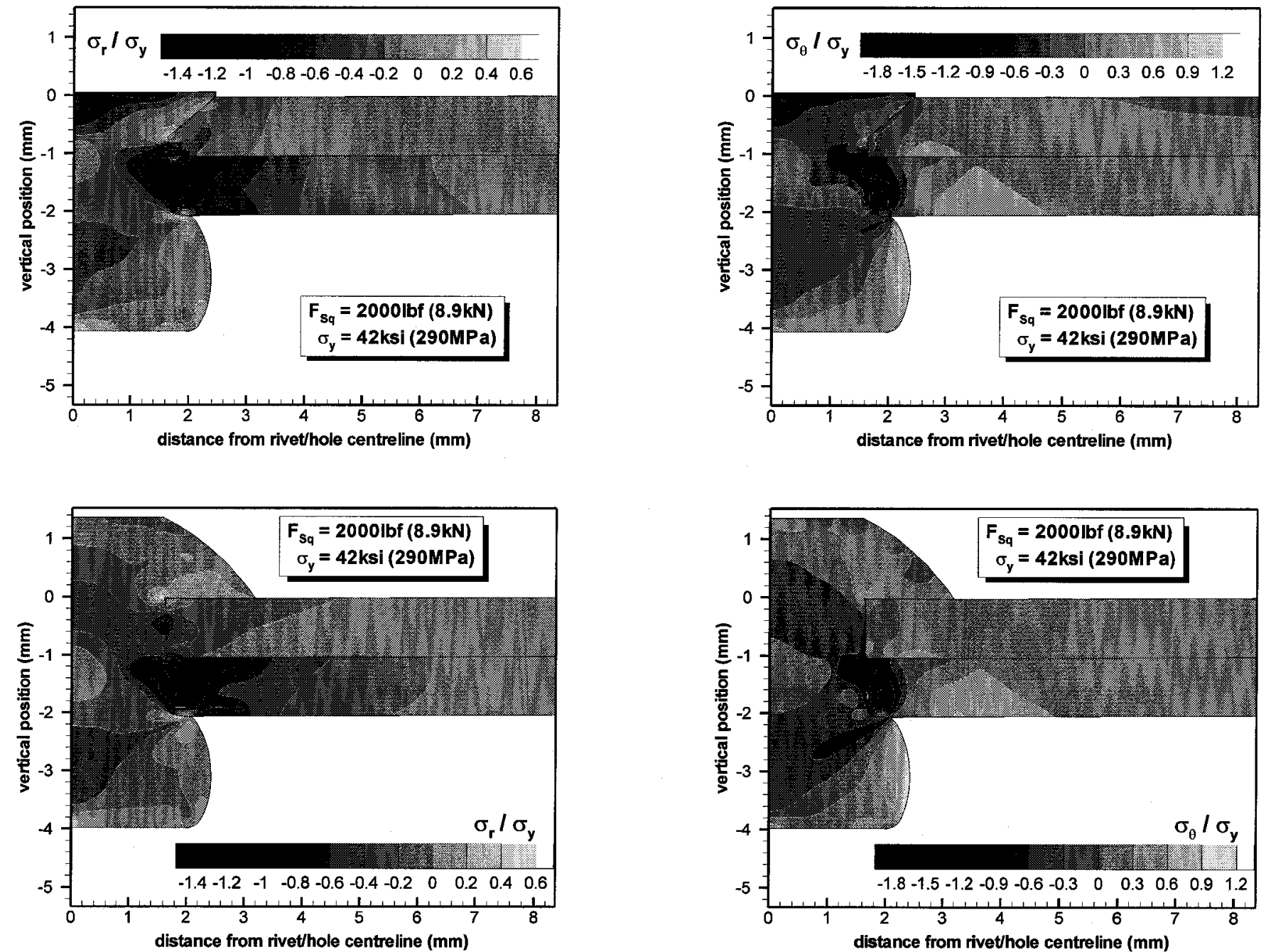

Figure C.4: Residual stress results for NAS1097AD4-4 and MS20470AD4-4 rivets installed in 2024-T3 sheet with $F_{S q}=2000 l b f$ 

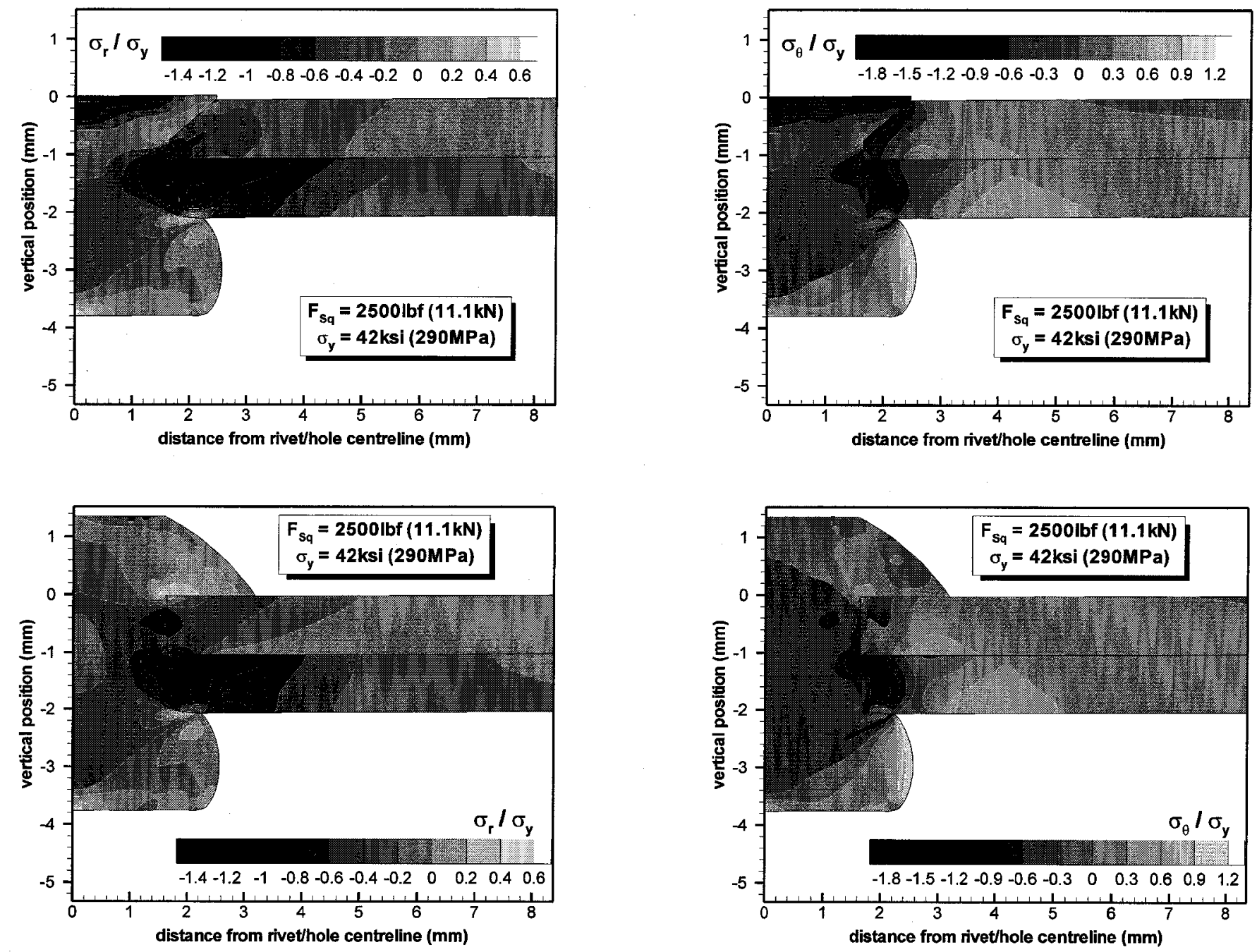

Figure C.5: Residual stress results for NAS1097AD4-4 and MS20470AD4-4 rivets installed in 2024-T3 sheet with $F_{S q}=2500 l b f$ 

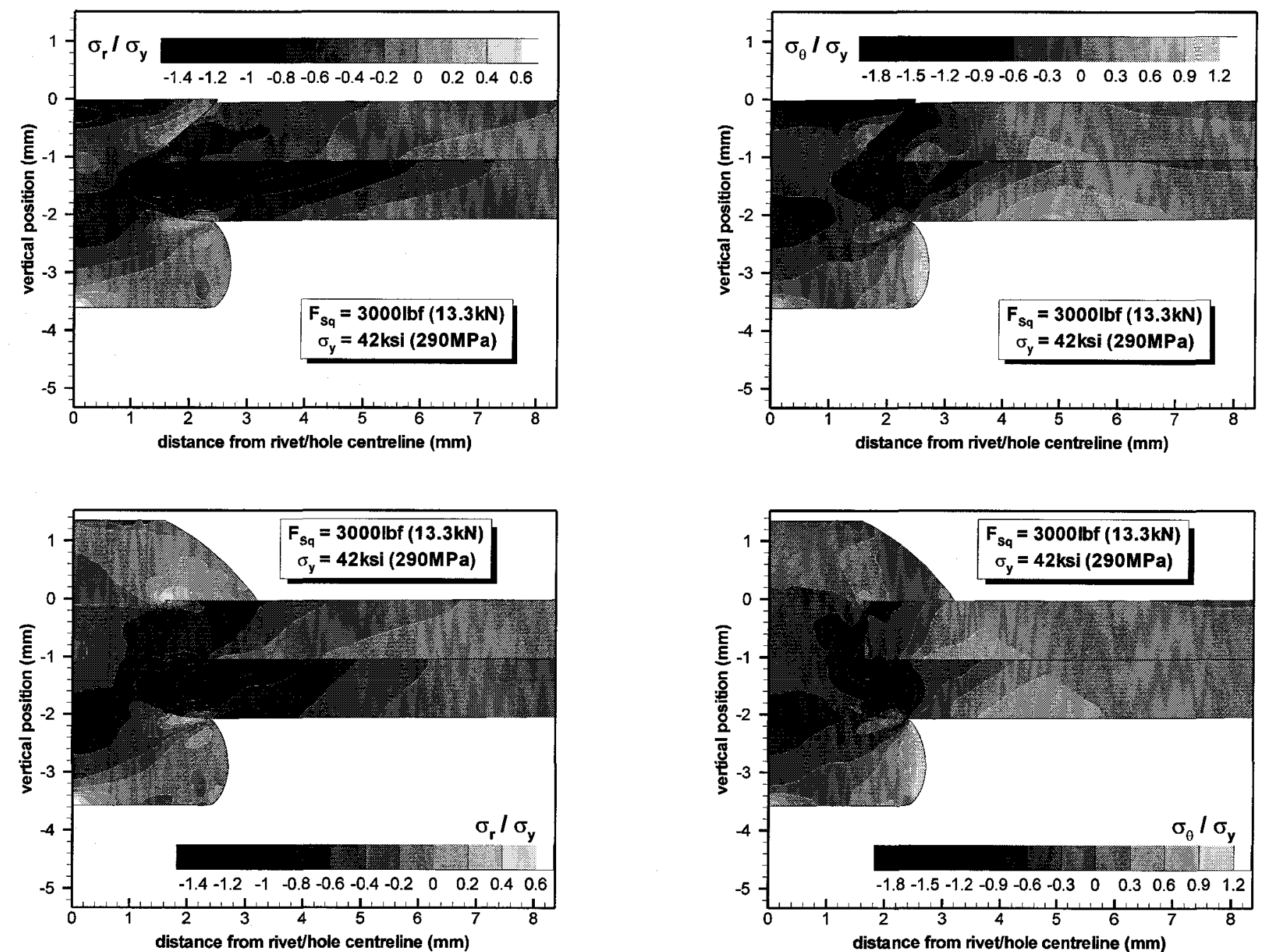

Figure C.6: Residual stress results for NAS1097AD4-4 and MS20470AD4-4 rivets installed in 2024-T3 sheet with $F_{S q}=3000 l b f$ 

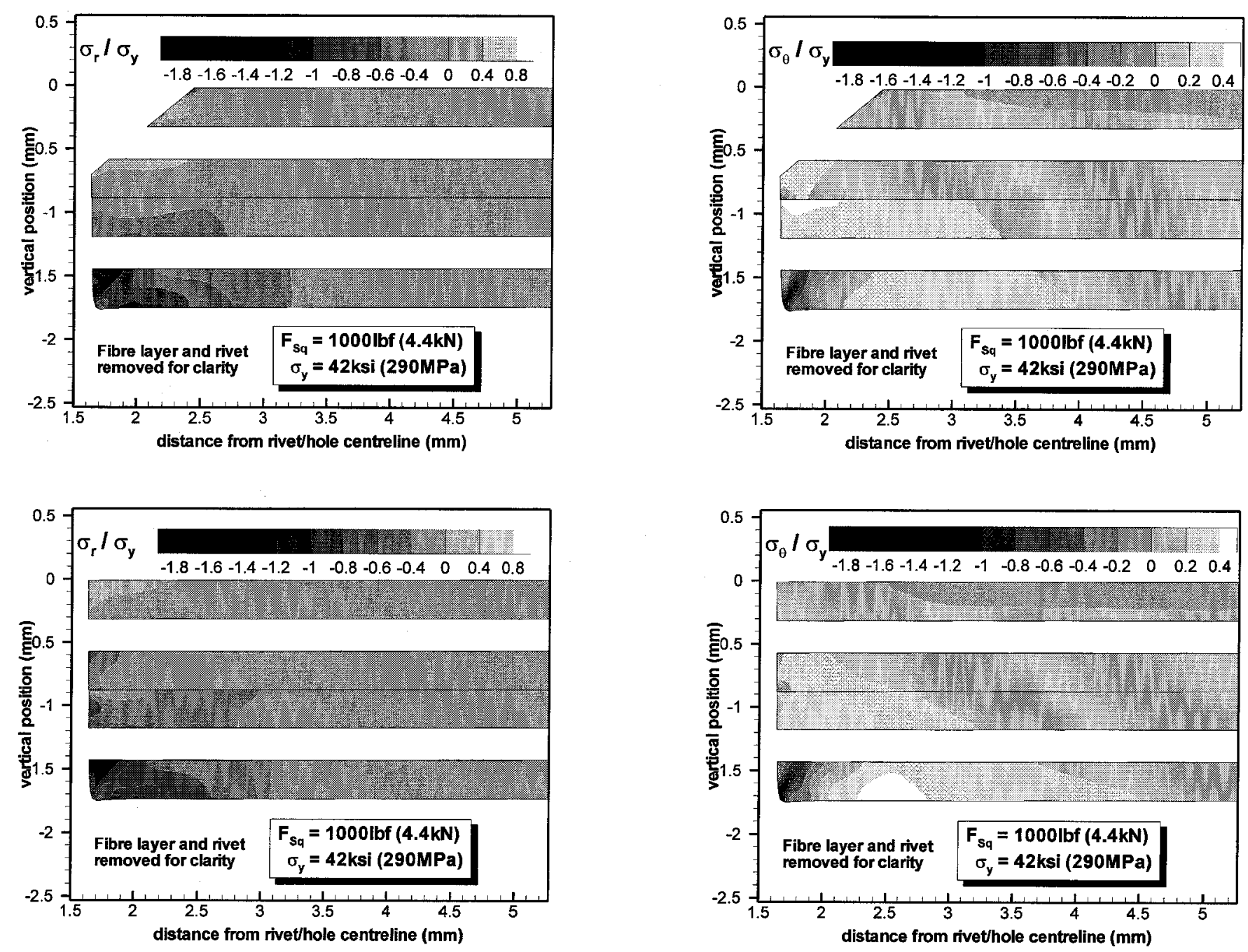

Figure C.7: Residual stress results for NAS1097AD4-4 and MS20470AD4-4 rivets installed in GLARE3-2/1-0.3 sheet with $F_{S q}=1000 l b f$ 

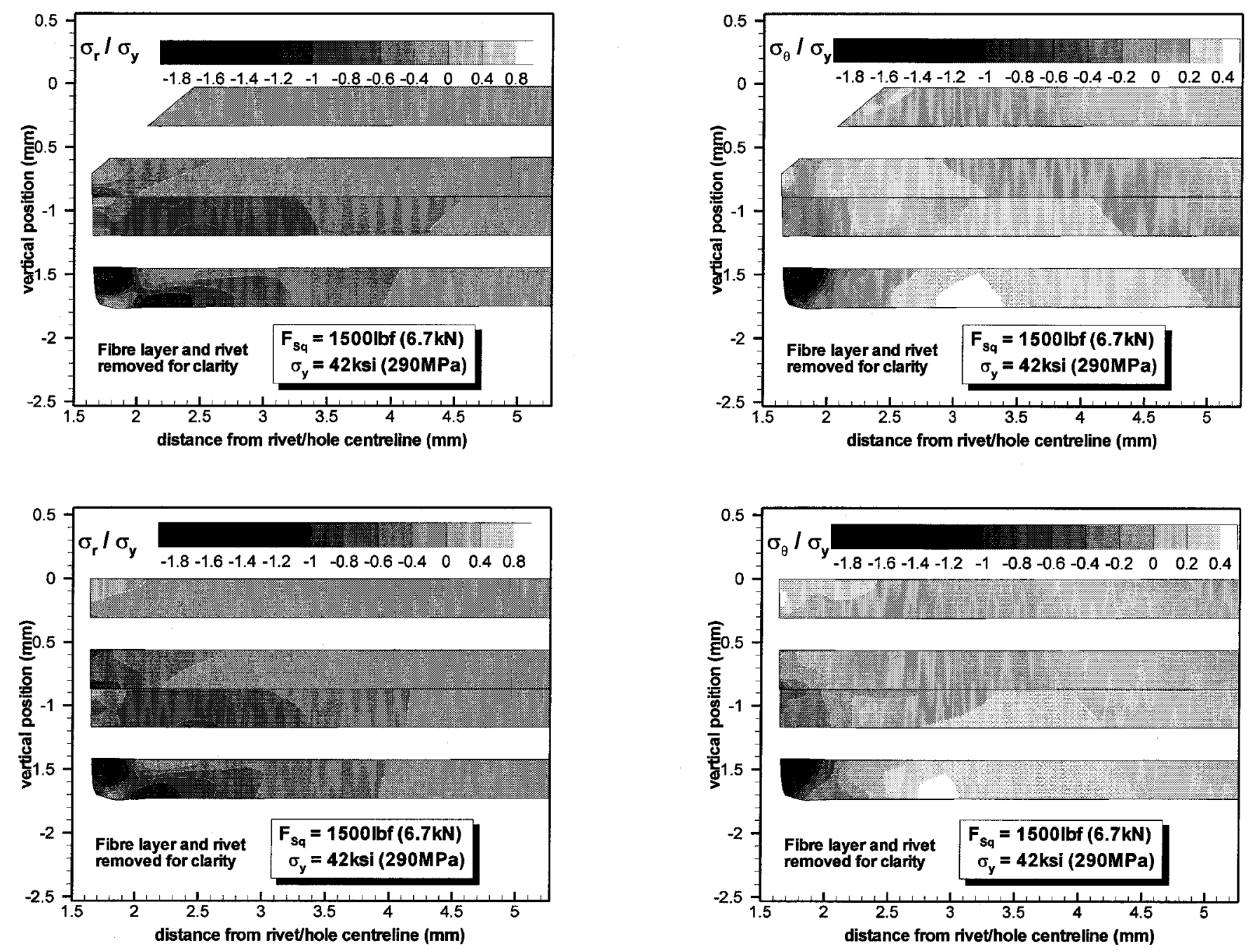

Figure C.8: Residual stress results for NAS1097AD4-4 and MS20470AD4-4 rivets installed in GLARE3-2/1-0.3 sheet with $F_{S q}=1500 l b f$ 

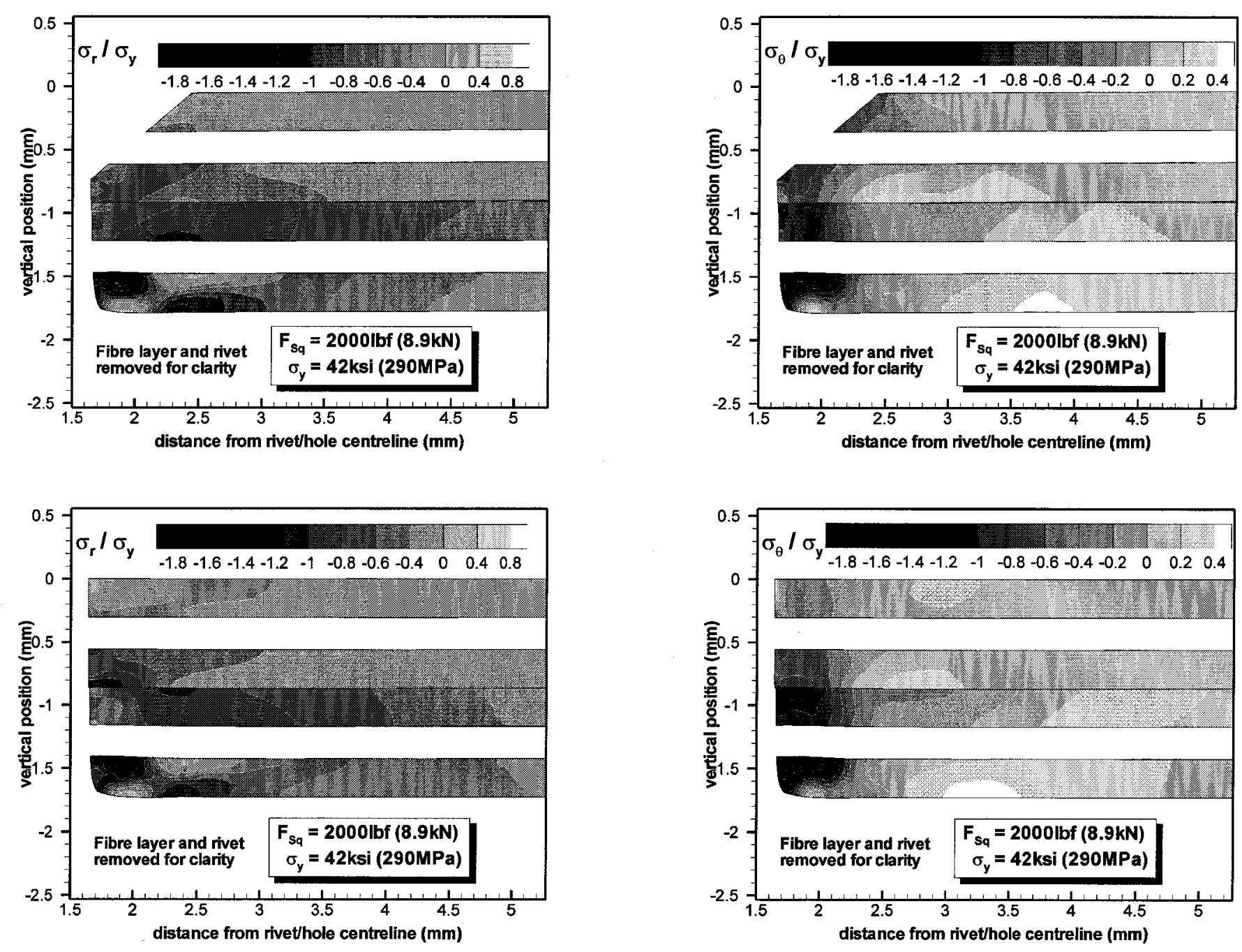

Figure C.9: Residual stress results for NAS1097AD4-4 and MS20470AD4-4 rivets installed in GLARE3-2/1-0.3 sheet with $F_{S q}=2000 l b f$ 

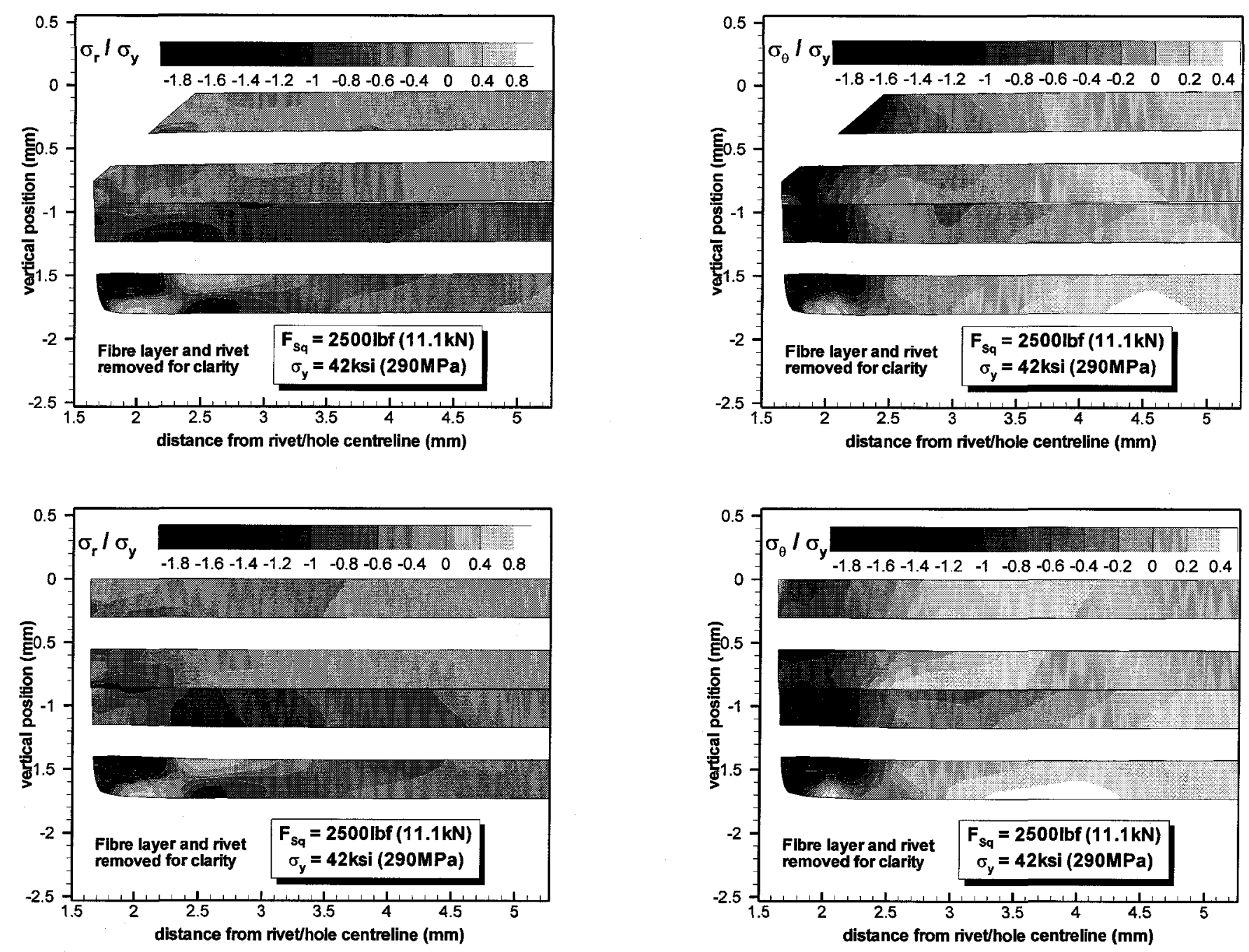

Figure C.10: Residual stress results for NAS1097AD4-4 and MS20470AD4-4 rivets installed in GLARE3-2/1-0.3 sheet with $F_{S q}=2500 l b f$ 

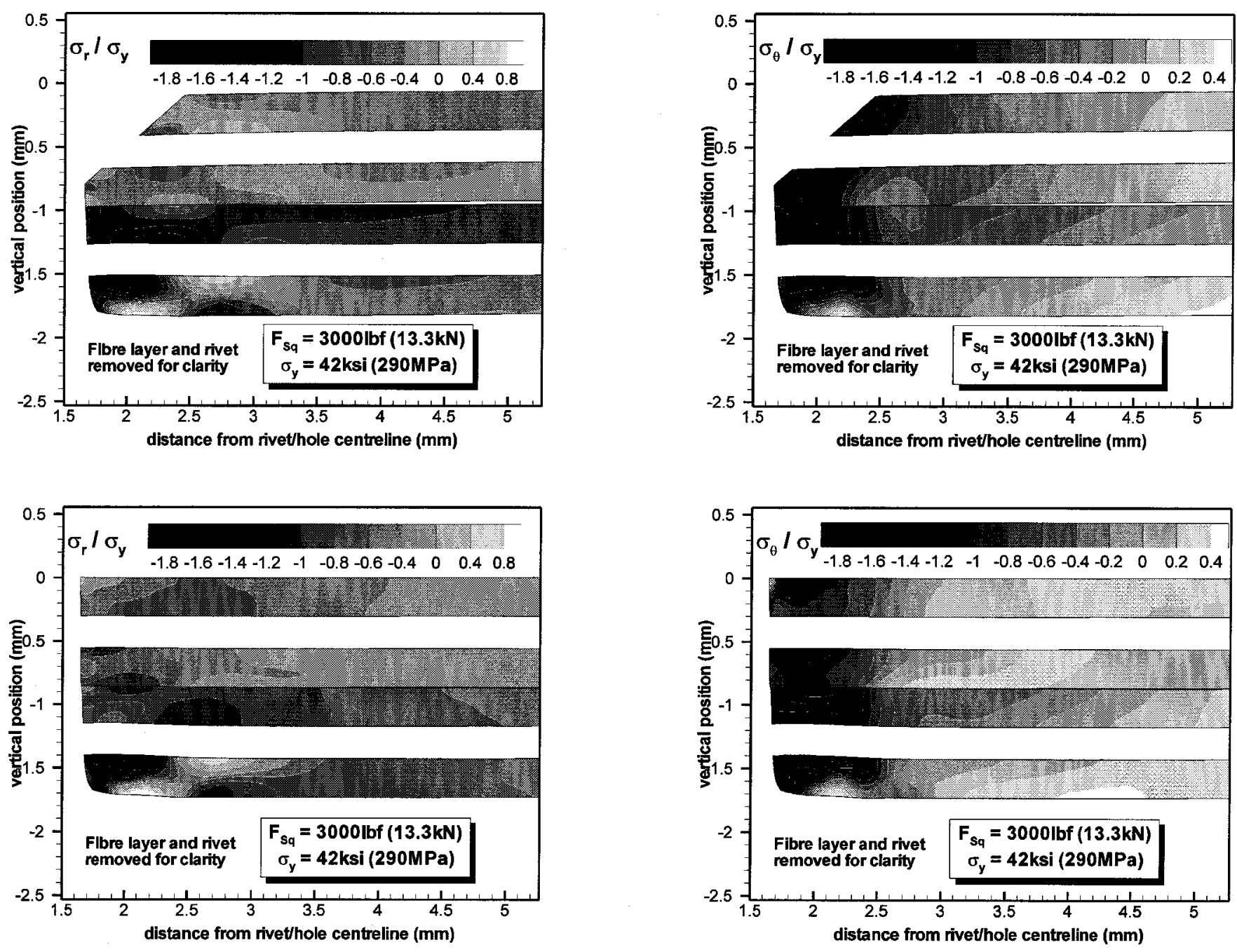

Figure C.11: Residual stress results for NAS1097AD4-4 and MS20470AD4-4 rivets installed in GLARE3-2/1-0.3 sheet with $F_{S q}=3000 l b f$ 


\section{Appendix D}

\section{Splice Loading Finite Element Data}

In Chapter 5, a finite element model used to investigate secondary bending in a loaded riveted splice was presented. For clarity and conciseness, only portions of the obtained results were presented in that chapter. This appendix provides supplementary contour plots of the finite element results from this study. Refer to Chapter 5 for details regarding the finite element model.

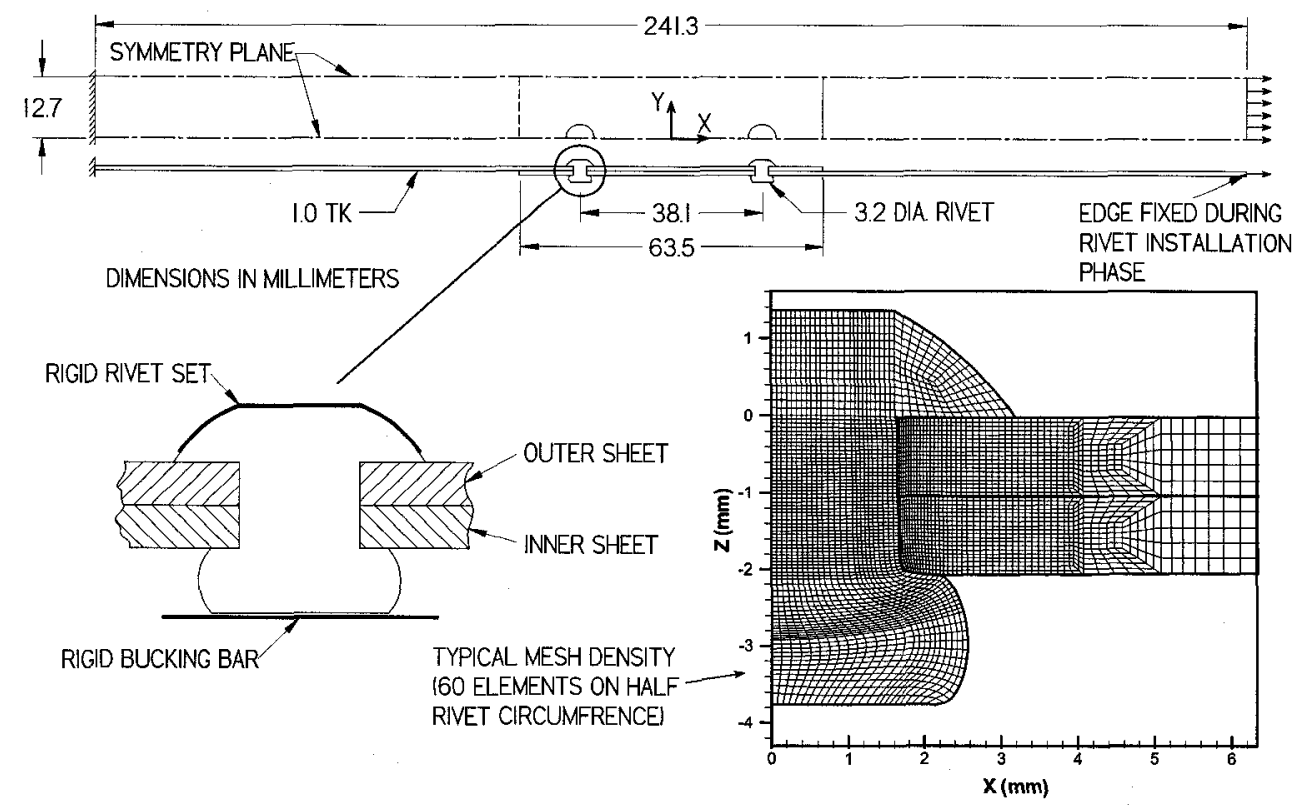

Figure D.1: Schematic of splice loading finite element model 


\section{4-T3 Results}
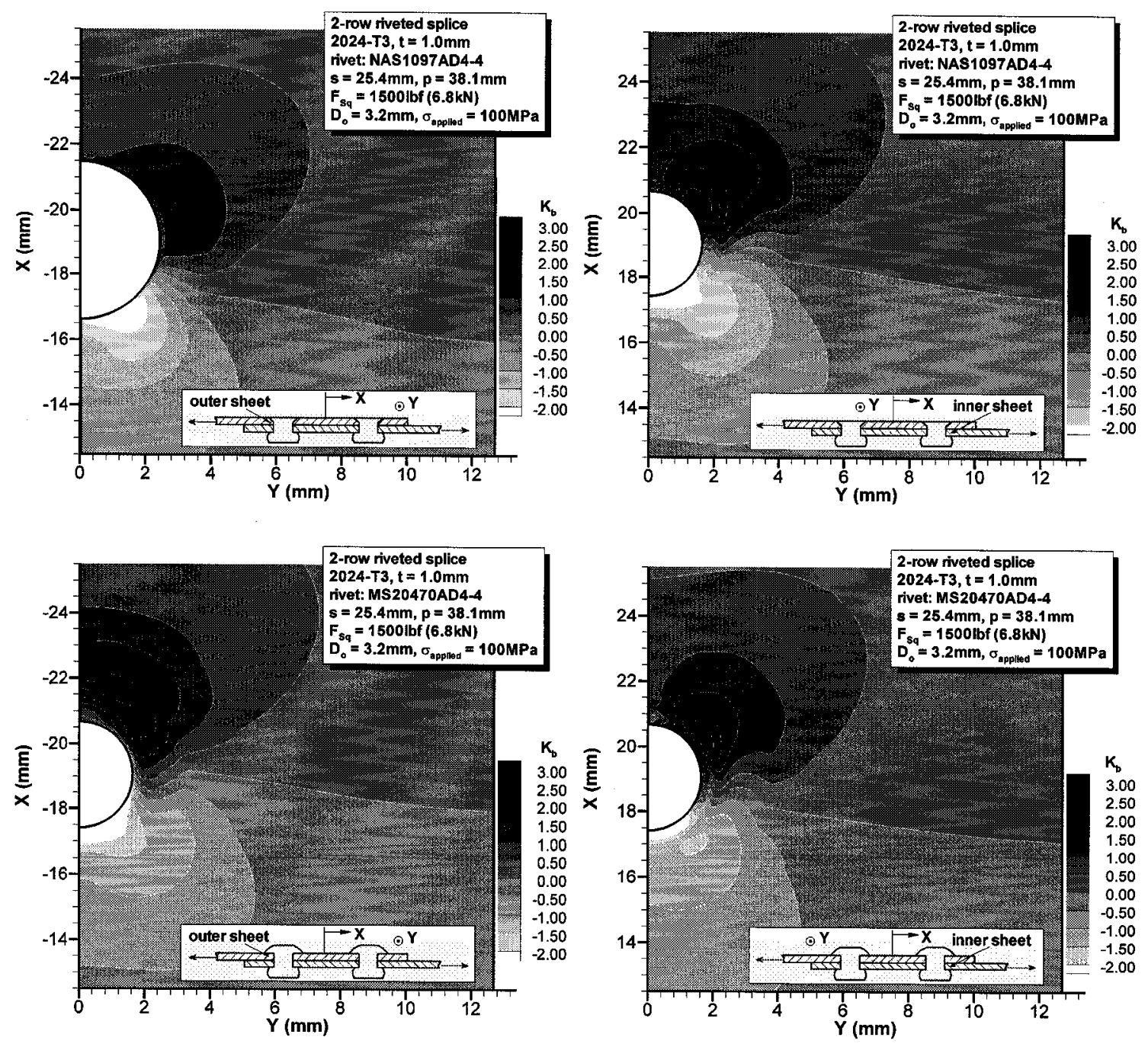

Figure D.2: Secondary bending factor results for 2024-T3 joints with NAS1097AD4-4 and MS20470AD4-4 rivets installed with $F_{S q}=1500 l b f$ 

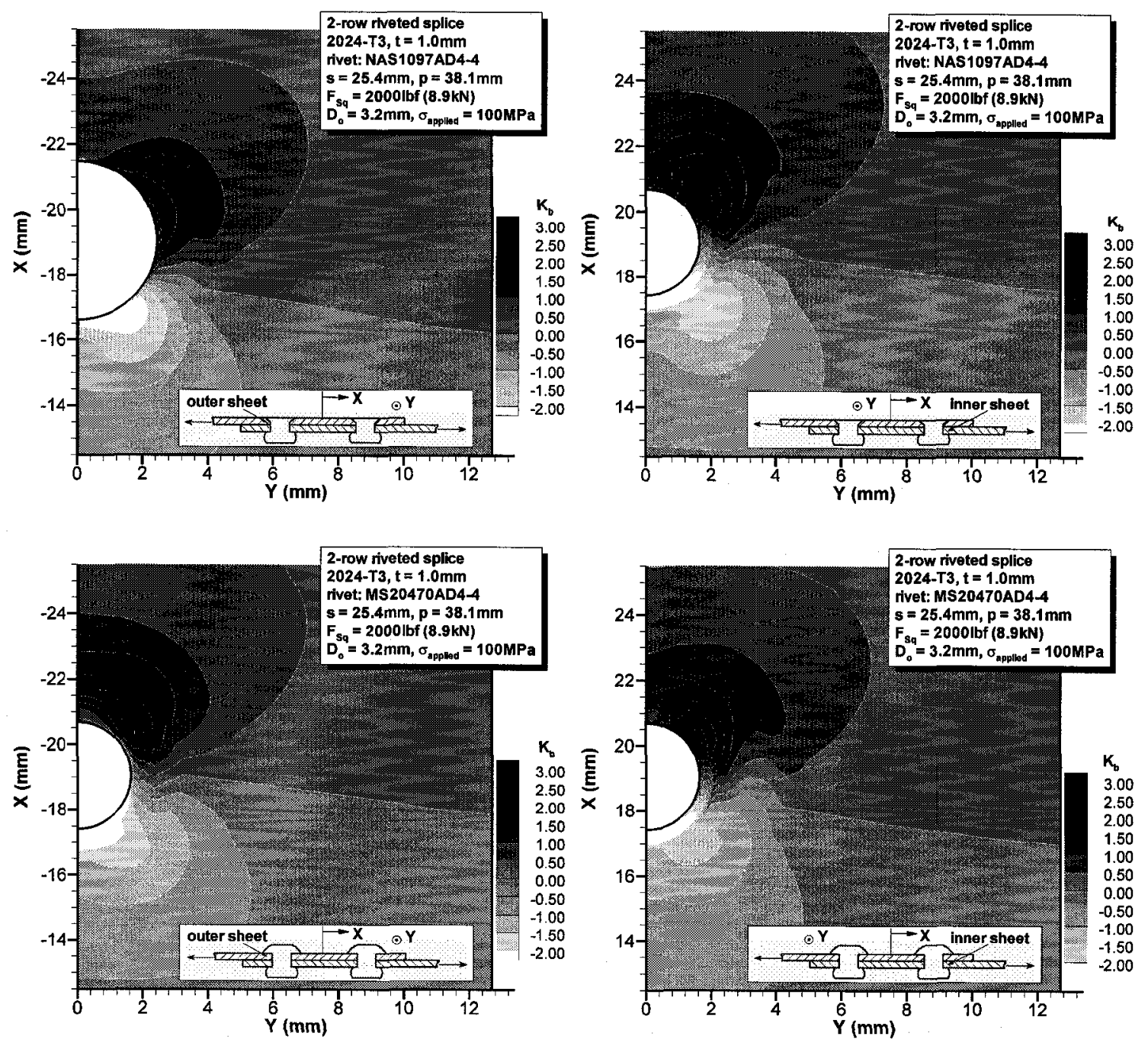

Figure D.3: Secondary bending factor results for 2024-T3 joints with NAS1097AD4-4 and MS20470AD4-4 rivets installed with $F_{S q}=2000 \mathrm{lbf}$ 

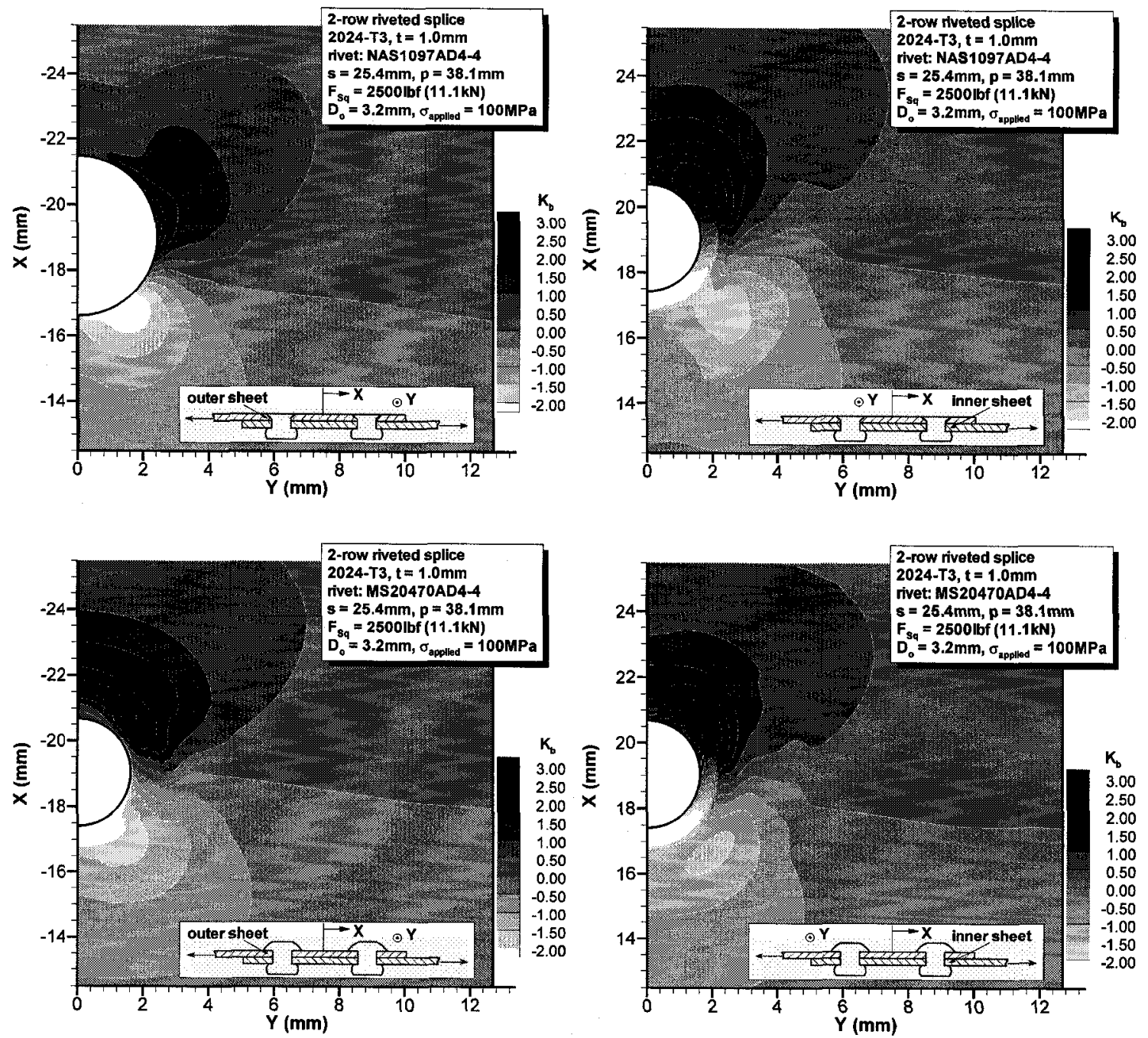

Figure D.4: Secondary bending factor results for 2024-T3 joints with NAS1097AD4-4 and MS20470AD4-4 rivets installed with $F_{S q}=2500 \mathrm{lbf}$ 

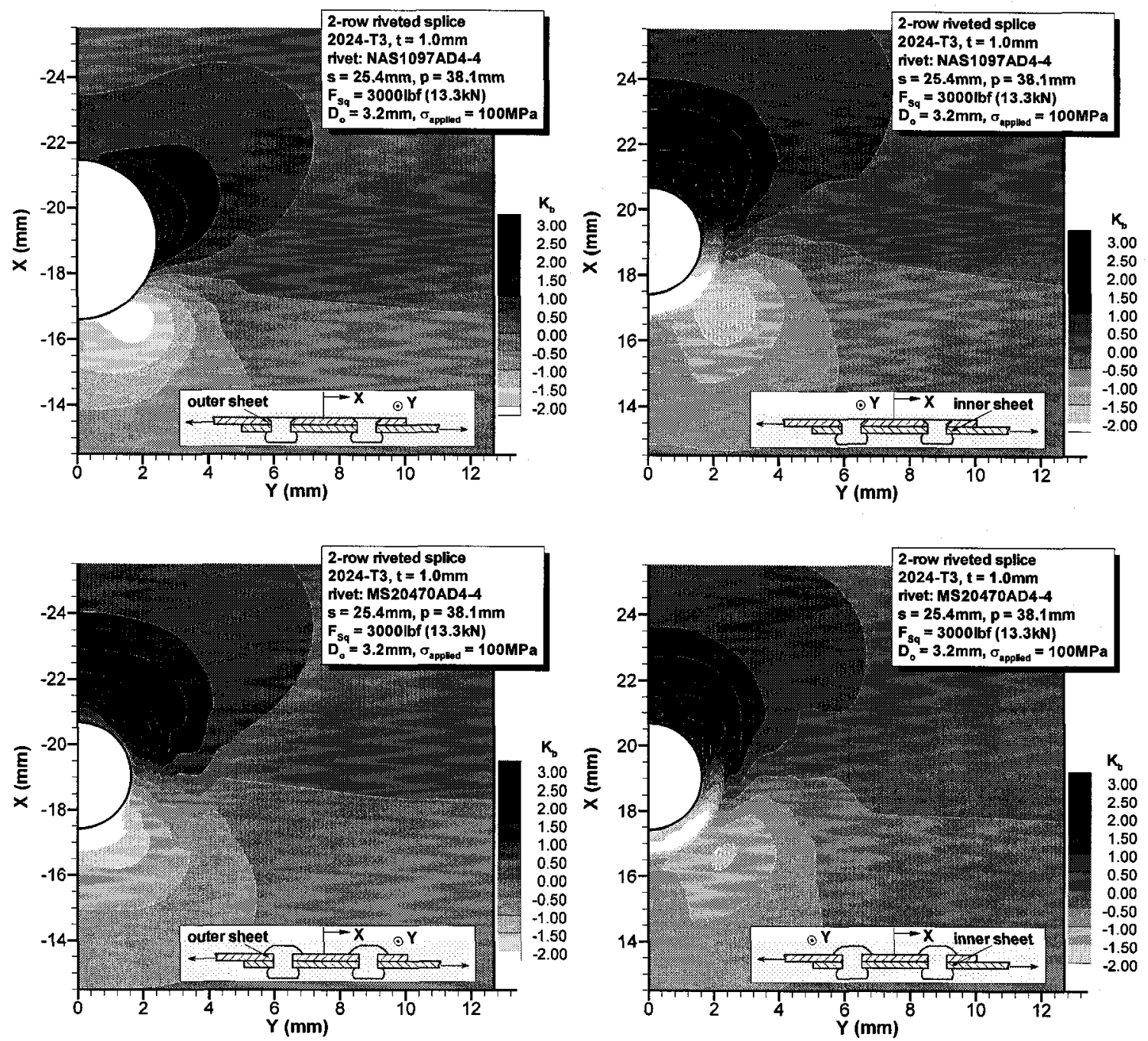

Figure D.5: Secondary bending factor results for 2024-T3 joints with NAS1097AD4-4 and MS20470AD4-4 rivets installed with $F_{S q}=3000 \mathrm{lbf}$ 


\section{GLARE3-2/1-0.3 Results}
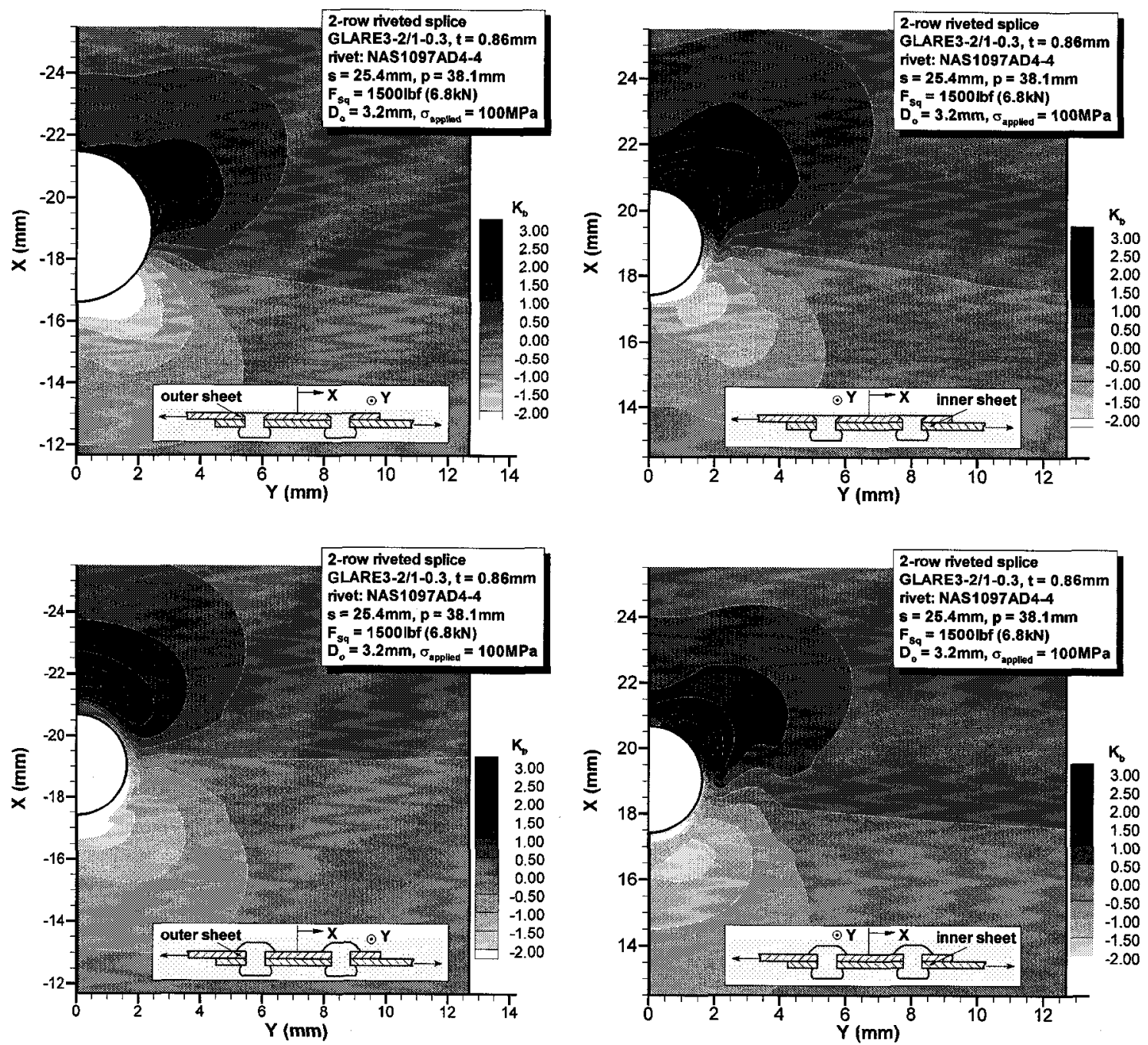

Figure D.6: Secondary bending factor results for GLARE joints with NAS1097AD4-4 and MS20470AD4-4 rivets installed with $F_{S q}=1500 \mathrm{lbf}$ 

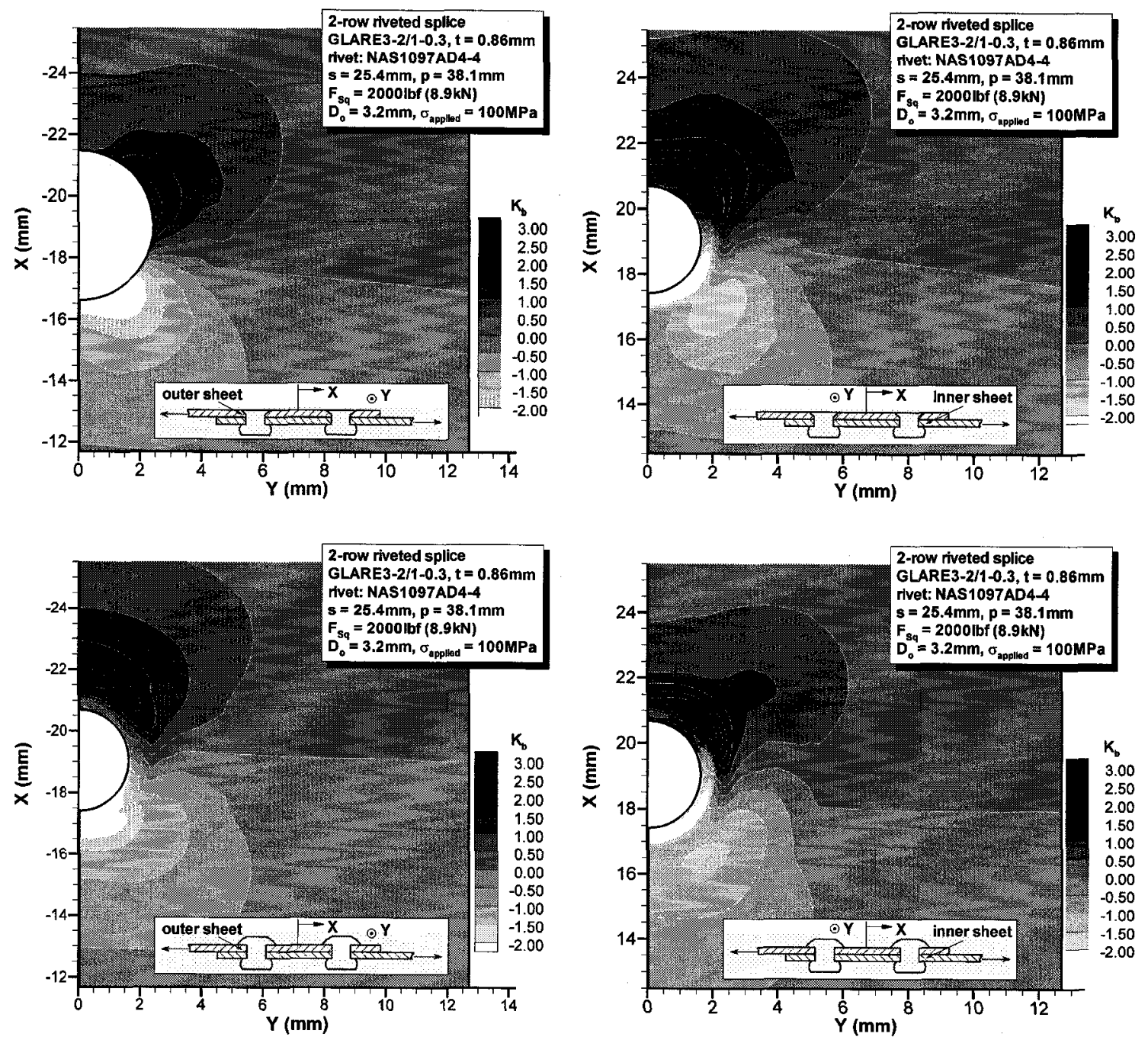

Figure D.7: Secondary bending factor results for GLARE joints with NAS1097AD4-4 and MS20470AD4-4 rivets installed with $F_{S q}=2000 \mathrm{lb} f$ 

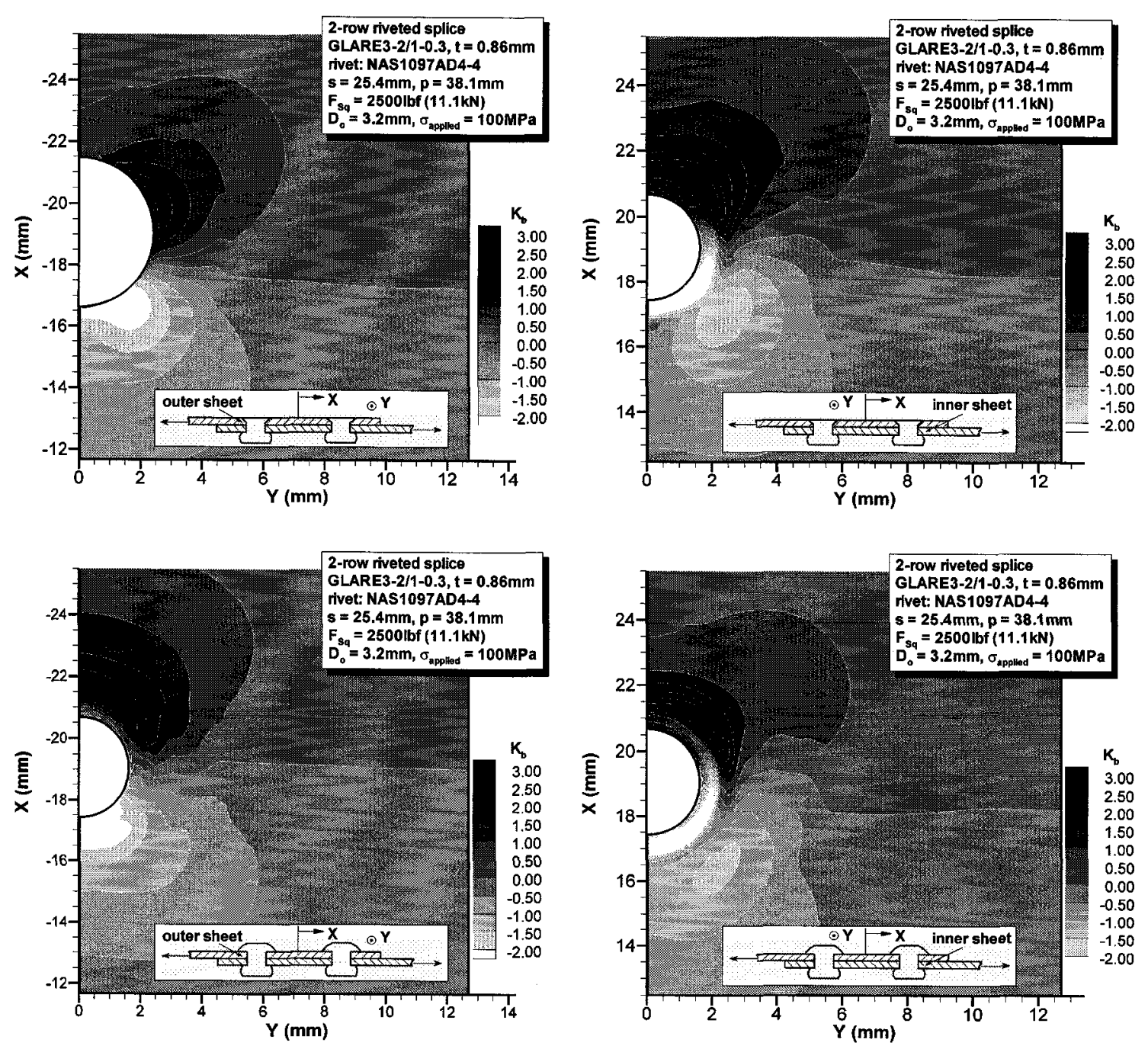

Figure D.8: Secondary bending factor results for GLARE joints with NAS1097AD4-4 and MS20470AD4-4 rivets installed with $F_{S q}=2500 l b f$ 

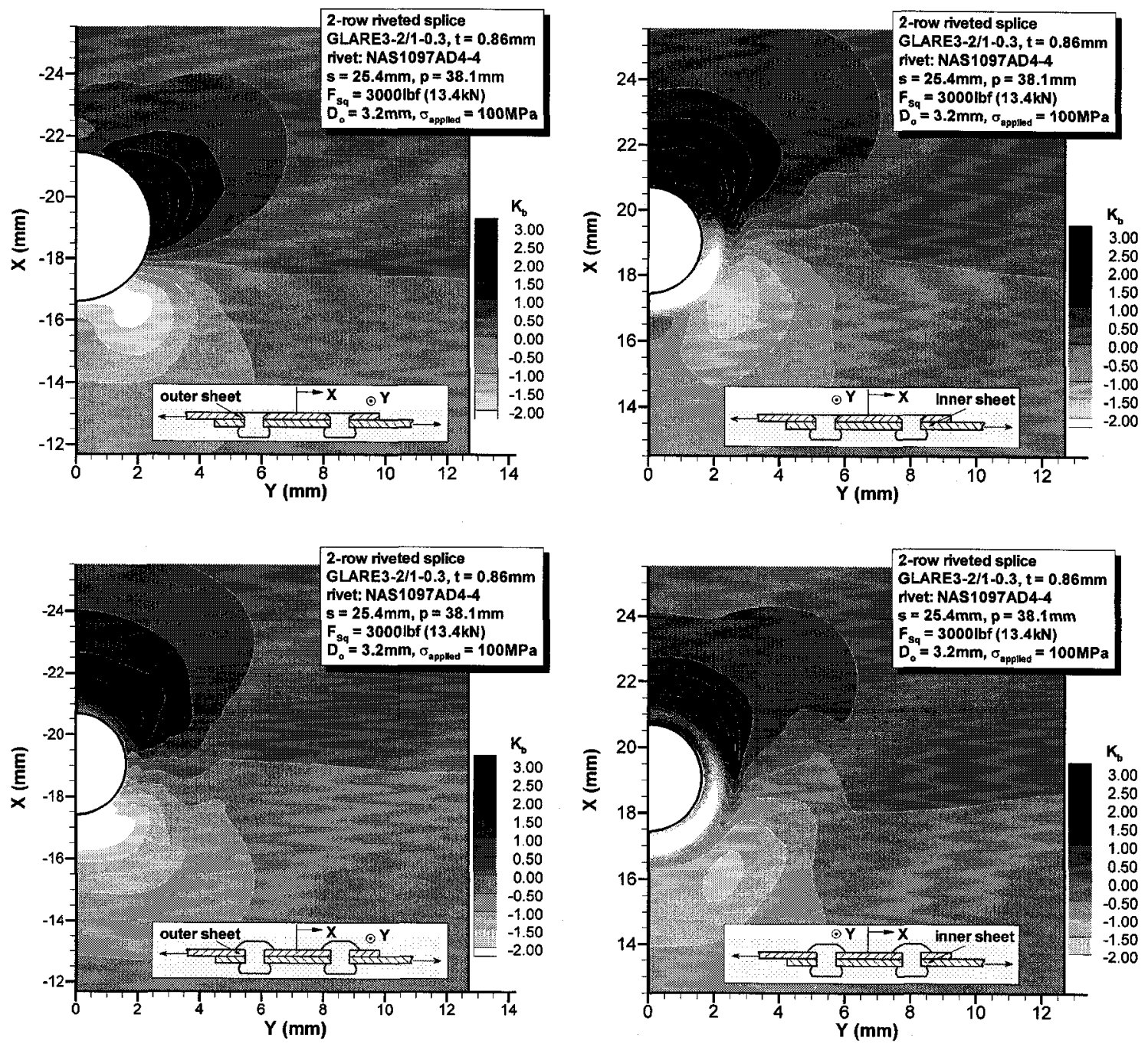

Figure D.9: Secondary bending factor results for GLARE joints with NAS1097AD4-4 and MS20470AD4-4 rivets installed with $F_{S q}=3000 l b f$ 


\section{Appendix E}

\section{Overall Fatigue Life Specimen Data}

In Chapter 6, the results from a fatigue test series completed in order to assess the effect of rivet installation on the overall fatigue life of a simple 2-row lap joint coupon were summarized. The specimen geometry and testing conditions used for this test series were described in Section 6.3. A detailed breakdown of the results are presented here. Fatigue life data is presented in the following section followed by a statistical analysis of the results by population. The specimen naming convention used to distinguish each test is summarized below:

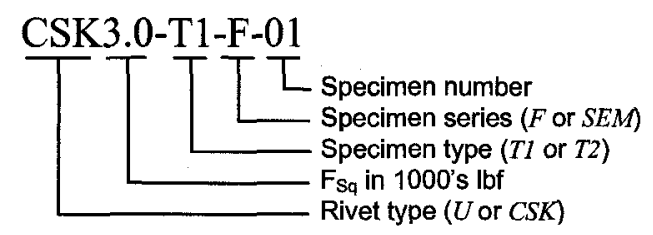

\section{Fatigue Life Data}

Table E.1: Type 1 coupon fatigue life results

\begin{tabular}{ccccc}
\hline \multirow{2}{*}{ Specimen Name } & \multirow{2}{*}{$F_{S q}(l b f)$} & \multirow{2}{*}{ Rivet Type } & \multicolumn{2}{c}{ Fatigue Life (cycles) } \\
& & & uncorrected & corrected \\
\hline CSK1.5-T1-F-01 & & & 112575 & 73260 \\
CSK1.5-T1-F-02 & 1500 & NAS1097-AD4-4 & 113246 & 73697 \\
CSK1.5-T1-F-03 & & & 110785 & 72095 \\
Continued on Next Page... & & & \\
\hline
\end{tabular}




\begin{tabular}{|c|c|c|c|c|}
\hline \multirow{2}{*}{ Specimen Name } & \multirow{2}{*}{$F_{S q}(l b f)$} & \multirow{2}{*}{ Rivet Type } & \multicolumn{2}{|c|}{ Fatigue Life (cycles) } \\
\hline & & & uncorrected & corrected \\
\hline CSK1.5-T1-F-04 & \multirow{3}{*}{1500} & \multirow{3}{*}{ NAS1097-AD4-4 } & 117585 & 76521 \\
\hline CSK1.5-T1-F-05 & & & 131785 & 85762 \\
\hline CSK1.5-T1-F-06 & & & 119230 & 77591 \\
\hline CSK2.0-T1-F-01 & \multirow{6}{*}{2000} & \multirow{6}{*}{ NAS1097-AD4-4 } & 145150 & 94459 \\
\hline CSK2.0-T1-F-02 & & & 125776 & 81851 \\
\hline CSK2.0-T1-F-03 & & & 138909 & 90398 \\
\hline CSK2.0-T1-F-04 & & & 166609 & 108424 \\
\hline CSK2.0-T1-F-05 & & & 176250 & 114698 \\
\hline CSK2.0-T1-F-06 & & & 146937 & 95622 \\
\hline CSK2.5-T1-F-01 & \multirow{10}{*}{2500} & \multirow{10}{*}{ NAS1097-AD4-4 } & 315482 & 205306 \\
\hline CSK2.5-T1-F-02 & & & 209943 & 136624 \\
\hline CSK2.5-T1-F-03 & & & 169095 & 110042 \\
\hline CSK2.5-T1-F-04 & & & 167804 & 109202 \\
\hline CSK2.5-T1-F-05 & & & 186745 & 121528 \\
\hline CSK2.5-T1-F-06 & & & 179731 & 116963 \\
\hline CSK2.5-T1-F-07 & & & 156222 & 101664 \\
\hline CSK2.5-T1-F-08 & & & 148191 & 96438 \\
\hline CSK2.5-T1-F-09 & & & 199730 & 129978 \\
\hline CSK2.5-T1-F-10 & & & 235816 & 153462 \\
\hline CSK3.0-T1-F-01 & \multirow{9}{*}{3000} & \multirow{9}{*}{ NAS1097-AD4-4 } & 354778 & 230879 \\
\hline CSK3.0-T1-F-02 & & & 385022 & 250560 \\
\hline CSK3.0-T1-F-03 & & & 460623 & 299759 \\
\hline CSK3.0-T1-F-04 & & & 318723 & 207415 \\
\hline CSK3.0-T1-F-05 & & & 333519 & 217044 \\
\hline CSK3.0-T1-F-06 & & & 231978 & 150964 \\
\hline CSK3.0-T1-F-07 & & & 377327 & 245553 \\
\hline CSK3.0-T1-F-08 & & & 305852 & 199039 \\
\hline CSK3.0-T1-F-09 & & & 376787 & 245201 \\
\hline U1.5-T1-F-01 & \multirow{4}{*}{1500} & \multirow{4}{*}{ MS20470-AD4-4 } & 238375 & 155127 \\
\hline $\mathrm{U} 1.5-\mathrm{T} 1-\mathrm{F}-02$ & & & 230713 & 150141 \\
\hline U1.5-T1-F-03 & & & 199742 & 129986 \\
\hline U1.5-T1-F-04 & & & 201293 & 130995 \\
\hline U2.0-T1-F-01 & \multirow{2}{*}{2000} & \multirow{2}{*}{ MS20470-AD4-4 } & 218608 & 142263 \\
\hline U2.0-T1-F-02 & & & 215029 & 139934 \\
\hline Continued on Next & age... & & & \\
\hline
\end{tabular}




\begin{tabular}{ccccc}
\hline \multirow{2}{*}{ Specimen Name } & \multirow{2}{*}{$F_{S q}(l b f)$} & \multirow{2}{*}{ Rivet Type } & \multicolumn{2}{c}{ Fatigue Life (cycles) } \\
& & & uncorrected & corrected \\
\hline U2.0-T1-F-03 & \multirow{2}{*}{2000} & MS20470-AD4-4 & 251819 & 163876 \\
U2.0-T1-F-04 & & & 215950 & 140534 \\
\hline U2.5-T1-F-01 & & & 263379 & 171399 \\
U2.5-T1-F-02 & \multirow{2}{*}{2500} & MS20470-AD4-4 & 272024 & 177025 \\
U2.5-T1-F-03 & & & 270771 & 176209 \\
U2.5-T1-F-04 & & & 201855 & 131361 \\
\hline U3.0-T1-F-01 & & & 334889 & 217935 \\
U3.0-T1-F-02 & & & 279462 & 181865 \\
U3.0-T1-F-03 & \multirow{2}{*}{3000} & MS20470-AD4-4 & 305042 & 198512 \\
U3.0-T1-F-04 & & & 324484 & 211164 \\
U3.0-T1-F-05 & & & 356540 & 232025 \\
\hline
\end{tabular}

Table E.2: Type 2 coupon fatigue life results

\begin{tabular}{ccccc}
\hline \multirow{2}{*}{ Specimen Name } & \multirow{2}{*}{$F_{S q}(l b f)$} & \multirow{2}{*}{ Rivet Type } & \multicolumn{2}{c}{ Fatigue Life (cycles) } \\
& & & 137186 & 89276 \\
CSK1.5-T2-F-01 & \multirow{2}{*}{ corrected } \\
CSK1.5-T2-F-02 & \multirow{2}{*}{ NAS1097-AD4-4 } & 158700 & 103277 \\
CSK1.5-T2-F-03 & & & 188551 & 122703 \\
\hline CSK2.0-T2-F-01 & & & 232073 & 151026 \\
CSK2.0-T2-F-02 & \multirow{2}{*}{2000} & NAS1097-AD4-4 & 299871 & 195147 \\
CSK2.0-T2-F-03 & & & 278612 & 181312 \\
\hline CSK2.5-T2-F-01 & & & 334457 & 217654 \\
CSK2.5-T2-F-02 & \multirow{2}{*}{2500} & NAS1097-AD4-4 & 306353 & 199365 \\
CSK2.5-T2-F-03 & & & 347230 & 225967 \\
\hline CSK3.0-T2-F-01 & & & 383752 & 249734 \\
CSK3.0-T2-F-02 & \multirow{2}{*}{3000} & NAS1097-AD4-4 & 521463 & 339352 \\
CSK3.0-T2-F-03 & & & 575886 & 374769 \\
\hline U1.5-T2-F-01 & & & 154045 & 100248 \\
U1.5-T2-F-02 & \multirow{2}{*}{1500} & MS20470-AD4-4 & 162852 & 105979 \\
U1.5-T2-F-03 & & & 166774 & 108531 \\
\hline Continued on Next Page... & & & \\
\hline
\end{tabular}




\begin{tabular}{ccccc}
\hline \multirow{2}{*}{ Specimen Name } & \multirow{2}{*}{$F_{S q}($ lbf $)$} & \multirow{2}{*}{ Rivet Type } & \multicolumn{2}{c}{ Fatigue Life (cycles) } \\
& & & 300170 & 195341 \\
U2.0-T2-F-01 & & & 262091 & 170561 \\
U2.0-T2-F-02 & \multirow{2}{*}{2000} & MS20470-AD4-4 & 390484 & 254115 \\
U2.0-T2-F-03 & & & 617377 & 401770 \\
\hline U2.5-T2-F-01 & & & $>700000$ & $>450000$ \\
U2.5-T2-F-02 & \multirow{2}{*}{2500} & MS20470-AD4-4 & $>700000$ & $>450000$ \\
U2.5-T2-F-03 & & & $>700000$ & $>450000$ \\
\hline U3.0-T2-F-01 & & & $>700000$ & $>450000$ \\
U3.0-T2-F-02 & \multirow{2}{*}{3000} & MS20470-AD4-4 & $>700000$ & $>450000$ \\
\hline U3.0-T2-F-03 & & &
\end{tabular}

\section{Confidence Intervals}

Confidence intervals provide a means of bounding an observed parameter of a population based on a limited sample of that population. In the current instance, confidence intervals are used to bound the observed mean fatigue life based on the observed mean obtained from a limited number of fatigue tests. The following describes how the confidence intervals were determined.

Assume that the overall fatigue life results are sampled from a normally distributed population with a mean $\mu$ and variance $\sigma^{2}$. Given $n$ samples from this population $\left(X_{1}+\right.$ $\left.\ldots+X_{n}\right)$, the sampled mean, $\bar{X}$, and variance, $S^{2}$, are defined as

$$
\begin{gathered}
\bar{X}=\left(X_{1}+\ldots+X_{n}\right) / n, \\
S^{2}=\frac{1}{n-1} \sum_{i=n}^{n}\left(X_{i}-\bar{X}\right)^{2} .
\end{gathered}
$$

It is readily shown that the quantity

$$
T=\frac{\bar{X}-\mu}{S / \sqrt{n}}
$$


has the probability density function

$$
f(t)=\frac{\Gamma((\nu+1) / 2)}{\sqrt{\nu \pi} \Gamma(\nu / 2)}\left(1+t^{2} / \nu\right)^{-(\nu+1) / 2}
$$

where $\nu$ is the umber of degrees of freedom and is equal to $n-1$ and $\Gamma$ is the Gamma function. This distribution is known as the Student's t-distribution, and is independent of the unobservable parameters $\mu$ and $\sigma^{2}$.

Using this probability distribution function, bounding intervals on the sampled mean and variance can be obtained. Suppose we would like to find the interval which would bound the sampled mean of a population $90 \%$ of the time for a sample population size $n$. Working with the parameter $T$, this is equivalent to selecting an number $A$ such that

$$
\operatorname{Pr}(-A<T<A)=0.9
$$

This is also equivalent to

$$
\operatorname{Pr}(T<A)=0.95, \operatorname{Pr}(T>-A)=0.95
$$

thus $\mathrm{A}$ is the 95 th percentile of this probability distribution, or $A=t_{0.5, n-1}$. More generally, for a confidence level of $(1-\alpha), A=t_{\alpha / 2, n-1}$. Subbing equation E.3 into equation E.5, we obtain

$$
\operatorname{Pr}\left(-A<\frac{\bar{X}-\mu}{S / \sqrt{n}}<A\right)=0.9,
$$

which can be rearranged to obtain

$$
\operatorname{Pr}\left(\bar{X}-A \frac{S}{\sqrt{n}}<\mu<\bar{X}+A \frac{S}{\sqrt{n}}\right)=0.9 .
$$

Therefore, the interval bound by

$$
\bar{X} \pm A \frac{S}{\sqrt{n}}
$$

is the $90 \%$ confidence interval for $\mu$. In more general terms, the $(1-\alpha)$ confidence interval 
is given by

$$
\bar{X} \pm \frac{t_{\alpha / 2, n-1} S}{\sqrt{n}}
$$

Using the above method, confidence intervals ranging from 50-90\% were determined for the countersunk and universal rivet fatigue specimens. The results of these calculations are summarized in Tables E.3 and E.4 and in Figure E.1.

Clearly, the effect of squeeze force on the population is different for the countersunk and universal rivet splices tested. Within a $90 \%$ confidence interval, each squeeze force represents a distinct population for the countersunk rivet splice configuration (Figure E.1). Conversely, the two lowest squeeze forces are nearly indistinguishable for the universal rivet joint configuration and there is a lower level of certainty for the distinctness of the populations at higher squeeze forces compared to its countersunk counterpart.

Table E.3: Confidence intervals for Type 1 fatigue specimens containing universal rivets (corrected fatigue life shown)

\begin{tabular}{|c|c|c|c|c|c|c|c|}
\hline $\begin{array}{c}F_{S q} \\
(\mathrm{lbf})\end{array}$ & $n$ & $\begin{array}{c}\bar{X} \\
\text { (kcycles) }\end{array}$ & $\begin{array}{c}S \\
\text { (kcycles) }\end{array}$ & Confidence Level & $t_{\alpha / 2, n-1}$ & $\begin{array}{l}\text { Upper Bound } \\
\text { (kcycles) }\end{array}$ & $\begin{array}{c}\text { Lower Bound } \\
\text { (kcycles) }\end{array}$ \\
\hline 1500 & 4 & 217.5 & 19.9 & $\begin{array}{l}0.5 \\
0.7 \\
0.8 \\
0.9\end{array}$ & $\begin{array}{l}0.765 \\
1.250 \\
1.638 \\
2.353\end{array}$ & $\begin{array}{l}209.9 \\
205.1 \\
201.2 \\
194.1\end{array}$ & $\begin{array}{l}225.1 \\
230.0 \\
233.8 \\
240.9\end{array}$ \\
\hline 2000 & 4 & 225.4 & 17.7 & $\begin{array}{l}0.5 \\
0.7 \\
0.8 \\
0.9\end{array}$ & $\begin{array}{l}0.765 \\
1.250 \\
1.638 \\
2.353\end{array}$ & $\begin{array}{l}218.6 \\
214.3 \\
210.8 \\
204.5\end{array}$ & $\begin{array}{l}232.1 \\
236.4 \\
239.9 \\
246.2\end{array}$ \\
\hline 2500 & 4 & 257.5 & 22.7 & $\begin{array}{l}0.5 \\
0.7 \\
0.8 \\
0.9\end{array}$ & $\begin{array}{l}0.765 \\
1.250 \\
1.638 \\
2.353\end{array}$ & $\begin{array}{l}248.8 \\
243.3 \\
238.9 \\
230.8\end{array}$ & $\begin{array}{l}266.2 \\
271.7 \\
276.1 \\
284.3\end{array}$ \\
\hline 3000 & 4 & 311.0 & 24.4 & $\begin{array}{l}0.5 \\
0.7 \\
0.8 \\
0.9\end{array}$ & $\begin{array}{l}0.765 \\
1.250 \\
1.638 \\
2.353\end{array}$ & $\begin{array}{l}301.6 \\
295.7 \\
291.0 \\
282.3\end{array}$ & $\begin{array}{l}320.3 \\
326.2 \\
330.9 \\
339.6\end{array}$ \\
\hline
\end{tabular}


Table E.4: Confidence intervals for Type 1 fatigue specimens containing countersunk rivets (corrected fatigue life shown)

\begin{tabular}{|c|c|c|c|c|c|c|c|}
\hline $\begin{array}{l}F_{S q} \\
(\mathrm{lbf})\end{array}$ & $n$ & $\begin{array}{c}\bar{X} \\
\text { (kcycles) }\end{array}$ & $\begin{array}{c}S \\
\text { (kcycles) }\end{array}$ & Confidence Level & $t_{\alpha / 2, n-1}$ & $\begin{array}{c}\text { Upper Bound } \\
\text { (kcycles) }\end{array}$ & $\begin{array}{c}\text { Lower Bound } \\
\text { (kcycles) }\end{array}$ \\
\hline 1500 & 6 & 117.5 & 7.7 & $\begin{array}{l}0.5 \\
0.7 \\
0.8 \\
0.9\end{array}$ & $\begin{array}{l}0.727 \\
1.156 \\
1.476 \\
2.015\end{array}$ & $\begin{array}{l}115.3 \\
113.9 \\
112.9 \\
111.2\end{array}$ & $\begin{array}{l}119.8 \\
121.2 \\
122.2 \\
123.8\end{array}$ \\
\hline 2000 & 6 & 149.9 & 18.5 & $\begin{array}{l}0.5 \\
0.7 \\
0.8 \\
0.9\end{array}$ & $\begin{array}{l}0.727 \\
1.156 \\
1.476 \\
2.015\end{array}$ & $\begin{array}{l}144.5 \\
141.2 \\
138.8 \\
134.7\end{array}$ & $\begin{array}{l}155.4 \\
158.7 \\
161.1 \\
165.1\end{array}$ \\
\hline 2500 & 10 & 196.9 & 49.2 & $\begin{array}{l}0.5 \\
0.7 \\
0.8 \\
0.9\end{array}$ & $\begin{array}{l}0.703 \\
1.100 \\
1.383 \\
1.833\end{array}$ & $\begin{array}{l}185.9 \\
179.8 \\
175.4 \\
168.3\end{array}$ & $\begin{array}{l}207.8 \\
214.0 \\
218.4 \\
225.4\end{array}$ \\
\hline 3000 & 9 & 349.4 & 63.3 & $\begin{array}{l}0.5 \\
0.7 \\
0.8 \\
0.9\end{array}$ & $\begin{array}{l}0.706 \\
1.108 \\
1.397 \\
1.860\end{array}$ & $\begin{array}{l}334.5 \\
326.0 \\
319.9 \\
310.1\end{array}$ & $\begin{array}{l}364.3 \\
372.8 \\
378.9 \\
388.7\end{array}$ \\
\hline
\end{tabular}

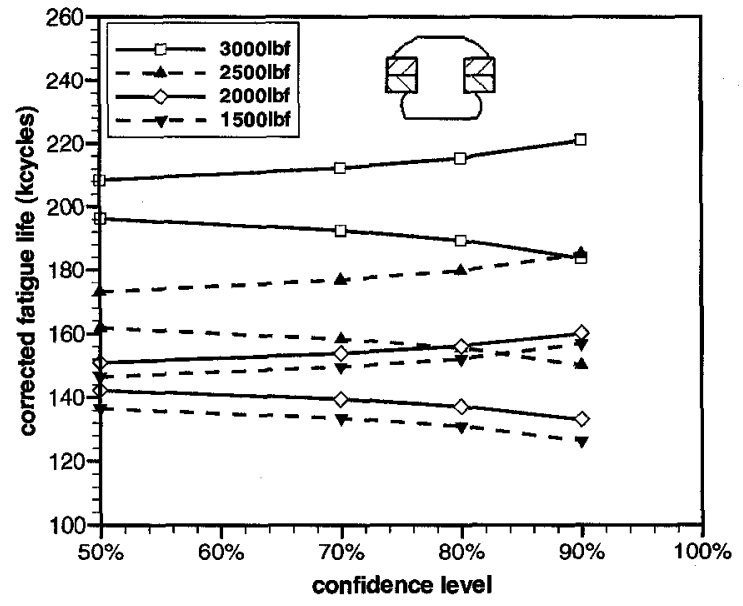

(a) universal rivet

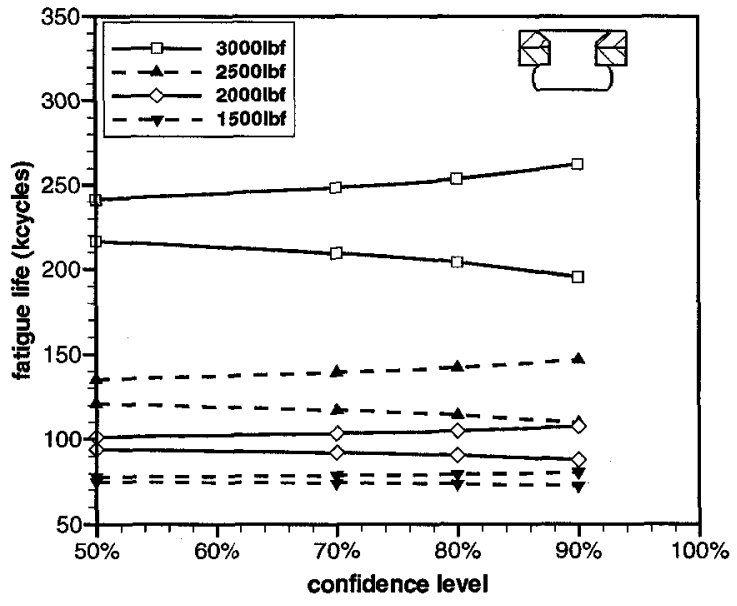

(b) countersunk rivet

Figure E.1: Mean confidence intervals for outer sheet failure fatigue life 


\section{Appendix F}

\section{SEM Crack Growth Reconstruction Data}

The following appendix contains all of the SEM crack front location results described in Chapter 6. All results are plotted in a local coordinate system where the origin corresponds to the hole edge as observed on the fracture surface. Crack lengths, defined in Figure F.1, are measured relative to this origin. Figure F.2 shows the location of each of the cracks from the set of reconstructions to provide an indication of the relative position of the local origin in each plot.

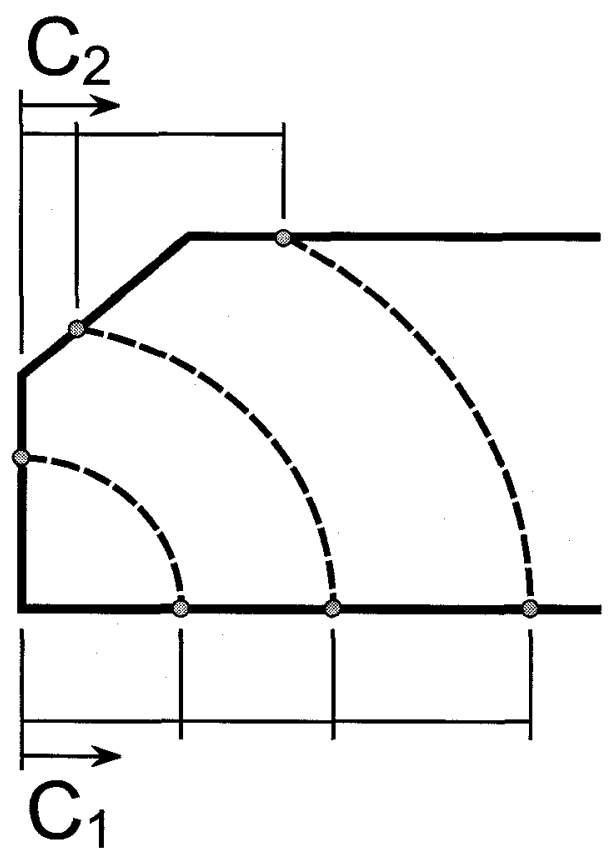

Figure F.1: Crack length definition 

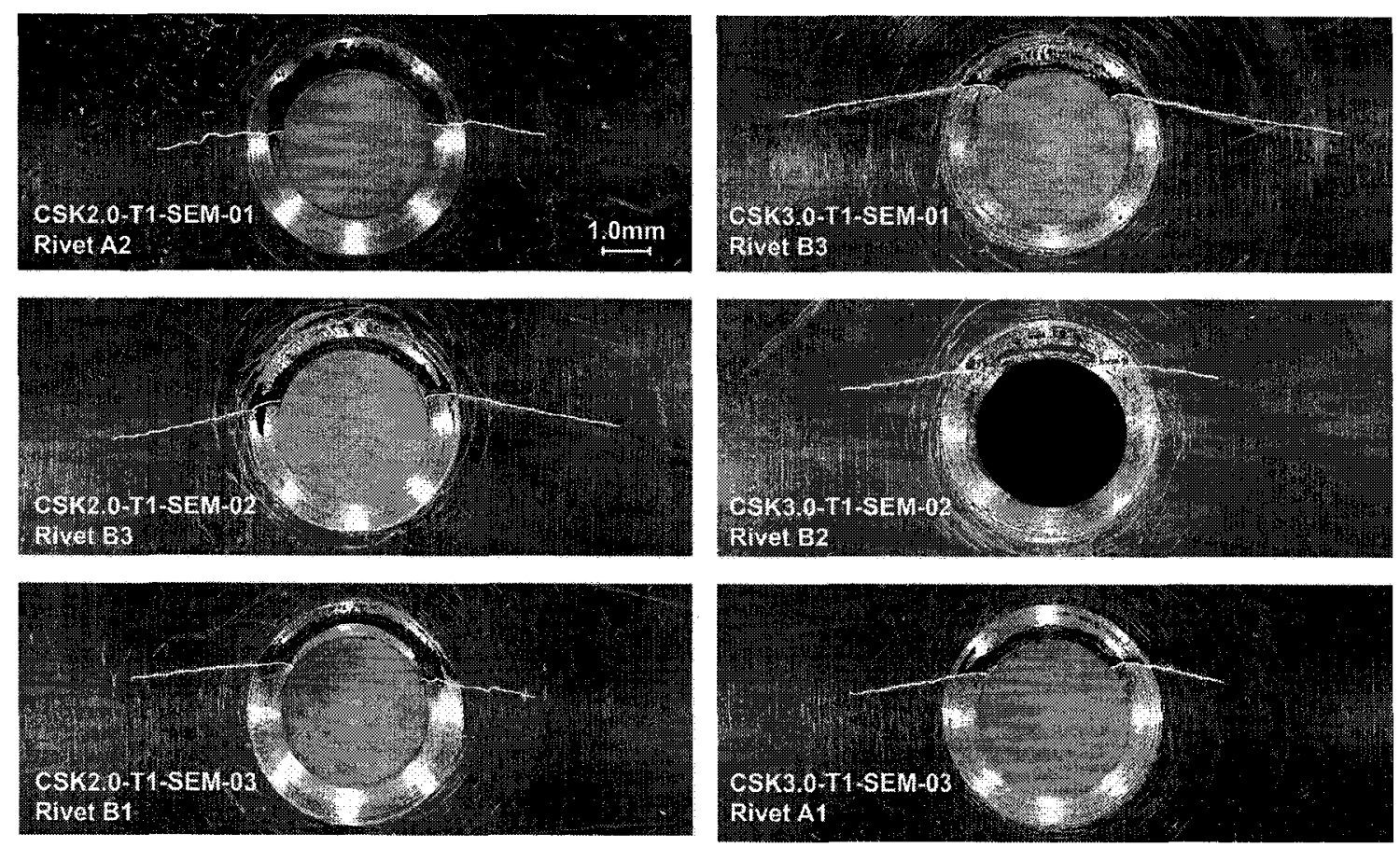

Figure F.2: Location and size of cracks prior to SEM reconstruction 


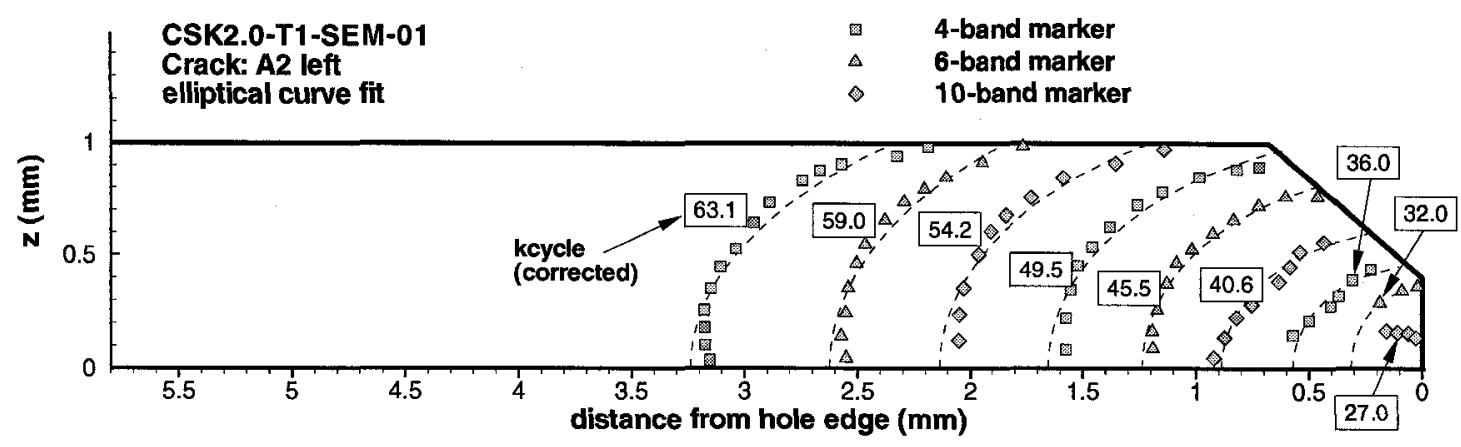

Figure F.3: Crack front plot for CSK2.0-T1-SEM-01 rivet A2 left side

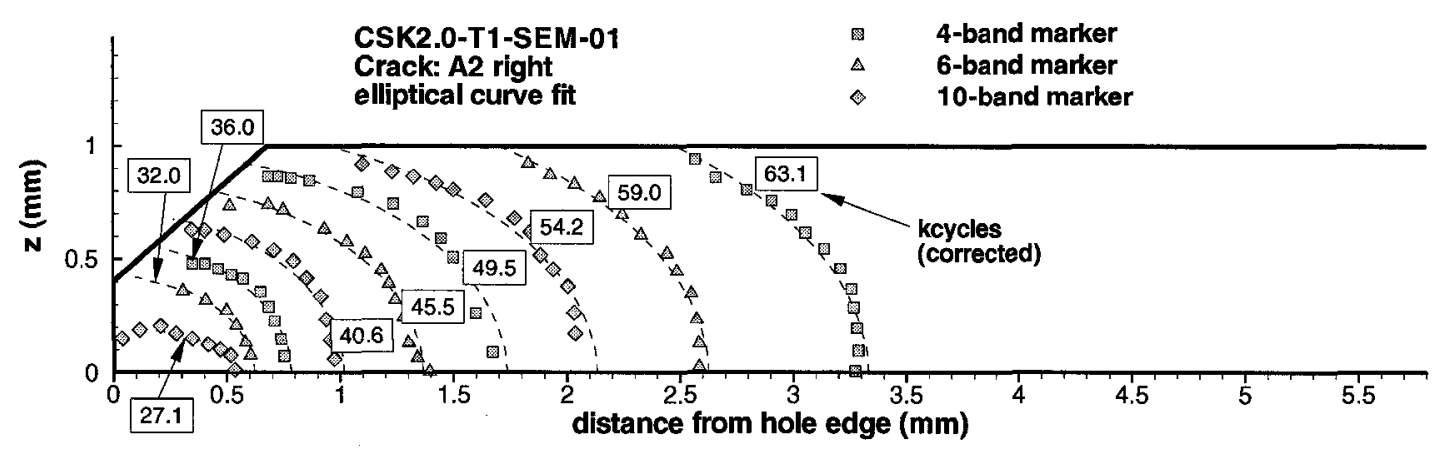

Figure F.4: Crack front plot for CSK2.0-T1-SEM-01 rivet A2 right side

Table F.1: Crack growth reconstruction data for CSK2.0-T1-SEM-01 rivet A2

\begin{tabular}{cccccccc}
\hline \multicolumn{2}{c}{ Specimen: CSK2.0-T1-SEM-01 } & \multicolumn{7}{c}{ Rivet Number: A2 } \\
\hline \multicolumn{3}{c}{ left } \\
(cycles) & $\mathrm{a}(\mathrm{mm})$ & $\mathrm{c}_{1}(\mathrm{~mm})$ & $\mathrm{c}_{2}(\mathrm{~mm})$ & $\mathrm{N}$ (cycles) & $\mathrm{a}(\mathrm{mm})$ & $\mathrm{c}_{1}(\mathrm{~mm})$ & $\mathrm{c}_{2}(\mathrm{~mm})$ \\
\hline 63082 & 1.447 & 3.239 & 2.340 & 63082 & 1.500 & 3.332 & 2.483 \\
59047 & 1.404 & 2.625 & 1.842 & 59047 & 1.312 & 2.626 & 1.700 \\
54152 & 1.210 & 2.137 & 1.204 & 54152 & 1.117 & 2.133 & 0.950 \\
49544 & 1.046 & 1.654 & 0.658 & 49544 & 0.984 & 1.735 & 0.451 \\
45509 & 0.871 & 1.238 & 0.488 & 45509 & 0.847 & 1.365 & 0.446 \\
40614 & 0.622 & 0.887 & 0.278 & 40614 & 0.706 & 1.017 & 0.398 \\
36006 & 0.458 & 0.572 & 0.138 & 36006 & 0.569 & 0.784 & 0.203 \\
31971 & 0.363 & 0.315 & 0.048 & 31971 & 0.426 & 0.622 & 0.101 \\
& & & & 27076 & 0.197 & 0.551 & 0.000 \\
\hline
\end{tabular}




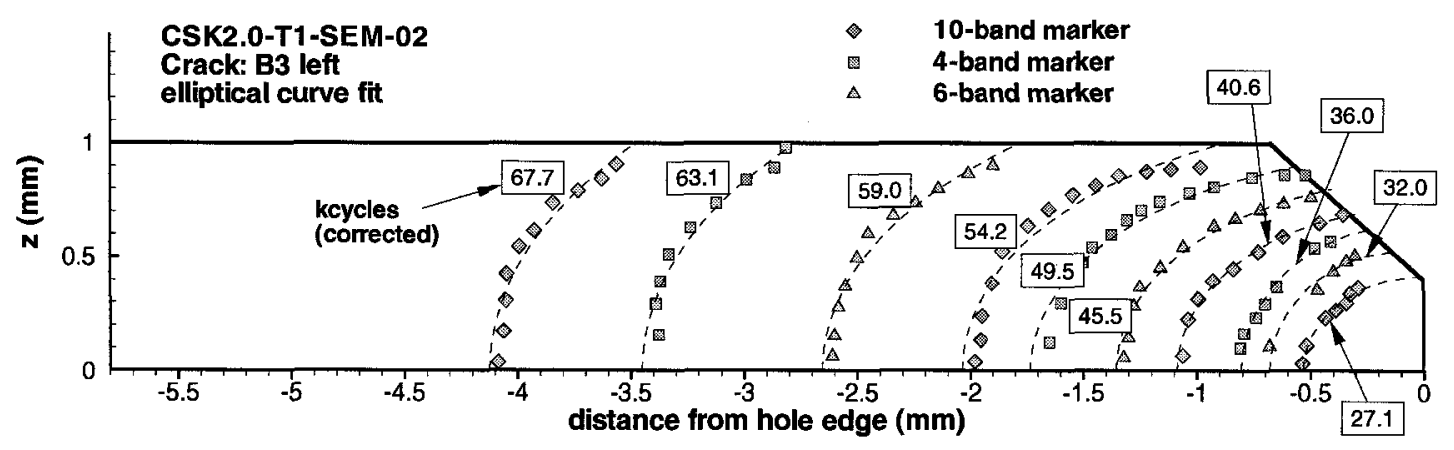

Figure F.5: Crack front plot for CSK2.0-T1-SEM-02 rivet B3 left side

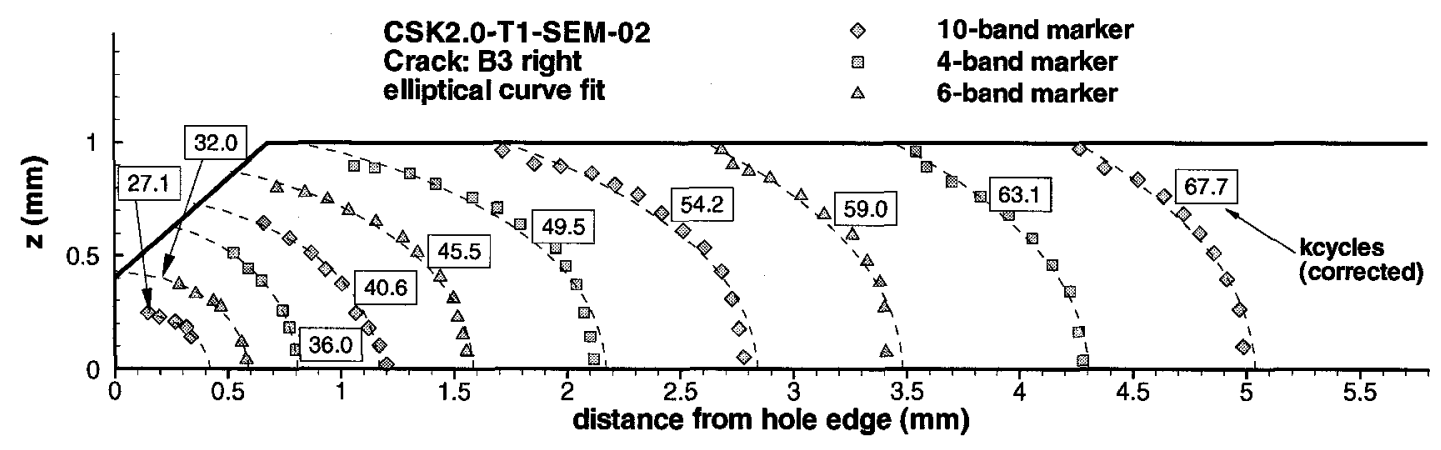

Figure F.6: Crack front plot for CSK2.0-T1-SEM-02 rivet B3 right side

Table F.2: Crack growth reconstruction data for CSK2.0-T1-SEM-02 rivet B3

\begin{tabular}{cccccccc}
\hline \multicolumn{7}{c}{ Specimen: CSK2.0-T1-SEM-02 } & \multicolumn{5}{c}{ Rivet Number: B3 } \\
\hline & \multicolumn{3}{c}{ left } \\
$\mathrm{N}$ (cycles) & $\mathrm{a}(\mathrm{mm})$ & $\mathrm{c}_{1}(\mathrm{~mm})$ & $\mathrm{c}_{2}(\mathrm{~mm})$ & $\mathrm{N}($ cycles $)$ & $\mathrm{a}(\mathrm{mm})$ & $\mathrm{c}_{1}(\mathrm{~mm})$ & $\mathrm{c}_{2}(\mathrm{~mm})$ \\
\hline 67690 & 1.863 & 4.124 & 3.480 & 67690 & 1.872 & 5.038 & 4.259 \\
63082 & 1.680 & 3.451 & 2.773 & 63082 & 1.665 & 4.304 & 3.441 \\
59047 & 1.349 & 2.656 & $\mathbf{1 . 7 8 2}$ & 59047 & 1.513 & 3.481 & 2.612 \\
54152 & 1.110 & 2.038 & 0.886 & 54152 & 1.256 & 2.840 & 1.719 \\
49544 & 0.953 & 1.735 & 0.580 & 49544 & 1.077 & 2.171 & 0.804 \\
45509 & 0.838 & 1.356 & 0.466 & 45509 & 0.926 & 1.585 & 0.549 \\
40614 & 0.721 & 1.088 & 0.358 & 40614 & 0.770 & 1.167 & $\mathbf{0 . 4 0 2}$ \\
36006 & 0.657 & 0.806 & 0.293 & 36006 & 0.668 & 0.807 & 0.300 \\
31971 & 0.549 & 0.681 & 0.209 & 31971 & 0.430 & 0.590 & 0.105 \\
27076 & 0.419 & 0.532 & $\mathbf{0 . 0 9 8}$ & 27076 & 0.267 & 0.420 & 0.000 \\
\hline
\end{tabular}




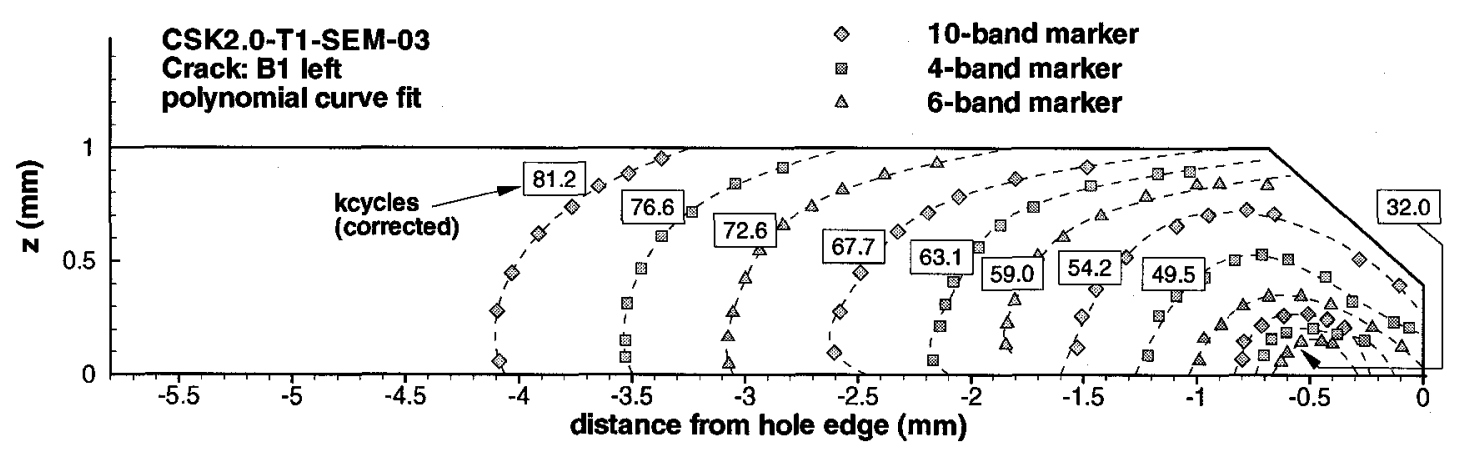

Figure F.7: Crack front plot for CSK2.0-T1-SEM-03 rivet B1 left side

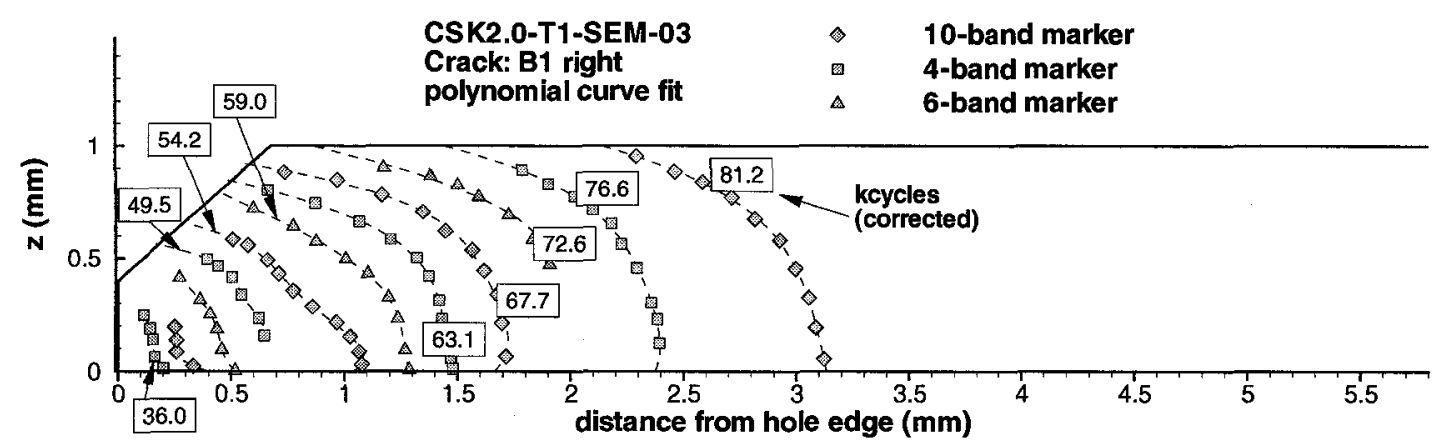

Figure F.8: Crack front plot for CSK2.0-T1-SEM-03 rivet B1 right side

Table F.3: Crack growth reconstruction data for CSK2.0-T1-SEM-03 rivet B1

\begin{tabular}{cccccc}
\hline \multicolumn{2}{c}{ Specimen: CSK2.0-T1-SEM-03 } & \multicolumn{4}{c}{ Rivet Number: B1 } \\
\hline $\begin{array}{c}\text { left } \\
\mathrm{N} \text { (cycles) }\end{array}$ & $\mathrm{c}_{1}(\mathrm{~mm})$ & $\mathrm{c}_{2}(\mathrm{~mm})$ & $\mathrm{N}$ (cycles) & $\begin{array}{c}\text { right } \\
\mathrm{c}_{1}(\mathrm{~mm})\end{array}$ & $\mathrm{c}_{2}(\mathrm{~mm})$ \\
\hline 81228 & 4.060 & 3.240 & 81228 & 3.130 & 2.140 \\
76620 & 3.520 & 2.550 & 76620 & 2.390 & 1.440 \\
72585 & 3.060 & 1.820 & 72585 & 1.710 & 0.860 \\
67690 & 2.600 & 0.890 & 67690 & 1.480 & 0.580 \\
63082 & 2.160 & & 63082 & 1.290 & 0.500 \\
59047 & 1.840 & & 59047 & 1.080 & 0.450 \\
54152 & 1.530 & & 49544 & 0.520 & \\
49544 & 1.220 & & 45509 & 0.330 & \\
4509 & 0.990 & & 40614 & 0.200 & \\
40614 & 0.800 & & & & \\
36006 & 0.710 & & & & \\
31971 & 0.630 & & & &
\end{tabular}




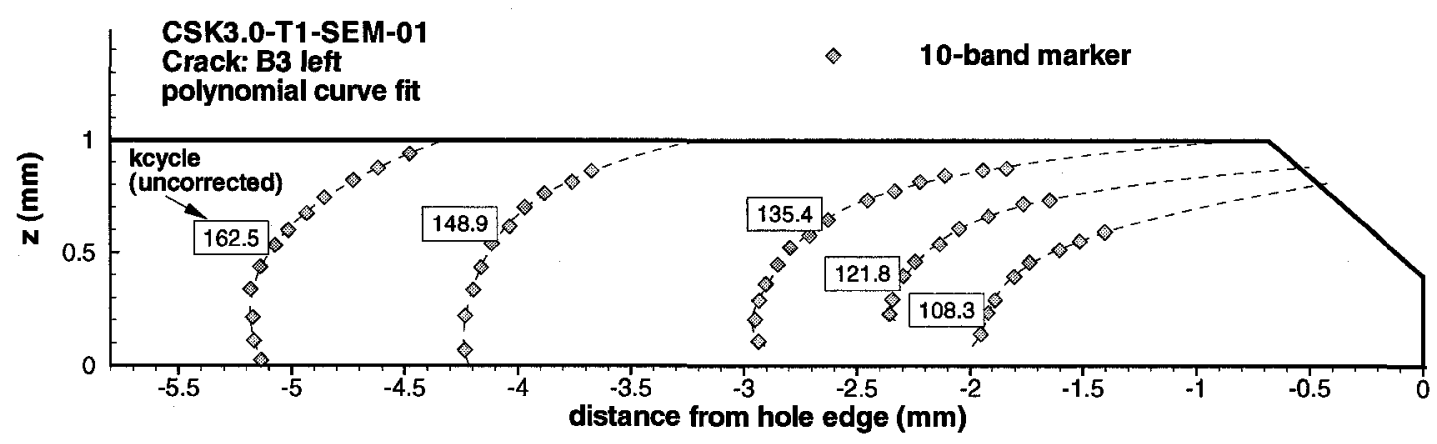

Figure F.9: Crack front plot for CSK3.0-T1-SEM-01 rivet B3 left side

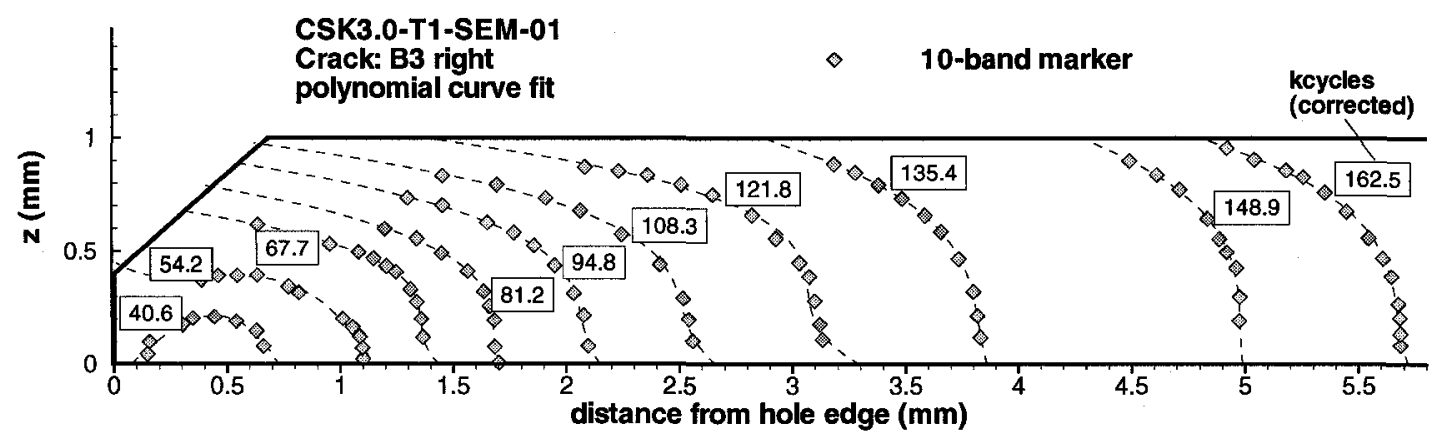

Figure F.10: Crack front plot for CSK3.0-T1-SEM-01 rivet B3 right side

Table F.4: Crack growth reconstruction data for CSK3.0-T1-SEM-01 rivet B3

\begin{tabular}{cccccc}
\hline \multicolumn{3}{c}{ Specimen: CSK3.0-T1-SEM-01 } & \multicolumn{4}{c}{ Rivet Number: B3 } \\
\hline $\mathrm{N}$ (cycles) & $\mathrm{c}_{1}(\mathrm{~mm})$ & $\mathrm{c}_{2}(\mathrm{~mm})$ & $\mathrm{N}$ (cycles) & $\mathrm{c}_{1}(\mathrm{~mm})$ & $\mathrm{c}_{2}(\mathrm{~mm})$ \\
\hline 162456 & 5.122 & 4.320 & 162456 & 5.719 & 4.818 \\
158421 & 4.992 & 4.106 & 153526 & 5.344 & 4.617 \\
153526 & 4.766 & 3.803 & 148918 & 4.989 & 4.291 \\
148918 & 4.226 & 3.203 & 139988 & 4.112 & 3.308 \\
144883 & 3.614 & & 135380 & 3.859 & 2.878 \\
139988 & 3.263 & & 126450 & 3.361 & \\
135380 & 2.892 & & 121842 & 3.142 & \\
131345 & 2.747 & & 112912 & 2.781 & \\
126450 & 2.494 & & 108304 & 2.579 & \\
121842 & 2.286 & & 99374 & 2.307 & \\
117807 & 2.198 & & 94766 & 2.113 & \\
112912 & 2.118 & & 85836 & 1.843 & \\
108304 & 1.957 & & 81228 & 1.701 & \\
104269 & 1.810 & & 72298 & 1.484 & \\
& & 67690 & 1.379 & \\
& & 58760 & 1.224 & \\
& & 54152 & 1.104 & \\
& & & 45222 & 0.879 & \\
\hline
\end{tabular}




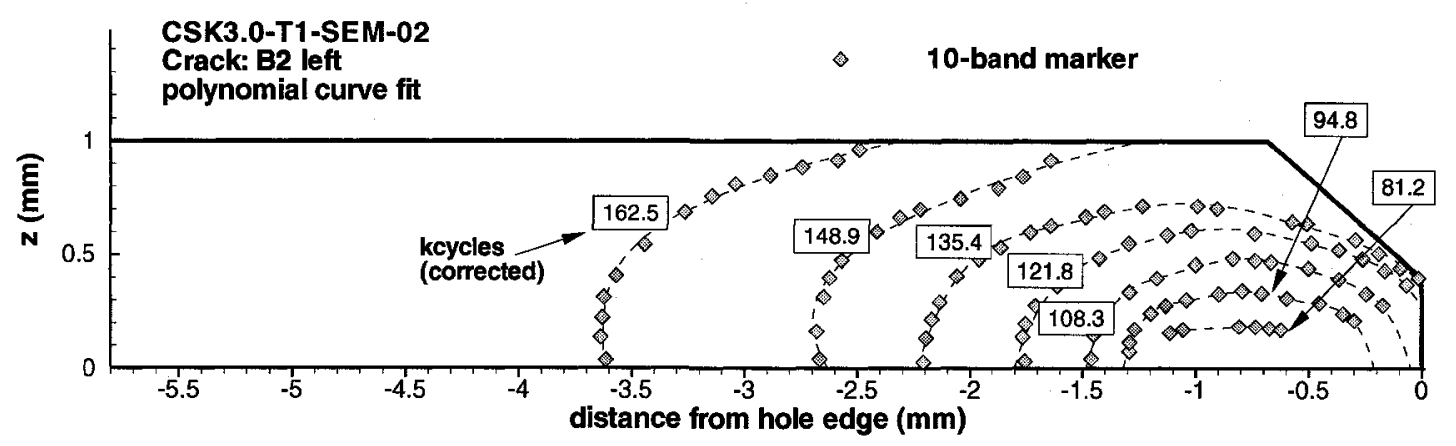

Figure F.11: Crack front plot for CSK3.0-T1-SEM-02 rivet B2 left side

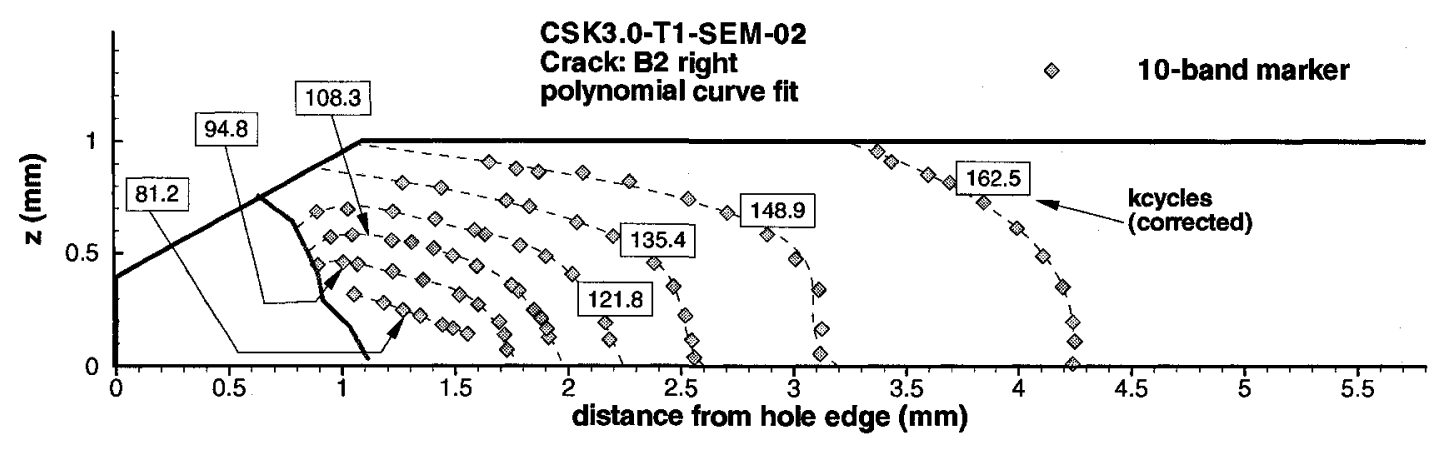

Figure F.12: Crack front plot for CSK3.0-T1-SEM-02 rivet B2 right side

Table F.5: Crack growth reconstruction data for CSK3.0-T1-SEM-02 rivet B2

\begin{tabular}{cccccc}
\hline \multicolumn{2}{c}{ Specimen: } & CSK3.0-T1-SEM-02 & \multicolumn{4}{c}{ Rivet Number: B2 } \\
\hline \multirow{2}{*}{$\mathrm{N}$ (cycles) } & $\mathrm{c}_{1}(\mathrm{~mm})$ & $\mathrm{c}_{2}(\mathrm{~mm})$ & $\mathrm{N}$ (cycles) & $\mathrm{c}_{1}(\mathrm{~mm})$ & $\mathrm{c}_{2}(\mathrm{~mm})$ \\
\hline 162456 & 3.612 & 2.325 & 162456 & 4.239 & 3.244 \\
148918 & 2.666 & 1.251 & 157848 & 3.813 & 2.388 \\
135380 & 2.206 & & 153813 & 3.453 & 1.649 \\
121842 & 1.752 & & 148918 & 3.119 & 1.086 \\
108304 & 1.458 & & 144310 & 2.925 & 1.036 \\
94766 & 1.29 & & 140275 & 2.742 & 0.984 \\
& & 135380 & 2.557 & 0.871 \\
& & & 130772 & 2.42 & 0.819 \\
& & 126737 & 2.304 & 0.804 \\
& & 121842 & 2.237 & \\
& & & 108304 & 1.973 & \\
\hline
\end{tabular}




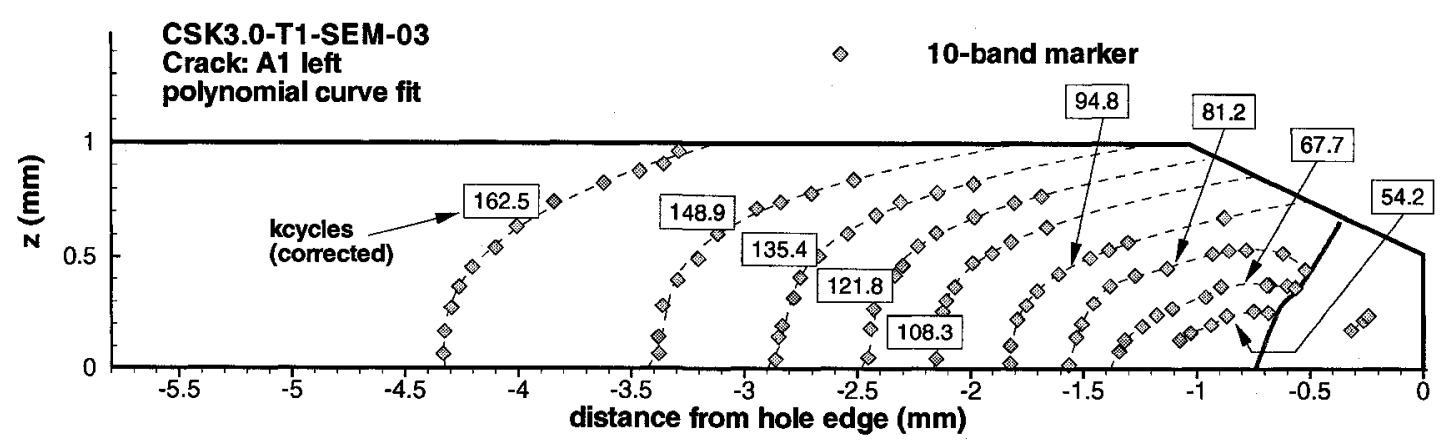

Figure F.13: Crack front plot for CSK3.0-T1-SEM-03 rivet A1 left side

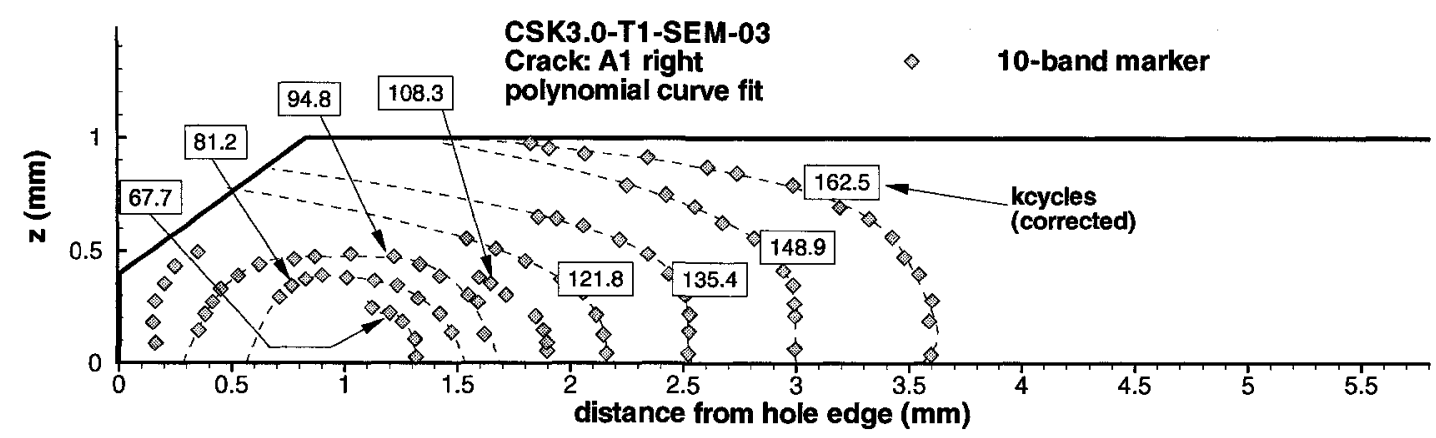

Figure F.14: Crack front plot for CSK3.0-T1-SEM-03 rivet A1 right side

Table F.6: Crack growth reconstruction data for CSK3.0-T1-SEM-03 rivet A1

\begin{tabular}{cccccc}
\hline \multicolumn{3}{c}{ Specimen: CSK3.0-T1-SEM-03 } & \multicolumn{4}{c}{ Rivet Number: A1 } \\
\hline $\mathrm{N}$ (cycles) & $\mathrm{c}_{1}(\mathrm{~mm})$ & $\mathrm{c}_{2}(\mathrm{~mm})$ & $\mathrm{N}$ (cycles) & $\mathrm{c}_{1}(\mathrm{~mm})$ & $\mathrm{c}_{2}(\mathrm{~mm})$ \\
\hline 162456 & 4.330 & 3.282 & 162456 & 3.596 & 1.817 \\
157848 & 3.944 & 2.693 & 148918 & 2.994 & \\
153813 & 3.615 & & 135380 & 2.521 & \\
148918 & 3.378 & & 121842 & 2.160 & \\
135380 & 2.862 & & 108304 & 1.898 & \\
121842 & 2.454 & & 94766 & 1.630 & \\
108304 & 2.154 & & 67690 & 1.319 & \\
94766 & 1.823 & & & & \\
81228 & 1.563 & & & & \\
67690 & 1.376 & & &
\end{tabular}




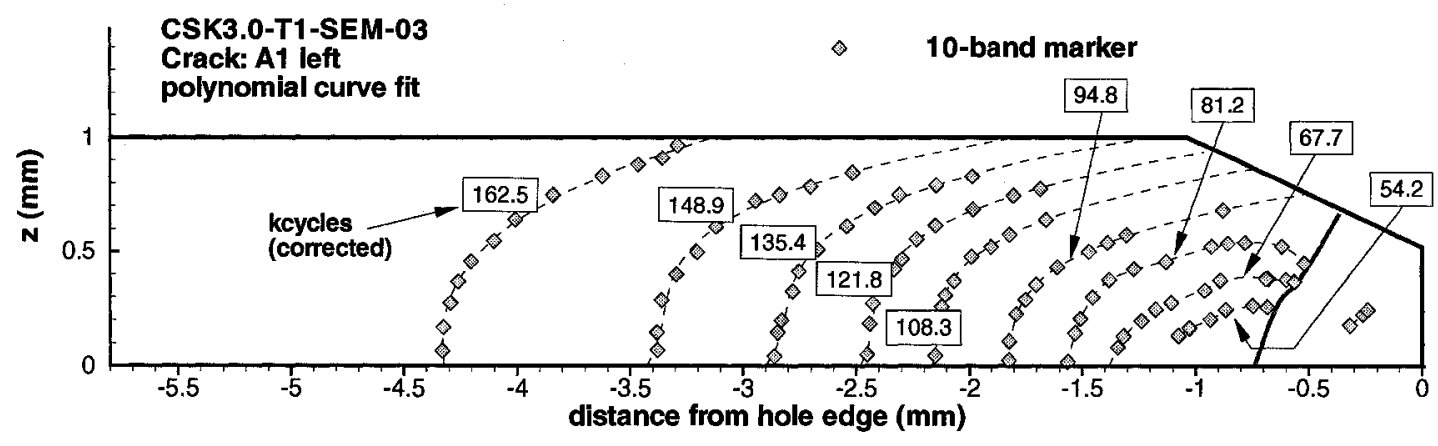

Figure F.13: Crack front plot for CSK3.0-T1-SEM-03 rivet A1 left side

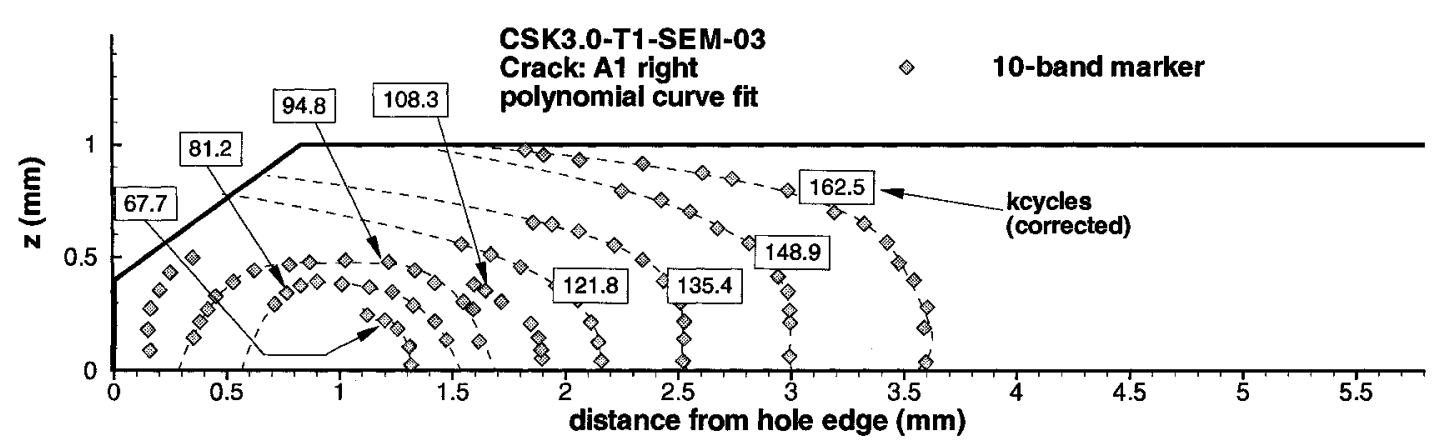

Figure F.14: Crack front plot for CSK3.0-T1-SEM-03 rivet A1 right side

Table F.6: Crack growth reconstruction data for CSK3.0-T1-SEM-03 rivet A1

\begin{tabular}{|c|c|c|c|c|c|}
\hline \multicolumn{3}{|c|}{ Specimen: CSK3.0-T1-SEM-03 } & \multicolumn{2}{|c|}{ Rivet Number: $\mathbf{A} 1$} & \\
\hline $\mathrm{N}$ (cycles) & $\begin{array}{c}\text { left } \\
c_{1}(\mathrm{~mm})\end{array}$ & $\mathrm{c}_{2}(\mathrm{~mm})$ & $\mathrm{N}$ (cycles) & $\begin{array}{c}\text { right } \\
c_{1}(\mathrm{~mm})\end{array}$ & $\mathrm{c}_{2}(\mathrm{~mm})$ \\
\hline $\begin{array}{c}162456 \\
157848 \\
153813 \\
148918 \\
135380 \\
121842 \\
108304 \\
94766 \\
81228 \\
67690\end{array}$ & $\begin{array}{l}4.330 \\
3.944 \\
3.615 \\
3.378 \\
2.862 \\
2.454 \\
2.154 \\
1.823 \\
1.563 \\
1.376\end{array}$ & $\begin{array}{l}3.282 \\
2.693\end{array}$ & $\begin{array}{c}162456 \\
148918 \\
135380 \\
121842 \\
108304 \\
94766 \\
67690\end{array}$ & $\begin{array}{l}3.596 \\
2.994 \\
2.521 \\
2.160 \\
1.898 \\
1.630 \\
1.319\end{array}$ & 1.817 \\
\hline
\end{tabular}

